\title{
Enantioselective Synthesis of Indole-Annulated Medium-Sized Rings
}

\author{
Lin Huang ${ }^{\dagger}$, Li-Xin Dai ${ }^{\dagger}$ and Shu-Li You ${ }^{* \dagger}$ \\ 'State Key Laboratory of Organometallic Chemistry, Shanghai Institute of Organic \\ Chemistry, Chinese Academy of Sciences, 345 Lingling Lu, Shanghai 200032, China \\ * Collaborative Innovation Center of Chemical Science and Engineering (Tianjin) \\ E-mail: $\underline{\text { slyou@ sioc.ac.cn }}$
}

\section{Table of Contents}

General methods

S2

Table S1. Optimization of Conditions with Lewis Acids

S3

Experimental details and characterization data

S4-S39

Copies of NMR spectra and HPLC chromatographs

S40-S127 
General methods. Unless stated otherwise, all reactions were carried out in flame-dried glassware under a dry argon atmosphere. All solvents were purified and dried according to standard methods prior to use.

${ }^{1} \mathrm{H}$ and ${ }^{13} \mathrm{C}$ NMR spectra were recorded on a Varian instrument $(400 \mathrm{MHz}$ and 101 $\mathrm{Hz}$ respectively) or an Agilent instrument $(400,600 \mathrm{MHz}$ and 101, $151 \mathrm{MHz}$, respectively) and internally referenced to tetramethylsilane signal or residual protic solvent signals. ${ }^{19} \mathrm{~F}$ NMR spectra were recorded on a Varian instrument $(376 \mathrm{MHz}$, respectively) and referenced relative to $\mathrm{CFCl}_{3}$. Data for ${ }^{1} \mathrm{H}$ NMR are recorded as follows: chemical shift $(\delta, \mathrm{ppm})$, multiplicity $(\mathrm{s}=$ singlet, $\mathrm{d}=$ doublet, $\mathrm{t}=$ triplet, $\mathrm{m}=$ multiplet or unresolved, br = broad singlet, coupling constant(s) in Hz, integration). Data for ${ }^{13} \mathrm{C}$ NMR are reported in terms of chemical shift $(\delta, \mathrm{ppm})$. 
Table S1. Optimization of Conditions with Lewis Acids ${ }^{a}$
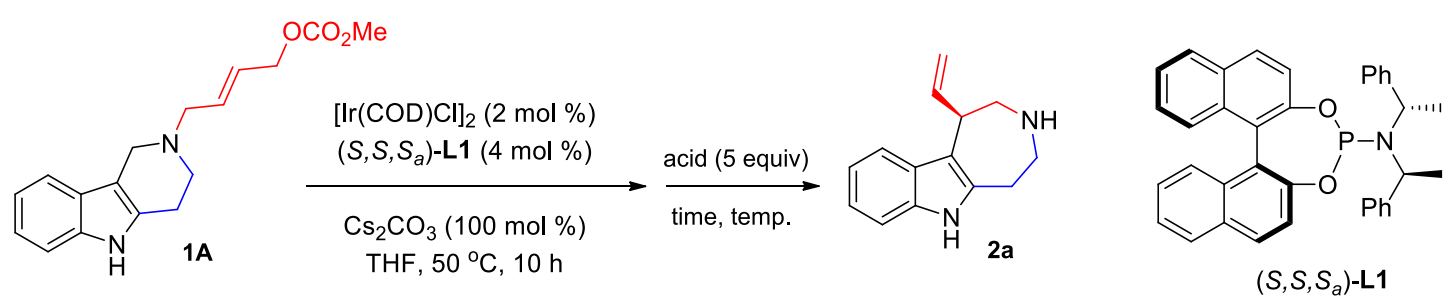

\begin{tabular}{cccccc}
\hline entry & acid & temp. $\left({ }^{\circ} \mathrm{C}\right)$ & time $(\mathrm{h})$ & yield $(\%)^{b}$ & ee $(\%)^{c}$ \\
\hline 1 & --- & $\mathrm{rt}$ & 16 & 39 & 95 \\
2 & $\mathrm{AcOH}$ & $\mathrm{rt}$ & 16 & 43 & 92 \\
3 & $\mathrm{CF}_{3} \mathrm{COOH}$ & $\mathrm{rt}$ & 16 & 40 & 88 \\
4 & ${\mathrm{TsOH} \cdot \mathrm{H}_{2} \mathrm{O}}_{5}$ & $\mathrm{rt}$ & 16 & 31 & 87 \\
6 & $\mathrm{BF}_{3} \cdot \mathrm{OEt}_{2}$ & $\mathrm{rt}$ & 16 & --- & -- \\
\hline
\end{tabular}

${ }^{a}$ Reaction conditions: $2 \mathrm{~mol} \%$ of $[\operatorname{Ir}(\mathrm{COD}) \mathrm{Cl}]_{2}, 4 \mathrm{~mol} \%$ of ligand $\mathbf{L 1}, 0.4 \mathrm{mmol}$ of $1 \mathrm{~A}$ and $0.4 \mathrm{mmol}$ of $\mathrm{Cs}_{2} \mathrm{CO}_{3}$ in THF $(4.0 \mathrm{~mL})$. Catalyst was prepared by $n-\mathrm{PrNH}_{2}$ activation. ${ }^{b}$ Isolated yield. ${ }^{c}$ Determined by HPLC analysis. 


\section{General Procedure for the Synthesis of Substituted Allylic Carbonates of Indole}

\section{Derivatives:}

\section{1) Synthesis of Substrate $1 \mathrm{~A}$}

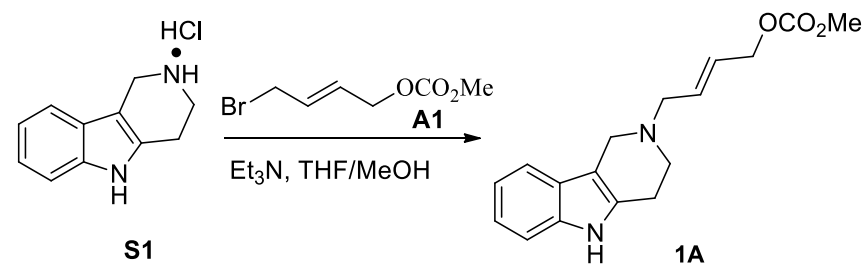

Tetrahydro- $\gamma$-carboline monohydrochloride $\mathbf{S 1}$ was synthesized according to Lit. ${ }^{1}$

(E)-4-Bromobut-2-en-1-yl methyl carbonate (A1, $0.63 \mathrm{~g}, 3 \mathrm{mmol}, 1.5$ equiv) was added to a solution of $\mathbf{S 1}\left(0.42 \mathrm{~g}, 2 \mathrm{mmol}, 1.0\right.$ equiv) and $\mathrm{Et}_{3} \mathrm{~N}(1 \mathrm{~mL}, 6 \mathrm{mmol}, 3$ equiv) in dry THF/MeOH $(\mathrm{v} / \mathrm{v}=5 / 1,25 \mathrm{~mL})$ at $0{ }^{\circ} \mathrm{C}$. The ice bath was removed and the reaction mixture was stirred at $\mathrm{rt}$ until the starting material was consumed completely (monitored by TLC). The crude reaction mixture was quenched with water and extracted with EtOAc $(50 \mathrm{~mL} \times 3)$. The organic layer was washed with brine, dried over $\mathrm{Na}_{2} \mathrm{SO}_{4}$, filtered and concentrated by rotary evaporation. Then the residue was purified by $\mathrm{Al}_{2} \mathrm{O}_{3}$ column chromatography $(\mathrm{PE} / \mathrm{EA}=4 / 1,2 / 1)$ to afford the desired product 1A. Viscous yellow oil. ${ }^{1} \mathrm{H}$ NMR $\left(400 \mathrm{MHz}, \mathrm{CDCl}_{3}\right) \delta 7.89(\mathrm{~s}, 1 \mathrm{H})$, $7.40(\mathrm{~d}, J=7.6 \mathrm{~Hz}, 1 \mathrm{H}), 7.27(\mathrm{~d}, J=8.0 \mathrm{~Hz}, 2 \mathrm{H}), 7.15-7.02(\mathrm{~m}, 2 \mathrm{H}), 6.06-5.93(\mathrm{~m}$, 1H), 5.90-5.81 (m, 1H), $4.67(\mathrm{~d}, J=6.0 \mathrm{~Hz}, 2 \mathrm{H}), 3.80(\mathrm{~s}, 3 \mathrm{H}), 3.71(\mathrm{~s}, 2 \mathrm{H}), 3.30$ (d, $J$ $=6.4 \mathrm{~Hz}, 2 \mathrm{H}), 2.94-2.79(\mathrm{~m}, 4 \mathrm{H}) .{ }^{13} \mathrm{C} \mathrm{NMR}\left(101 \mathrm{MHz}, \mathrm{CDCl}_{3}\right) \delta 155.6,136.1,132.5$, $132.0,126.9,126.0,121.1,119.1,117.4,110.7,108.0,67.9,59.4,54.8,50.2,49.6$, 23.4. IR (thin film): $v_{\max }\left(\mathrm{cm}^{-1}\right)=3407,3367,3059,2952,2914,2823,2764,1748$, 1449, 1359, 1272, 1097, 978, 949, 899, 790, 738. HRMS-ESI calcd for $\mathrm{C}_{17} \mathrm{H}_{21} \mathrm{~N}_{2} \mathrm{O}_{3}$ $[\mathrm{M}+\mathrm{H}]^{+}:$301.1547, Found: 301.1543 


\section{2) Synthesis of Substrates 1a-1i}

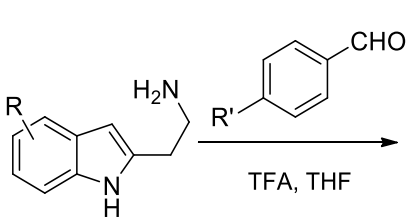

S2
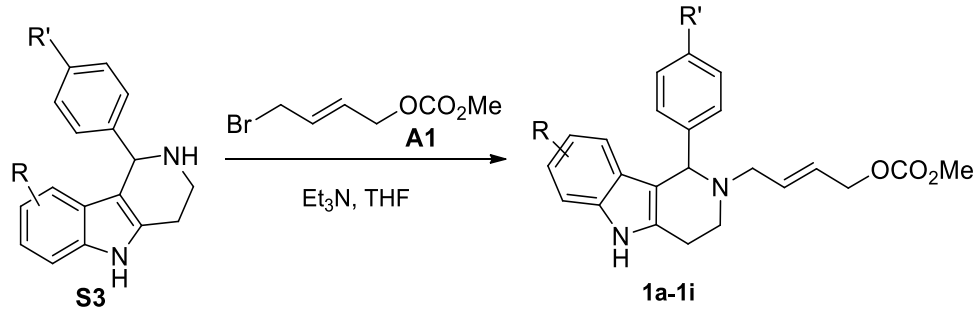

2-(2-Aminoethyl)indole derivatives $\mathbf{S 2}$ were obtained according to Lit. $^{2}$

Trifluoroacetic acid ( $0.22 \mathrm{~mL}, 3 \mathrm{mmol}, 1.5$ equiv) was added to a solution of the 2-(2-aminoethyl)indole derivative (S2, $2 \mathrm{mmol}, 1$ equiv) and substituted benzaldehyde $(0.41 \mathrm{ml}, 4 \mathrm{mmol}, 2$ equiv) in THF $(20 \mathrm{~mL})$. The reaction mixture was stirred for $5 \mathrm{~h}$ at $\mathrm{rt}$, then quenched with a $10 \% \mathrm{~K}_{2} \mathrm{CO}_{3}$ aqueous solution $(30 \mathrm{~mL})$ and extracted with $\mathrm{CH}_{2} \mathrm{Cl}_{2}$. The organic layer was dried over $\mathrm{Na}_{2} \mathrm{SO}_{4}$, filtered and concentrated by rotary evaporation. Then the residue was purified by $\mathrm{Al}_{2} \mathrm{O}_{3}$ column chromatography $(\mathrm{DCM} / \mathrm{MeOH}=50 / 1,20 / 1)$ to afford the crude product $\mathbf{S 3}$.

(E)-4-Bromobut-2-en-1-yl methyl carbonate (A1, $0.63 \mathrm{~g}, 3 \mathrm{mmol}, 1.5$ equiv) was added to a solution of $\mathbf{S 3}$ ( $2 \mathrm{mmol}, 1.0$ equiv) and $\mathrm{Et}_{3} \mathrm{~N}$ ( $1 \mathrm{~mL}, 6 \mathrm{mmol}, 3$ equiv) in THF $(25 \mathrm{~mL})$ at $0{ }^{\circ} \mathrm{C}$. Then the ice bath was removed and the reaction mixture was stirred at rt until the starting material was consumed completely (monitored by TLC). The crude reaction mixture was quenched with water and extracted with EtOAc (20 $\mathrm{mL} \times 3$ ). The organic layer was washed with brine, dried over $\mathrm{Na}_{2} \mathrm{SO}_{4}$, filtered and concentrated by rotary evaporation. The residue was purified by $\mathrm{Al}_{2} \mathrm{O}_{3}$ column chromatography $(\mathrm{PE} / \mathrm{EA}=4 / 1,2 / 1)$ to afford the desired product 1a-1i. The analytical data of the products are summarized below.

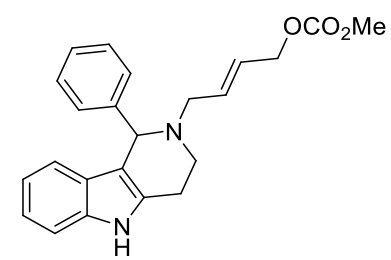


1a, viscous yellow oil. ${ }^{1} \mathrm{H}$ NMR (400 MHz, $\left.\mathrm{CDCl}_{3}\right) \delta 7.84(\mathrm{~s}, 1 \mathrm{H})$, 7.36-7.23 (m, $6 \mathrm{H}), 7.03(\mathrm{t}, J=7.6 \mathrm{~Hz}, 1 \mathrm{H}), 6.82(\mathrm{t}, J=7.2 \mathrm{~Hz}, 1 \mathrm{H}), 6.72(\mathrm{~d}, J=7.6 \mathrm{~Hz}, 1 \mathrm{H})$, 5.95-5.83 (m, 1H), 5.79-5.70 (m, 1H), $4.70(\mathrm{~s}, 1 \mathrm{H}), 4.62(\mathrm{~d}, J=6.0 \mathrm{~Hz}, 2 \mathrm{H}), 3.78(\mathrm{~s}$, 3H), 3.32 (dd, $J=14.4,4.4 \mathrm{~Hz}, 1 \mathrm{H}), 3.27-3.19$ (m, 1H), 3.07-2.95 (m, 2H), 2.87-2.71 $(\mathrm{m}, 2 \mathrm{H}) .{ }^{13} \mathrm{C}$ NMR $\left(101 \mathrm{MHz}, \mathrm{CDCl}_{3}\right) \delta 155.7,142.6,136.0,133.7,132.8,129.4$, 128.2, 127.4, 126.4, 126.2, 120.9, 119.1, 118.5, 111.2, 110.6, 68.1, 63.6, 55.1, 54.9, 47.0, 23.1. IR (thin film): $v_{\max }\left(\mathrm{cm}^{-1}\right)=3398,3056,3027,2953,2914,2807,1743$, 1618, 1595, 1491, 1447, 1379, 1326, 1259, 1099, 975, 940, 791, 741, 700. HRMS-ESI calcd for $\mathrm{C}_{23} \mathrm{H}_{25} \mathrm{~N}_{2} \mathrm{O}_{3}[\mathrm{M}+\mathrm{H}]^{+}:$377.1860, Found: 377.1863 .

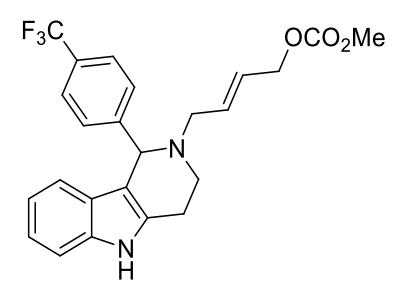

1a', viscous yellow oil. ${ }^{1} \mathrm{H} \mathrm{NMR}\left(400 \mathrm{MHz}, \mathrm{CDCl}_{3}\right) \delta 8.01(\mathrm{~s}, 1 \mathrm{H})$, 7.56-7.44 (m, 4H), $7.21(\mathrm{~d}, J=8.0 \mathrm{~Hz}, 1 \mathrm{H}), 7.04(\mathrm{t}, J=7.2 \mathrm{~Hz}, 1 \mathrm{H}), 6.85(\mathrm{t}, J=7.6 \mathrm{~Hz}, 1 \mathrm{H}), 6.74$ $(\mathrm{d}, J=8.0 \mathrm{~Hz}, 1 \mathrm{H}), 5.92-5.81(\mathrm{~m}, 1 \mathrm{H}), 5.79-5.68(\mathrm{~m}, 1 \mathrm{H}), 4.76(\mathrm{~s}, 1 \mathrm{H}), 4.62(\mathrm{~d}, J=$ $6.0 \mathrm{~Hz}, 2 \mathrm{H}), 3.77$ (s, 3H), 3.29-3.12 (m, 2H), 3.05-2.87 (m, 2H), 2.82-2.68 (m, 2H). ${ }^{19} \mathrm{~F} \mathrm{NMR}\left(376 \mathrm{MHz}, \mathrm{CDCl}_{3}\right) \delta-62.3$ (s). ${ }^{13} \mathrm{C} \mathrm{NMR}\left(101 \mathrm{MHz}, \mathrm{CDCl}_{3}\right) \delta 155.7,147.2$, $136.0,133.4,132.9,129.7,129.3,126.4,126.2,125.2(\mathrm{q}, J=3.8 \mathrm{~Hz}), 124.3(\mathrm{q}, J=$ $274.7 \mathrm{~Hz}), 121.3,119.5,118.3,110.7,110.5,68.0,62.8,55.1,54.9,46.8,22.9$. IR (thin film): $v_{\max }\left(\mathrm{cm}^{-1}\right)=3395,3058,2957,2814,1745,1615,1493,1447,1381.1322$, 1261, 1162, 1111, 1064, 1015, 974, 942, 841, 791, 742. HRMS-ESI calcd for $\mathrm{C}_{24} \mathrm{H}_{24} \mathrm{~F}_{3} \mathrm{~N}_{2} \mathrm{O}_{3}[\mathrm{M}+\mathrm{H}]^{+}$: 445.1734, Found: 445.1736.

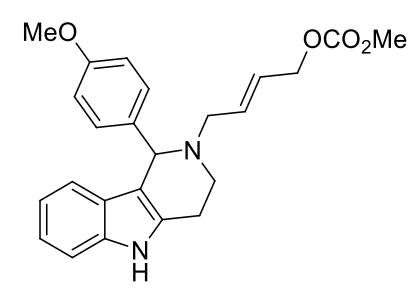

1a", viscous yellow oil. ${ }^{1} \mathrm{H}$ NMR (400 MHz, $\left.\mathrm{CDCl}_{3}\right) \delta 7.90$ (s, 1H), 7.31-7.15 (m, 3H), $7.02(\mathrm{t}, J=7.6 \mathrm{~Hz}, 1 \mathrm{H}), 6.90-6.78(\mathrm{~m}, 3 \mathrm{H}), 6.73(\mathrm{~d}, J=8.0 \mathrm{~Hz}, 1 \mathrm{H}), 5.93-5.83$ 
(m, 1H), 5.77-5.69 (m, 1H), 4.66-4.56 (m, 3H), 3.81-3.73 (m, 6H), $3.31(\mathrm{dd}, J=14.0$ $4.8 \mathrm{~Hz}, 1 \mathrm{H}), 3.25-3.19(\mathrm{~m}, 1 \mathrm{H}), 3.01-2.92(\mathrm{~m}, 2 \mathrm{H}), 2.83-2.61(\mathrm{~m}, 2 \mathrm{H}) .{ }^{13} \mathrm{C}$ NMR $(101$ $\left.\mathrm{MHz}, \mathrm{CDCl}_{3}\right) \delta 158.8,155.7,136.0,134.7,133.9,132.7,130.5,126.5,126.2,121.0$ $119.2,118.7,113.6,111.8,110.5,68.2,63.0,55.3,55.0,54.9,47.1,23.3$. IR (thin film): $v_{\max }\left(\mathrm{cm}^{-1}\right)=3398,3004,2954,2806,1744,1609,1587,1508,1446,1243$, $1174,1102,1031,976,940,833,792,741$. HRMS-ESI calcd for $\mathrm{C}_{24} \mathrm{H}_{27} \mathrm{~N}_{2} \mathrm{O}_{4}[\mathrm{M}+\mathrm{H}]^{+}$: 407.1965, Found: 407.1964.

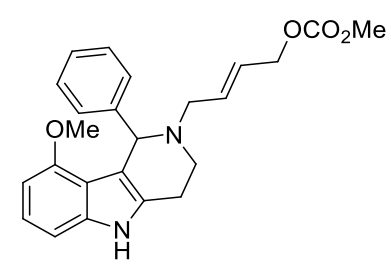

1b, viscous yellow oil. ${ }^{1} \mathrm{H}$ NMR (400 MHz, $\left.\mathrm{CDCl}_{3}\right) \delta 7.93(\mathrm{~s}, 1 \mathrm{H})$, 7.27-7.10 (m, $5 \mathrm{H}), 6.98(\mathrm{t}, J=8.0 \mathrm{~Hz}, 1 \mathrm{H}), 6.88(\mathrm{~d}, J=8.0 \mathrm{~Hz}, 1 \mathrm{H}), 6.34(\mathrm{~d}, J=7.6 \mathrm{~Hz}, 1 \mathrm{H})$, 6.04-5.92 (m, 1H), 5.78-5.69 (m, 1H), $5.23(\mathrm{~s}, 1 \mathrm{H}), 4.63(\mathrm{~d}, J=6.4 \mathrm{~Hz}, 2 \mathrm{H}), 3.77(\mathrm{~s}$, 3H), $3.46(\mathrm{~s}, 3 \mathrm{H}), 3.29(\mathrm{dd}, J=14.0,6.0 \mathrm{~Hz}, 1 \mathrm{H}), 3.18(\mathrm{dd}, J=14.0,6.0 \mathrm{~Hz}, 1 \mathrm{H})$, 3.06-2.95 (m, 1H), 2.92-2.79 (m, 2H), 2.62-2.50 (m, 1H). ${ }^{13} \mathrm{C}$ NMR (101 MHz, $\left.\mathrm{CDCl}_{3}\right) \delta 155.7,153.8,143.3,137.3,134.6,130.9,129.3,127.5,126.4,126.0,122.1$, $117.5,109.9,104.0,100.3,68.2,61.6,55.1,55.0,54.9,42.8,20.9$. IR (thin film): $v_{\max }$ $\left(\mathrm{cm}^{-1}\right)=3395,3024,2952,2930,2834,1744,1618,1596,1566,1507,1443,1378$, 1348, 1251, 1182, 1103, 976, 939, 840, 775, 733, 701. HRMS-ESI calcd for $\mathrm{C}_{24} \mathrm{H}_{27} \mathrm{~N}_{2} \mathrm{O}_{4}[\mathrm{M}+\mathrm{H}]^{+}:$407.1965, Found: 407.1968.

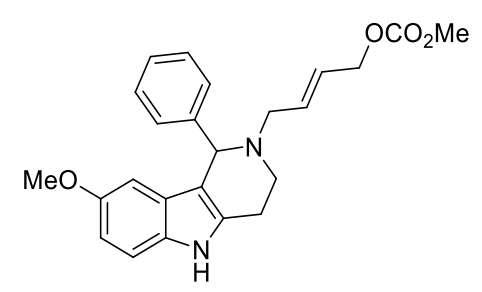

1c, viscous yellow oil. ${ }^{1} \mathrm{H}$ NMR $\left(400 \mathrm{MHz}, \mathrm{CDCl}_{3}\right) \delta 7.83(\mathrm{~s}, 1 \mathrm{H}), 7.43-7.19(\mathrm{~m}$, $5 \mathrm{H}), 7.09(\mathrm{~d}, J=8.8 \mathrm{~Hz}, 1 \mathrm{H}), 6.66(\mathrm{dd}, J=8.4,2.4 \mathrm{~Hz}, 1 \mathrm{H}), 6.14(\mathrm{~d}, J=2.0 \mathrm{~Hz}, 1 \mathrm{H})$, 5.93-5.82 (m, 1H), 5.78-5.67 (m, 1H), 4.69-4.55 (m, 3H), $3.77(\mathrm{~s}, 3 \mathrm{H}), 3.54(\mathrm{~s}, 3 \mathrm{H})$, $3.32(\mathrm{dd}, J=14.0,4.4 \mathrm{~Hz}, 1 \mathrm{H}), 3.26-3.16(\mathrm{~m}, 1 \mathrm{H}), 3.03-2.90(\mathrm{~m}, 2 \mathrm{H}), 2.81-2.66(\mathrm{~m}$, 
2H). ${ }^{13} \mathrm{C}$ NMR (101 MHz, $\left.\mathrm{CDCl}_{3}\right) \delta 155.7,153.6,142.7,133.8,133.5,131.1,129.5$, 128.3, 127.5, 126.9, 126.2, 111.5, 111.1, 110.5, 101.1, 68.2, 63.8, 55.6, 55.2, 54.9, 47.3, 23.4. IR (thin film): $v_{\max }\left(\mathrm{cm}^{-1}\right)=3398,2951,2830,1744,1627,1593,1481$, 1447, 1379, 1259, 1212, 1177, 1142, 1097, 1030, 975, 940, 842, 791, 743, 701. HRMS-ESI calcd for $\mathrm{C}_{24} \mathrm{H}_{27} \mathrm{~N}_{2} \mathrm{O}_{4}[\mathrm{M}+\mathrm{H}]^{+}: 407.1965$, Found: 407.1965.

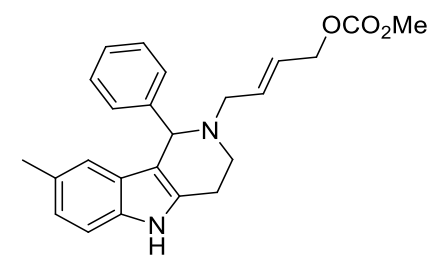

1d, viscous yellow oil. ${ }^{1} \mathrm{H}$ NMR (400 MHz, $\left.\mathrm{CDCl}_{3}\right) \delta 7.76(\mathrm{~s}, 1 \mathrm{H}), 7.36-7.21(\mathrm{~m}$, $5 \mathrm{H}), 7.09(\mathrm{~d}, J=8.0 \mathrm{~Hz}, 1 \mathrm{H}), 6.85(\mathrm{~d}, J=8.0 \mathrm{~Hz}, 1 \mathrm{H}), 6.55(\mathrm{~s}, 1 \mathrm{H}), 5.96-5.83(\mathrm{~m}$, 1H), 5.76-5.68 (m, 1H), $4.70(\mathrm{~s}, 1 \mathrm{H}), 4.61(\mathrm{~d}, J=6.0 \mathrm{~Hz}, 2 \mathrm{H}), 3.77$ (s, 3H), 3.29 (dd, $J=14.0,4.8 \mathrm{~Hz}, 1 \mathrm{H}), 3.22-3.13(\mathrm{~m}, 1 \mathrm{H}), 3.08-2.98(\mathrm{~m}, 1 \mathrm{H}), 2.95-2.83(\mathrm{~m}, 1 \mathrm{H})$, 2.80-2.67 (m, 2H), 2.21 (s, 3H). ${ }^{13} \mathrm{C}$ NMR (101 MHz, $\left.\mathrm{CDCl}_{3}\right) \delta$ 155.7, 142.7, 134.3, 134.0, 132.8, 129.4, 128.4, 128.2, 127.3, 126.8, 126.2, 122.5, 118.4, 110.7, 110.2, 68.2, 63.1, 55.1, 54.9, 46.6, 22.9, 21.5. IR (thin film): $v_{\max }\left(\mathrm{cm}^{-1}\right)=3378,3026,2915$, 2796, 1746, 1622, 1592, 1445, 1377, 1255, 1186, 1150, 1100, 974, 939, 869, 792, 736, 701. HRMS-ESI calcd for $\mathrm{C}_{24} \mathrm{H}_{27} \mathrm{~N}_{2} \mathrm{O}_{3}[\mathrm{M}+\mathrm{H}]^{+}$: 391.2016, Found: 391.2018 .

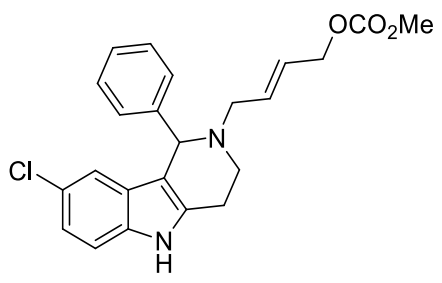

1e, viscous yellow oil. ${ }^{1} \mathrm{H}$ NMR (400 MHz, $\left.\mathrm{CDCl}_{3}\right) \delta 8.00(\mathrm{~s}, 1 \mathrm{H}), 7.38-7.21(\mathrm{~m}$, $5 \mathrm{H}), 7.11(\mathrm{~d}, J=8.8 \mathrm{~Hz}, 1 \mathrm{H}), 6.95(\mathrm{dd}, J=8.8,2.0 \mathrm{~Hz}, 1 \mathrm{H}), 6.67(\mathrm{~d}, J=1.6 \mathrm{~Hz}, 1 \mathrm{H})$, 5.94-5.80 (m, 1H), 5.78-5.67 (m, 1H), 4.69-4.55 (m, 3H), $3.78(\mathrm{~s}, 3 \mathrm{H}), 3.33-3.15(\mathrm{~m}$, 2H), 3.05-2.90 (m, 2H), 2.83-2.66 (m, 2H). ${ }^{13} \mathrm{C}$ NMR (101 MHz, $\left.\mathrm{CDCl}_{3}\right) \delta$ 155.7, 142.1, 134.4, 134.3, 133.7, 129.3, 128.4, 127.7, 127.6, 126.4, 124.9, 121.2, 118.1, 111.48, 111.46, 68.1, 63.3, 55.1, 54.9, 46.8, 23.3. IR (thin film): $v_{\max }\left(\mathrm{cm}^{-1}\right)=3409$, 3200, 3027, 2954, 2921, 2807, 1742, 1622, 1585, 1445, 1379, 1258, 1105, 1058, 1002, 
974, 940, 862, 791, 751, 701. HRMS-ESI calcd for $\mathrm{C}_{23} \mathrm{H}_{24} \mathrm{ClN}_{2} \mathrm{O}_{3}[\mathrm{M}+\mathrm{H}]^{+}$: 411.1470, Found: 411.1470.

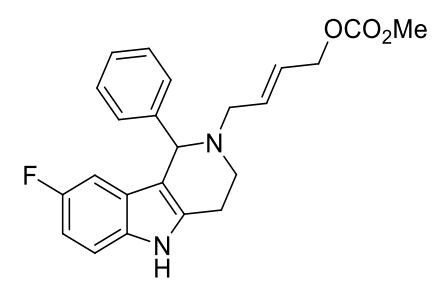

1f, viscous yellow oil. ${ }^{1} \mathrm{H}$ NMR $\left(400 \mathrm{MHz}, \mathrm{CDCl}_{3}\right) \delta 7.95(\mathrm{~s}, 1 \mathrm{H}), 7.39-7.22(\mathrm{~m}$, $5 \mathrm{H}), 7.10(\mathrm{dd}, J=8.8,4.4 \mathrm{~Hz}, 1 \mathrm{H}), 6.74(\mathrm{td}, J=8.8,2.4 \mathrm{~Hz}, 1 \mathrm{H}), 6.33(\mathrm{dd}, J=10.0$, $2.0 \mathrm{~Hz}, 1 \mathrm{H}), 5.92-5.81(\mathrm{~m}, 1 \mathrm{H}), 5.77-5.66(\mathrm{~m}, 1 \mathrm{H}), 4.64-4.57(\mathrm{~m}, 3 \mathrm{H}), 3.78(\mathrm{~s}, 3 \mathrm{H})$, 3.34-3.16 (m, 2H), 3.05-2.92 (m, 2H), 2.81-2.65 (m, 2H). ${ }^{19} \mathrm{~F}$ NMR (376 MHz, $\left.\mathrm{CDCl}_{3}\right)$ $\delta$-124.7 (m). ${ }^{13} \mathrm{C}$ NMR (101 MHz, $\left.\mathrm{CDCl}_{3}\right) \delta 157.5(\mathrm{~d}, J=233.3 \mathrm{~Hz}), 155.7,142.2$, 134.7, 133.6, 132.5, 129.4, 128.4, 127.7, 126.9 (d, $J=9.9 \mathrm{~Hz}), 126.3,112.0,111.0$ (d, $J=9.8 \mathrm{~Hz}), 109.0(\mathrm{~d}, J=26.1 \mathrm{~Hz}), 103.8(\mathrm{~d}, J=24.1 \mathrm{~Hz}), 68.1,63.7,55.1,54.9$, 47.2, 23.5. IR (thin film): $v_{\max }\left(\mathrm{cm}^{-1}\right)=3410,3062,3027,2954,2808,1742,1631$, 1588, 1483, 1448, 1379, 1260, 1177, 1139, 1094, 975, 937, 852, 789, 744, 701. HRMS-ESI calcd for $\mathrm{C}_{23} \mathrm{H}_{24} \mathrm{FN}_{2} \mathrm{O}_{3}[\mathrm{M}+\mathrm{H}]^{+}:$395.1765, Found: 395.1768 .

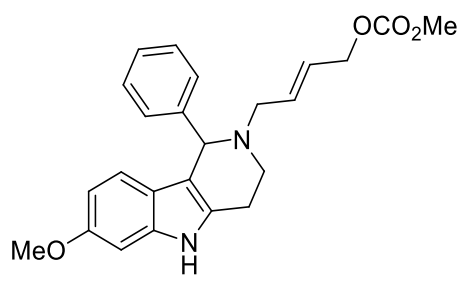

1g, viscous yellow oil. ${ }^{1} \mathrm{H}$ NMR $\left(400 \mathrm{MHz}, \mathrm{CDCl}_{3}\right) \delta 7.88(\mathrm{~s}, 1 \mathrm{H}), 7.33(\mathrm{dd}, J=$ 7.6, 1.6 Hz, 2H), 7.30-7.18 (m, 3H), $6.67(\mathrm{~d}, J=2.0 \mathrm{~Hz}, 1 \mathrm{H}), 6.56(\mathrm{~d}, J=8.8 \mathrm{~Hz}, 1 \mathrm{H})$, $6.48(\mathrm{dd}, J=8.8,2.4 \mathrm{~Hz}, 1 \mathrm{H}), 5.93-5.82(\mathrm{~m}, 1 \mathrm{H}), 5.77-5.66(\mathrm{~m}, 1 \mathrm{H}), 4.64(\mathrm{~s}, 1 \mathrm{H})$, $4.60(\mathrm{~d}, J=6.0 \mathrm{~Hz}, 2 \mathrm{H}), 3.76(\mathrm{~s}, 3 \mathrm{H}), 3.70(\mathrm{~s}, 3 \mathrm{H}), 3.29(\mathrm{dd}, J=14.0,4.4 \mathrm{~Hz}, 1 \mathrm{H})$, 3.22-3.14 (m, 1H), 3.02-2.85 (m, 2H), 2.75-2.63 (m, 2H). $\left.{ }^{13} \mathrm{C} \mathrm{NMR} \mathrm{(101} \mathrm{MHz,} \mathrm{CDCl}_{3}\right)$ $\delta 155.7,155.6,142.6,136.8,133.7,131.4,129.4,128.2,127.4,126.2,120.9,119.0$, 111.3, 108.4, 94.9, 68.1, 63.6, 55.6, 55.1, 54.8, 47.2, 23.2. IR (thin film): $v_{\max }\left(\mathrm{cm}^{-1}\right)=$ 3396, 2953, 2916, 2832, 1744, 1628, 1571, 1497, 1445, 1259, 1197, 1153, 1031, 940, 
819, 791, 755, 700. HRMS-ESI calcd for $\mathrm{C}_{24} \mathrm{H}_{27} \mathrm{~N}_{2} \mathrm{O}_{4}[\mathrm{M}+\mathrm{H}]^{+}:$407.1965, Found: 407.1963.

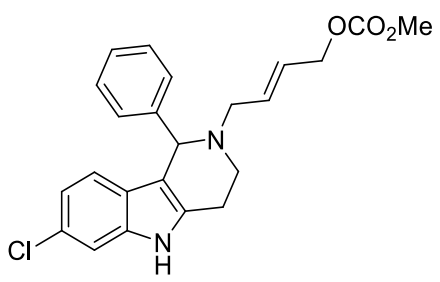

1h, viscous yellow oil. ${ }^{1} \mathrm{H}$ NMR (400 MHz, $\left.\mathrm{CDCl}_{3}\right) \delta 7.97$ (s, 1H), 7.35-7.23 (m, $5 \mathrm{H}), 7.12(\mathrm{~d}, J=1.6 \mathrm{~Hz}, 1 \mathrm{H}), 6.76(\mathrm{dd}, J=8.4,1.6 \mathrm{~Hz}, 1 \mathrm{H}), 6.56(\mathrm{~d}, J=8.8 \mathrm{~Hz}, 1 \mathrm{H})$, 5.92-5.79 (m, 1H), 5.76-5.67 (m, 1H), 4.67-4.53 (m, 3H), $3.77(\mathrm{~s}, 3 \mathrm{H}), 3.32-3.14(\mathrm{~m}$, 2H), 3.01-2.88 (m, 2H), 2.75-2.64 (m, 2H). ${ }^{13} \mathrm{C}$ NMR (101 MHz, $\left.\mathrm{CDCl}_{3}\right) \delta$ 155.7, $142.3,136.4,133.6,133.5,129.4,128.3,127.6,126.7,126.3,125.0,119.8,119.3$, 111.8, 110.5, 68.1, 63.5, 55.1, 54.9, 47.1, 23.3. IR (thin film): $v_{\max }\left(\mathrm{cm}^{-1}\right)=3405$, 2803, 1760, 1621, 1449, 1327, 1260, 1106, 1068, 976, 942, 912, 847, 818, 785, 754, 704, 620. HRMS-ESI calcd for $\mathrm{C}_{23} \mathrm{H}_{24} \mathrm{ClN}_{2} \mathrm{O}_{3}[\mathrm{M}+\mathrm{H}]^{+}:$411.1470, Found: 411.1468.

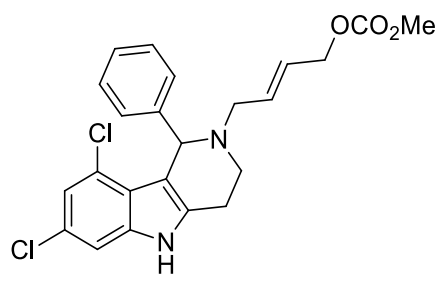

1i, viscous yellow oil. ${ }^{1} \mathrm{H}$ NMR $\left(400 \mathrm{MHz}, \mathrm{CDCl}_{3}\right) \delta 8.22(\mathrm{~s}, 1 \mathrm{H})$, 7.28-7.18 (m, 3H), 7.17-7.10 (m, 3H), $6.93(\mathrm{~s}, 1 \mathrm{H}), 6.06-5.97(\mathrm{~m}, 1 \mathrm{H}), 5.81-5.72(\mathrm{~m}, 1 \mathrm{H}), 5.46(\mathrm{~s}$, $1 \mathrm{H}), 4.66(\mathrm{~d}, J=6.4 \mathrm{~Hz}, 2 \mathrm{H}), 3.78(\mathrm{~s}, 3 \mathrm{H}), 3.37(\mathrm{dd}, J=13.6,5.6 \mathrm{~Hz}, 1 \mathrm{H}), 3.14(\mathrm{dd}$, $J=14.0,7.2 \mathrm{~Hz}, 1 \mathrm{H}), 3.01-2.81(\mathrm{~m}, 3 \mathrm{H}), 2.61-2.46(\mathrm{~m}, 1 \mathrm{H}) .{ }^{13} \mathrm{C}$ NMR $(101 \mathrm{MHz}$, $\left.\mathrm{CDCl}_{3}\right) \delta 155.7,142.0,137.0,134.5,134.2,129.4,127.8,127.05,127.01,126.6$, $125.9,123.6,120.5,109.7,109.4,68.1,60.1,55.0,54.9,41.4,20.4$. IR (thin film): $v_{\max }\left(\mathrm{cm}^{-1}\right)=3404,3026,2954,2929,2833,1743,1618,1552,1448,1414,1379$, 1323, 1257, 1181, 1082, 972, 941, 837, 789, 745, 701, 634. HRMS-ESI calcd for $\mathrm{C}_{23} \mathrm{H}_{23} \mathrm{Cl}_{2} \mathrm{~N}_{2} \mathrm{O}_{3}[\mathrm{M}+\mathrm{H}]^{+}:$445.1080, Found: 445.1086.

\section{3) Synthesis of Substrates $\mathbf{1 j}-1 \mathrm{~m}$}




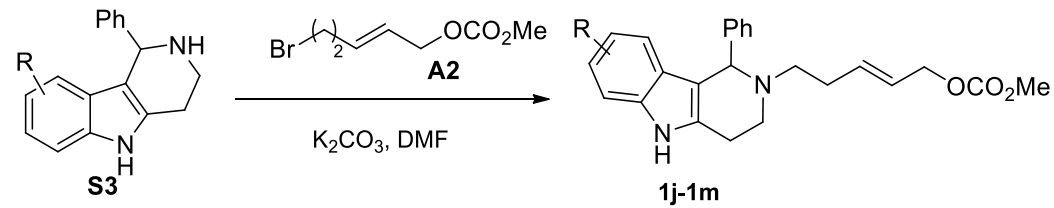

(E)-5-Bromopent-2-en-1-yl methyl carbonate (A2, $0.67 \mathrm{~g}, 3 \mathrm{mmol}, 1.5$ equiv) was added to a suspension of $\mathbf{S 3}$ (2 mmol, 1 equiv) and $\mathrm{K}_{2} \mathrm{CO}_{3}(0.55 \mathrm{~g}, 4 \mathrm{mmol}, 2$ equiv) in DMF (25 mL). The reaction mixture was stirred at $\mathrm{rt}$ for $12 \mathrm{~h}$, diluted with EtOAc $(30 \mathrm{~mL})$, and washed three times with brine $(50 \mathrm{~mL} \times 3)$. The organic phase was dried over $\mathrm{Na}_{2} \mathrm{SO}_{4}$, filtered and concentrated by rotary evaporation. Then the residue was purified by $\mathrm{Al}_{2} \mathrm{O}_{3}$ column chromatography $(\mathrm{PE} / \mathrm{EA}=4 / 1,2 / 1)$ to afford the desired products $\mathbf{1 j} \mathbf{j} \mathbf{1} \mathbf{m}$. The analytical data of the products are summarized below.

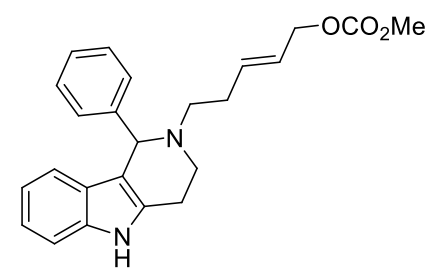

1j, viscous yellow oil. ${ }^{1} \mathrm{H}$ NMR $\left(400 \mathrm{MHz}, \mathrm{CDCl}_{3}\right) \delta 7.87(\mathrm{~s}, 1 \mathrm{H}), 7.33(\mathrm{~d}, J=$ $6.4 \mathrm{~Hz}, 2 \mathrm{H}), 7.30-7.16(\mathrm{~m}, 4 \mathrm{H}), 7.02(\mathrm{t}, J=7.2 \mathrm{~Hz}, 1 \mathrm{H}), 6.91-6.69(\mathrm{~m}, 2 \mathrm{H}), 5.76-5.66$ (m, 1H), 5.66-5.52 (m, 1H), $4.74(\mathrm{~s}, 1 \mathrm{H}), 4.52(\mathrm{~d}, J=6.4 \mathrm{~Hz}, 2 \mathrm{H}), 3.75(\mathrm{~s}, 3 \mathrm{H})$, 3.25-3.13 (m, 1H), 2.97-2.85 (m, 1H), 2.83-2.65 (m, 3H), 2.54-2.44 (m, 1H), 2.38-2.19 (m, 2H). ${ }^{13} \mathrm{C}$ NMR $\left(101 \mathrm{MHz}, \mathrm{CDCl}_{3}\right) \delta 155.7,142.9,136.0,135.3,132.7$, $129.4,128.2,127.3,126.6,124.2,121.0,119.3,118.6,111.4,110.5,68.7,63.0,54.8$, 52.5, 46.9, 30.3, 22.9. IR (thin film): $v_{\max }\left(\mathrm{cm}^{-1}\right)=3400,3056,3026,2952,2808$, 1743, 1673, 1621, 1593, 1490, 1448, 1378, 1326, 1258, 1121, 1096, 1016, 970, 938, 791, 741, 700. HRMS-ESI calcd for $\mathrm{C}_{24} \mathrm{H}_{27} \mathrm{~N}_{2} \mathrm{O}_{3}[\mathrm{M}+\mathrm{H}]^{+}$: 391.2016, Found: 391.2017.

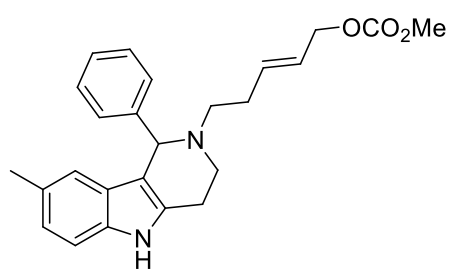


1k, viscous yellow oil. ${ }^{1} \mathrm{H}$ NMR (400 MHz, $\left.\mathrm{CDCl}_{3}\right) \delta 7.78(\mathrm{~s}, 1 \mathrm{H})$, 7.38-7.20 (m, $5 \mathrm{H}), 7.12(\mathrm{~d}, J=8.4 \mathrm{~Hz}, 1 \mathrm{H}), 6.86(\mathrm{~d}, J=8.4 \mathrm{~Hz}, 1 \mathrm{H}), 6.61(\mathrm{~s}, 1 \mathrm{H}), 5.81-5.71(\mathrm{~m}$, 1H), 5.62-5.51 (m, 1H), $4.76(\mathrm{~s}, 1 \mathrm{H}), 4.53(\mathrm{~d}, J=6.4 \mathrm{~Hz}, 2 \mathrm{H}), 3.76(\mathrm{~s}, 3 \mathrm{H}), 3.23-3.11$ (m, 1H), 2.90-2.76 (m, 3H), 2.74-2.65 (m, 1H), 2.61-2.49 (m, 1H), $2.32(\mathrm{~m}, 2 \mathrm{H}), 2.23$ (s, 3H). ${ }^{13} \mathrm{C}$ NMR $\left(101 \mathrm{MHz}, \mathrm{CDCl}_{3}\right) \delta 155.8,143.0,135.3,134.3,132.9,129.4$, $128.4,128.1,127.2,127.0,124.3,122.6,118.4,110.7,110.2$, 68.7, 62.5, 54.8, 52.5, 46.5, 30.5, 22.5, 21.5. IR (thin film): $v_{\max }\left(\mathrm{cm}^{-1}\right)=3397,3026,2958,2923,2854$, 1744, 1594, 1447, 1377, 1260, 1093, 1021, 971, 939, 795, 741, 700. HRMS-ESI calcd for $\mathrm{C}_{25} \mathrm{H}_{29} \mathrm{~N}_{2} \mathrm{O}_{3}[\mathrm{M}+\mathrm{H}]^{+}$: 405.2173, Found: 405.2168.

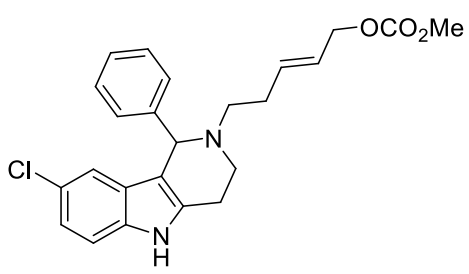

11, viscous yellow oil. ${ }^{1} \mathrm{H}$ NMR (400 MHz, $\left.\mathrm{CDCl}_{3}\right) \delta 8.03(\mathrm{~s}, 1 \mathrm{H}), 7.34-7.17(\mathrm{~m}$, 5H), $7.07(\mathrm{~d}, J=8.4 \mathrm{~Hz}, 1 \mathrm{H}), 6.94(\mathrm{~d}, J=8.4 \mathrm{~Hz}, 1 \mathrm{H}), 6.72(\mathrm{~s}, 1 \mathrm{H}), 5.75-5.65(\mathrm{~m}$, 1H), 5.62-5.46 (m, 1H), $4.68(\mathrm{~s}, 1 \mathrm{H}), 4.52$ (d, $J=6.4 \mathrm{~Hz}, 2 \mathrm{H}), 3.75(\mathrm{~s}, 3 \mathrm{H}), 3.24-3.10$ (m, 1H), 2.96-2.83 (m, 1H), 2.82-2.58 (m, 3H), 2.54-2.41 (m, 1H), 2.37-2.15 (m, 2H). ${ }^{13} \mathrm{C}$ NMR (101 MHz, $\left.\mathrm{CDCl}_{3}\right) \delta 155.7,142.3,135.2,134.4,134.3,129.2,128.3,127.7$, $127.5,124.8,124.3,121.1,118.0,111.5,111.3,68.6,62.8,54.8,52.5,46.6,30.2,22.9$. IR (thin film): $v_{\max }\left(\mathrm{cm}^{-1}\right)=3409,3027,2952,2808,1739,1672,1585,1446,1378$, 1259, 1104, 1059, 1002, 970, 937, 863, 792, 750, 701. HRMS-ESI calcd for $\mathrm{C}_{24} \mathrm{H}_{26} \mathrm{ClN}_{2} \mathrm{O}_{3}[\mathrm{M}+\mathrm{H}]^{+}:$425.1626, Found: 425.1629 .

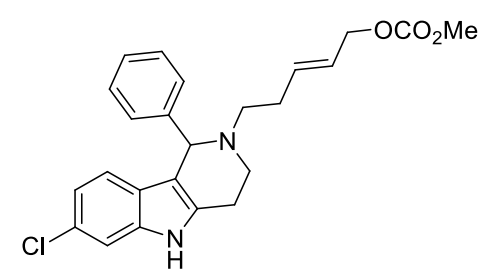

1m, viscous yellow oil. ${ }^{1} \mathrm{H} \mathrm{NMR}\left(400 \mathrm{MHz}, \mathrm{CDCl}_{3}\right) \delta 7.92(\mathrm{~s}, 1 \mathrm{H})$, 7.38-7.23 (m, $6 \mathrm{H}), 6.78(\mathrm{~d}, J=8.4 \mathrm{~Hz}, 1 \mathrm{H}), 6.62(\mathrm{~d}, J=8.4 \mathrm{~Hz}, 1 \mathrm{H}), 5.77-5.66(\mathrm{~m}, 1 \mathrm{H}), 5.61-5.50$ $(\mathrm{m}, 1 \mathrm{H}), 4.68(\mathrm{~s}, 1 \mathrm{H}), 4.52(\mathrm{~d}, J=6.4 \mathrm{~Hz}, 2 \mathrm{H}), 3.76(\mathrm{~s}, 3 \mathrm{H}), 3.24-3.16(\mathrm{~m}, 1 \mathrm{H})$, 
3.00-2.90 (m, 1H), 2.85-2.75 (m, 2H), 2.73-2.62 (m, 1H), 2.53-2.43 (m, 1H),

2.38-2.19 (m, 2H). ${ }^{13} \mathrm{C}$ NMR (101 MHz, $\left.\mathrm{CDCl}_{3}\right) \delta 155.8,142.6,136.4,135.2,133.5$, $129.4,128.3,127.5,126.9,125.2,124.3,119.9,119.4,111.9,110.6,68.7,63.2,54.9$, 52.6, 47.0, 30.3, 23.2. IR (thin film): $v_{\max }\left(\mathrm{cm}^{-1}\right)=3409,3062,3027,2953,2917$, 2812, 1742, 1673, 1622, 1561, 1446, 1378, 1324, 1260, 1129, 1097, 1064, 971, 938, 909, 843, 792, 748, 700. HRMS-ESI calcd for $\mathrm{C}_{24} \mathrm{H}_{26} \mathrm{ClN}_{2} \mathrm{O}_{3}[\mathrm{M}+\mathrm{H}]^{+}$: 425.1626, Found: 425.1624.

\section{4) Synthesis of Substrates 1n-1q}

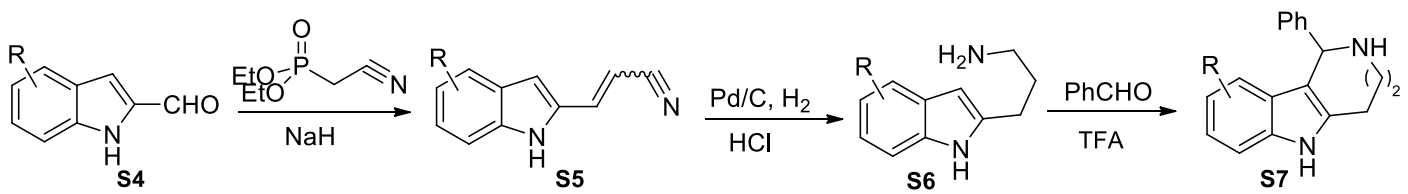<smiles>[R]c1ccc2[nH]c3c(c2c1)C(c1ccccc1)N(C/C=C/COC(C)=O)CC3</smiles>

Indole-2-carboxaldehyde derivatives $\mathbf{S} \mathbf{4}$ were obtained according to Lit. ${ }^{2}$

Diethylcyanomethylphosphonate $(2.15 \mathrm{~mL}, 13.3 \mathrm{mmol}, 1$ equiv) was slowly added to a suspension of $\mathrm{NaH}(60 \%, 1.27 \mathrm{~g}, 31.8 \mathrm{mmol}, 2.1$ equiv) in THF (40 mL) at $0{ }^{\circ} \mathrm{C}$ and the resulting mixture was stirred at $\mathrm{rt}$ for $30 \mathrm{~min}$. A solution of $\mathbf{S 4}$ (13.3 mmol, 1 equiv) in THF (15 mL) was then slowly added. The reaction mixture was stirred for another $10 \mathrm{~h}$. Then the reaction mixture was quenched with water and extracted with diethyl ether. The organic layer was washed with brine, dried over $\mathrm{Na}_{2} \mathrm{SO}_{4}$, filtered and concentrated by rotary evaporation. The residue was purified by silica gel column chromatography $(\mathrm{PE} / \mathrm{EA}=5 / 1)$ to obtain the crude product $\mathbf{S 5}$.

The above obtained crude product $\mathbf{S 5}$ ( $8.32 \mathrm{mmol}, 1$ equiv) was added into a flask containing methanol $(40 \mathrm{~mL})$, hydrogen chloride $(12 \mathrm{M}, 3.6 \mathrm{~mL})$ and $\mathrm{Pd} / \mathrm{C}(1.1 \mathrm{~g}$, palladium on activated carbon, $10 \% \mathrm{Pd}$ basis, 0.1 equiv). The reaction mixture was stirred under $\mathrm{H}_{2}$ atmosphere (1 atm) at $\mathrm{rt}$ over $48 \mathrm{~h}$. Then the catalyst was filtered off 
and the solvent was evaporated. The residue was basified with sodium hydroxide solution, and extracted with $\mathrm{CH}_{2} \mathrm{Cl}_{2}$. The residue was dried over $\mathrm{Na}_{2} \mathrm{SO}_{4}$, filtered, and evaporated to yield a crude oil S6.

Trifluoroacetic acid (0.93 mL, $12.5 \mathrm{mmol}, 1.5$ equiv) was added to a solution of above obtained S6 and benzaldehyde (1.7 mL, $16.6 \mathrm{mmol}, 2$ equiv) in THF (20 mL). The reaction mixture was stirred at $\mathrm{rt}$ for $5 \mathrm{~h}$, then quenched with a $10 \%$ $\mathrm{K}_{2} \mathrm{CO}_{3}$ aqueous solution $(30 \mathrm{~mL})$ and extracted with $\mathrm{CH}_{2} \mathrm{Cl}_{2}$. The organic layer was concentrated by rotary evaporation and purified by $\mathrm{Al}_{2} \mathrm{O}_{3}$ column chromatography $(\mathrm{DCM} / \mathrm{MeOH}=50 / 1,20 / 1)$ to afford the crude product $\mathbf{S 7}$.

(E)-4-Bromobut-2-en-1-yl methyl carbonate (A1, $2.1 \mathrm{~g}, 1.2$ equiv, $10 \mathrm{mmol}$ ) was added to a solution of $\mathbf{S 7}$ (1 equiv, $8.32 \mathrm{mmol}$ ) and $\mathrm{Et}_{3} \mathrm{~N}$ (3.5 ml, $25 \mathrm{mmol}, 3$ equiv) in THF $(40 \mathrm{ml})$ at $0{ }^{\circ} \mathrm{C}$. Then the ice bath was removed and the reaction mixture was stirred at rt until the starting material was consumed completely (monitored by TLC). The crude reaction mixture was quenched with water and extracted with EtOAc. The organic layer was washed with brine, dried over $\mathrm{Na}_{2} \mathrm{SO}_{4}$ and concentrated by rotary evaporation. Then the residue was purified by $\mathrm{Al}_{2} \mathrm{O}_{3}$ column chromatography (PE/EA $=4 / 1,2 / 1)$ to afford the desired products 1n-1q. The analytical data of the products are summarized below.

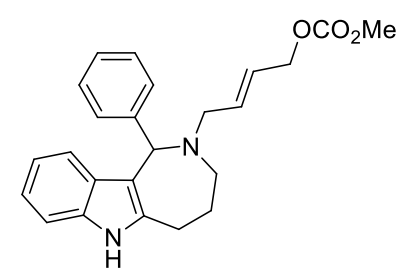

1n, viscous yellow oil. ${ }^{1} \mathrm{H} \mathrm{NMR}\left(400 \mathrm{MHz}, \mathrm{CDCl}_{3}\right) \delta 7.97(\mathrm{~s}, 1 \mathrm{H}), 7.32-7.13(\mathrm{~m}$, 7H), 7.09 (t, $J=7.2 \mathrm{~Hz}, 1 \mathrm{H}), 6.99(\mathrm{t}, J=7.2 \mathrm{~Hz}, 1 \mathrm{H}), 6.06-5.93(\mathrm{~m}, 1 \mathrm{H}), 5.70-5.57$ (m, 1H), $5.39(\mathrm{~s}, 1 \mathrm{H}), 4.63(\mathrm{~d}, J=6.2 \mathrm{~Hz}, 2 \mathrm{H}), 3.78(\mathrm{~s}, 3 \mathrm{H}), 3.34-3.23(\mathrm{~m}, 2 \mathrm{H})$, $3.10-2.85(\mathrm{~m}, 3 \mathrm{H}), 2.77-2.67(\mathrm{~m}, 1 \mathrm{H}), 2.03-1.88(\mathrm{~m}, 1 \mathrm{H}), 1.45-1.33(\mathrm{~m}, 1 \mathrm{H}) .{ }^{13} \mathrm{C}$ NMR $\left(101 \mathrm{MHz}, \mathrm{CDCl}_{3}\right) \delta 155.7,142.3,137.0,134.9,134.7,130.0,128.7,128.1$, 126.6, 126.0, 121.0, 119.4, 117.7, 112.0, 110.4, 68.2, 59.8, 54.9, 54.3, 50.0, 28.5, 21.7. IR (thin film): $v_{\max }\left(\mathrm{cm}^{-1}\right)=3398,3025,2923,2849,1744,1489,1444,1370,1333$, 
1257, 1189, 1142, 1109, 1018, 975, 939, 851, 789, 737, 699, 618. HRMS-ESI calcd for $\mathrm{C}_{24} \mathrm{H}_{27} \mathrm{~N}_{2} \mathrm{O}_{3}[\mathrm{M}+\mathrm{H}]^{+}$: 391.2016, Found: 391.2020.

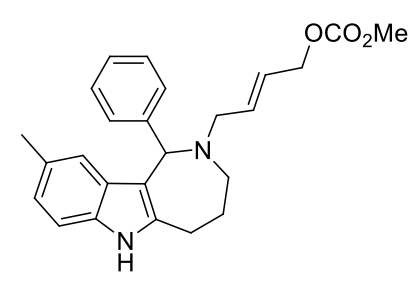

1o, viscous yellow oil. ${ }^{1} \mathrm{H}$ NMR (400 $\left.\mathrm{MHz}, \mathrm{CDCl}_{3}\right) \delta 7.88(\mathrm{~s}, 1 \mathrm{H})$, 7.29-7.09 (m, 6H), $7.06(\mathrm{~s}, 1 \mathrm{H}), 6.90(\mathrm{~d}, J=8.4 \mathrm{~Hz}, 1 \mathrm{H}), 6.04-5.93(\mathrm{~m}, 1 \mathrm{H}), 5.67-5.56(\mathrm{~m}, 1 \mathrm{H})$, $5.36(\mathrm{~s}, 1 \mathrm{H}), 4.63(\mathrm{~d}, J=6.4 \mathrm{~Hz}, 2 \mathrm{H}), 3.75(\mathrm{~s}, 3 \mathrm{H}), 3.31-3.18(\mathrm{~m}, 2 \mathrm{H}), 3.07-2.76(\mathrm{~m}$, $3 \mathrm{H}), 2.68-2.59(\mathrm{~m}, 1 \mathrm{H}), 2.35(\mathrm{~s}, 3 \mathrm{H}), 1.99-1.82(\mathrm{~m}, 1 \mathrm{H}), 1.38-1.29(\mathrm{~m}, 1 \mathrm{H}) .{ }^{13} \mathrm{C} \mathrm{NMR}$ $\left(101 \mathrm{MHz}, \mathrm{CDCl}_{3}\right) \delta 155.7,142.3,137.1,134.9,132.9,130.2,128.7,128.4,128.0$, $126.5,125.9,122.4,117.3,111.3,110.1,68.2,59.7,54.8,54.2,49.9,28.4,21.50$, 21.47. IR (thin film): $v_{\max }\left(\mathrm{cm}^{-1}\right)=3394,3024,2919,2851,1745,1587,1485,1444$, 1369, 1257, 1109, 1023, 941, 789, 730, 699, 628. HRMS-ESI calcd for $\mathrm{C}_{25} \mathrm{H}_{29} \mathrm{~N}_{2} \mathrm{O}_{3}$ $[\mathrm{M}+\mathrm{H}]^{+}:$405.2173, Found: 405.2178 .

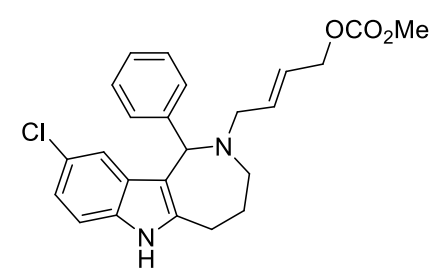

1p, viscous yellow oil. ${ }^{1} \mathrm{H}$ NMR (400 MHz, $\left.\mathrm{CDCl}_{3}\right) \delta 8.05(\mathrm{~s}, 1 \mathrm{H}), 7.32-7.13(\mathrm{~m}$, 7H), $7.03(\mathrm{dd}, J=8.8,2.0 \mathrm{~Hz}, 1 \mathrm{H}), 6.07-5.93(\mathrm{~m}, 1 \mathrm{H}), 5.75-5.57(\mathrm{~m}, 1 \mathrm{H}), 5.29$ (s, $1 \mathrm{H}), 4.65(\mathrm{~d}, J=6.0 \mathrm{~Hz}, 2 \mathrm{H}), 3.79(\mathrm{~s}, 3 \mathrm{H}), 3.28(\mathrm{~d}, J=6.4 \mathrm{~Hz}, 2 \mathrm{H}), 3.07-2.86(\mathrm{~m}$, $3 \mathrm{H}), 2.79-2.66(\mathrm{~m}, 1 \mathrm{H}), 2.02-1.87(\mathrm{~m}, 1 \mathrm{H}), 1.48-1.34(\mathrm{~m}, 1 \mathrm{H}) .{ }^{13} \mathrm{C}$ NMR (101 MHz, $\left.\mathrm{CDCl}_{3}\right) \delta 155.8,141.8,138.6,134.7,133.0,131.0,128.7,128.2,126.8,126.1,125.2$, $121.2,117.2,112.1,111.5,68.1,60.0,54.9,54.4,49.7,28.5,21.6$. IR (thin film): $v_{\max }$ $\left(\mathrm{cm}^{-1}\right)=3408,3025,2925,2849,1742,1575,1444,1372,1257,1146,1110,1060$, 1022, 966, 941, 858, 789, 731, 701, 627. HRMS-ESI calcd for $\mathrm{C}_{24} \mathrm{H}_{26} \mathrm{ClN}_{2} \mathrm{O}_{3}[\mathrm{M}+\mathrm{H}]^{+}$: 425.1626, Found: 425.1628. 


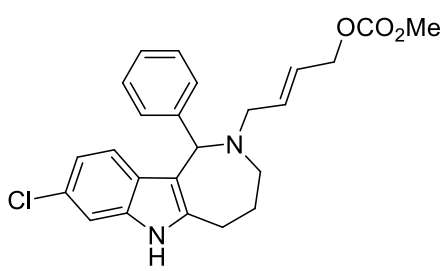

1q, viscous yellow oil. ${ }^{1} \mathrm{H} \mathrm{NMR}\left(400 \mathrm{MHz}, \mathrm{CDCl}_{3}\right) \delta 8.17(\mathrm{~s}, 1 \mathrm{H})$, 7.34-7.08 (m, $7 \mathrm{H}), 6.94(\mathrm{dd}, J=8.4,1.6 \mathrm{~Hz}, 1 \mathrm{H}), 6.03-5.93(\mathrm{~m}, 1 \mathrm{H}), 5.65-5.53(\mathrm{~m}, 1 \mathrm{H}), 5.33(\mathrm{~s}$, $1 \mathrm{H}), 4.63(\mathrm{~d}, J=6.0 \mathrm{~Hz}, 2 \mathrm{H}), 3.78(\mathrm{~s}, 3 \mathrm{H}), 3.26(\mathrm{~d}, J=6.4 \mathrm{~Hz}, 2 \mathrm{H}), 3.06-2.80(\mathrm{~m}$, $3 \mathrm{H}), 2.75-2.65(\mathrm{~m}, 1 \mathrm{H}), 2.01-1.85(\mathrm{~m}, 1 \mathrm{H}), 1.48-1.32(\mathrm{~m}, 1 \mathrm{H}) .{ }^{13} \mathrm{C}$ NMR $(101 \mathrm{MHz}$, $\left.\mathrm{CDCl}_{3}\right) \delta 155.7,141.9,137.8,135.0,134.8,128.6,128.4,128.1,126.7,126.6,126.1$, $119.9,118.5,112.1,110.5,68.1,59.6,54.9,54.3,49.9,28.3,21.5$. IR (thin film): $v_{\max }$ $\left(\mathrm{cm}^{-1}\right)=3407,3057,3025,2923,2850,1744,1619,1600,1556,1489,1444,1369$, 1257, 1110, 1061, 1023, 939, 851, 791, 733, 700. HRMS-ESI calcd for $\mathrm{C}_{24} \mathrm{H}_{26} \mathrm{ClN}_{2} \mathrm{O}_{3}$ $[\mathrm{M}+\mathrm{H}]^{+}:$425.1626, Found: 425.1633 .

\section{5) Synthesis of Substrates 1r-1t}
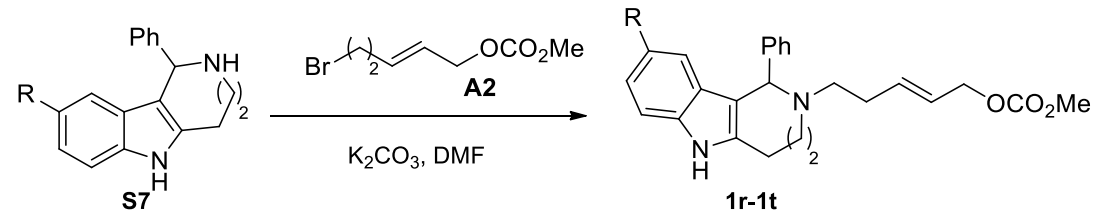

(E)-5-bromopent-2-en-1-yl methyl carbonate (A2, $0.67 \mathrm{~g}, 3$ mmol, 1.5 equiv) was added to a solution of $\mathbf{S} 7\left(0.52 \mathrm{~g}, 2 \mathrm{mmol}, 1\right.$ equiv) and $\mathrm{K}_{2} \mathrm{CO}_{3}(0.55 \mathrm{~g}, 4 \mathrm{mmol}, 2$ equiv) in DMF (25 mL). The reaction mixture was stirred at $\mathrm{rt}$ for $12 \mathrm{~h}$, diluted with EtOAc $(30 \mathrm{~mL})$, and washed three times with brine $(3 \times 50 \mathrm{~mL})$. The organic phase was dried over $\mathrm{Na}_{2} \mathrm{SO}_{4}$, filtered and concentrated by rotary evaporation. Then the residue was purified by $\mathrm{Al}_{2} \mathrm{O}_{3}$ column chromatography $(\mathrm{PE} / \mathrm{EA}=4 / 1,2 / 1)$ to afford the desired product 1r-1t.<smiles>CC(=O)OC/C=C/CCN1C=[C]c2[nH]c3ccccc3c2C1c1ccccc1</smiles> 
1r, viscous yellow oil. ${ }^{1} \mathrm{H}$ NMR (400 MHz, $\left.\mathrm{CDCl}_{3}\right) \delta 7.93(\mathrm{~s}, 1 \mathrm{H}), 7.40-7.13(\mathrm{~m}$, $7 \mathrm{H}), 7.10(\mathrm{t}, J=7.6 \mathrm{~Hz}, 1 \mathrm{H}), 7.01(\mathrm{t}, J=7.6 \mathrm{~Hz}, 1 \mathrm{H}), 5.96-5.85(\mathrm{~m}, 1 \mathrm{H}), 5.74-5.61$ $(\mathrm{m}, 1 \mathrm{H}), 5.38(\mathrm{~s}, 1 \mathrm{H}), 4.59(\mathrm{~d}, J=6.8 \mathrm{~Hz}, 2 \mathrm{H}), 3.76(\mathrm{~s}, 3 \mathrm{H}), 3.02-2.85(\mathrm{~m}, 3 \mathrm{H})$, 2.84-2.63 (m, 3H), 2.45-2.29 (m, 2H), 2.06-1.91 (m, 1H), 1.44-1.33 (m, 1H). ${ }^{13} \mathrm{C}$ NMR $\left(101 \mathrm{MHz}, \mathrm{CDCl}_{3}\right) \delta 155.8,142.5,136.8,135.9,134.7,130.2,128.7,128.1$, 126.5, 124.2, 121.0, 119.5, 117.5, 112.4, 110.5, 68.8, 60.2, 54.8, 51.5, 49.6, 31.5, 28.5, 21.4. $v_{\max }\left(\mathrm{cm}^{-1}\right)=3405,3056,3026,2931,2849,1746,1585,1489,1445,1379$, 1333, 1264, 1187, 1112, 1027, 856, 793, 740, 700, 620. HRMS-ESI calcd for $\mathrm{C}_{25} \mathrm{H}_{29} \mathrm{~N}_{2} \mathrm{O}_{3}[\mathrm{M}+\mathrm{H}]^{+}:$405.2173, Found: 405.2174.

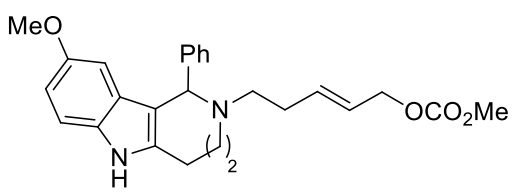

1s, viscous yellow oil. ${ }^{1} \mathrm{H}$ NMR (400 MHz, $\left.\mathrm{CDCl}_{3}\right) \delta 8.02(\mathrm{~s}, 1 \mathrm{H}), 7.33-7.05(\mathrm{~m}$, $6 \mathrm{H}), 6.82-6.68(\mathrm{~m}, 2 \mathrm{H}), 5.97-5.85(\mathrm{~m}, 1 \mathrm{H}), 5.75-5.57(\mathrm{~m}, 1 \mathrm{H}), 5.32(\mathrm{~s}, 1 \mathrm{H}), 4.59$ (d, $J$ $=6.4 \mathrm{~Hz}, 2 \mathrm{H}), 3.73(\mathrm{~s}, 6 \mathrm{H}), 3.05-2.56(\mathrm{~m}, 6 \mathrm{H}), 2.45-2.21(\mathrm{~m}, 2 \mathrm{H}), 2.00-1.84(\mathrm{~m}, 1 \mathrm{H})$, 1.39-1.23 (m, 1H). ${ }^{13} \mathrm{C}$ NMR (101 MHz, $\left.\mathrm{CDCl}_{3}\right) \delta 155.7,153.9,142.4,137.8,135.8$, 130.6, 129.7, 128.6, 128.0, 126.4, 124.0, 111.9, 111.1, 110.4, 99.6, 68.7, 60.3, 55.8, 54.7, 51.3, 49.4, 31.4, 28.4, 21.2. $v_{\max }\left(\mathrm{cm}^{-1}\right)=3402,2930,2833,1744,1624,1586$, 1483, 1448, 1379, 1259, 1211, 1172, 1111, 1028, 939, 859, 789, 730, 699, 626. HRMS-ESI calcd for $\mathrm{C}_{26} \mathrm{H}_{31} \mathrm{~N}_{2} \mathrm{O}_{4}[\mathrm{M}+\mathrm{H}]^{+}$: 435.2278, Found: 435.2278.

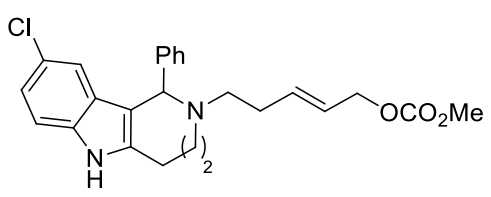

1t, viscous yellow oil. ${ }^{1} \mathrm{H}$ NMR (400 MHz, $\left.\mathrm{CDCl}_{3}\right) \delta 8.11(\mathrm{~s}, 1 \mathrm{H}), 7.30-7.12(\mathrm{~m}$, 7H), $7.02(\mathrm{dd}, J=8.8,2.0 \mathrm{~Hz}, 1 \mathrm{H}), 5.96-5.83(\mathrm{~m}, 1 \mathrm{H}), 5.74-5.61(\mathrm{~m}, 1 \mathrm{H}), 5.29(\mathrm{~s}$, $1 \mathrm{H}), 4.60(\mathrm{~d}, J=6.4 \mathrm{~Hz}, 2 \mathrm{H}), 3.76(\mathrm{~s}, 3 \mathrm{H}), 2.99-2.82(\mathrm{~m}, 3 \mathrm{H}), 2.79-2.61(\mathrm{~m}, 3 \mathrm{H})$, 2.43-2.24 (m, 2H), 2.02-1.84 (m, 1H), 1.42-1.30 (m, 1H). ${ }^{13} \mathrm{C}$ NMR (101 MHz, $\left.\mathrm{CDCl}_{3}\right) \delta 155.7,142.0,138.5,135.7,133.0,131.2,128.6,128.1,126.7,125.1,124.1$, $121.0,116.9,112.1,111.5,68.8,60.3,54.8,51.4,49.3,31.4,28.4,21.1 . v_{\max }\left(\mathrm{cm}^{-1}\right)=$ 
$3408,2930,2848,1742,1575,1443,1379,1258,1114,1026,939,861,789,731,701$, 627. HRMS-ESI calcd for $\mathrm{C}_{25} \mathrm{H}_{28} \mathrm{ClN}_{2} \mathrm{O}_{3}[\mathrm{M}+\mathrm{H}]^{+}$: 439.1783, Found: 439.1783 .

\section{6) Synthesis of Substrates 1u-1v}

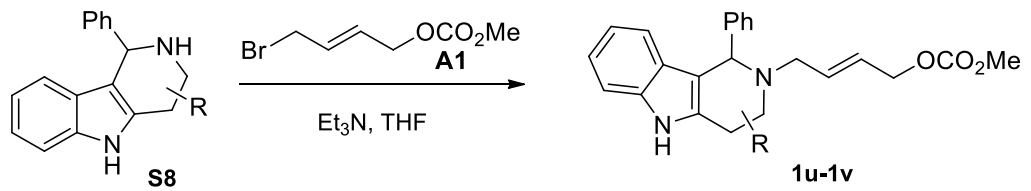

(E)-4-Bromobut-2-en-1-yl methyl carbonate (A1, $2.1 \mathrm{~g}, 1.2$ equiv, $10 \mathrm{mmol}$ ) was added to a solution of $\mathbf{S 8}$ ( 1 equiv, $8.32 \mathrm{mmol}$ ) and $\mathrm{Et}_{3} \mathrm{~N}$ (3.5 ml, $25 \mathrm{mmol}, 3$ equiv) in THF (40 ml) at $0{ }^{\circ} \mathrm{C}$. Then the ice bath was removed and the reaction mixture was stirred at rt until the starting material was consumed completely (monitored by TLC). The crude reaction mixture was quenched with water and extracted with EtOAc. The organic layer was washed with brine, dried over $\mathrm{Na}_{2} \mathrm{SO}_{4}$ and concentrated by rotary evaporation. Then the residue was purified by $\mathrm{Al}_{2} \mathrm{O}_{3}$ column chromatography (PE/EA $=4 / 1,2 / 1)$ to afford the desired products $\mathbf{1 u}-\mathbf{1 v}$.<smiles>CC(=O)OC/C=C/CN1C[C@H](c2ccccc2)c2[nH]c3ccccc3c2C1c1ccccc1</smiles>

1u, viscous yellow oil. ${ }^{1} \mathrm{H}$ NMR $\left(400 \mathrm{MHz}, \mathrm{CDCl}_{3}\right) \delta 7.55(\mathrm{~s}, 1 \mathrm{H}), 7.42(\mathrm{~d}, J=$ $7.2 \mathrm{~Hz}, 2 \mathrm{H}), 7.37-7.21(\mathrm{~m}, 8 \mathrm{H}), 7.06(\mathrm{~d}, J=8.0 \mathrm{~Hz}, 1 \mathrm{H}), 6.95(\mathrm{t}, J=7.6 \mathrm{~Hz}, 1 \mathrm{H})$, $6.75(\mathrm{t}, J=7.6 \mathrm{~Hz}, 1 \mathrm{H}), 6.61(\mathrm{~d}, J=8.0 \mathrm{~Hz}, 1 \mathrm{H}), 5.87-5.75(\mathrm{~m}, 1 \mathrm{H}), 5.70-5.59(\mathrm{~m}$, $1 \mathrm{H}), 4.72(\mathrm{~s}, 1 \mathrm{H}), 4.56-4.48(\mathrm{~m}, 3 \mathrm{H}), 3.71(\mathrm{~s}, 3 \mathrm{H}), 3.45-3.29(\mathrm{~m}, 2 \mathrm{H}), 2.88(\mathrm{dd}, J=$ 14.4, 8.0 Hz, 1H), $2.69(\mathrm{t}, J=10.8 \mathrm{~Hz}, 1 \mathrm{H}) .{ }^{13} \mathrm{C} \mathrm{NMR}\left(101 \mathrm{MHz}, \mathrm{CDCl}_{3}\right) \delta$ 155.6, $142.7,140.8,136.2,135.0,133.1,129.6,128.9,128.4,127.7,127.5,126.5,126.0$, $121.2,119.3,119.0,113.8,110.7,68.0,65.2,58.3,55.0,54.8,42.7$. IR (thin film): $v_{\max }\left(\mathrm{cm}^{-1}\right)=3398,3058,3027,2953,2801,1742,1491,1448,1260,1097,1017,940$, 907, 843, 791, 741, 699. HRMS-ESI calcd for $\mathrm{C}_{29} \mathrm{H}_{29} \mathrm{~N}_{2} \mathrm{O}_{3}[\mathrm{M}+\mathrm{H}]^{+}$: 453.2173, Found: 453.2175 . 


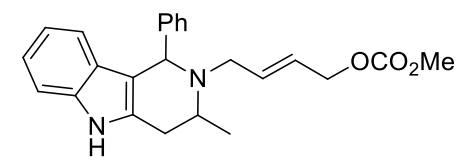

1v, viscous yellow oil, $\mathrm{dr}=3.2 / 1 .{ }^{1} \mathrm{H} \mathrm{NMR}\left(400 \mathrm{MHz}, \mathrm{CDCl}_{3}\right) \delta 7.84(\mathrm{~s}, 1 \mathrm{H})$, 7.42-7.35 (m, 2H), 7.27-7.21 (m, 3H), $7.15(\mathrm{~d}, J=8.0 \mathrm{~Hz}, 1 \mathrm{H}), 7.01-6.94(\mathrm{~m}, 1 \mathrm{H})$, $6.78(\mathrm{t}, J=7.6 \mathrm{~Hz}, 1 \mathrm{H}), 6.64(\mathrm{~d}, J=7.6 \mathrm{~Hz}, 1 \mathrm{H}), 6.13-5.97(\mathrm{~m}, 1 \mathrm{H}), 5.78-5.64(\mathrm{~m}$, 1H), $4.92(\mathrm{~s}, 1 \mathrm{H}), 4.65-4.60(\mathrm{~m}, 2 \mathrm{H}), 3.77(\mathrm{~s}, 3 \mathrm{H}), 3.55-3.44(\mathrm{~m}, 1 \mathrm{H}), 3.26-3.13(\mathrm{~m}$, 2H), 2.82-2.62 (m, 2H), $1.29(\mathrm{~d}, J=6.1 \mathrm{~Hz}, 3 \mathrm{H}) .{ }^{13} \mathrm{C}$ NMR $\left(101 \mathrm{MHz}, \mathrm{CDCl}_{3}\right) \delta$ $155.7,143.6,135.9,132.1,131.4,129.4,128.2$, 127.2, 126.8, 126.3, 120.8, 119.1, 118.6, 111.6, 110.5, 68.2, 62.2, 54.9, 52.2, 49.7, 32.4, 21.1. IR (thin film): $v_{\max }\left(\mathrm{cm}^{-1}\right)$ $=3401,3058,2957,2927,2825,1745,1446,1379,1257,939,789,741,701$. HRMS-ESI calcd for $\mathrm{C}_{24} \mathrm{H}_{27} \mathrm{~N}_{2} \mathrm{O}_{3}[\mathrm{M}+\mathrm{H}]^{+}:$391.2016, Found: 391.2018.

\section{7) Synthesis of Substrate 6}

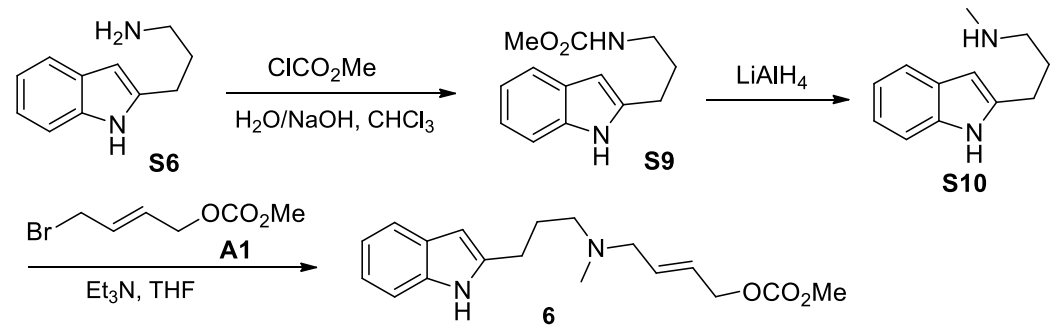

A solution of methyl chloroformate $(0.93 \mathrm{mmol}, 12 \mathrm{mmol}, 1.2$ equiv) was added dropwise to a biphasic mixture of $\mathrm{NaOH}(0.8 \mathrm{~g}, 20 \mathrm{mmol}, 2$ equiv) and $\mathbf{S 6}$ (1.74 g, 10 mmol, 1 equiv) in $\mathrm{CHCl}_{3} / \mathrm{H}_{2} \mathrm{O}(\mathrm{v} / \mathrm{v}=1 / 1,50 \mathrm{~mL})$. The reaction mixture was stirred for $16 \mathrm{~h}$ at rt. The organic phase was separated, washed with brine, dried over $\mathrm{Na}_{2} \mathrm{SO}_{4}$, filtered and concentrated by rotary evaporation. The residue was purified by silica gel column chromatography $(\mathrm{PE} / \mathrm{EA}=2 / 1)$ to afford the crude amide $\mathbf{S 9}$.

The compound $\mathbf{S 9}$ (5 mmol, 1 equiv) was added dropwise to a suspension of $\mathrm{LiAlH}_{4}(0.57 \mathrm{~g}, 15 \mathrm{mmol}, 3$ equiv $)$ in dry THF at $0{ }^{\circ} \mathrm{C}$. Then the mixture was stirred at rt for $5 \mathrm{~h}$, cooled to $0{ }^{\circ} \mathrm{C}$, and quenched with $\mathrm{H}_{2} \mathrm{O} / 10 \% \mathrm{NaOH} / \mathrm{H}_{2} \mathrm{O}(\mathrm{v} / \mathrm{v} / \mathrm{v}=1 / 2 / 3)$. The resulting mixture was filtered with celite, and the filtrate was concentrated by 
rotary evaporation to give a crude amine $\mathbf{S 1 0}$ which was used without further purification.

(E)-4-Bromobut-2-en-1-yl methyl carbonate (A1, $0.25 \mathrm{~g}, 1.2 \mathrm{mmol}, 1.2$ equiv) was added to a solution of $\mathbf{S 1 0}$ ( 1 mmol, 1 equiv) and $\mathrm{Et}_{3} \mathrm{~N}(0.42 \mathrm{ml}, 3 \mathrm{mmol}, 3$ equiv) in THF at $0{ }^{\circ} \mathrm{C}$. Then the ice bath was removed and the reaction mixture was stirred at rt until the starting material was consumed completely (monitored by TLC). The crude reaction mixture was quenched with water and extracted with EtOAc $(10 \mathrm{~mL} \times$ 3). The organic layer was washed with brine, dried over $\mathrm{Na}_{2} \mathrm{SO}_{4}$, filtered and concentrated by rotary evaporation. Then the residue was purified by $\mathrm{Al}_{2} \mathrm{O}_{3}$ column chromatography $(\mathrm{DCM} / \mathrm{MeOH}=50 / 1,20 / 1)$ to afford the desired product $\mathbf{6}$. Viscous yellow oil. ${ }^{1} \mathrm{H}$ NMR (400 MHz, $\left.\mathrm{CDCl}_{3}\right) \delta 9.24$ (s, 1H), 7.52 (d, $\left.J=7.6 \mathrm{~Hz}, 1 \mathrm{H}\right), 7.29$ $(\mathrm{d}, J=8.0 \mathrm{~Hz}, 1 \mathrm{H}), 7.14-7.00(\mathrm{~m}, 2 \mathrm{H}), 6.21(\mathrm{~s}, 1 \mathrm{H}), 5.94-5.85(\mathrm{~m}, 1 \mathrm{H}), 5.81-5.70(\mathrm{~m}$, 1H), $4.61(\mathrm{~d}, J=6.0 \mathrm{~Hz}, 2 \mathrm{H}), 3.79(\mathrm{~s}, 3 \mathrm{H}), 3.08(\mathrm{~d}, J=6.0 \mathrm{~Hz}, 2 \mathrm{H}), 2.84(\mathrm{t}, J=7.2$ $\mathrm{Hz}, 2 \mathrm{H}), 2.46(\mathrm{t}, J=6.4 \mathrm{~Hz}, 2 \mathrm{H}), 2.27(\mathrm{~s}, 3 \mathrm{H}), 1.94-1.83(\mathrm{~m}, 2 \mathrm{H}) .{ }^{13} \mathrm{C}$ NMR $(101$ $\left.\mathrm{MHz}, \mathrm{CDCl}_{3}\right) \delta 155.6,139.5,136.0,131.9,128.8,127.2,120.7,119.6,119.3,110.5$, 99.2, 67.8, 59.1, 56.8, 54.8, 41.7, 26.4, 26.3. IR (thin film): $v_{\max }\left(\mathrm{cm}^{-1}\right)=3391,3248$, 2949, 2851, 2804, 1744, 1645, 1618, 1550, 1456, 1344, 1263, 1091, 1006, 977, 941, 784, 746. HRMS-ESI calcd for $\mathrm{C}_{18} \mathrm{H}_{25} \mathrm{~N}_{2} \mathrm{O}_{3}[\mathrm{M}+\mathrm{H}]^{+}:$317.1860, Found: 317.1864 .

\section{General Procedure for Enantioselective Synthesis of Indole-Annulated} Medium-Sized Rings:
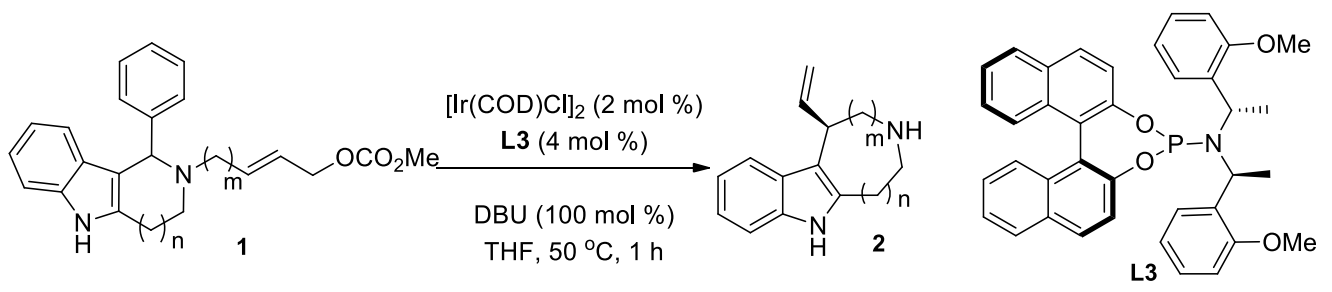

A flame dried Schlenk tube was cooled to rt and filled with argon. To this flask were added $[\mathrm{Ir}(\mathrm{COD}) \mathrm{Cl}]_{2}(5.4 \mathrm{mg}, 0.008 \mathrm{mmol}, 2 \mathrm{~mol} \%)$, phosphoramidite ligand $\mathbf{L 3}$ (9.6 mg, $0.016 \mathrm{mmol}, 4 \mathrm{~mol} \%)$, THF $(0.5 \mathrm{~mL})$ and $n$-propylamine $(0.5 \mathrm{~mL})$. The 
reaction mixture was heated at $50{ }^{\circ} \mathrm{C}$ for $30 \mathrm{~min}$ and then the volatile solvents were removed in vacuo to give a pale yellow solid. After that, allylic carbonate 1 (0.40 mmol), DBU (60 $\mu \mathrm{L}, 0.4 \mathrm{mmol})$ and THF $(4.0 \mathrm{~mL})$ were added. The reaction mixture was stirred at $50{ }^{\circ} \mathrm{C}$ for $1 \mathrm{~h}$ (the starting material was consumed completely monitored by TLC). The crude reaction mixture was quenched with brine and extracted with DCM $(10 \mathrm{~mL} \times 3)$. The organic layer was concentrated by rotary evaporation. Then the residue was purified by $\mathrm{Al}_{2} \mathrm{O}_{3}$ column chromatography (DCM/MeOH $\left.=50 / 1,20 / 1\right)$ to afford the desired products 2a-2r. The analytical data of the products are summarized below.

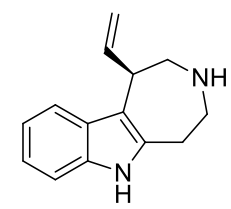

2a, yellow solid, m.p. $=80.3-85.2{ }^{\circ} \mathrm{C}, 86 \%$ yield, 98\% ee [Phenomenex Lux 5u Cellulose-4 PC-4 $(0.46 \mathrm{~cm} \times 25 \mathrm{~cm}), n$-hexane/2-propanol $=85 / 15, v=0.6 \mathrm{~mL} \cdot \mathrm{min}^{-1}$, $\lambda=230 \mathrm{~nm}, \mathrm{t}($ major $)=14.66 \mathrm{~min}, \mathrm{t}($ minor $)=24.53 \mathrm{~min}] .[\alpha]_{\mathrm{D}}{ }^{26}=+18.0(\mathrm{c}=0.25$, $\left.\mathrm{CHCl}_{3}\right) .{ }^{1} \mathrm{H} \mathrm{NMR}\left(400 \mathrm{MHz}, \mathrm{CDCl}_{3}\right) \delta 7.86(\mathrm{~s}, 1 \mathrm{H}), 7.43(\mathrm{~d}, J=7.2 \mathrm{~Hz}, 1 \mathrm{H}), 7.30(\mathrm{~d}$, $J=7.2 \mathrm{~Hz}, 1 \mathrm{H}), 7.17-7.04(\mathrm{~m}, 2 \mathrm{H}), 6.06(\mathrm{ddd}, J=17.2,10.0,5.2 \mathrm{~Hz}, 1 \mathrm{H}), 5.11(\mathrm{~d}, J$ $=10.0 \mathrm{~Hz}, 1 \mathrm{H}), 4.80(\mathrm{~d}, J=17.2 \mathrm{~Hz}, 1 \mathrm{H}), 3.85(\mathrm{~s}, 1 \mathrm{H}), 3.50-3.31(\mathrm{~m}, 2 \mathrm{H}), 3.21-3.02$ $(\mathrm{m}, 2 \mathrm{H}), 2.89(\mathrm{t}, J=12.8 \mathrm{~Hz}, 1 \mathrm{H}), 2.78(\mathrm{~d}, J=15.6 \mathrm{~Hz}, 1 \mathrm{H}) .{ }^{13} \mathrm{C}$ NMR $(101 \mathrm{MHz}$, $\left.\mathrm{CDCl}_{3}\right) \delta 138.6,135.6,134.9,129.0,121.4,119.6,117.9,116.7,112.9,110.6,53.6$, 48.0, 40.8, 31.0. IR (thin film): $v_{\max }\left(\mathrm{cm}^{-1}\right)=3392,3221,3054,2925,2852,1617$, 1491, 1458, 1335, 1265, 1088, 999, 919, 802, 741. HRMS-ESI calcd for $\mathrm{C}_{14} \mathrm{H}_{17} \mathrm{~N}_{2}$ $[\mathrm{M}+\mathrm{H}]^{+}:$213.1386, Found: 213.1389.

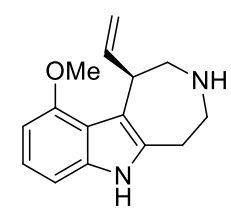

2b, viscous yellow oil, $82 \%$ yield, $98 \%$ ee [Daicel Chiralpak AD-H $(0.46 \mathrm{~cm}$ x 25 $\mathrm{cm}), n$-hexane/2-propanol/diethylamine $=80 / 20 / 0.1, v=0.7 \mathrm{~mL} \cdot \mathrm{min}^{-1}, \lambda=230 \mathrm{~nm}, \mathrm{t}$ $($ minor $)=4.44 \min , \mathrm{t}($ major $)=4.91 \mathrm{~min}] .[\alpha]_{\mathrm{D}}{ }^{28}=+18.3\left(\mathrm{c}=0.25, \mathrm{CHCl}_{3}\right) .{ }^{1} \mathrm{H} \mathrm{NMR}$ 
$\left(400 \mathrm{MHz}, \mathrm{CDCl}_{3}\right) \delta 8.28(\mathrm{~s}, 1 \mathrm{H}), 7.07-6.93(\mathrm{~m}, 1 \mathrm{H}), 6.86(\mathrm{~d}, J=8.0 \mathrm{~Hz}, 1 \mathrm{H}), 6.44$ $(\mathrm{d}, J=8.0 \mathrm{~Hz}, 1 \mathrm{H}), 6.06(\mathrm{ddd}, J=17.2,10.4,5.2 \mathrm{~Hz}, 1 \mathrm{H}), 5.10(\mathrm{~d}, J=10.4 \mathrm{~Hz}, 1 \mathrm{H})$, $4.76(\mathrm{~d}, J=17.2 \mathrm{~Hz}, 1 \mathrm{H}), 4.52(\mathrm{~s}, 1 \mathrm{H}), 3.84(\mathrm{~s}, 3 \mathrm{H}), 3.40(\mathrm{dd}, J=13.6,3.6 \mathrm{~Hz}, 1 \mathrm{H})$, $3.28(\mathrm{dt}, J=13.2,3.2 \mathrm{~Hz}, 1 \mathrm{H}), 3.09(\mathrm{dd}, J=13.6,2.4 \mathrm{~Hz}, 1 \mathrm{H}), 3.05-2.91(\mathrm{~m}, 1 \mathrm{H})$, 2.86-2.72 (m, 1H), $2.65(\mathrm{~d}, J=15.6 \mathrm{~Hz}, 1 \mathrm{H}), 2.36(\mathrm{br}, 1 \mathrm{H}) .{ }^{13} \mathrm{C}$ NMR $(101 \mathrm{MHz}$, $\left.\mathrm{CDCl}_{3}\right) \delta 154.4,140.2,136.3,135.0,121.6,118.6,115.6,113.6,104.1,99.9,55.3$, 54.2, 48.2, 42.3, 32.7. IR (thin film): $v_{\max }\left(\mathrm{cm}^{-1}\right)=3175,3068,3003,2931,2838$, 1620, 1587, 1557, 1509, 1454, 1353, 1288, 1241, 1179, 1098, 972, 908, 826, 773, 735, 674, 637. HRMS-ESI calcd for $\mathrm{C}_{15} \mathrm{H}_{19} \mathrm{~N}_{2} \mathrm{O}[\mathrm{M}+\mathrm{H}]^{+}:$243.1492, Found: 243.1493.

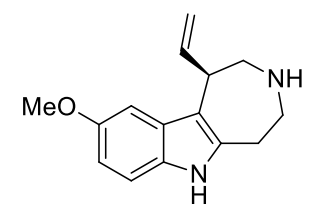

2c, viscous yellow oil, 74\% yield, 97\% ee [Daicel Chiralpak AD-H (0.46 cm x 25 $\mathrm{cm}), n$-hexane/2-propanol/diethylamine $=90 / 10 / 0.1, v=0.7 \mathrm{~mL} \cdot \mathrm{min}^{-1}, \lambda=230 \mathrm{~nm}, \mathrm{t}$ $($ minor $)=11.44 \min , \mathrm{t}$ (major) $=12.35 \mathrm{~min}] .[\alpha]_{\mathrm{D}}{ }^{24}=+11.3\left(\mathrm{c}=0.25, \mathrm{CHCl}_{3}\right) .{ }^{1} \mathrm{H}$ NMR (400 MHz, $\left.\mathrm{CDCl}_{3}\right) \delta 8.24(\mathrm{~s}, 1 \mathrm{H}), 7.14(\mathrm{~d}, J=8.8 \mathrm{~Hz}, 1 \mathrm{H}), 6.86(\mathrm{~s}, 1 \mathrm{H}), 6.76$ $(\mathrm{d}, J=8.8 \mathrm{~Hz}, 1 \mathrm{H}), 6.04(\mathrm{ddd}, J=17.2,10.4,5.6 \mathrm{~Hz}, 1 \mathrm{H}), 5.10(\mathrm{~d}, J=10.4 \mathrm{~Hz}, 1 \mathrm{H})$, $4.81(\mathrm{~d}, J=17.2 \mathrm{~Hz}, 1 \mathrm{H}), 3.91-3.68(\mathrm{~m}, 4 \mathrm{H}), 3.57-3.38(\mathrm{~m}, 2 \mathrm{H}), 3.33(\mathrm{~d}, J=13.2 \mathrm{~Hz}$, 1H), 3.14-2.96 (m, 2H), 2.94-2.80 (m, 1H), $2.75(\mathrm{~d}, J=15.6 \mathrm{~Hz}, 1 \mathrm{H}) .{ }^{13} \mathrm{C}$ NMR $(101$ $\left.\mathrm{MHz}, \mathrm{CDCl}_{3}\right) \delta 154.0,138.9,137.0,130.0,129.4,116.3,112.7,111.2,110.9,100.1$, 56.0, 53.9, 48.1, 41.3, 31.8. IR (thin film): $v_{\max }\left(\mathrm{cm}^{-1}\right)=3398,3229,3056,2929,1625$, 1586, 1482, 1454, 1321, 1262, 1209, 1145, 1112, 1028, 909, 797, 728, 643. HRMS-ESI calcd for $\mathrm{C}_{15} \mathrm{H}_{19} \mathrm{~N}_{2} \mathrm{O}[\mathrm{M}+\mathrm{H}]^{+}:$243.1492, Found: 243.1492.

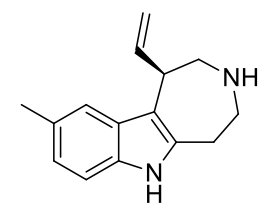

2d, pale yellow solid, m.p. $=68.2-70.4{ }^{\circ} \mathrm{C}, 72 \%$ yield, $97 \%$ ee [Daicel Chiralpak ID-3 $(0.46 \mathrm{~cm} \times 25 \mathrm{~cm}), n$-hexane/2-propanol/diethylamine $=95 / 5 / 0.1, v=0.7$ $\mathrm{mL} \cdot \mathrm{min}^{-1}, \lambda=254 \mathrm{~nm}, \mathrm{t}$ (major) $=12.18 \mathrm{~min}, \mathrm{t}($ minor $\left.)=13.91 \mathrm{~min}\right] .[\alpha]_{\mathrm{D}}{ }^{28}=+15.7$ 
$\left(\mathrm{c}=0.25, \mathrm{CHCl}_{3}\right) .{ }^{1} \mathrm{H} \mathrm{NMR}\left(400 \mathrm{MHz}, \mathrm{CDCl}_{3}\right) \delta 7.72(\mathrm{~s}, 1 \mathrm{H}), 7.21(\mathrm{~s}, 1 \mathrm{H}), 7.16(\mathrm{~d}, J$ $=8.4 \mathrm{~Hz}, 1 \mathrm{H}), 6.94(\mathrm{~d}, J=8.4 \mathrm{~Hz}, 1 \mathrm{H}), 6.05(\mathrm{ddd}, J=16.8,10.0,5.2 \mathrm{~Hz}, 1 \mathrm{H}), 5.10(\mathrm{~d}$, $J=10.0 \mathrm{~Hz}, 1 \mathrm{H}), 4.79(\mathrm{~d}, J=16.8 \mathrm{~Hz}, 1 \mathrm{H}), 3.80(\mathrm{br}, 1 \mathrm{H}), 3.47-3.28(\mathrm{~m}, 2 \mathrm{H})$, 3.13-2.98 (m, 2H), 2.91-2.80 (m, 1H), $2.71(\mathrm{~d}, J=15.6 \mathrm{~Hz}, 1 \mathrm{H}), 2.43$ (s, 3H), 1.86 (br, 1H). ${ }^{13} \mathrm{C} \mathrm{NMR}\left(101 \mathrm{MHz}, \mathrm{CDCl}_{3}\right) \delta 139.4,136.5,133.1,129.4,128.6,122.6,117.5$, 116.0, 112.8, 110.1, 54.4, 48.5, 41.7, 32.8, 21.7. IR (thin film): $v_{\max }\left(\mathrm{cm}^{-1}\right)=3396$, 3006, 2918, 1630, 1588, 1456, 1319, 1260, 1217, 1091, 1018, 917, 865, 795, 750, 701, 664. HRMS-ESI calcd for $\mathrm{C}_{15} \mathrm{H}_{19} \mathrm{~N}_{2}[\mathrm{M}+\mathrm{H}]^{+}$: 227.1543, Found: 227.1543 .<smiles>C=CC1CNCCc2[nH]c3ccc(Cl)cc3c21</smiles>

2e, pale yellow solid, m.p. $=72.6-75.3{ }^{\circ} \mathrm{C}, 76 \%$ yield, $97 \%$ ee $[$ Daicel Chiralpak AD-H $(0.46 \mathrm{~cm} \times 25 \mathrm{~cm}), n$-hexane/2-propanol/diethylamine $=95 / 5 / 0.1, v=0.7$ $\mathrm{mL} \cdot \min ^{-1}, \lambda=230 \mathrm{~nm}, \mathrm{t}($ minor $)=19.07 \mathrm{~min}, \mathrm{t}($ major $\left.)=20.33 \mathrm{~min}\right] .[\alpha]_{\mathrm{D}}{ }^{26}=+17.5$ $\left(\mathrm{c}=0.25, \mathrm{CHCl}_{3}\right) .{ }^{1} \mathrm{H} \mathrm{NMR}\left(400 \mathrm{MHz}, \mathrm{CDCl}_{3}\right) \delta 8.39(\mathrm{~s}, 1 \mathrm{H}), 7.37(\mathrm{~s}, 1 \mathrm{H}), 7.15(\mathrm{~d}, J$ $=8.4 \mathrm{~Hz}, 1 \mathrm{H}), 7.02(\mathrm{dd}, J=8.4,1.6 \mathrm{~Hz}, 1 \mathrm{H}), 6.01(\mathrm{ddd}, J=16.8,10.0,5.6 \mathrm{~Hz}, 1 \mathrm{H})$, $5.09(\mathrm{~d}, J=10.0 \mathrm{~Hz}, 1 \mathrm{H}), 4.75$ (d, $J=16.8 \mathrm{~Hz}, 1 \mathrm{H}), 3.72(\mathrm{~s}, 1 \mathrm{H}), 3.40(\mathrm{dd}, J=13.2$, $3.2 \mathrm{~Hz}, 1 \mathrm{H}), 3.36-3.25$ (m, 1H), 3.10-2.95 (m, 2H), 2.89-2.70 (m, 2H), 2.18 (br, 1H). ${ }^{13} \mathrm{C}$ NMR $\left(101 \mathrm{MHz}, \mathrm{CDCl}_{3}\right) \delta 139.1,138.0,133.2,130.3,125.0,121.2,117.3,116.1$, 113.0, 111.4, 54.4, 48.4, 41.8, 32.7. IR (thin film): $v_{\max }\left(\mathrm{cm}^{-1}\right)=3407,3167,3036$, 2924, 2857, 1618, 1574, 1449, 1378, 1320, 1294, 1253, 1226, 1184, 1058, 968, 919, 859, 795, 757, 714. HRMS-ESI calcd for $\mathrm{C}_{14} \mathrm{H}_{16} \mathrm{ClN}_{2}[\mathrm{M}+\mathrm{H}]^{+}$: 247.0997, Found: 247.0998 .

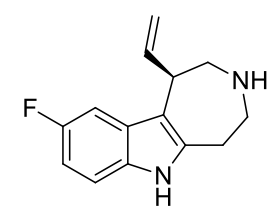

2f, pale yellow solid, m.p. $=114.3-119.6{ }^{\circ} \mathrm{C}, 64 \%$ yield, $96 \%$ ee [Daicel Chiralpak ID-3 $(0.46 \mathrm{~cm} \times 25 \mathrm{~cm}), n$-hexane/2-propanol $/$ diethylamine $=95 / 5 / 0.1, v=$ $0.7 \mathrm{~mL} \cdot \min ^{-1}, \lambda=230 \mathrm{~nm}, \mathrm{t}($ minor $)=13.27 \mathrm{~min}, \mathrm{t}($ major $\left.)=14.13 \mathrm{~min}\right] \cdot[\alpha]_{\mathrm{D}}^{26}=$ 
$+10.8\left(\mathrm{c}=0.25, \mathrm{CHCl}_{3}\right) .{ }^{1} \mathrm{H} \mathrm{NMR}\left(400 \mathrm{MHz}, \mathrm{CDCl}_{3}\right) \delta 8.03(\mathrm{~s}, 1 \mathrm{H}), 7.17(\mathrm{dd}, J=8.8$, $4.4 \mathrm{~Hz}, 1 \mathrm{H}), 7.06(\mathrm{dd}, J=9.6,2.0 \mathrm{~Hz}, 1 \mathrm{H}), 6.84(\mathrm{td}, J=9.2,2.4 \mathrm{~Hz}, 1 \mathrm{H}), 6.04$ (ddd, $J=17.2,10.0,5.6 \mathrm{~Hz}, 1 \mathrm{H}), 5.10(\mathrm{~d}, J=10.0 \mathrm{~Hz}, 1 \mathrm{H}), 4.78(\mathrm{~d}, J=17.2 \mathrm{~Hz}, 1 \mathrm{H})$, 3.75-3.68 (m, 1H), 3.47-3.28 (m, 2H), 3.13-3.00 (m, 2H), 2.92-2.82 (m, 1H), 2.79-2.71 (m, 1H), 2.04 (br, 1H). ${ }^{19} \mathrm{~F}$ NMR $\left(376 \mathrm{MHz}, \mathrm{CDCl}_{3}\right) \delta-125.0(\mathrm{~m}) .{ }^{13} \mathrm{C} \mathrm{NMR}$ $\left(101 \mathrm{MHz}, \mathrm{CDCl}_{3}\right) \delta 157.9(\mathrm{~d}, J=233.7 \mathrm{~Hz}), 139.1,138.2,131.2,129.6(\mathrm{~d}, J=9.6$ $\mathrm{Hz}), 115.8,113.5,110.8(\mathrm{~d}, J=9.7 \mathrm{~Hz}), 109.1(\mathrm{~d}, J=26.2 \mathrm{~Hz}), 102.8(\mathrm{~d}, J=23.5$ Hz), 54.4, 48.4, 41.9, 32.8. IR (thin film): $v_{\max }\left(\mathrm{cm}^{-1}\right)=3408,3178,3151,3046,2924$, 2871, 2726, 1629, 1582, 1486, 1452, 1376, 1326, 1257, 1220, 1181, 1133, 1082, 981, 921, 844, 791, 753. HRMS-ESI calcd for $\mathrm{C}_{14} \mathrm{H}_{16} \mathrm{FN}_{2}[\mathrm{M}+\mathrm{H}]^{+}$: 231.1292, Found: 231.1295 .

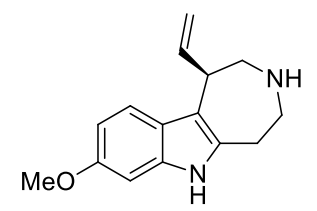

2g, pale yellow solid, m.p. $=66.4-70.5{ }^{\circ} \mathrm{C}, 79 \%$ yield, 98\% ee [Phenomenex Lux 5u Cellulose-4 PC-4 $(0.46 \mathrm{~cm}$ x $25 \mathrm{~cm}), n$-hexane/2-propanol $=85 / 15, v=1.0$ $\mathrm{mL} \cdot \min ^{-1}, \lambda=230 \mathrm{~nm}, \mathrm{t}$ (major) $=14.19 \mathrm{~min}, \mathrm{t}($ minor $\left.)=40.83 \mathrm{~min}\right] .[\alpha]_{\mathrm{D}}{ }^{24}=+8.7(\mathrm{c}$ $\left.=0.25, \mathrm{CHCl}_{3}\right) .{ }^{1} \mathrm{H} \mathrm{NMR}\left(400 \mathrm{MHz}, \mathrm{CDCl}_{3}\right) \delta 7.94(\mathrm{~s}, 1 \mathrm{H}), 7.29(\mathrm{~d}, J=8.4 \mathrm{~Hz}, 1 \mathrm{H})$, 6.83-6.69 (m, 2H), 6.03 (ddd, $J=16.8,10.0,5.6 \mathrm{~Hz}, 1 \mathrm{H}), 5.08(\mathrm{~d}, J=10.0 \mathrm{~Hz}, 1 \mathrm{H})$, $4.79(\mathrm{dt}, J=16.8,1.6 \mathrm{~Hz}, 1 \mathrm{H}), 3.81(\mathrm{~s}, 3 \mathrm{H}), 3.79-3.72(\mathrm{~m}, 1 \mathrm{H}), 3.40$ (dd, $J=13.6$, $3.2 \mathrm{~Hz}, 1 \mathrm{H}), 3.31(\mathrm{dt}, J=13.2,3.6 \mathrm{~Hz}, 1 \mathrm{H}), 3.09(\mathrm{dd}, J=13.6,2.8 \mathrm{~Hz}, 1 \mathrm{H})$, 3.06-2.94 (m, 1H), 2.90-2.79 (m, 1H), 2.71-2.64 (m, 1H), $2.18(\mathrm{br}, 1 \mathrm{H}) .{ }^{13} \mathrm{C}$ NMR $\left(101 \mathrm{MHz}, \mathrm{CDCl}_{3}\right) \delta 155.9,139.5,135.5,134.9,123.7,118.4,115.8,113.1,108.8$, 94.6, 55.9, 54.5, 48.6, 41.9, 32.6. IR (thin film): $v_{\max }\left(\mathrm{cm}^{-1}\right)=3398,3074,2998,2926$, 2833, 1627, 1568, 1499, 1462, 1340, 1292, 1264, 1225, 1199, 1157, 1112, 1028, 911, 803, 750, 664. HRMS-ESI calcd for $\mathrm{C}_{15} \mathrm{H}_{19} \mathrm{~N}_{2} \mathrm{O}[\mathrm{M}+\mathrm{H}]^{+}$: 243.1492, Found: 243.1491. 


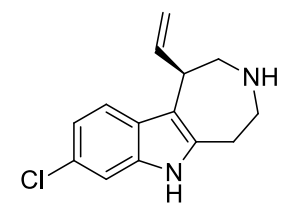

2h, yellow solid, m.p. $=67.8-72.9{ }^{\circ} \mathrm{C}, 89 \%$ yield, 98\% ee $[$ Phenomenex Lux 5u Cellulose-4 PC-4 $(0.46 \mathrm{~cm} \times 25 \mathrm{~cm}), n$-hexane/2-propanol $=85 / 15, v=1.0 \mathrm{~mL} \cdot \mathrm{min}^{-1}$, $\lambda=230 \mathrm{~nm}, \mathrm{t}$ (major) $=8.55 \mathrm{~min}, \mathrm{t}($ minor $)=25.09 \mathrm{~min}] .[\alpha]_{\mathrm{D}}^{23}=+13.6(\mathrm{c}=0.25$, $\left.\mathrm{CHCl}_{3}\right) .{ }^{1} \mathrm{H}$ NMR $\left(400 \mathrm{MHz}, \mathrm{CDCl}_{3}\right) \delta 8.21(\mathrm{~s}, 1 \mathrm{H}), 7.43-7.15(\mathrm{~m}, 3 \mathrm{H}), 7.02(\mathrm{~d}, J=$ $8.4 \mathrm{~Hz}, 1 \mathrm{H}), 6.02$ (ddd, $J=17.2,10.0,6.0 \mathrm{~Hz}, 1 \mathrm{H}), 5.09$ (d, $J=10.0 \mathrm{~Hz}, 1 \mathrm{H}), 4.75$ (d, $J=17.2 \mathrm{~Hz}, 1 \mathrm{H}), 3.77(\mathrm{~s}, 1 \mathrm{H}), 3.51-3.22(\mathrm{~m}, 2 \mathrm{H}), 3.15-2.95(\mathrm{~m}, 2 \mathrm{H}), 2.86(\mathrm{t}, J=12.8$ $\mathrm{Hz}, 1 \mathrm{H}), 2.73(\mathrm{~d}, J=15.6 \mathrm{~Hz}, 1 \mathrm{H}), 2.35(\mathrm{br}, 1 \mathrm{H}) .{ }^{13} \mathrm{C} \mathrm{NMR}\left(101 \mathrm{MHz}, \mathrm{CDCl}_{3}\right) \delta$ 139.1, 137.0, 135.2, 127.8, 126.9, 119.9, 118.7, 116.1, 113.4, 110.4, 54.3, 48.4, 41.7, 32.5. IR (thin film): $v_{\max }\left(\mathrm{cm}^{-1}\right)=3406,3174,3057,2921,2851,2718,1678,1617$, 1556, 1462, 1420, 1332, 1222, 1163, 1058, 991, 894, 841, 801, 749. HRMS-ESI calcd for $\mathrm{C}_{14} \mathrm{H}_{16} \mathrm{ClN}_{2}[\mathrm{M}+\mathrm{H}]^{+}:$247.0997, Found: 247.0999.

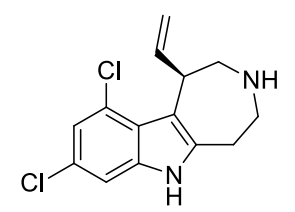

2i, viscous yellow oil, 47\% yield, 98\% ee [Phenomenex Lux 5u Cellulose-4 PC-4 $(0.46 \mathrm{~cm} \times 25 \mathrm{~cm}), n$-hexane $/ 2$-propanol $=85 / 15, v=1.0 \mathrm{~mL} \cdot \mathrm{min}^{-1}, \lambda=230 \mathrm{~nm}, \mathrm{t}$ $($ major $)=6.22 \min , \mathrm{t}($ minor $)=13.56 \mathrm{~min}] .[\alpha]_{\mathrm{D}}{ }^{28}=+15.4\left(\mathrm{c}=0.25, \mathrm{CHCl}_{3}\right) .{ }^{1} \mathrm{H}$ NMR $\left(400 \mathrm{MHz}, \mathrm{CDCl}_{3}\right) \delta 8.48(\mathrm{~s}, 1 \mathrm{H}), 7.16(\mathrm{~d}, J=1.2 \mathrm{~Hz}, 1 \mathrm{H}), 7.01(\mathrm{~d}, J=1.2 \mathrm{~Hz}$, 1H), 6.06 (ddd, $J=16.8,10.0,4.8 \mathrm{~Hz} 1 \mathrm{H}), 5.17(\mathrm{~d}, J=10.0 \mathrm{~Hz}, 1 \mathrm{H}), 4.82-4.57(\mathrm{~m}$, 2H), $3.44(\mathrm{dd}, J=13.6,3.6 \mathrm{~Hz}, 1 \mathrm{H}), 3.30(\mathrm{dt}, J=12.8,3.2 \mathrm{~Hz}, 1 \mathrm{H}), 3.14-2.99(\mathrm{~m}$, 2H), 2.88-2.70 (m, 2H), 2.04 (br, 1H). $\left.{ }^{13} \mathrm{C} \mathrm{NMR} \mathrm{(101} \mathrm{MHz,} \mathrm{CDCl}_{3}\right) \delta$ 139.7, 138.8, $136.2,126.4,125.8,124.1,121.1,116.5,114.0,109.4,54.1,47.8,41.5,32.9$. IR (thin film): $v_{\max }\left(\mathrm{cm}^{-1}\right)=3154,3080,2929,2854,1641,1614,1548,1451,1414,1328$, 1296, 1226, 1186, 1147, 1105, 1081, 975, 916, 833, 797, 734, 699. HRMS-ESI calcd for $\mathrm{C}_{14} \mathrm{H}_{15} \mathrm{Cl}_{2} \mathrm{~N}_{2}[\mathrm{M}+\mathrm{H}]^{+}$: 281.0607, Found: 281.0605 . 


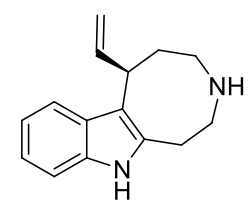

2j, viscous yellow oil, 57\% yield, 94\% ee [Daicel Chiralpak AD-H (0.46 cm x 25 $\mathrm{cm}), n$-hexane/2-propanol/diethylamine $=90 / 10 / 0.1, v=0.7 \mathrm{~mL} \cdot \mathrm{min}^{-1}, \lambda=230 \mathrm{~nm}, \mathrm{t}$ $($ major $)=7.28 \mathrm{~min}, \mathrm{t}($ minor $)=7.99 \mathrm{~min}] .[\alpha]_{\mathrm{D}}{ }^{27}=+9.1\left(\mathrm{c}=0.25, \mathrm{CHCl}_{3}\right) .{ }^{1} \mathrm{H} \mathrm{NMR}$ $\left(400 \mathrm{MHz}, \mathrm{CDCl}_{3}\right) \delta 8.39(\mathrm{~s}, 1 \mathrm{H}), 7.60(\mathrm{~d}, J=8.0 \mathrm{~Hz}, 1 \mathrm{H}), 7.27(\mathrm{~d}, J=8.0 \mathrm{~Hz}, 1 \mathrm{H})$, 7.13-6.94 (m, 2H), $6.29(\mathrm{ddd}, J=18.4,11.2,5.6 \mathrm{~Hz}, 1 \mathrm{H}), 5.27-5.09(\mathrm{~m}, 2 \mathrm{H})$, 4.01-3.86 (m, 1H), 3.13-2.71 (m, 5H), 2.48-2.34 (m, 1H), 2.06-1.86 (m, 2H). ${ }^{13} \mathrm{C}$ NMR $\left(101 \mathrm{MHz}, \mathrm{CDCl}_{3}\right) \delta 141.9,135.4,134.0,128.0,120.8,119.6,118.9,113.8$, 113.1, 110.7, 49.7, 47.2, 37.6, 36.2, 30.3. IR (thin film): $v_{\max }\left(\mathrm{cm}^{-1}\right)=3363,2958$, 2924, 2854, 1638, 1457, 1333, 1260, 1089, 914, 800, 741. HRMS-ESI calcd for $\mathrm{C}_{15} \mathrm{H}_{19} \mathrm{~N}_{2}[\mathrm{M}+\mathrm{H}]^{+}:$227.1543, Found: 227.1542.

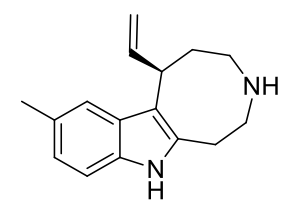

2k, viscous yellow oil, 59\% yield, $97 \%$ ee [Daicel Chiralpak IC (0.46 cm x 25 $\mathrm{cm}), n$-hexane/2-propanol/diethylamine $=95 / 5 / 0.1, v=0.7 \mathrm{~mL} \cdot \mathrm{min}^{-1}, \lambda=230 \mathrm{~nm}, \mathrm{t}$ $($ major $)=22.81 \mathrm{~min}, \mathrm{t}($ minor $)=27.88 \mathrm{~min},] .[\alpha]_{\mathrm{D}}{ }^{26}=+6.0\left(\mathrm{c}=0.25, \mathrm{CHCl}_{3}\right) .{ }^{1} \mathrm{H}$ NMR (400 MHz, $\left.\mathrm{CDCl}_{3}\right) \delta 8.19(\mathrm{~s}, 1 \mathrm{H}), 7.38(\mathrm{~s}, 1 \mathrm{H}), 7.17$ (d, J=8.0 Hz, 1H), 6.91 (d, $J=8.0 \mathrm{~Hz}, 1 \mathrm{H}), 6.31$ (ddd, $J=17.2,10.8,5.2 \mathrm{~Hz}, 1 \mathrm{H}), 5.24-5.11(\mathrm{~m}, 2 \mathrm{H}), 3.96-3.87$ (m, 1H), 3.11-2.97 (m, 2H), 2.96-2.83 (m, 2H), 2.82-2.72 (m, 1H), 2.45-2.32 (m, 4H), 2.07-1.86 (m, 3H). ${ }^{13} \mathrm{C}$ NMR (101 MHz, $\left.\mathrm{CDCl}_{3}\right) \delta 141.9,134.0,133.7,128.2,128.1$, $122.5,119.2,113.9,112.7,110.4,49.7,47.2,37.5,36.1,30.1,21.7$. IR (thin film): $v_{\max }\left(\mathrm{cm}^{-1}\right)=3390,3191,3034,2918,2856,1678,1637,1582,1457,1348,1321$, 1259, 1173, 1096, 1016, 997, 909, 864, 795, 753, 731, 661. HRMS-ESI calcd for $\mathrm{C}_{16} \mathrm{H}_{21} \mathrm{~N}_{2}[\mathrm{M}+\mathrm{H}]^{+}:$241.1699, Found: 241.1696. 
<smiles>C=CC1CCNCCc2[nH]c3ccc(Cl)cc3c21</smiles>

2l, pale yellow solid, m.p. $=72.5-76.7^{\circ} \mathrm{C}, 64 \%$ yield, $93 \%$ ee [Daicel Chiralpak AD-H $(0.46 \mathrm{~cm} \times 25 \mathrm{~cm}), n$-hexane/2-propanol/diethylamine $=95 / 5 / 0.1, v=0.7$ $\mathrm{mL} \cdot \mathrm{min}^{-1}, \lambda=230 \mathrm{~nm}, \mathrm{t}($ minor $)=13.58 \mathrm{~min}, \mathrm{t}($ major $\left.)=14.67 \mathrm{~min}\right] .[\alpha]_{\mathrm{D}}{ }^{26}=+8.6(\mathrm{c}$ $\left.=0.25, \mathrm{CHCl}_{3}\right) .{ }^{1} \mathrm{H}$ NMR $\left(400 \mathrm{MHz}, \mathrm{CDCl}_{3}\right) \delta 8.74(\mathrm{~s}, 1 \mathrm{H}), 7.55(\mathrm{~s}, 1 \mathrm{H}), 7.20(\mathrm{~d}, J=$ $8.8 \mathrm{~Hz}, 1 \mathrm{H}), 7.00(\mathrm{~d}, J=8.8 \mathrm{~Hz}, 1 \mathrm{H}), 6.26(\mathrm{ddd}, J=16.8,10.8,5.2 \mathrm{~Hz} 1 \mathrm{H})$, 5.35-5.07 (m, 2H), 3.99-3.82 (m, 1H), 3.43-3.19 (m, 1H), 3.14-2.87 (m, 3H), 2.83-2.72 (m, 1H), 2.38-2.24 (m, 1H), 2.08-1.93 (m, 1H), 1.93-1.79 (m, 1H). ${ }^{13} \mathrm{C}$ NMR $\left(101 \mathrm{MHz}, \mathrm{CDCl}_{3}\right) \delta 141.3,135.9,133.8,129.0,124.5,120.9,118.9,114.3$, 112.7, 111.8, 49.9, 47.1, 37.2, 36.0, 30.0. IR (thin film): $v_{\max }\left(\mathrm{cm}^{-1}\right)=3398,3172$, 3079, 3034, 2919, 2853, 2747, 1641, 1570, 1468, 1444, 1352, 1319, 1261, 1182, 1112 , 1057, 1003, 914, 860, 796, 738, 686. HRMS-ESI calcd for $\mathrm{C}_{15} \mathrm{H}_{18} \mathrm{ClN}_{2}[\mathrm{M}+\mathrm{H}]^{+}$: 261.1153, Found: 261.1156.

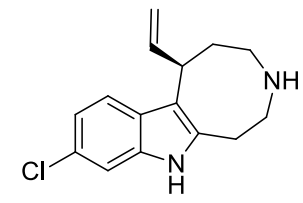

2m, viscous yellow oil, 64\% yield, 98\% ee [Daicel Chiralpak ID-3 (0.46 cm x 25 $\mathrm{cm}), n$-hexane/2-propanol/diethylamine $=97 / 3 / 0.1, v=0.7 \mathrm{~mL} \cdot \mathrm{min}^{-1}, \lambda=240 \mathrm{~nm}, \mathrm{t}$ $($ major $)=13.68 \mathrm{~min}, \mathrm{t}($ minor $)=16.14 \mathrm{~min}] .[\alpha]_{\mathrm{D}}{ }^{26}=-6.0\left(\mathrm{c}=0.25, \mathrm{CHCl}_{3}\right) .{ }^{1} \mathrm{H} \mathrm{NMR}$ $\left(400 \mathrm{MHz}, \mathrm{CDCl}_{3}\right) \delta 8.23(\mathrm{~s}, 1 \mathrm{H}), 7.50(\mathrm{~d}, J=8.4 \mathrm{~Hz}, 1 \mathrm{H}), 7.23(\mathrm{~d}, J=2.0 \mathrm{~Hz}, 1 \mathrm{H})$, $6.98(\mathrm{dd}, J=8.4,2.0 \mathrm{~Hz}, 1 \mathrm{H}), 6.27$ (ddd, $J=16.8,10.8,5.6 \mathrm{~Hz} 1 \mathrm{H}), 5.25-5.12(\mathrm{~m}$, 2H), 3.98-3.87 (m, 1H), 3.15-2.72 (m, 5H), 2.41-2.28 (m, 1H), 2.08-1.84 (m, 2H), 1.80 (br, 1H). ${ }^{13} \mathrm{C}$ NMR (101 MHz, $\left.\mathrm{CDCl}_{3}\right) \delta 141.6,135.8,134.9,126.7,126.6,120.5$, 119.6, 114.1, 113.3, 110.6, 49.8, 47.2, 37.3, 36.1, 30.3. IR (thin film): $v_{\max }\left(\mathrm{cm}^{-1}\right)=$ 3311, 3141, 3085, 2919, 2852, 1681, 1641, 1557, 1459, 1322, 1239, 1178, 1119, 1061, 1007, 914, 875, 806, 728. HRMS-ESI calcd for $\mathrm{C}_{15} \mathrm{H}_{18} \mathrm{ClN}_{2}[\mathrm{M}+\mathrm{H}]^{+}:$261.1153, Found: 261.1152 . 


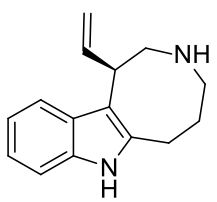

2n, viscous yellow oil, 75\% yield, 98\% ee [Daicel Chiralpak ID-3 (0.46 cm x 25 $\mathrm{cm}), n$-hexane/2-propanol $/$ diethylamine $=90 / 10 / 0.1, v=0.7 \mathrm{~mL} \cdot \mathrm{min}^{-1}, \lambda=230 \mathrm{~nm}, \mathrm{t}$ $($ major $)=9.19 \mathrm{~min}, \mathrm{t}($ minor $)=10.97 \mathrm{~min}] .[\alpha]_{\mathrm{D}}{ }^{28}=+14.2\left(\mathrm{c}=0.25, \mathrm{CHCl}_{3}\right) .{ }^{1} \mathrm{H} \mathrm{NMR}$ $\left(400 \mathrm{MHz}, \mathrm{CDCl}_{3}\right) \delta 8.41(\mathrm{~s}, 1 \mathrm{H}), 7.58(\mathrm{~d}, J=7.6 \mathrm{~Hz}, 1 \mathrm{H}), 7.25(\mathrm{~d}, J=8.0 \mathrm{~Hz}, 1 \mathrm{H})$, 7.14-6.97 (m, 2H), 6.33 (ddd, $J=16.8,11.2,5.6 \mathrm{~Hz}, 1 \mathrm{H}), 5.32-5.15(\mathrm{~m}, 2 \mathrm{H})$, 3.91-3.75 (m, 1H), 3.17-2.97 (m, 3H), 2.96-2.74 (m, 2H), 2.61-2.52 (m, 1H), $2.12(\mathrm{br}$, $1 \mathrm{H}), 1.91-1.76(\mathrm{~m}, 1 \mathrm{H}), 1.71-1.49(\mathrm{~m}, 1 \mathrm{H}) .{ }^{13} \mathrm{C} \mathrm{NMR}\left(101 \mathrm{MHz}, \mathrm{CDCl}_{3}\right) \delta 139.4$, 136.7, 135.4, 127.8, 120.8, 119.11, 119.06, 115.2, 110.8, 54.2, 47.9, 40.5, 32.4, 25.7. IR (thin film): $v_{\max }\left(\mathrm{cm}^{-1}\right)=3394,3180,3053,2923,2854,2705,1614,1487,1458$, 1341, 1294, 1262, 1215, 1105, 1014, 914, 828, 738, 663. HRMS-ESI calcd for $\mathrm{C}_{15} \mathrm{H}_{19} \mathrm{~N}_{2}[\mathrm{M}+\mathrm{H}]^{+}:$227.1543, Found: 227.1543.

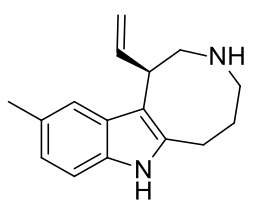

2o, viscous yellow oil, $84 \%$ yield, $98 \%$ ee [Daicel Chiralpak AD-H $(0.46 \mathrm{~cm}$ x 25 $\mathrm{cm}), n$-hexane/2-propanol/diethylamine $=96 / 4 / 0.1, v=0.7 \mathrm{~mL} \cdot \min ^{-1}, \lambda=230 \mathrm{~nm}, \mathrm{t}$ $($ major $)=13.91 \mathrm{~min}, \mathrm{t}($ minor $)=15.96 \mathrm{~min}] .[\alpha]_{\mathrm{D}}{ }^{26}=+51.8\left(\mathrm{c}=0.25, \mathrm{CHCl}_{3}\right) .{ }^{1} \mathrm{H}$ NMR (400 MHz, $\left.\mathrm{CDCl}_{3}\right) \delta 8.21(\mathrm{~s}, 1 \mathrm{H}), 7.37(\mathrm{~s}, 1 \mathrm{H}), 7.16(\mathrm{~d}, J=8.0 \mathrm{~Hz}, 1 \mathrm{H}), 6.92$ $(\mathrm{d}, J=8.0 \mathrm{~Hz}, 1 \mathrm{H}), 6.34(\mathrm{ddd}, J=17.6,10.0,5.6 \mathrm{~Hz}, 1 \mathrm{H}), 5.33-5.12(\mathrm{~m}, 2 \mathrm{H})$, 3.87-3.77 (m, 1H), 3.14-2.98 (m, 3H), 2.96-2.86 (m, 1H), 2.84-2.72 (m, 1H), 2.59-2.50 (m, 1H), $2.41(\mathrm{~s}, 3 \mathrm{H}), 1.88-1.78(\mathrm{~m}, 1 \mathrm{H}), 1.68-1.60(\mathrm{~m}, 1 \mathrm{H}) .{ }^{13} \mathrm{C}$ NMR $(101$ $\left.\mathrm{MHz}, \mathrm{CDCl}_{3}\right) \delta 139.6,136.9,133.7,128.2,128.0,122.3,118.8,115.1,110.5,110.4$, 54.5, 48.1, 40.7, 32.7, 25.8, 21.7. IR (thin film): $v_{\max }\left(\mathrm{cm}^{-1}\right)=3390,3185,3008,2917$, 2856, 1633, 1605, 1584, 1458, 1322, 1258, 1215, 1110, 1028, 997, 912, 794, 750, 663. HRMS-ESI calcd for $\mathrm{C}_{16} \mathrm{H}_{21} \mathrm{~N}_{2}[\mathrm{M}+\mathrm{H}]^{+}:$241.1699, Found: 241.1703 . 


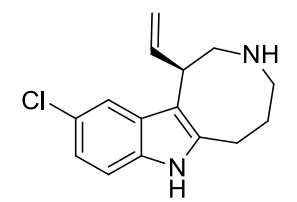

2p, viscous yellow oil, $80 \%$ yield, 98\% ee [Daicel Chiralpak AD-H (0.46 cm x 25 $\mathrm{cm}), n$-hexane/2-propanol/diethylamine $=90 / 10 / 0.1, v=0.7 \mathrm{~mL} \cdot \mathrm{min}^{-1}, \lambda=230 \mathrm{~nm}, \mathrm{t}$ $($ major $)=7.43 \mathrm{~min}, \mathrm{t}($ minor $)=8.09 \mathrm{~min}] .[\alpha]_{\mathrm{D}}{ }^{26}=+37.4\left(\mathrm{c}=0.25, \mathrm{CHCl}_{3}\right) .{ }^{1} \mathrm{H} \mathrm{NMR}$ $\left(400 \mathrm{MHz}, \mathrm{CDCl}_{3}\right) \delta 8.15(\mathrm{~s}, 1 \mathrm{H}), 7.54(\mathrm{~d}, J=2.0 \mathrm{~Hz}, 1 \mathrm{H}), 7.17(\mathrm{~d}, J=8.4 \mathrm{~Hz}, 1 \mathrm{H})$, $7.04(\mathrm{dd}, J=8.4,2.0 \mathrm{~Hz}, 1 \mathrm{H}), 6.35-6.29(\mathrm{ddd}, J=17.2,10.8,5.6 \mathrm{~Hz} 1 \mathrm{H}), 5.28-5.16$ (m, 2H), 3.83-3.74 (m, 1H), 3.16-2.90 (m, 4H), 2.86-2.77 (m, 1H), 2.61-2.48 (m, 1H), 1.91-1.78 (m, 1H), 1.74-1.61 (m, 1H), $\left.1.52(\mathrm{br}, 1 \mathrm{H}) .{ }^{13} \mathrm{C} \mathrm{NMR} \mathrm{(101} \mathrm{MHz,} \mathrm{CDCl}_{3}\right) \delta$ 138.3, 138.0, 133.8, 128.7, 124.8, 121.1, 118.5, 115.9, 111.8, 110.2, 53.5, 47.4, 39.5, 31.2, 25.6. IR (thin film): $v_{\max }\left(\mathrm{cm}^{-1}\right)=3402,3172,3080,2922,2852,2731,1635$, 1605, 1570, 1443, 1355, 1299, 1260, 1106, 1058, 1000, 908, 859, 797, 728. HRMS-ESI calcd for $\mathrm{C}_{15} \mathrm{H}_{18} \mathrm{ClN}_{2}[\mathrm{M}+\mathrm{H}]^{+}:$261.1153, Found: 261.1153.

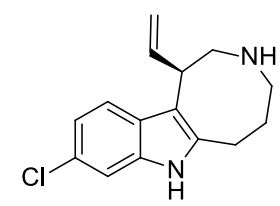

2q, viscous yellow oil, 83\% yield, 99\% ee [Daicel Chiralcel OD-H $(0.46 \mathrm{~cm} \times 25$ $\mathrm{cm}), n$-hexane/2-propanol/diethylamine $=96 / 4 / 0.1, v=0.7 \mathrm{~mL} \cdot \min ^{-1}, \lambda=230 \mathrm{~nm}, \mathrm{t}$ $($ minor $)=15.40 \mathrm{~min}, \mathrm{t}($ major $)=19.25 \mathrm{~min}] .[\alpha]_{\mathrm{D}}{ }^{26}=+30.1\left(\mathrm{c}=0.25, \mathrm{CHCl}_{3}\right) .{ }^{1} \mathrm{H}$ NMR $\left(400 \mathrm{MHz}, \mathrm{CDCl}_{3}\right) \delta 8.17(\mathrm{~s}, 1 \mathrm{H}), 7.47(\mathrm{~d}, J=8.4 \mathrm{~Hz}, 1 \mathrm{H}), 7.25(\mathrm{~d}, J=1.6 \mathrm{~Hz}$, 1H), 6.99 (dd, $J=8.4,1.6 \mathrm{~Hz}, 1 \mathrm{H}), 6.28(\mathrm{ddd}, J=17.2,10.4,5.6 \mathrm{~Hz}, 1 \mathrm{H}), 5.25-5.16$ $(\mathrm{m}, 2 \mathrm{H}), 3.88-3.73(\mathrm{~m}, 1 \mathrm{H}), 3.12(\mathrm{dd}, J=13.6,4.8 \mathrm{~Hz}, 1 \mathrm{H}), 3.08-3.00(\mathrm{~m}, 2 \mathrm{H})$, $3.00-2.90(\mathrm{~m}, 1 \mathrm{H}), 2.86-2.77(\mathrm{~m}, 1 \mathrm{H}), 2.62-2.50(\mathrm{~m}, 1 \mathrm{H}), 1.91-1.78(\mathrm{~m}, 1 \mathrm{H})$, 1.74-1.63 (m, 1H), $1.54(\mathrm{br}, 1 \mathrm{H}) .{ }^{13} \mathrm{C} \mathrm{NMR}\left(101 \mathrm{MHz}, \mathrm{CDCl}_{3}\right) \delta$ 139.3, 137.4, 135.7, $126.7,126.6,119.9,119.8,115.4,111.4,110.6,54.5,48.1,40.8,32.7,25.7$. IR (thin film): $v_{\max }\left(\mathrm{cm}^{-1}\right)=3400,3165,3080,2919,2852,1636,1613,1553,1461,1429$, 1319, 1230, 1109, 1062, 996, 919, 844, 802, 744, 616. HRMS-ESI calcd for $\mathrm{C}_{15} \mathrm{H}_{18} \mathrm{ClN}_{2}[\mathrm{M}+\mathrm{H}]^{+}:$261.1153, Found: 261.1155. 


\section{General Procedure for Enantioselective Synthesis of Indole-Annulated}

Nine-Membered Rings:

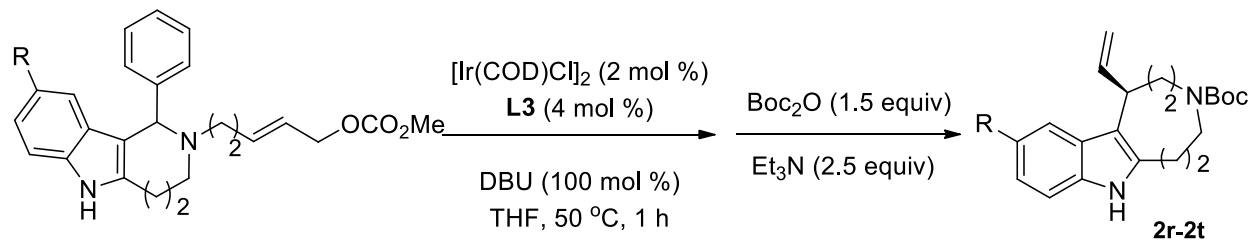

A flame dried Schlenk tube was cooled to rt and filled with argon. To this flask were added $[\mathrm{Ir}(\mathrm{COD}) \mathrm{Cl}]_{2}(5.4 \mathrm{mg}, 0.008 \mathrm{mmol}, 2 \mathrm{~mol} \%)$, phosphoramidite ligand $\mathbf{L 3}$ (9.6 mg, $0.016 \mathrm{mmol}, 4 \mathrm{~mol} \%)$, THF $(0.5 \mathrm{~mL})$ and $n$-propylamine $(0.5 \mathrm{~mL})$. The reaction mixture was heated at $50{ }^{\circ} \mathrm{C}$ for $30 \mathrm{~min}$ and then the volatile solvents were removed in vacuo to give a pale yellow solid. After that, allylic carbonate 1r-1t (0.40 mmol), DBU (60 $\mu \mathrm{L}, 0.4 \mathrm{mmol})$ and THF $(4.0 \mathrm{~mL})$ were added. The reaction mixture was stirred at $50{ }^{\circ} \mathrm{C}$ for $1 \mathrm{~h}$ (the starting material was consumed completely monitored by TLC). The crude reaction mixture was quenched with brine and extracted with DCM $(10 \mathrm{~mL} \times 3)$. The organic layer was concentrated by rotary evaporation. Then to the crude product in DCM $(5 \mathrm{~mL})$ was added $\mathrm{Et}_{3} \mathrm{~N}(140 \mu \mathrm{L}, 1.0 \mathrm{mmol})$ and $(\mathrm{Boc})_{2} \mathrm{O}$ (140 $\mu \mathrm{L}, 0.6 \mathrm{mmol})$. This mixture was stirred for $3 \mathrm{~h}$, quenched with brine and extracted with EA $(10 \mathrm{~mL} \times 3)$. The organic layer was concentrated by rotary evaporation and purified by silica gel column chromatography $(\mathrm{PE} / \mathrm{EA}=20 / 1,10 / 1$, 5/1) to afford the desired products $2 \mathbf{r}-\mathbf{2 t}$. The analytical data of the products are summarized below.

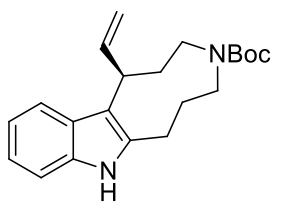

2r, viscous yellow oil, $67 \%$ yield, $98 \%$ ee [Daicel Chiralpak AD-H $(0.46 \mathrm{~cm}$ x 25 $\mathrm{cm}$ ), $n$-hexane/2-propanol $=90 / 10, v=1.0 \mathrm{~mL} \cdot \mathrm{min}^{-1}, \lambda=230 \mathrm{~nm}, \mathrm{t}$ (major) $=6.70$ $\min , \mathrm{t}($ minor $)=7.32 \mathrm{~min}] .[\alpha]_{\mathrm{D}}{ }^{29}=-45.4\left(\mathrm{c}=1.0, \mathrm{CHCl}_{3}\right)$. Two rotamers exist in ${ }^{1} \mathrm{H}$ and ${ }^{13} \mathrm{C}$ NMR. ${ }^{1} \mathrm{H}$ NMR (400 MHz, $\left.\mathrm{CDCl}_{3}\right) \delta 7.95(\mathrm{~d}, 1 \mathrm{H}), 7.55(\mathrm{~d}, J=7.6 \mathrm{~Hz}, 1 \mathrm{H})$, 7.33-7.22 (m, 1H), 7.16-6.98 (m, 2H), 6.37-6.18 (m, 1H), 5.19-5.04 (m, 2H), 
3.79-3.68 (m, 1H), 3.60-3.36 (m, 2H), 3.06-2.90 (m, 1H), 2.83-2.71 (m, 1H), 2.70-1.86 (m, 5H), 1.82-1.65 (m, 1H), $1.44(\mathrm{~d}, 9 \mathrm{H}) .{ }^{13} \mathrm{C}$ NMR (101 MHz, $\left.\mathrm{CDCl}_{3}\right) \delta$ $156.4,156.3,141.6,141.4,136.2,136.1,134.8,134.3,127.0,121.0,120.9,119.9$, 119.5, 118.8, 118.7, 113.5, 113.3, 112.5, 112.0, 110.6, 110.4, 79.2, 52.4, 51.8, 51.1, 36.7, 36.1, 32.1, 31.4, 28.6, 28.4, 27.9, 26.7, 21.9, 21.3. IR (thin film): $v_{\max }\left(\mathrm{cm}^{-1}\right)=$ $3398,3307,2972,2918,2858,1667,1483,1459,1413,1362,1314,1247,1164,1109$, $1015,981,909,859,816,768,739,644$. HRMS-ESI calcd for $\mathrm{C}_{21} \mathrm{H}_{29} \mathrm{~N}_{2} \mathrm{O}_{2}[\mathrm{M}+\mathrm{H}]^{+}$: 341.2224, Found: 341.2225 .

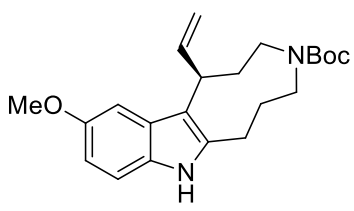

2s, pale yellow solid, m.p. $=175.4-178.4{ }^{\circ} \mathrm{C}, 57 \%$ yield, $96 \%$ ee [Daicel Chiralpak AD-H $(0.46 \mathrm{~cm} \times 25 \mathrm{~cm}), n$-hexane/2-propanol $=90 / 10, v=1.0 \mathrm{~mL} \cdot \mathrm{min}^{-1}$, $\lambda=230 \mathrm{~nm}, \mathrm{t}$ (major) $=6.71 \mathrm{~min}, \mathrm{t}($ minor $)=8.02 \mathrm{~min}] .[\alpha]_{\mathrm{D}}^{28}=-73.4(\mathrm{c}=1.0$, $\mathrm{CHCl}_{3}$ ). Two rotamers exist in ${ }^{1} \mathrm{H}$ and ${ }^{13} \mathrm{C}$ NMR. ${ }^{1} \mathrm{H}$ NMR $\left(400 \mathrm{MHz}, \mathrm{CDCl}_{3}\right) \delta 7.94$ (d, 1H), $7.16(\mathrm{~d}, J=8.8 \mathrm{~Hz}, 1 \mathrm{H}), 7.02(\mathrm{~s}, 1 \mathrm{H}), 6.77(\mathrm{~d}, J=8.4 \mathrm{~Hz}, 1 \mathrm{H}), 6.36-6.15(\mathrm{~m}$, 1H), 5.21-5.02 (m, 2H), $3.82(\mathrm{~d}, 3 \mathrm{H}), 3.75-3.66(\mathrm{~m}, 1 \mathrm{H}), 3.60-3.35(\mathrm{~m}, 2 \mathrm{H}), 3.03-2.86$ (m, 1H), 2.80-2.69 (m, 1H), 2.68-1.62 (m, 6H), $1.44(\mathrm{~d}, 9 \mathrm{H}) .{ }^{13} \mathrm{C}$ NMR (101 MHz, $\left.\mathrm{CDCl}_{3}\right) \delta 174.6,156.5,156.3,153.2,141.5,141.2,135.9,135.4,131.4,127.5,113.5$, 113.3, 112.1, 111.7, 111.04, 110.96, 110.4, 110.0, 102.4, 79.2, 56.0, 55.8, 52.4, 51.7, $51.2,36.5,35.9,31.8,31.1,28.6,28.4,27.9,26.8,22.0,21.4$. IR (thin film): $v_{\max }$ $\left(\mathrm{cm}^{-1}\right)=3299,2971,2921,2857,1667,1589,1484,1416,1363,1322,1215,1166$, 1064, 1039, 988, 898, 877, 853, 825, 791, 770, 697, 651. HRMS-ESI calcd for $\mathrm{C}_{22} \mathrm{H}_{31} \mathrm{~N}_{2} \mathrm{O}_{3}[\mathrm{M}+\mathrm{H}]^{+}:$371.2329, Found: 371.2328.

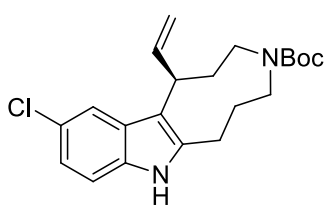

2t, pale yellow solid, m.p. $=185.9-190.4{ }^{\circ} \mathrm{C}, 64 \%$ yield, $98 \%$ ee [Daicel Chiralpak AD-H $(0.46 \mathrm{~cm} \times 25 \mathrm{~cm}), n$-hexane/2-propanol $=90 / 10, v=1.0 \mathrm{~mL} \cdot \mathrm{min}^{-1}$, 
$\lambda=230 \mathrm{~nm}, \mathrm{t}$ (major) $=6.70 \mathrm{~min}, \mathrm{t}($ minor $)=7.79 \mathrm{~min}] .[\alpha]_{\mathrm{D}}{ }^{29}=-74.8(\mathrm{c}=1.0$,

$\mathrm{CHCl}_{3}$ ). Two rotamers exist in ${ }^{1} \mathrm{H}$ and ${ }^{13} \mathrm{C} \mathrm{NMR} .{ }^{1} \mathrm{H}$ NMR $\left(400 \mathrm{MHz}, \mathrm{CDCl}_{3}\right) \delta 8.13$ (d, 1H), $7.49(\mathrm{~d}, J=2.0 \mathrm{~Hz}, 1 \mathrm{H}), 7.22-7.14(\mathrm{~m}, 1 \mathrm{H}), 7.09-7.00(\mathrm{~m}, 1 \mathrm{H}), 6.29-6.12(\mathrm{~m}$, 1H), 5.19-5.04 (m, 2H), 3.77-3.66 (m, 1H), 3.63-3.37 (m, 2H), 3.04-2.87 (m, 1H), 2.83-2.70 (m, 1H), 2.68-2.20 (m, 3H), 2.15-1.83 (m, 2H), 1.82-1.65 (m, 1H), $1.41(\mathrm{~d}$, 9H). ${ }^{13} \mathrm{C}$ NMR $\left(101 \mathrm{MHz}, \mathrm{CDCl}_{3}\right) \delta 156.5,156.3,141.3,141.0,136.7,136.2,134.6$, $134.5,128.3,124.5,124.4,121.4,121.1,119.4,118.9,114.1,113.8,112.5,112.0$, $111.6,111.5,79.5,52.5,51.9,51.9,51.3,36.8,36.0,32.0,31.3,28.7,28.4,27.9,26.7$, 22.4, 21.5. IR (thin film): $v_{\max }\left(\mathrm{cm}^{-1}\right)=3282,2974,2919,2857,1667,1573,1479$, 1415, 1362, 1317, 1294, 1234, 1169, 1121, 1057, 1012, 972, 908, 862, 793, 771, 693, 653, 624. HRMS-ESI calcd for $\mathrm{C}_{21} \mathrm{H}_{28} \mathrm{ClN}_{2} \mathrm{O}_{2}[\mathrm{M}+\mathrm{H}]^{+}:$375.1834, Found: 375.1832 .

\section{VCD and IR experimental}

2a (4.6 mg) was dissolved in $150 \mu \mathrm{LCDCl}_{3}$ and placed in a $\mathrm{BaF}_{2}$ cell with a pathlength of $75 \mu \mathrm{m}$. VCD and IR spectra were then acquired at a resolution of $4 \mathrm{~cm}^{-1}$ for $5 \mathrm{~h}$, using BioTools ChiralIR-2X FT-VCD spectrometer, equipped with a single photoelastic modulation and a mercury cadmium tellurium detector. The racemate was measured under the same conditions to obtain VCD baseline.

\section{VCD and IR calculations}

Initially, molecular model of $R-\mathbf{2 a}$ was built and subjected to a conformational analysis using the Monte Carlo protocol at the molecular mechanic force field MMFF94 level with Compute VOA (BioTools Inc., Jupiter, FL). Within a $20 \mathrm{kcal} / \mathrm{mol}$ energy window, eighteen energetically distinct conformers were predicted. Geometry optimization and frequencies calculation of the conformers were carried out using the B3PW91 hybrid density functional and cc-pVTZ basis set with Gaussian 09 (Gaussian Inc., Wallingford, CT). Boltzmann-population-weighted composite VCD and IR spectra were then generated by Compute VOA. Theoretical curves for $S-\mathbf{2 a}$ were deduced from $R$-2a. As enantiomers, their IR curves are exactly the same and 
VCD spectra are mirror images.

Figure S1 and Figure S2 show the experimental and calculated VCD and IR spectra over the range of $1000-1600 \mathrm{~cm}^{-1}$. A scale factor of 0.979 has been applied to the calculated frequencies to overcome a systematic overestimation of the molecular force constant values by density functional theory (DFT). The assignment of absolute configuration is based on comparisons of the experimental spectra with the theoretical curve obtained by DFT calculations. According to the good agreement between the corresponding spectra, $\mathbf{2 a}$ is assigned to have $\mathrm{R}$ configuration unambiguously.

Quantitative evaluation of this assignment was achieved by Compare VOA (BioTools Inc., Jupiter, FL).The related results, including the spectral similarities and enantiomeric similarity index (the difference between the VCD spectral similarity of the correct and incorrect enantiomers, ESI) are listed in Table S2. Based on the current Compare VOA database, the confidence level of the R assignment for 2a is $99 \%$.

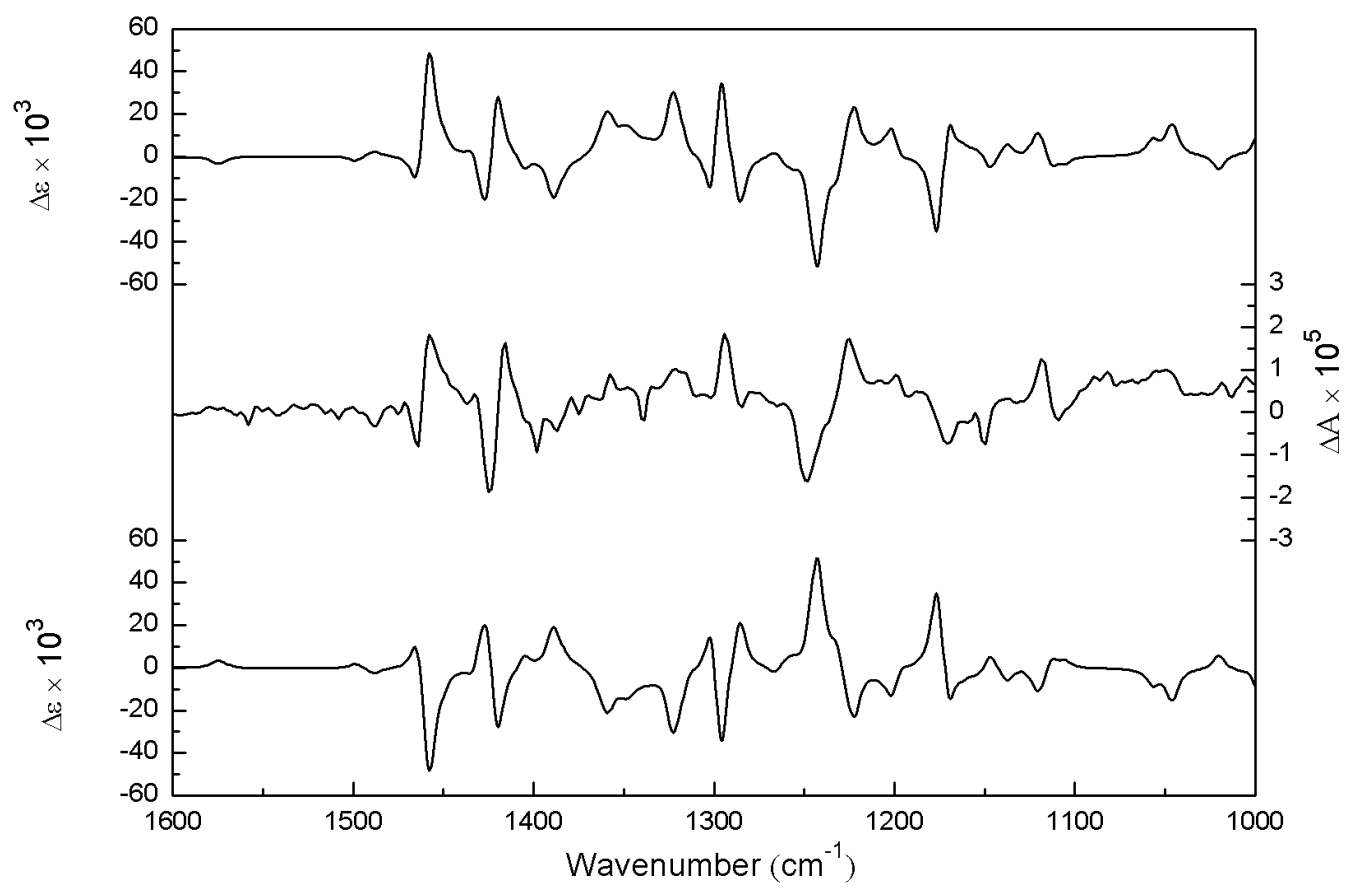

Figure S1 Comparison of the experimental VCD spectrum of $\mathbf{2 a}$ (center) and the calculated VCD spectra of R-2a (top) and S-2a (bottom) 


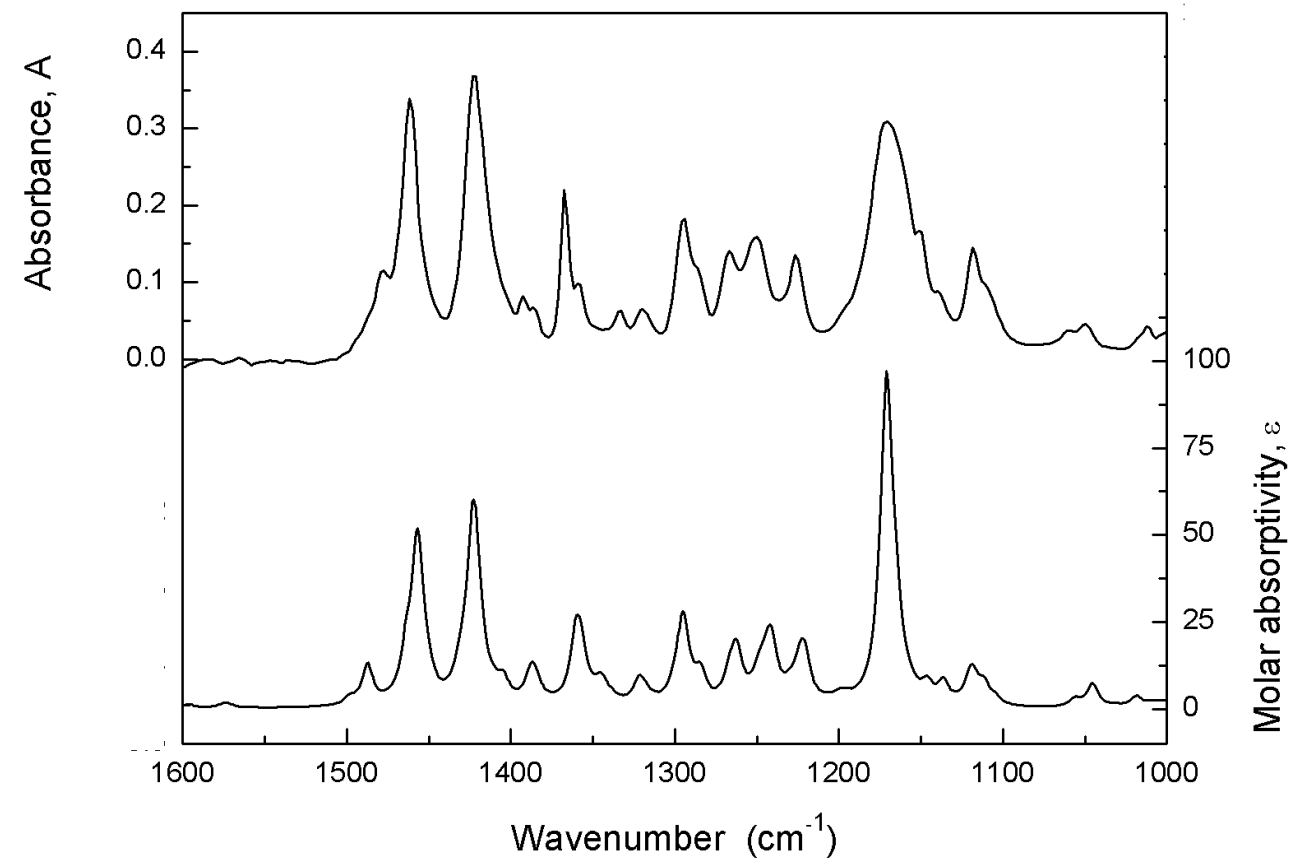

Figure S2 Comparison of the experimental IR spectrum of 2a (top) and the calculated IR spectra for R-2a and S-2a (bottom)

Table S2 Evaluations for the AC assignment of $\mathbf{2 a}$

\begin{tabular}{ccccc}
\hline Calculation Method & ${ }^{\mathrm{a}} S_{I R}$ & ${ }^{\mathrm{b}} S_{E}$ & ${ }^{\mathrm{c}} S_{,-E}$ & ${ }^{\mathrm{d}} E S I$ \\
\hline DFT//B3PW91/cc-pVTZ & 90.2 & 72.5 & 12.2 & 60.3 \\
\hline
\end{tabular}

${ }^{\text {a }}$ Total neighborhood similarity for IR spectra

${ }^{b}$ VCD spectral neighborhood similarity for the correct enantiomer

${ }^{\mathrm{c}} \mathrm{VCD}$ spectral neighborhood similarity for the incorrect enantiomer

${ }^{\mathrm{d}}$ Enantiomeric similarity index

\section{Experiments with the enantiomerically pure substrate 1a:}

(+)-1a and (-)-1a was obtained by Daicel Chiralpak IB $(0.46 \mathrm{~cm} \times 25 \mathrm{~cm})$ $\left[n\right.$-hexane/2-propanol $=80 / 20, v=0.7 \mathrm{~mL} \cdot \min ^{-1}, \lambda=254 \mathrm{~nm}, \mathrm{t}_{1}=9.43 \mathrm{~min}, \mathrm{t}_{2}=$ $11.06 \mathrm{~min}]$.

(+)-1a: $>99 \%$ ee [Daicel Chiralcel OD-H $(0.46 \mathrm{~cm} \quad \mathrm{x} 25 \mathrm{~cm})$, $n$-hexane/2-propanol $\left.=90 / 10, v=1.0 \mathrm{~mL} \cdot \min ^{-1}, \lambda=230 \mathrm{~nm}, \mathrm{t}=15.25 \mathrm{~min}\right] .[\alpha]_{\mathrm{D}}^{28}=$ 
$+22.7\left(\mathrm{c}=1.0, \mathrm{CHCl}_{3}\right)$.

(-)-1a: >99\% ee [Daicel Chiralcel OD-H (0.46 cm x $25 \mathrm{~cm}), n$-hexane/2-propanol $\left.=90 / 10, v=1.0 \mathrm{~mL} \cdot \mathrm{min}^{-1}, \lambda=230 \mathrm{~nm}, \mathrm{t}=24.85 \mathrm{~min}\right]$.

Under the standard conditions, (+)-1a and (-)-1a could almost give the same results in excellent yields and excellent enantioselectivity (Scheme S1-1, 2).

Substrate (+)-1a could only give 1\% ee by employing achiral ligand L9 (Scheme S1-3)

Thus, the chirality of the product was determined by chirality of ligand employed.

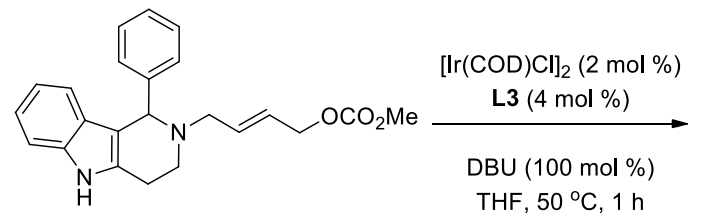

$(+)-1 \mathrm{a}$<smiles>CC(=O)OC/C=C/CN1CCc2[nH]c3ccccc3c2C1c1ccccc1</smiles>

$(-)-1 a$<smiles>COC/C=C/CN1CCc2[nH]c3ccccc3c2C1c1ccccc1</smiles>

$(+)-1 a$

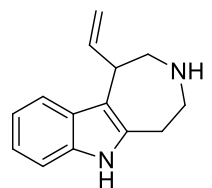

$86 \%$ yield $98 \%$ ee

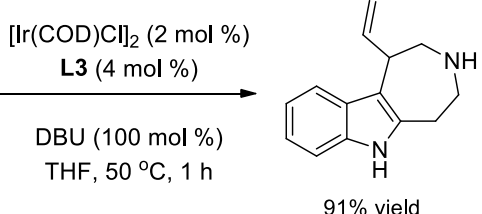

$91 \%$ yield
$99 \%$ ee

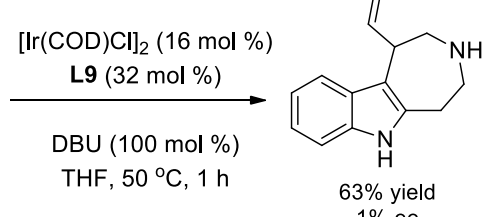

$1 \%$ ee

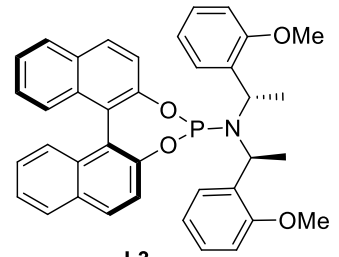

L3
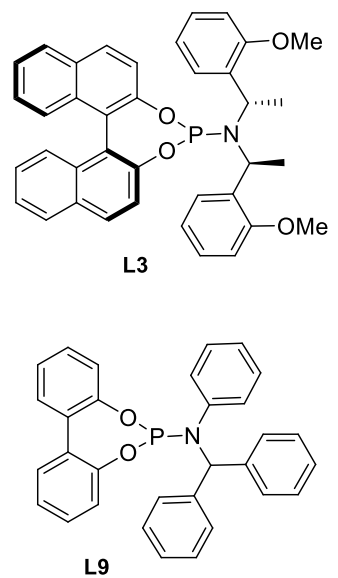

(3)

Scheme S1. Experiments with the enantiomerically pure substrate 1a

\section{Gram-Scale Synthesis of 2a}

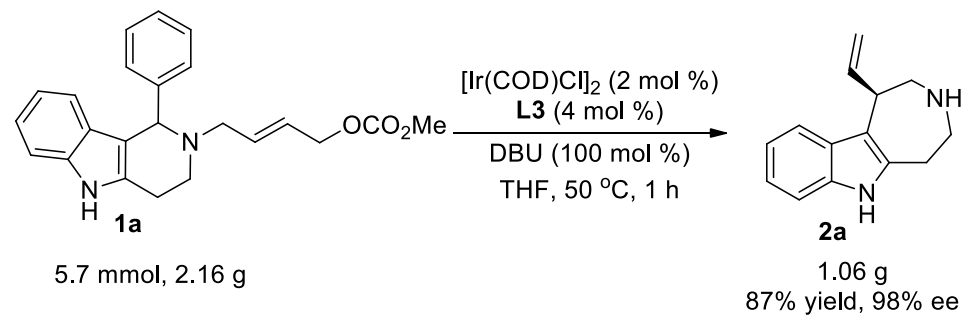


A flame dried Schlenk tube was cooled to rt and filled with argon. To this flask were added $[\operatorname{Ir}(\mathrm{COD}) \mathrm{Cl}]_{2}(77.6 \mathrm{mg}, 0.115 \mathrm{mmol}, 2 \mathrm{~mol} \%)$, phosphoramidite ligand $\mathbf{L 3}$ (137.3 mg, $0.229 \mathrm{mmol}, 4 \mathrm{~mol} \%)$, THF (5 mL) and $n$-propylamine $(5 \mathrm{~mL})$. The reaction mixture was heated at $50{ }^{\circ} \mathrm{C}$ for $30 \mathrm{~min}$ and then the volatile solvents were removed in vacuo to give a pale yellow solid. After that, allylic carbonate $1 \mathbf{a}(2.16 \mathrm{~g}$, $5.73 \mathrm{mmol})$, DBU $(0.86 \mathrm{~mL}, 5.73 \mathrm{mmol})$ and THF $(57 \mathrm{~mL})$ were added. The reaction mixture was stirred at $50{ }^{\circ} \mathrm{C}$ for $1 \mathrm{~h}$ (the starting material was consumed completely monitored by TLC). The crude reaction mixture was quenched with brine and extracted with DCM $(30 \mathrm{~mL} \times 3)$. The organic layer was concentrated by rotary evaporation. Then the residue was purified by $\mathrm{Al}_{2} \mathrm{O}_{3}$ column chromatography $(\mathrm{DCM} / \mathrm{MeOH}=50 / 1,20 / 1)$ to afford the desired product $\mathbf{2 a}(1.06 \mathrm{~g}, 87 \%$ yield, $98 \%$ ee).

\section{Transformation of the Product:}

1) Procedure for Pd/C-Catalyzed Hydrogenation of Compound 2a

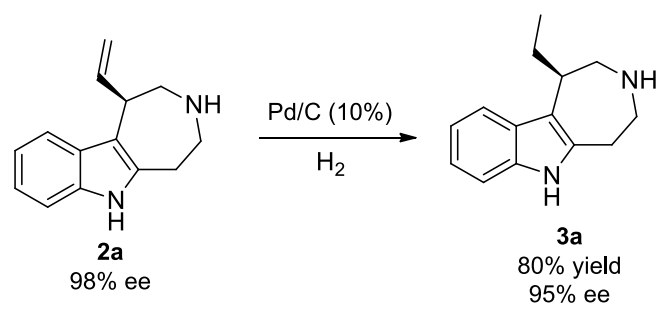

$\mathrm{Pd} / \mathrm{C}$ (38.9 mg, palladium on activated carbon, 10\% Pd basis, 0.1 equiv) was added to a solution of $\mathbf{2 a}(79.1 \mathrm{mg}, 0.37 \mathrm{mmol}, 1$ equiv, $98 \%$ ee) in anhydrous $\mathrm{MeOH}$ $(5.0 \mathrm{~mL})$. The reaction mixture was stirred under $\mathrm{H}_{2}$ atmosphere $(1 \mathrm{~atm})$ at $\mathrm{rt}$ for $6 \mathrm{~h}$. After the reaction was complete (monitored by TLC), the crude reaction mixture was filtrated with celite and washed with $\mathrm{MeOH}$. The solvent was removed under reduced pressure. Then the residue was purified by $\mathrm{Al}_{2} \mathrm{O}_{3}$ column chromatography $(\mathrm{DCM} / \mathrm{MeOH}=50 / 1,20 / 1)$ to afford the desired product 3a. Viscous yellow oil, 63.6 $\mathrm{mg}, 80 \%$ yield, $95 \%$ ee [Daicel Chiralcel OD-H $(0.46 \mathrm{~cm}$ x $25 \mathrm{~cm})$, $n$-hexane/2-propanol/diethylamine $=96 / 4 / 0.1, v=0.5 \mathrm{~mL} \cdot \mathrm{min}^{-1}, \lambda=230 \mathrm{~nm}, \mathrm{t}$ (minor) 
$=32.53 \mathrm{~min}, \mathrm{t}$ (major) $=34.80 \mathrm{~min}] .[\alpha]_{\mathrm{D}}{ }^{26}=+79.6\left(\mathrm{c}=0.25, \mathrm{CHCl}_{3}\right) .{ }^{1} \mathrm{H} \mathrm{NMR}(400$ $\left.\mathrm{MHz}, \mathrm{CDCl}_{3}\right) \delta 7.97(\mathrm{~s}, 1 \mathrm{H})$, 7.49-7.43 (m, 1H), 7.25-7.22 (m, 1H), 7.11-7.03 (m, 2H), $3.40(\mathrm{dd}, J=13.2,3.2 \mathrm{~Hz}, 1 \mathrm{H}), 3.32(\mathrm{dt}, J=12.8,3.6 \mathrm{~Hz}, 1 \mathrm{H}), 3.15-3.04(\mathrm{~m}, 1 \mathrm{H})$, 2.98-2.87 (m, 2H), $2.80(\mathrm{t}, J=12.8 \mathrm{~Hz}, 1 \mathrm{H}), 2.72-2.65(\mathrm{~m}, 1 \mathrm{H}), 1.71(\mathrm{dt}, J=14.8$, $7.6 \mathrm{~Hz}, 2 \mathrm{H}), 0.93(\mathrm{t}, J=7.6 \mathrm{~Hz}, 3 \mathrm{H}) .{ }^{13} \mathrm{C} \mathrm{NMR}\left(101 \mathrm{MHz}, \mathrm{CDCl}_{3}\right) \delta 135.2,134.5$, $129.6,120.8,119.1,118.0,117.2,110.4,53.1,48.8,38.8,32.7,26.2,13.0$. IR (thin

film): $v_{\max }\left(\mathrm{cm}^{-1}\right)=3398,3189,3053,2956,2924,2868,1644,1618,1490,1458$, 1336, 1263, 1227, 1184, 1143, 1087, 1010, 822, 799, 739. HRMS-ESI calcd for $\mathrm{C}_{14} \mathrm{H}_{19} \mathrm{~N}_{2}[\mathrm{M}+\mathrm{H}]^{+}:$215.1543, Found: 215.1543.

\section{2) Procedure for Synthesis of 5 a}
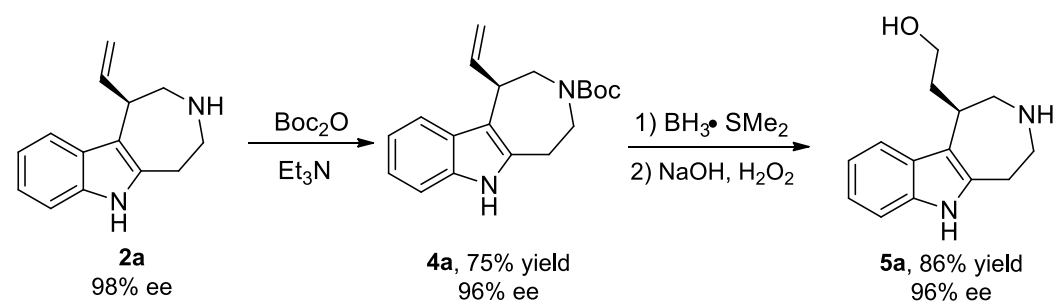

$(\mathrm{Boc})_{2} \mathrm{O}(0.17 \mathrm{~mL}, 0.73 \mathrm{mmol}, 1.2$ equiv) was added to a solution of $\mathbf{2 a}(128.4$ $\mathrm{mg}, 0.6 \mathrm{mmol}, 1$ equiv, $98 \%$ ee $)$ and $\mathrm{Et}_{3} \mathrm{~N}(0.17 \mathrm{~mL}, 1.21 \mathrm{mmol}, 2$ equiv) in DCM (5 $\mathrm{mL}$ ). This mixture was stirred for $6 \mathrm{~h}$ until complete conversion (monitored by TLC). Then the crude reaction mixture was quenched with brine and extracted with EtOAc $(10 \mathrm{~mL} \times 3)$. The organic layer was dried over $\mathrm{Na}_{2} \mathrm{SO}_{4}$, filtered and concentrated by rotary evaporation. The residue was purified by silica gel column chromatography $(\mathrm{PE} / \mathrm{EA}=20 / 1,10 / 1,5 / 1)$ to afford the desired product 4a.Viscous yellow oil, 141.6 mg, $75 \%$ yield, $96 \%$ ee [Daicel Chiralcel OD-H $(0.46 \mathrm{~cm} \quad \mathrm{x} 25 \mathrm{~cm})$, $n$-hexane/2-propanol $=90 / 10, v=1.0 \mathrm{~mL} \cdot \min ^{-1}, \lambda=230 \mathrm{~nm}, \mathrm{t}($ minor $)=7.04 \mathrm{~min}, \mathrm{t}$ $($ major $)=9.06 \mathrm{~min}] .[\alpha]_{\mathrm{D}}{ }^{28}=+1.5\left(\mathrm{c}=0.25, \mathrm{CHCl}_{3}\right) .{ }^{1} \mathrm{H} \mathrm{NMR}\left(400 \mathrm{MHz}, d_{6}-\mathrm{DMSO}\right.$, $\left.80{ }^{\circ} \mathrm{C}\right) \delta 10.54(\mathrm{~s}, 1 \mathrm{H}), 7.33(\mathrm{~d}, J=8.0 \mathrm{~Hz}, 1 \mathrm{H}), 7.24(\mathrm{~d}, J=7.6 \mathrm{~Hz}, 1 \mathrm{H}), 7.06-6.85$ (m, 2H), $5.95(\mathrm{ddd}, J=16.8,10.0,6.8 \mathrm{~Hz}, 1 \mathrm{H}), 4.90(\mathrm{~d}, J=10.0 \mathrm{~Hz}, 1 \mathrm{H}), 4.78(\mathrm{~d}, J$ $=16.8 \mathrm{~Hz}, 1 \mathrm{H}), 4.16(\mathrm{dd}, J=13.4,4.4 \mathrm{~Hz}, 1 \mathrm{H}), 3.97(\mathrm{~d}, J=12.4 \mathrm{~Hz}, 1 \mathrm{H}), 3.84(\mathrm{~s}$, 
1H), $3.35(\mathrm{dd}, J=14.0,2.8 \mathrm{~Hz}, 1 \mathrm{H}), 3.26-3.12(\mathrm{~m}, 1 \mathrm{H}), 3.08-2.97$ (m, 2H), 2.94-2.83 (m, 1H), 1.42 (s, 9H). ${ }^{13} \mathrm{C} \mathrm{NMR} \mathrm{(101} \mathrm{MHz,} d_{6}$-DMSO, $\left.80{ }^{\circ} \mathrm{C}\right) \delta 154.1,139.4,135.0$, 134.5, 128.2, 119.7, 117.8, 117.1, 113.5, 110.6, 110.1, 78.2, 50.4, 46.0, 40.9, 27.7, 27.5. IR (thin film): $v_{\max }\left(\mathrm{cm}^{-1}\right)=3402,3308,2974,2924,1663,1460,1419,1363$, 1319, 1294, 1249, 1226, 1162, 1114, 1051, 992, 940, 912, 857, 766, 740, 630. HRMS-ESI calcd for $\mathrm{C}_{19} \mathrm{H}_{25} \mathrm{~N}_{2} \mathrm{O}_{2}[\mathrm{M}+\mathrm{H}]^{+}:$313.1911, Found: 313.1911 .

$\mathrm{BH}_{3} \cdot \mathrm{SMe}_{2}(0.28 \mathrm{~mL}, 2.0 \mathrm{M}, 0.55 \mathrm{mmol}, 3$ equiv) was added to a solution of $\mathbf{4 a}$ (57.7 mg, $0.18 \mathrm{mmol}, 1$ equiv, $96 \%$ ee) in dry THF $(2 \mathrm{~mL})$ at $0{ }^{\circ} \mathrm{C}$. The mixture was stirred for $2 \mathrm{~h}$ at $\mathrm{rt}$ and an aqueous solution of $1 \mathrm{M} \mathrm{NaOH}(0.81 \mathrm{~mL}, 0.81 \mathrm{mmol}, 4.5$ equiv) and $30 \% \mathrm{H}_{2} \mathrm{O}_{2}(0.12 \mathrm{~mL}, 6$ equiv) were added. After stirring for $3 \mathrm{~h}$, the mixture was extracted twice with EtOAc $(10 \mathrm{~mL} \times 3)$. The organic layer was dried over $\mathrm{Na}_{2} \mathrm{SO}_{4}$, filtered and concentrated by rotary evaporation. The residue was purified by silica gel column chromatography $(\mathrm{PE} / \mathrm{EA}=1 / 1)$ to afford the desired product 5a. Pale yellow solid, $52.5 \mathrm{mg}$, m.p. $=83.1-86.8{ }^{\circ} \mathrm{C}, 86 \%$ yield, $96 \%$ ee [Daicel Chiralpak AD-H (0.46 cm x $25 \mathrm{~cm})$, n-hexane/2-propanol = 80/20, $v=1.0$ $\mathrm{mL} \cdot \min -1, \lambda=230 \mathrm{~nm}, \mathrm{t}$ (major) $=7.07 \mathrm{~min}, \mathrm{t}($ minor $)=10.00 \mathrm{~min}] .[\alpha]_{\mathrm{D}}{ }^{27}=+50.3$ (c $\left.=0.5, \mathrm{CHCl}_{3}\right) .{ }^{1} \mathrm{H} \mathrm{NMR}\left(600 \mathrm{MHz}, d_{6}\right.$-DMSO, $\left.80{ }^{\circ} \mathrm{C}\right) \delta 10.47(\mathrm{~s}, 1 \mathrm{H}), 7.45(\mathrm{~d}, J=$ $8.4 \mathrm{~Hz}, 1 \mathrm{H}), 7.25-7.22(\mathrm{~m}, 1 \mathrm{H}), 6.99(\mathrm{td}, J=7.2,1.2 \mathrm{~Hz}, 1 \mathrm{H}), 6.93(\mathrm{td}, J=7.2,1.2$ $\mathrm{Hz}, 1 \mathrm{H}), 4.29-4.03(\mathrm{~m}, 3 \mathrm{H}), 3.50-3.43(\mathrm{~m}, 2 \mathrm{H}), 3.31-3.25(\mathrm{~m}, 1 \mathrm{H}), 3.24-3.14(\mathrm{~m}, 1 \mathrm{H})$, 3.06-2.97 (m, 2H), 2.90-2.83 (m, 1H), 1.86-1.76 (m, 1H), 1.66-1.57 (m, 1H), $1.46(\mathrm{~s}$, $9 \mathrm{H}) .{ }^{13} \mathrm{C}$ NMR (151 MHz, $d_{6}$-DMSO, $\left.80{ }^{\circ} \mathrm{C}\right) \delta 154.4,134.3,134.2,128.4,119.6$, $117.8,117.1,114.1,110.0,78.3,59.0,49.9,46.4,36.2,32.7,28.0,27.8$. IR (thin film): $v_{\max }\left(\mathrm{cm}^{-1}\right)=3399,3308,2963,2925,2854,1661,1461,1421,1365,1258,1228$, 1161, 1116, 1028, 938, 858, 799, 742, 700, 666. HRMS-ESI calcd for $\mathrm{C}_{19} \mathrm{H}_{27} \mathrm{~N}_{2} \mathrm{O}_{3}$ $[\mathrm{M}+\mathrm{H}]^{+}:$331.2016, Found: 331.2016.

\section{Mechanistic Study:}

\section{Isolation of Intermediate}




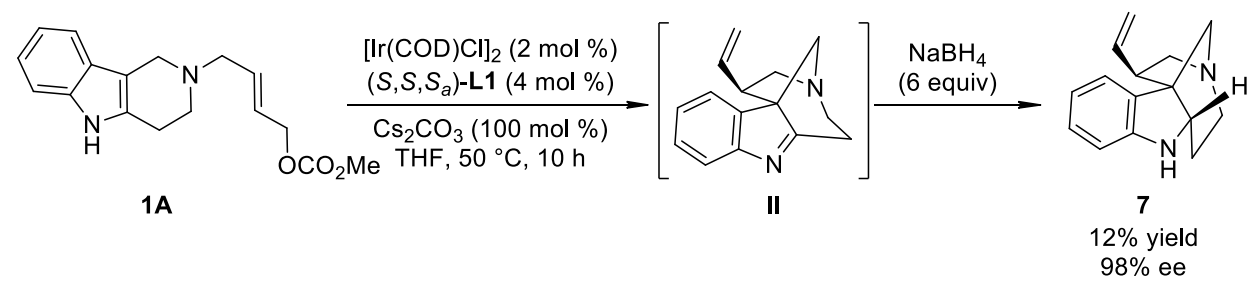

A flame dried Schlenk tube was cooled to rt and filled with argon. To this flask were added $[\mathrm{Ir}(\mathrm{COD}) \mathrm{Cl}]_{2}(2.7 \mathrm{mg}, 0.004 \mathrm{mmol}, 2 \mathrm{~mol} \%)$, phosphoramidite ligand $\mathbf{L 1}$ (4.3 mg, $0.008 \mathrm{mmol}, 4 \mathrm{~mol} \%)$, THF $(0.5 \mathrm{~mL})$ and $n$-propylamine $(0.5 \mathrm{~mL})$. The reaction mixture was heated at $50{ }^{\circ} \mathrm{C}$ for $30 \mathrm{~min}$ and then the volatile solvents were removed in vacuo to give a pale yellow solid. After that, allylic carbonate 1A (60.1 $\mathrm{mg}, 0.20 \mathrm{mmol}), \mathrm{Cs}_{2} \mathrm{CO}_{3}(65.2 \mathrm{mg}, 0.20 \mathrm{mmol})$ and THF $(2.0 \mathrm{~mL})$ were added. The reaction mixture was stirred at $50{ }^{\circ} \mathrm{C}$ for $10 \mathrm{~h}$ (the starting material was consumed monitored by TLC). To this flask were directly added $\mathrm{NaBH}_{4}$ (45 mg, $1.2 \mathrm{mmol}, 6$ equiv) and $\mathrm{MeOH}(0.1 \mathrm{~mL})$ at $0{ }^{\circ} \mathrm{C}$. After stirring for $1 \mathrm{~h}$ at $\mathrm{rt}$, the crude reaction mixture was quenched with water and extracted with $\mathrm{CH}_{2} \mathrm{Cl}_{2}(10 \mathrm{~mL} \times 3)$. The organic layer was concentrated by rotary evaporation and purified by silica gel column chromatography $(\mathrm{PE} / \mathrm{EA}=2 / 1,1 / 1)$ to afford the desired product 7 . Viscous yellow oil, $11.2 \mathrm{mg}, 12 \%$ yield, $98 \%$ ee [Daicel Chiralpak IC $(0.46 \mathrm{~cm}$ x $25 \mathrm{~cm})$, $n$-hexane/2-propanol $=90 / 10, v=1.0 \mathrm{~mL} \cdot \min ^{-1}, \lambda=254 \mathrm{~nm}, \mathrm{t}$ (major) $=22.20 \mathrm{~min}, \mathrm{t}$ $($ minor $)=29.35 \mathrm{~min}] .[\alpha]_{\mathrm{D}}{ }^{29}=-4.3\left(\mathrm{c}=0.25, \mathrm{CHCl}_{3}\right) .{ }^{1} \mathrm{H} \mathrm{NMR}\left(400 \mathrm{MHz}, \mathrm{CDCl}_{3}\right) \delta$ $7.09(\mathrm{td}, J=7.6,1.2 \mathrm{~Hz}, 1 \mathrm{H}), 6.98-6.94(\mathrm{~m}, 1 \mathrm{H}), 6.80-6.72(\mathrm{~m}, 2 \mathrm{H}), 5.51$ (ddd, $J=$ 16.8, 10.4, 7.6 Hz, 1H), 4.87 (d, $J=10.4 \mathrm{~Hz}, 1 \mathrm{H}), 4.73$ (d, $J=16.8 \mathrm{~Hz}, 1 \mathrm{H}), 3.99$ (s, 1H), $3.64(\mathrm{dd}, J=11.6,1.2 \mathrm{~Hz}, 1 \mathrm{H}), 3.57(\mathrm{dd}, J=11.6,5.2 \mathrm{~Hz}, 1 \mathrm{H}), 3.28-3.15(\mathrm{~m}$, 4H), $3.14-3.03(\mathrm{~m}, 1 \mathrm{H}), 2.97(\mathrm{~d}, J=11.6 \mathrm{~Hz}, 1 \mathrm{H}), 2.23-2.06(\mathrm{~m}, 2 \mathrm{H}) .{ }^{13} \mathrm{C}$ NMR $(101$ $\left.\mathrm{MHz}, \mathrm{CDCl}_{3}\right) \delta 150.9,137.8,128.6,127.9,124.9,119.4,116.9,110.9,67.0,66.5$, 63.0, 59.2, 56.5, 42.9, 25.3. IR (thin film): $v_{\max }\left(\mathrm{cm}^{-1}\right)=3314,2993,2962,2924,2853$, $1639,1609,1462,1384,1314,1288,1226,1165,1081,1033,988,925,895,863,784$, 767, 747, 676, 654, 618. HRMS-ESI calcd for $\mathrm{C}_{15} \mathrm{H}_{19} \mathrm{~N}_{2}[\mathrm{M}+\mathrm{H}]^{+}:$227.1543, Found: 227.1547 . 


\section{Reference:}

(1) Alexandre, B.; Laurence, G.; Raymond, H.; Jean-Pierre, H. J. Heterocycl. Chem. 2006, 43,571 .

(2) Spadoni, G.; Balsamini, C.; Bedini, A.; Diamantini, G.; Di Giacomo, B.; Tontini, A.; Tarzia, G.; Mor, M.; Plazzi, P. V.; Rivara, S.; Nonno, R.; Pannacci, M.; Lucini, V.; Fraschini, F.; Stankov, B. M. J. Med. Chem. 1998, 41, 3624.

(3) Outlaw, V. K.; Townsend, C. A. Org. Lett. 2014, 16, 6334.

\section{NMR Spectra of $\mathbf{1 A}$}

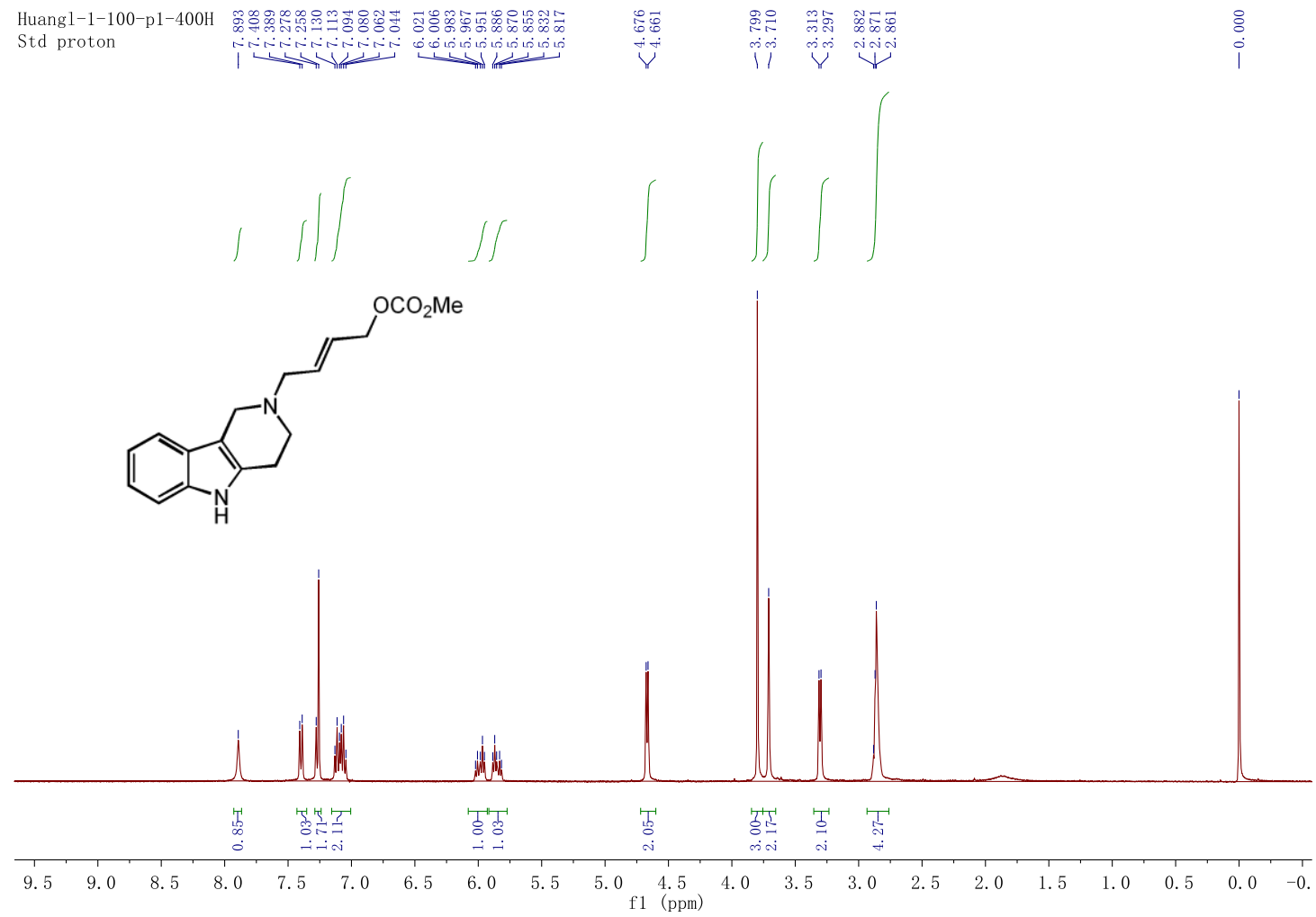



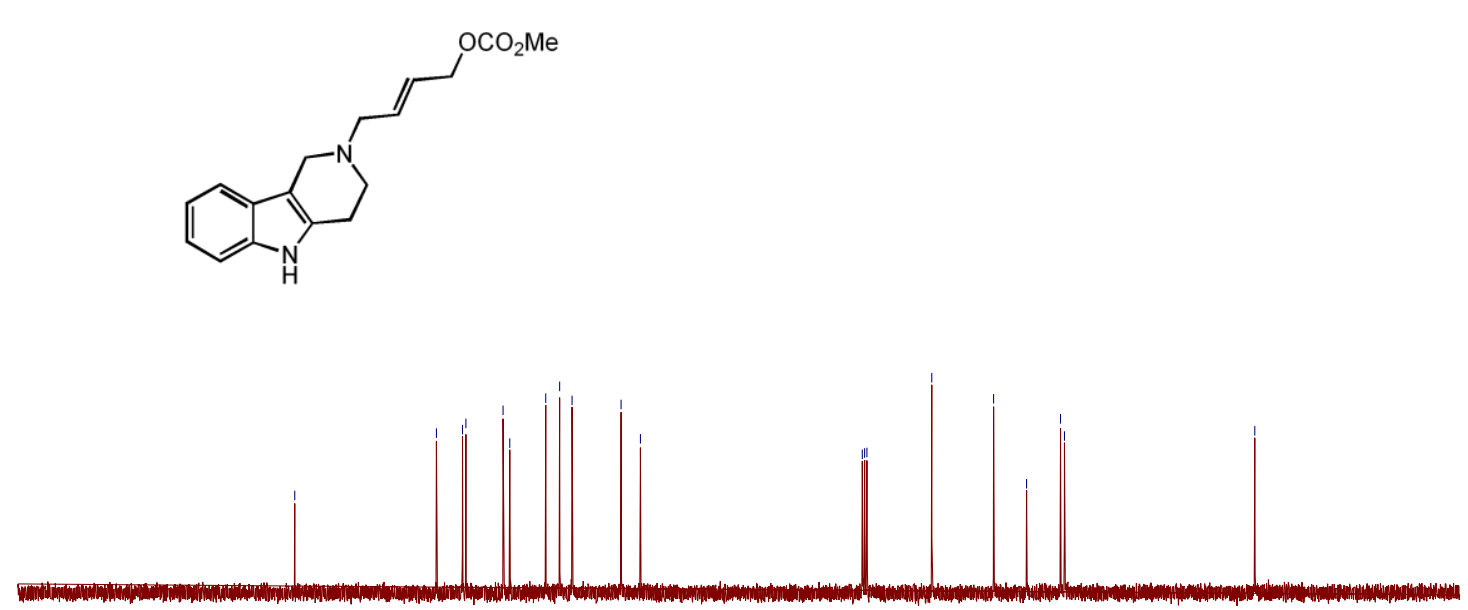

$\begin{array}{rrrrrrrrrrr}190 & 180 & 170 & 160 & 150 & 140 & 130 & 120 & 110 & 100 & 90 \\ & 11 & & & & & & & \end{array}$

\section{NMR Spectra of $\mathbf{1 a}$}

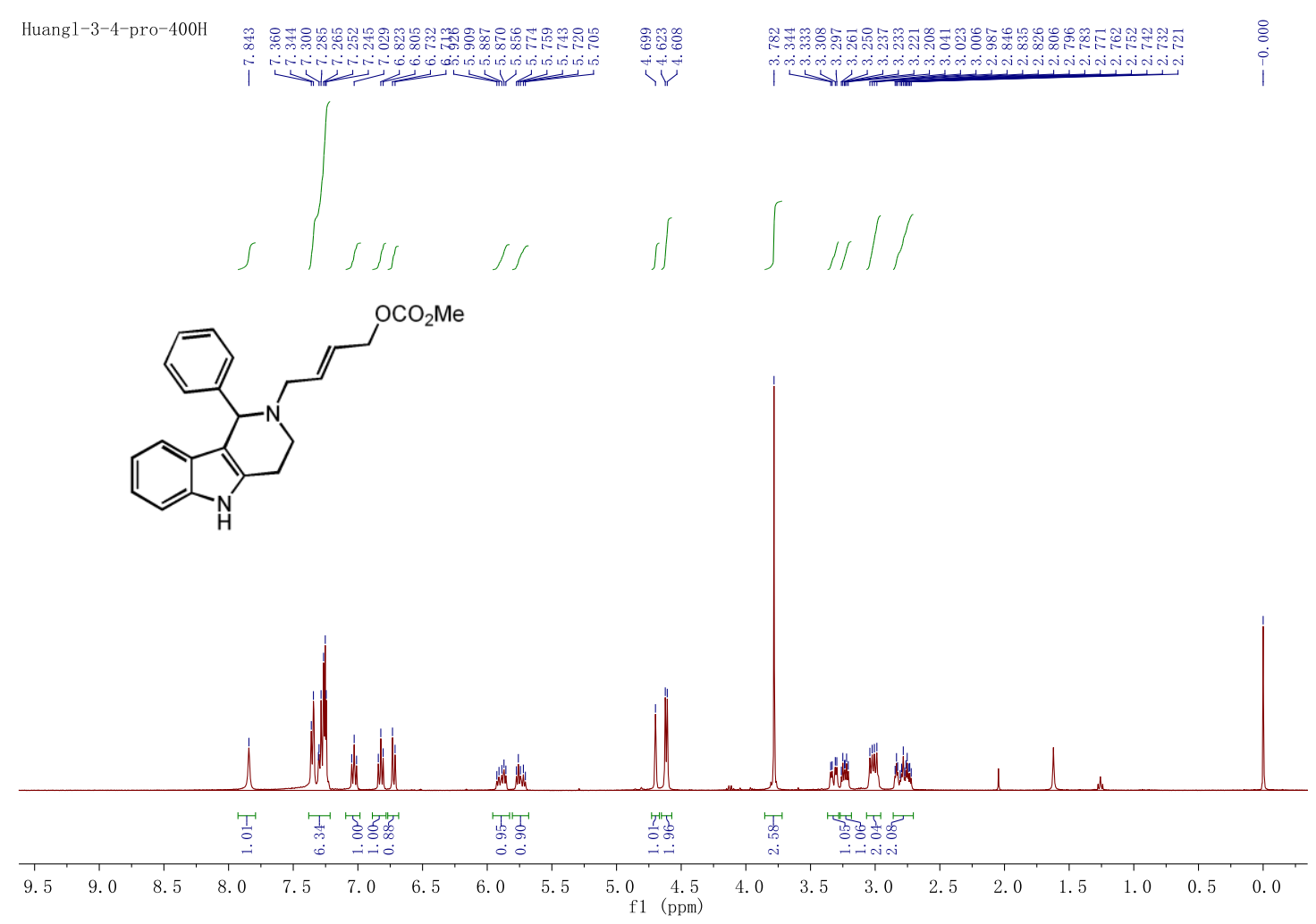



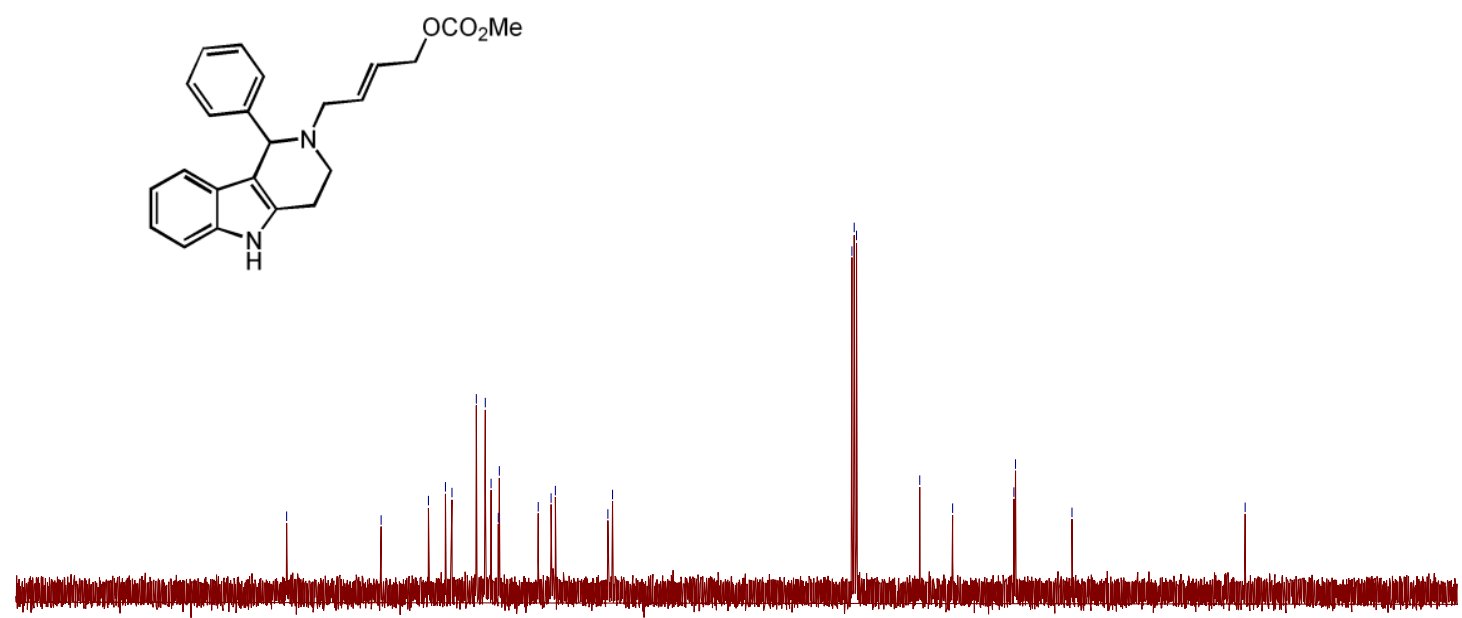

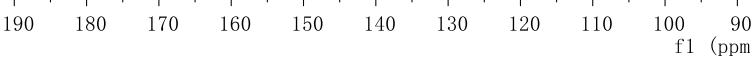

HPLC Chromatographs of (rac)-1a

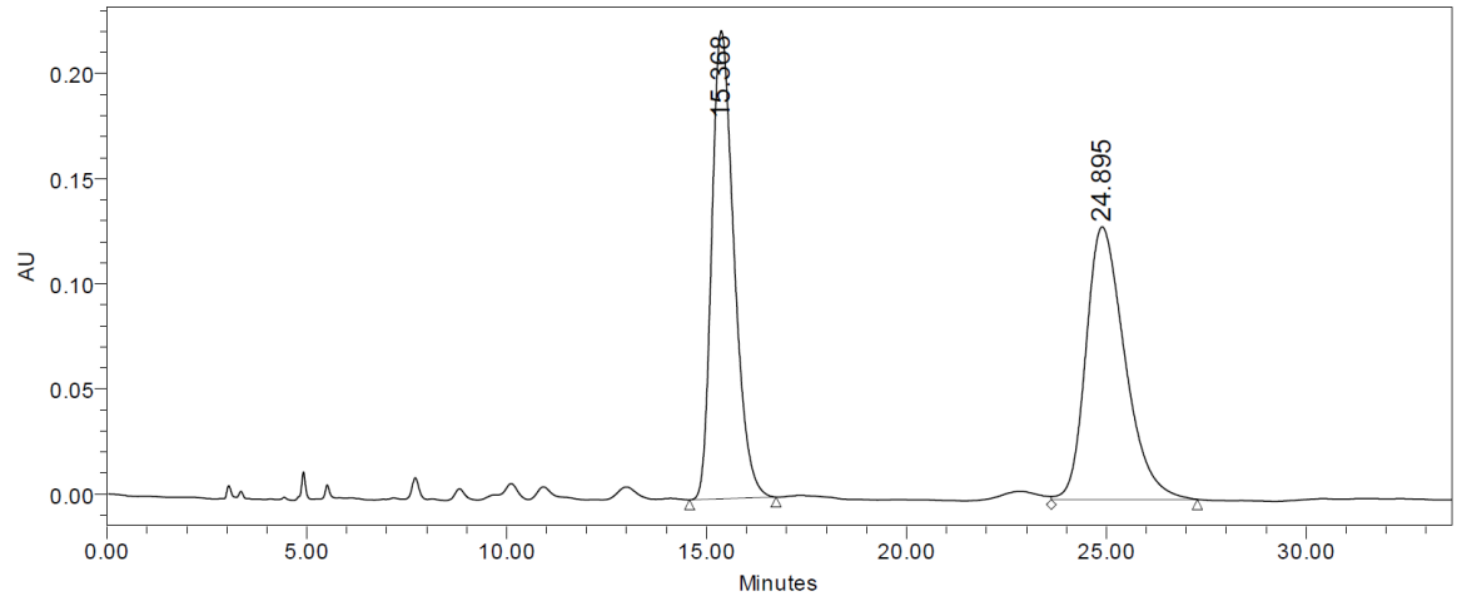

Channel: W2489 ChA; Processed Channel: W2489 ChA 230nm; Injection: 1; Date Acquired: 1/11/2016 12:15:54 PM CST; Result Id: 2873; Processing Method: drthseg

Peak Name:

\begin{tabular}{|c|r|c|c|r|c|}
\hline & Injection & RT & Area & $\%$ Area & Height \\
\hline 1 & 1 & 24.895 & 8776544 & 50.46 & 129773 \\
\hline 2 & 1 & 15.368 & 8617951 & 49.54 & 222797 \\
\hline Mean & & 20.131 & & & \\
\hline Std. Dev. & & 6.737 & & & \\
\hline \% RSD & & 33.46 & & & \\
\hline
\end{tabular}


HPLC Chromatographs of (+)-1a

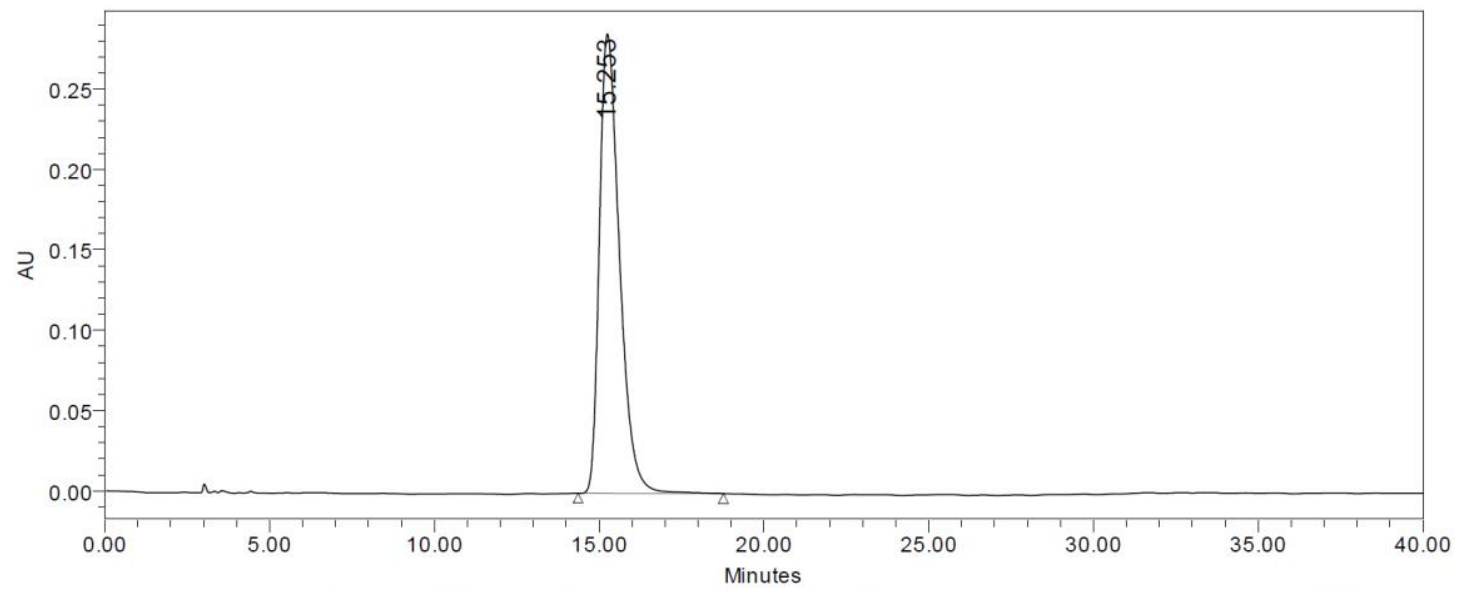

Channel: W2489 ChA; Processed Channel: W2489 ChA 230nm; Injection: 1; Date Acquired: 1/13/2016 11:49:42 AM CST; Result Id: 3081; Processing Method: FGHSFHG

Peak Name:

\begin{tabular}{|c|r|c|c|c|c|}
\hline & Injection & RT & Area & $\%$ Area & Height \\
\hline 1 & 1 & 15.253 & 12123117 & 100.00 & 285801 \\
\hline Mean & & 15.253 & & & \\
\hline Std. Dev. & & & & & \\
\hline$\%$ RSD & & & & & \\
\hline
\end{tabular}

HPLC Chromatographs of (-)-1a

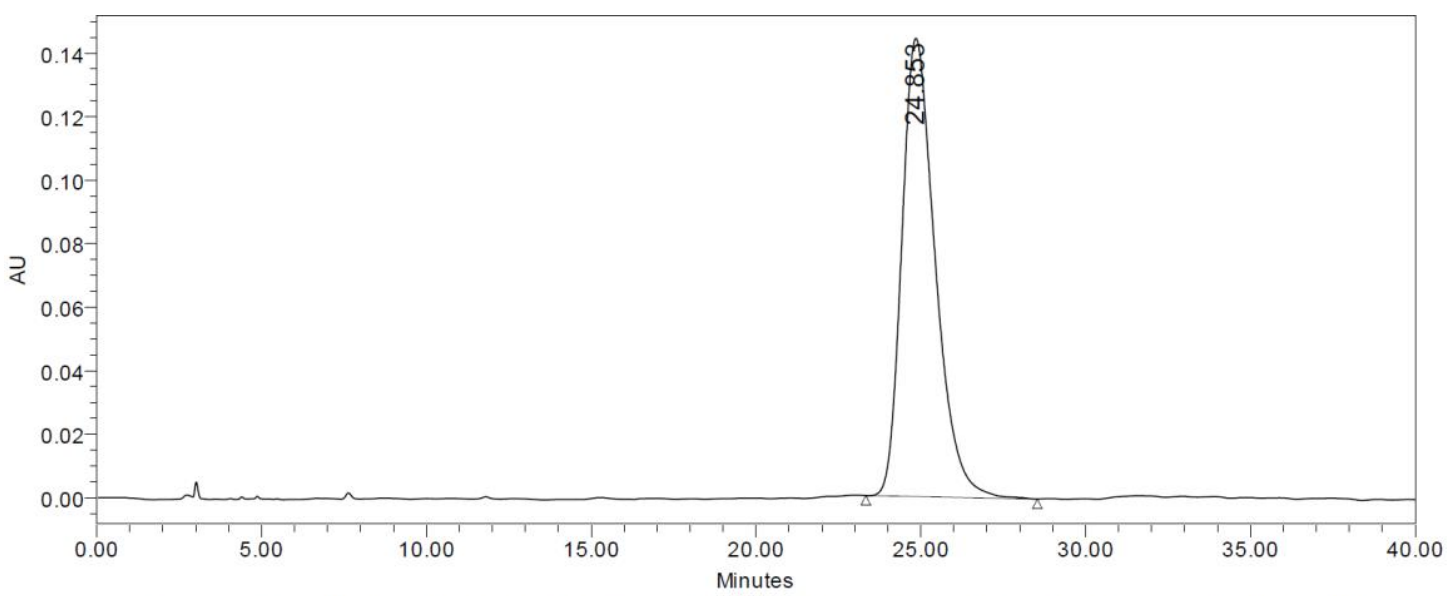

Channel: W2489 ChA; Processed Channel: W2489 ChA 230nm; Injection: 1; Date Acquired: 1/15/2016 5:29:06 PM CST; Result Id: 3083; Processing Method: FGJSZFHDSX

Peak Name:

\begin{tabular}{|c|r|c|c|c|c|}
\hline & Injection & RT & Area & $\%$ Area & Height \\
\hline 1 & 1 & 24.853 & 10157737 & 100.00 & 144272 \\
\hline Mean & & 24.853 & & & \\
\hline Std. Dev. & & & & & \\
\hline$\%$ RSD & & & & & \\
\hline
\end{tabular}

\section{NMR Spectra of 1a'}



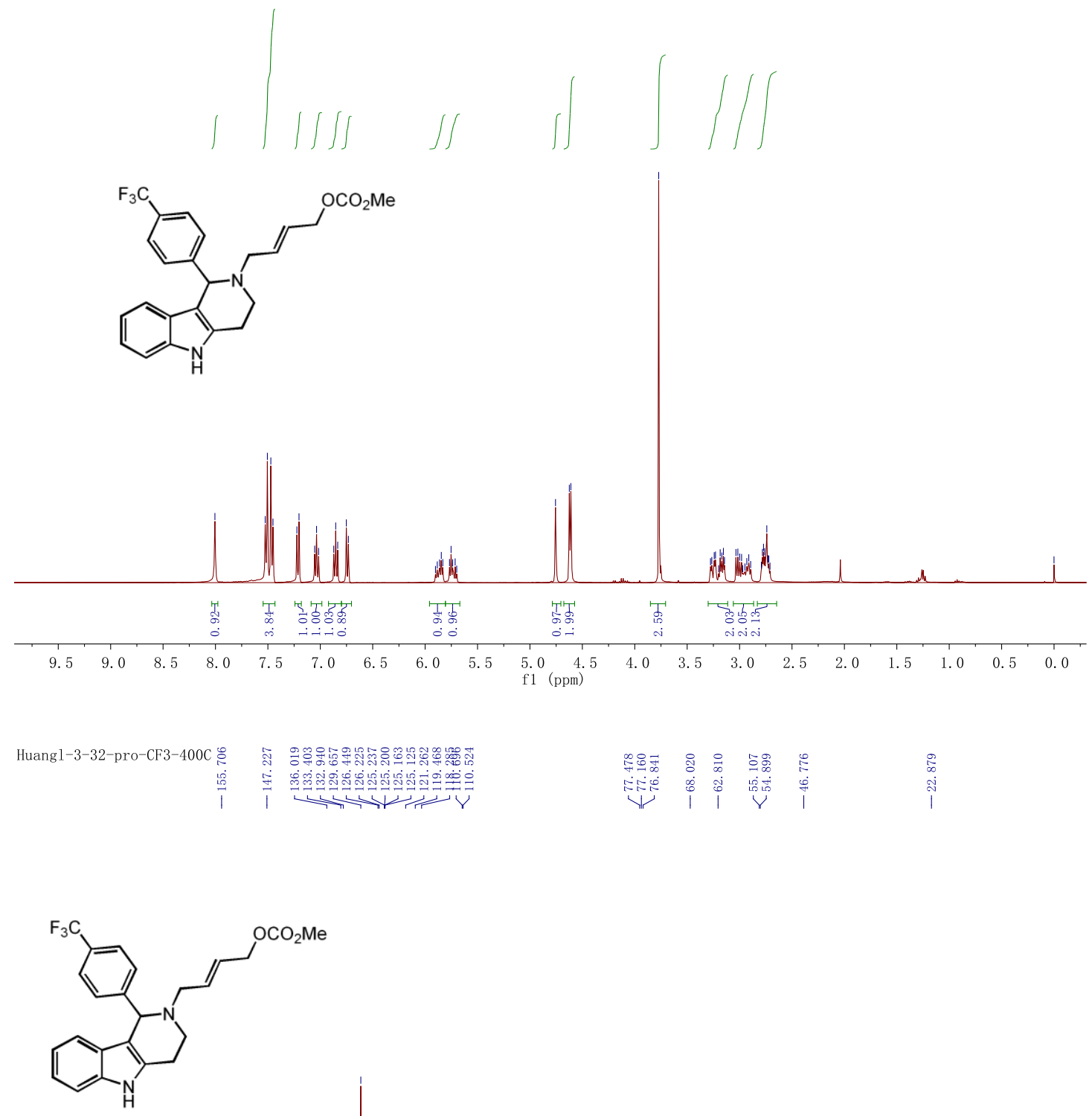
Huang 1-3-32-pro-400F Std Fluorine

ga

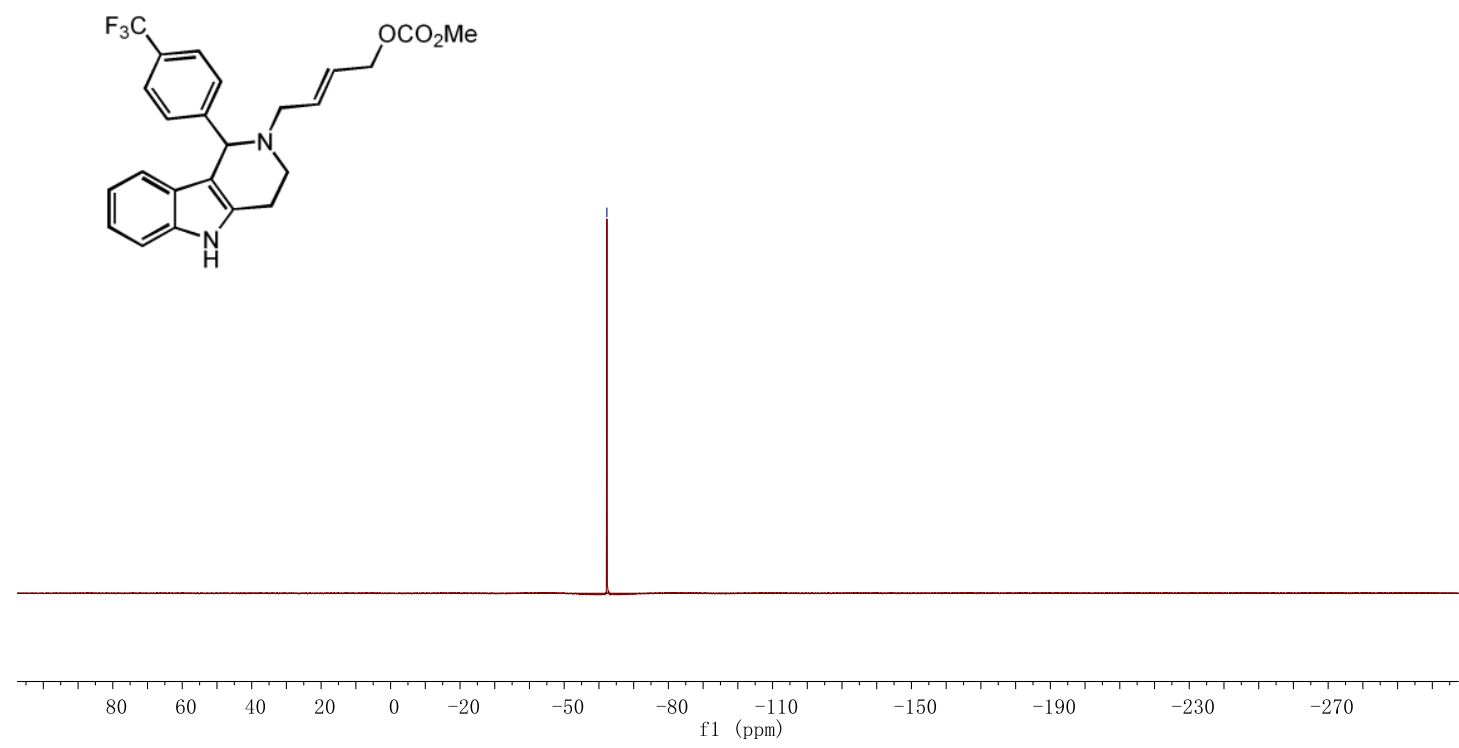

\section{NMR Spectra of 1a"}

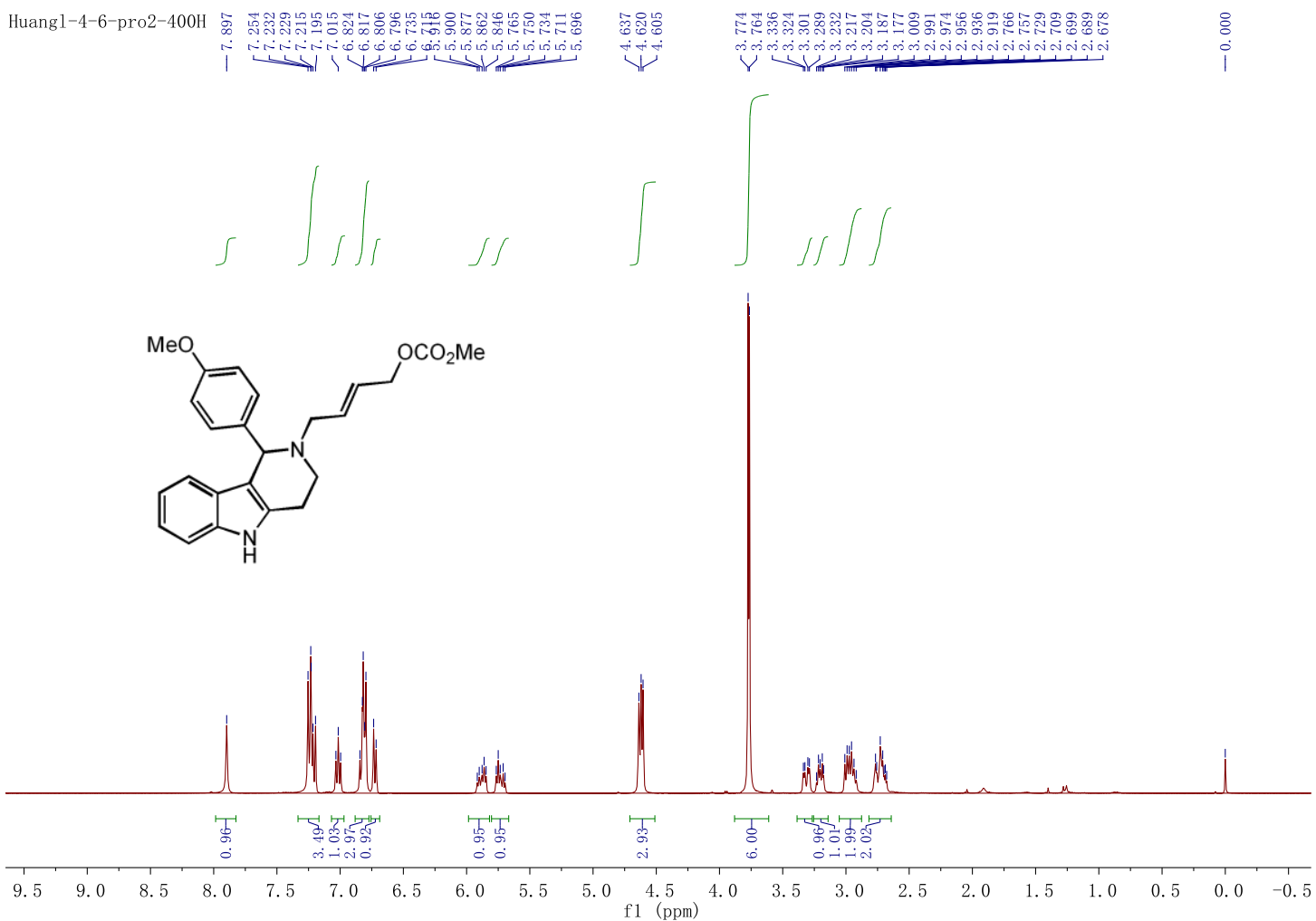



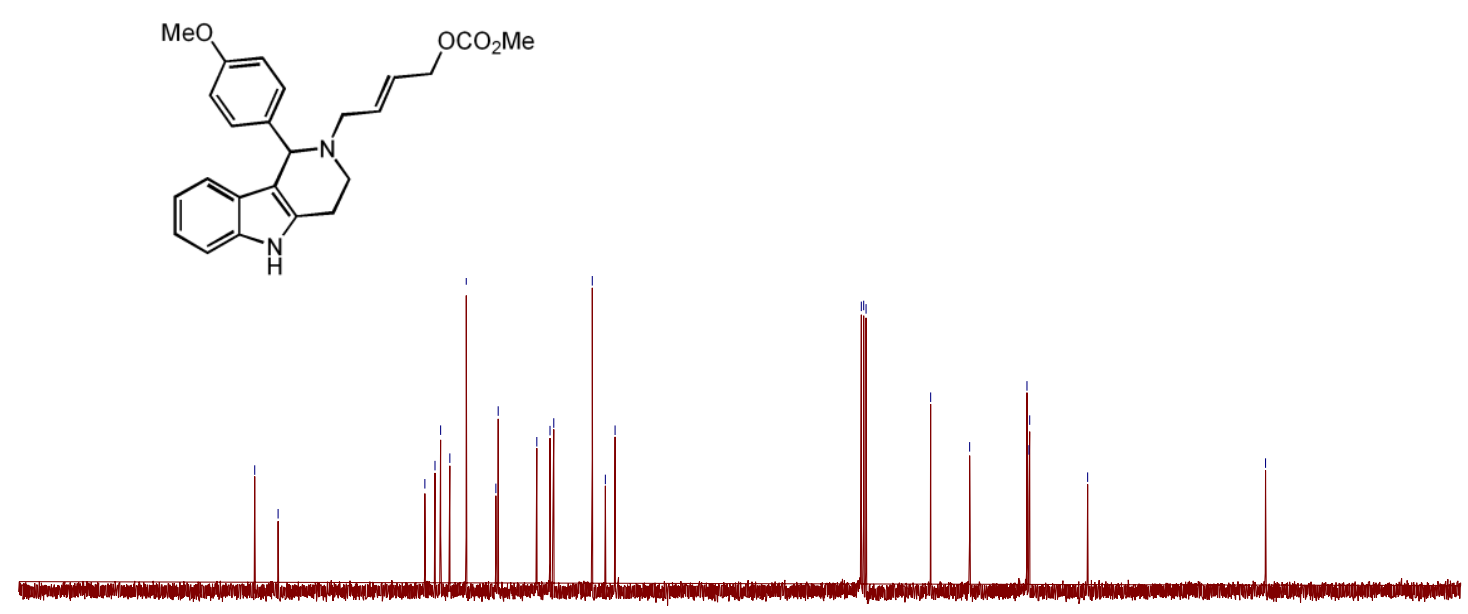

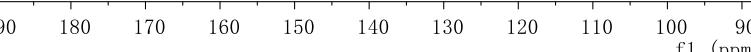

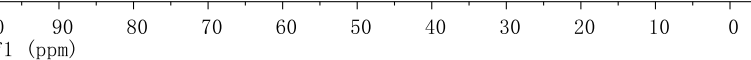

\section{NMR Spectra of $\mathbf{1 b}$}

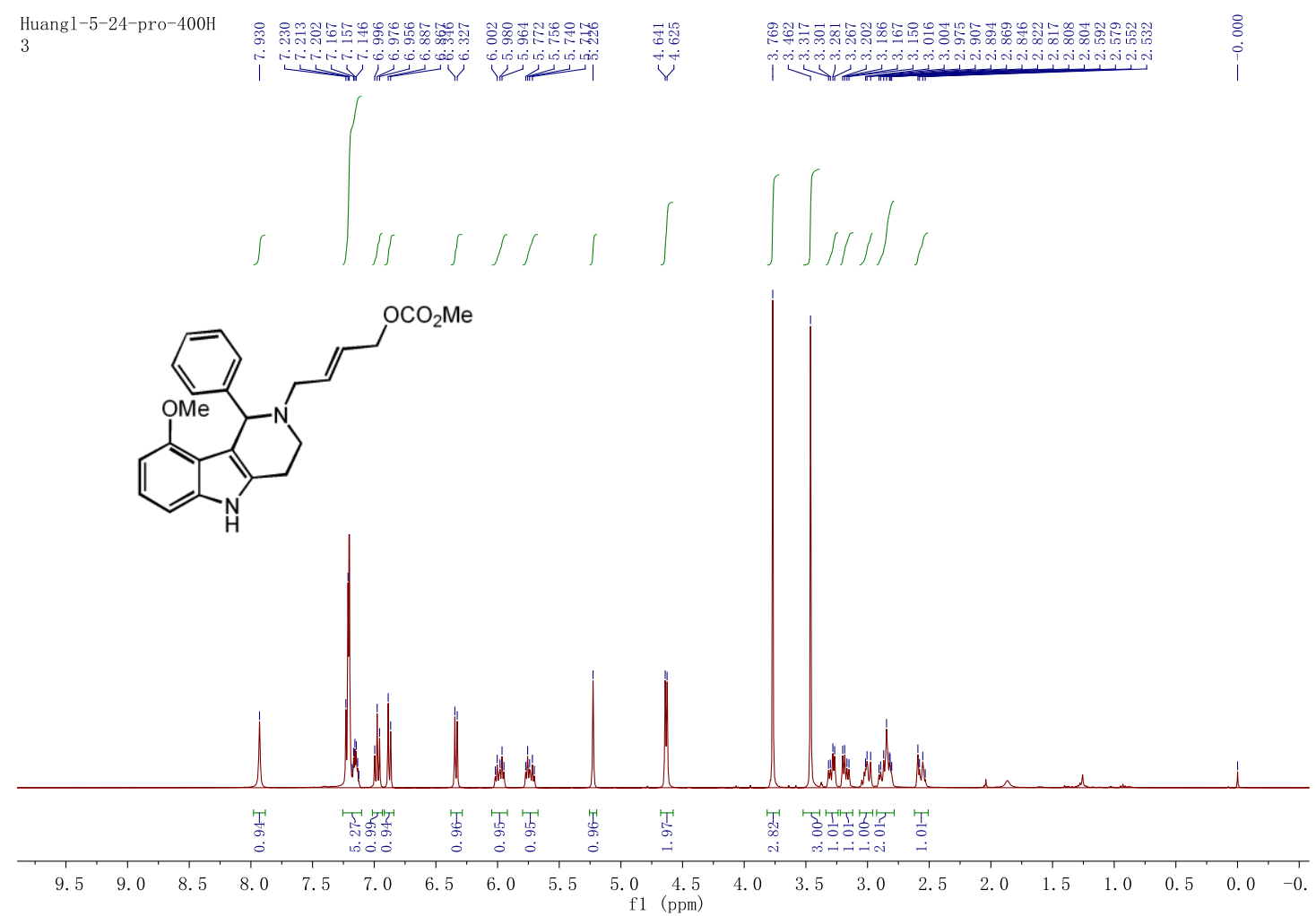



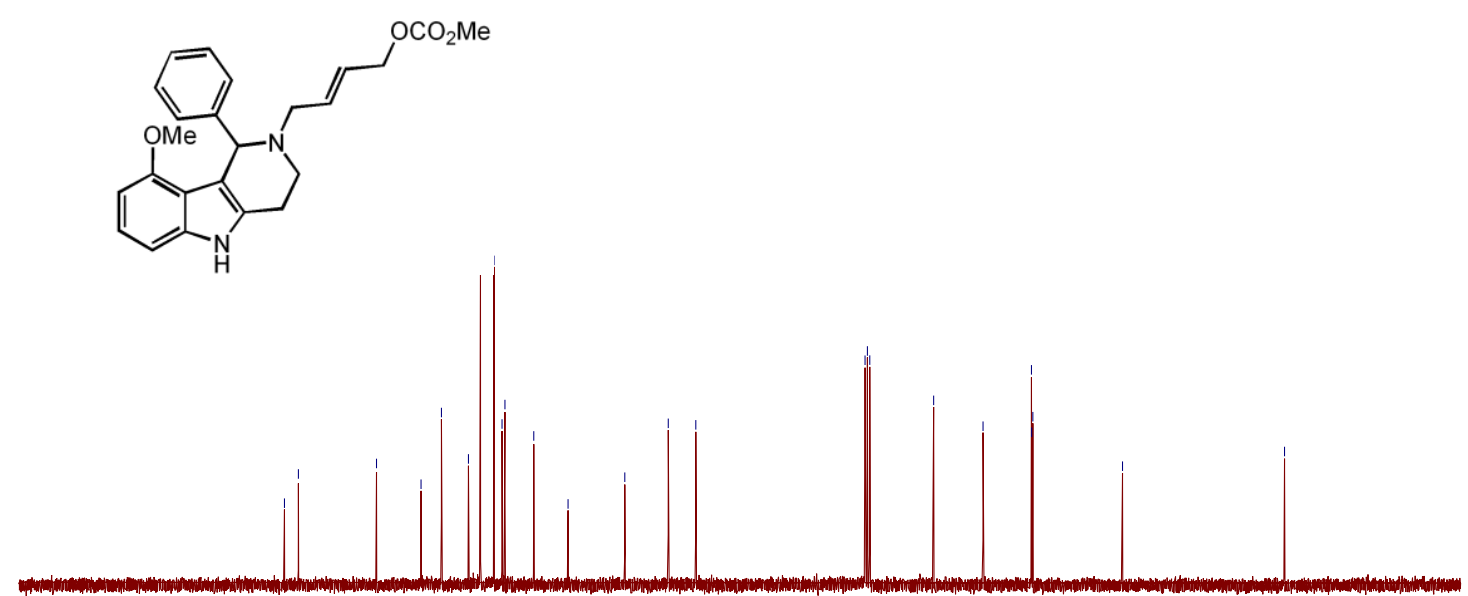

$\begin{array}{rrrrrrrrrr}190 & 180 & 170 & 160 & 150 & 140 & 130 & 120 & 110 & 100 \\ \mathrm{f} 1 & \begin{array}{r}9 \\ (\mathrm{ppm})\end{array}\end{array}$

\section{NMR Spectra of 1c}

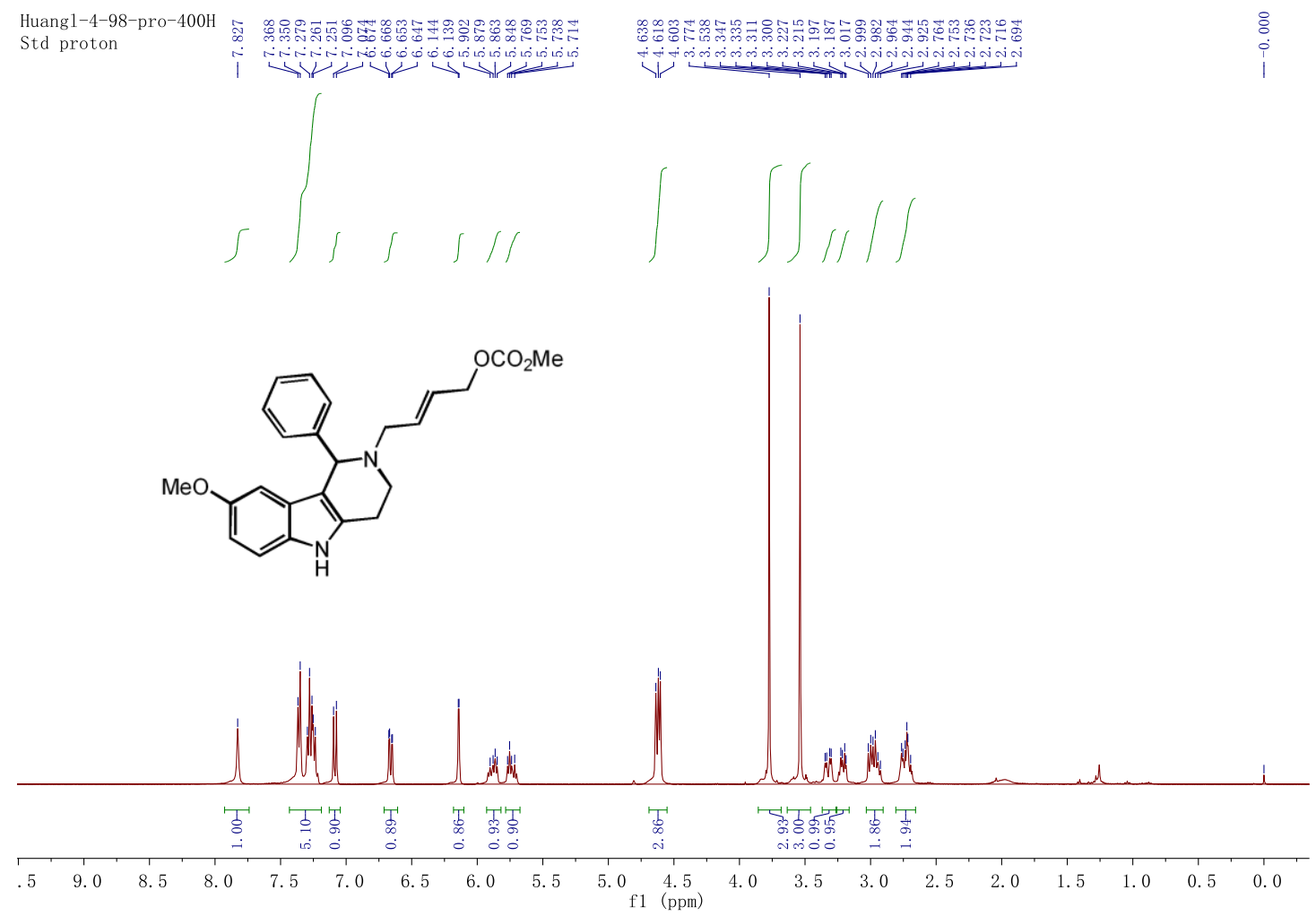




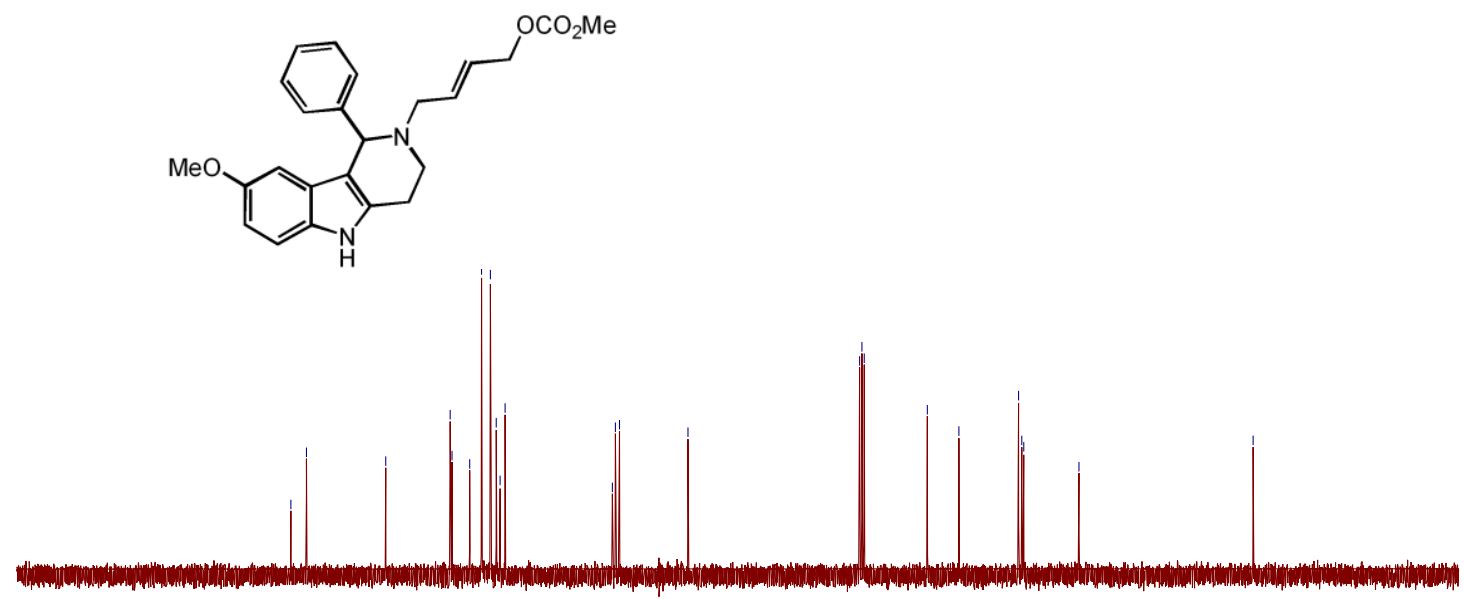

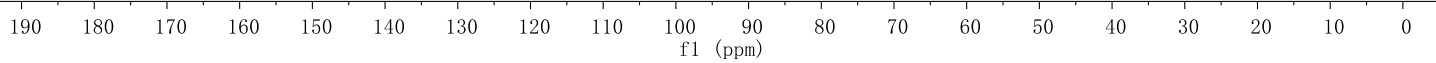

\section{NMR Spectra of 1d}

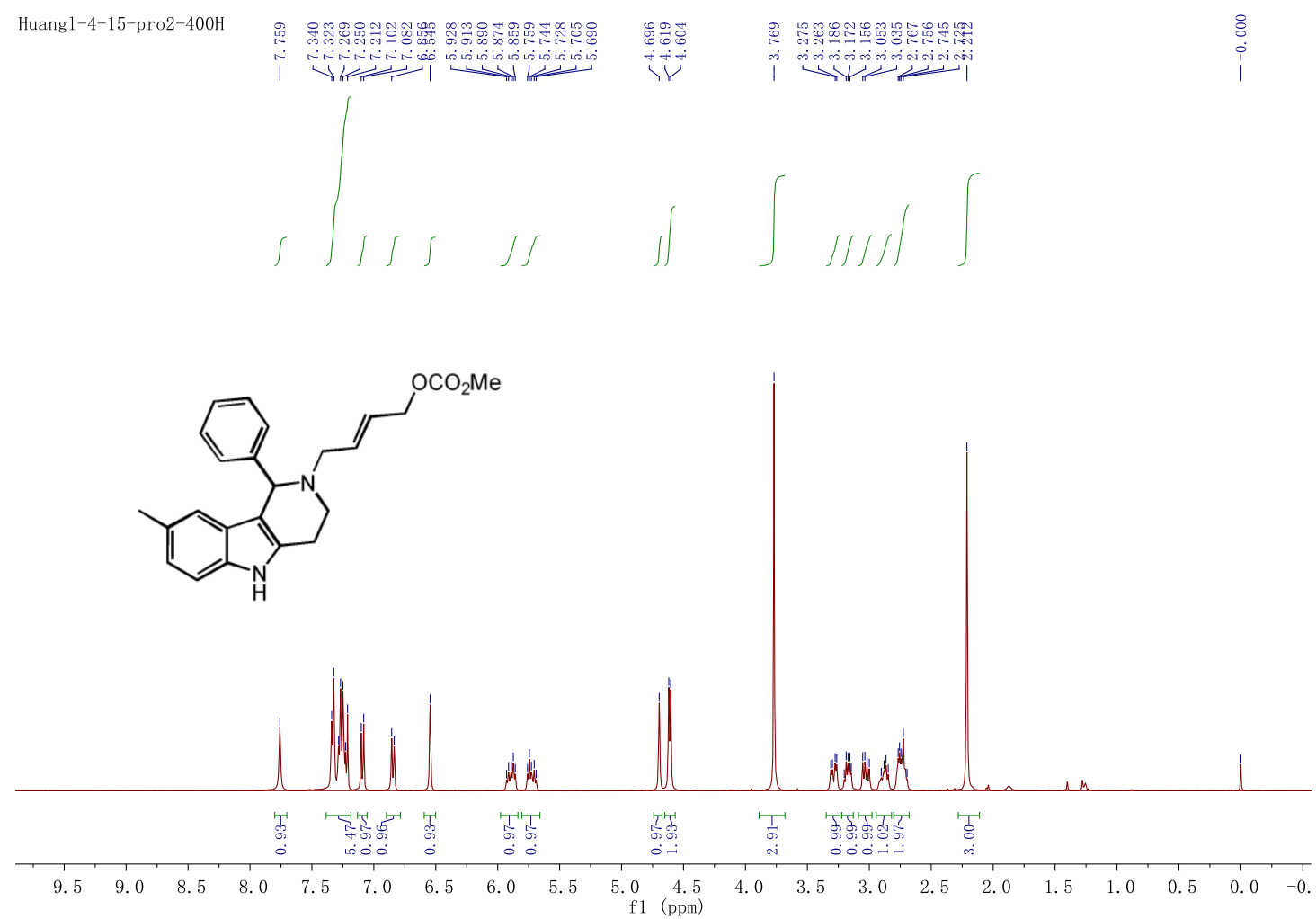



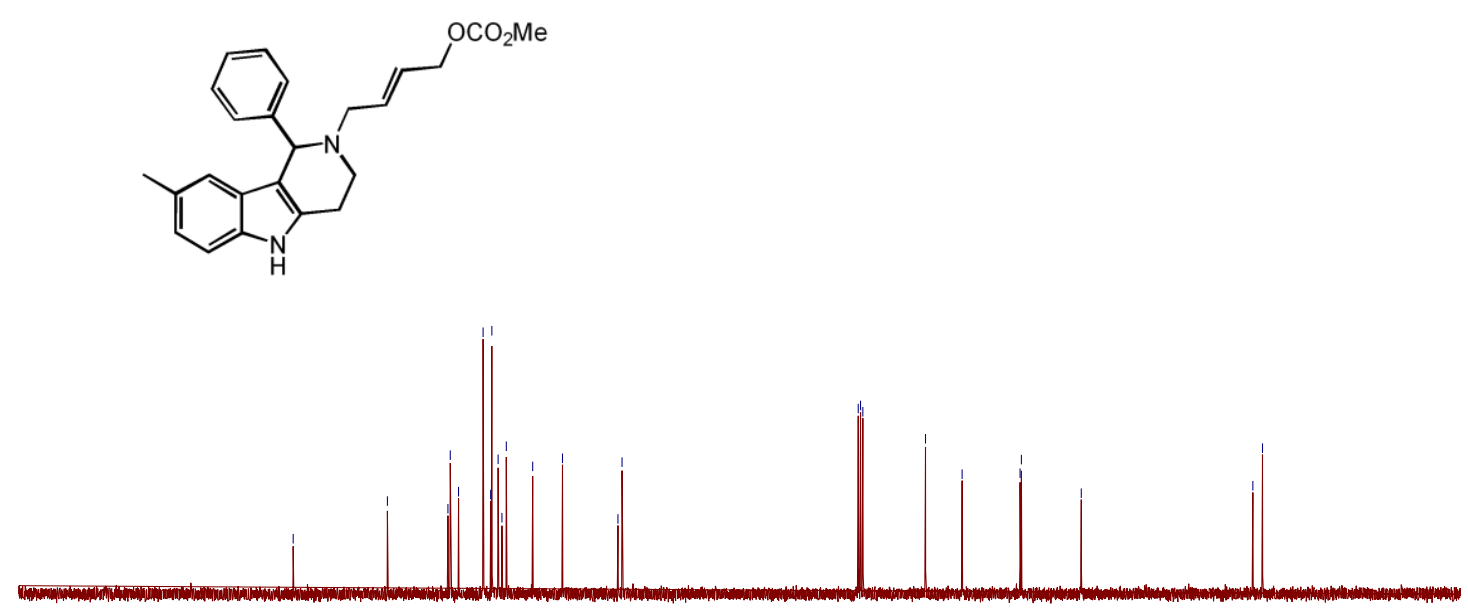

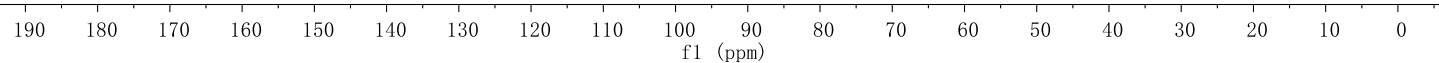

\section{NMR Spectra of 1e}

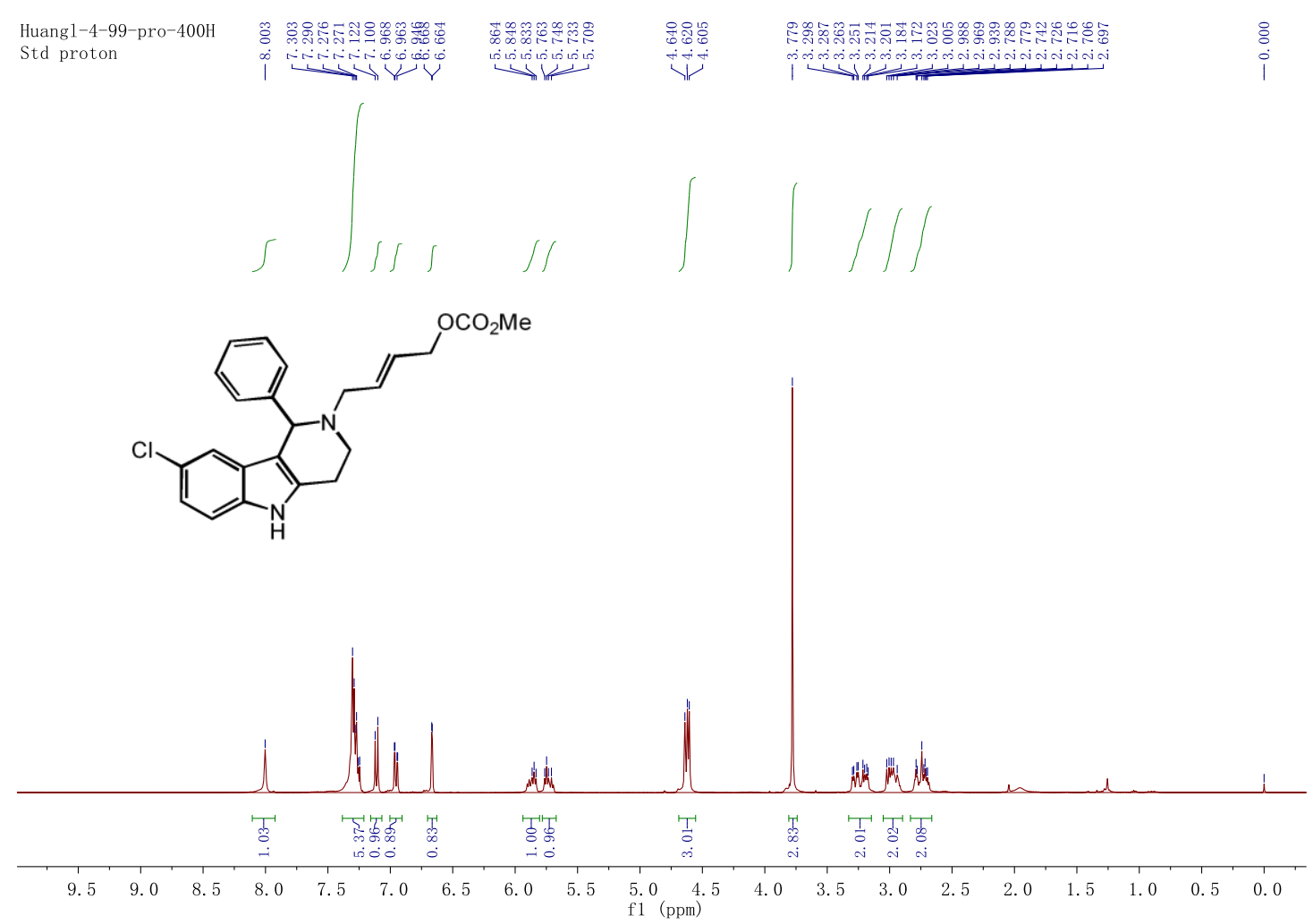



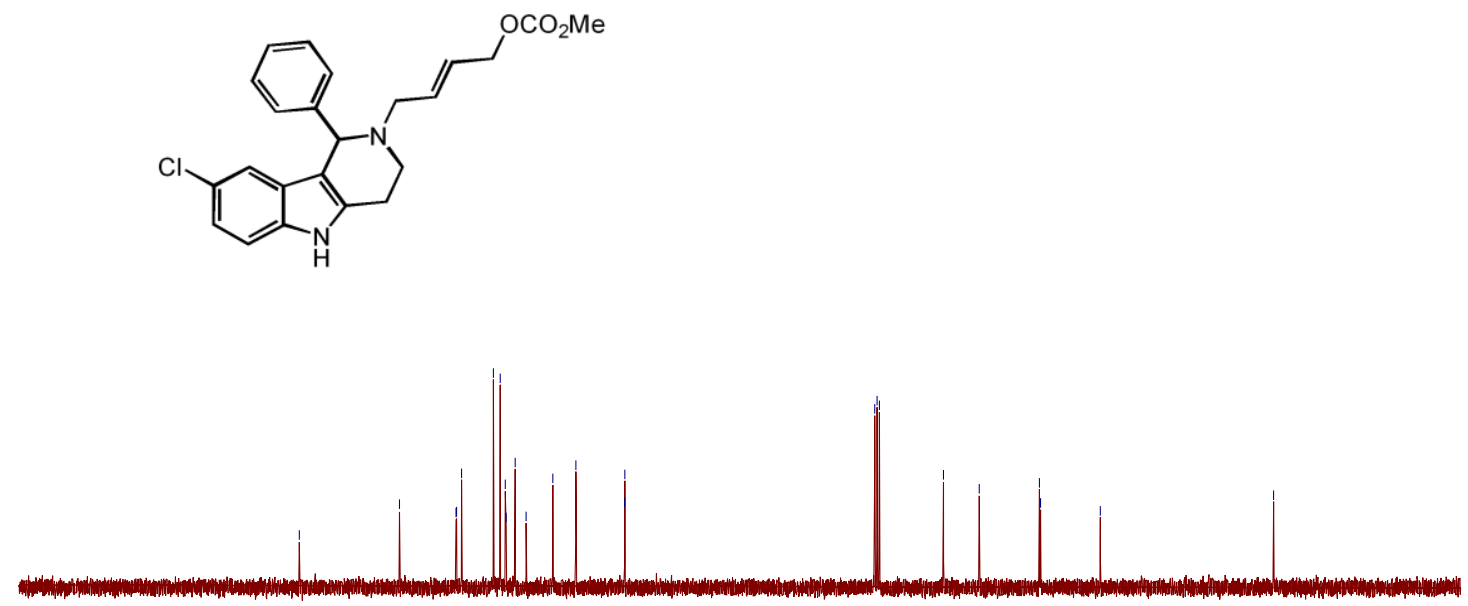

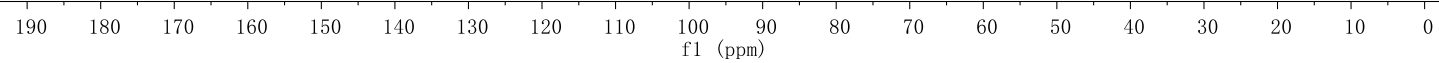

\section{NMR Spectra of $\mathbf{1 f}$}

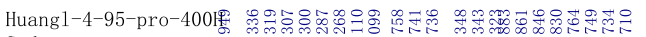
Std proton

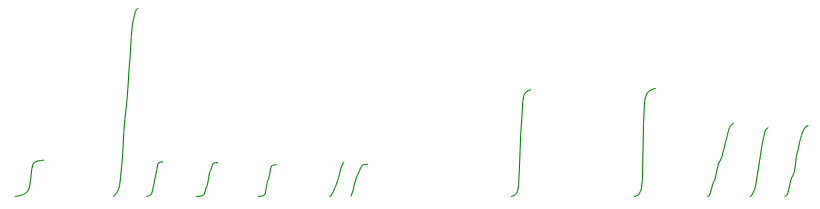<smiles>COCC=CCN1CCc2[nH]c3ccc(F)cc3c2C1c1ccccc1</smiles>

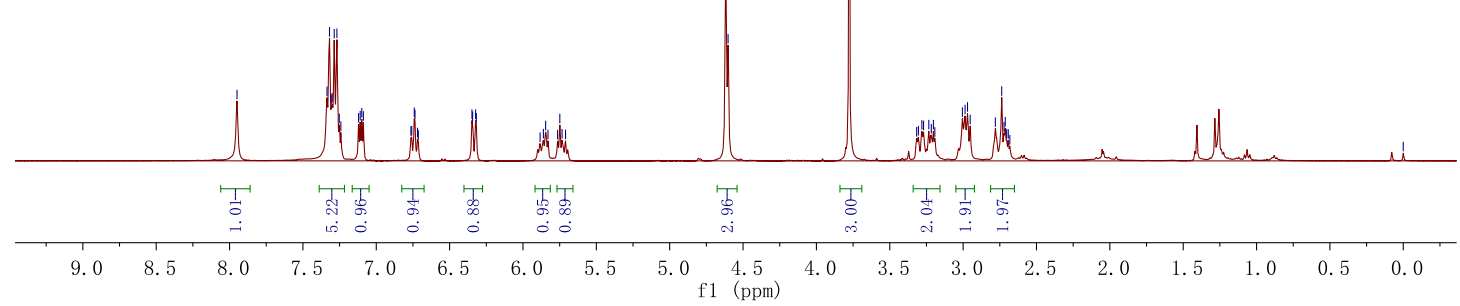



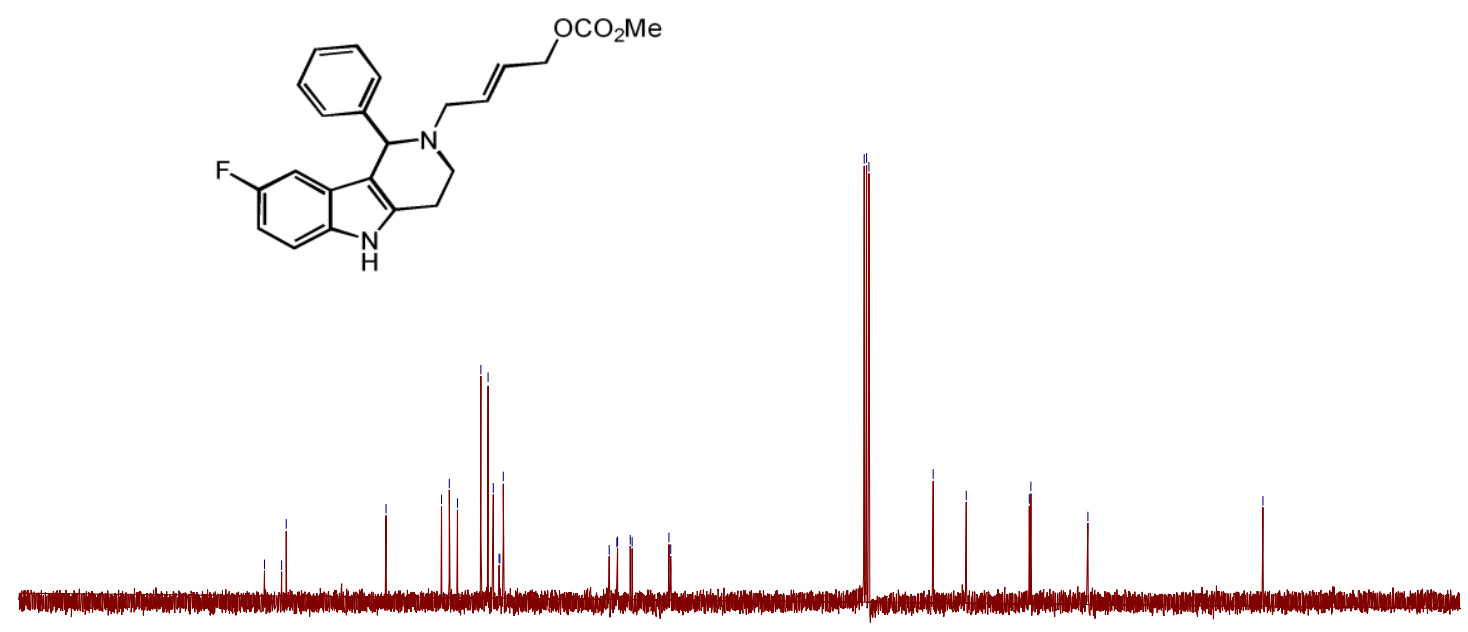

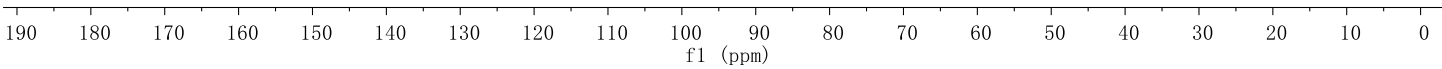

Huang 1-4-95-pro-400

Std Fluorine

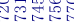

ga<smiles>COCC=CCN1CCc2[nH]c3ccc(F)cc3c2C1c1ccccc1</smiles>

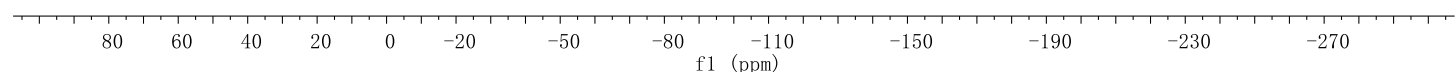

NMR Spectra of $1 \mathrm{~g}$ 
<smiles>C=CC(=C)CC</smiles>

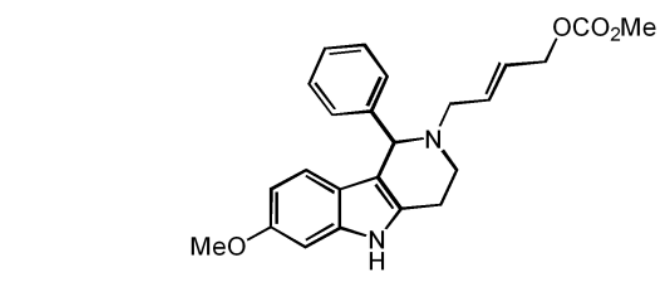

Huang 1-4-35-pro-400
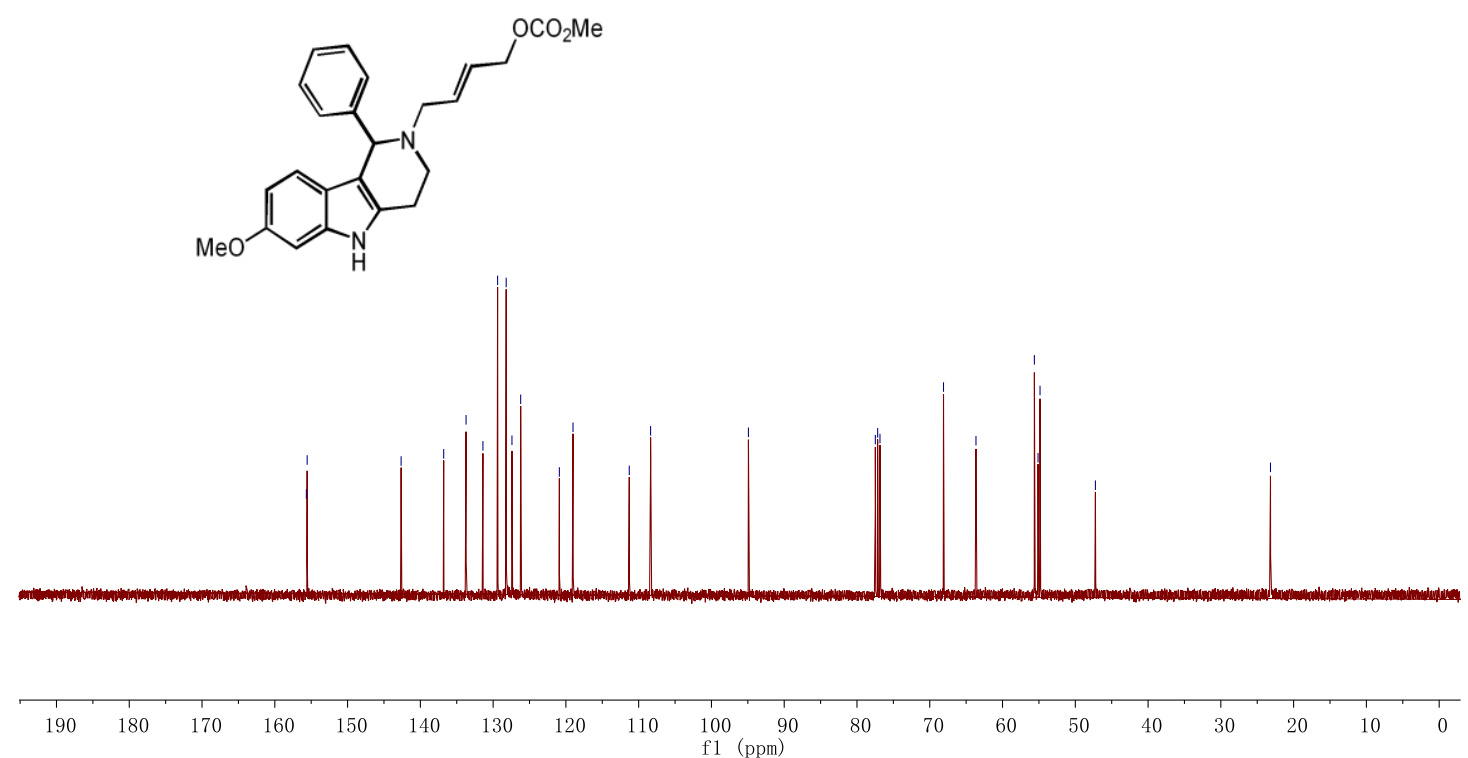

NMR Spectra of $\mathbf{1 h}$ 

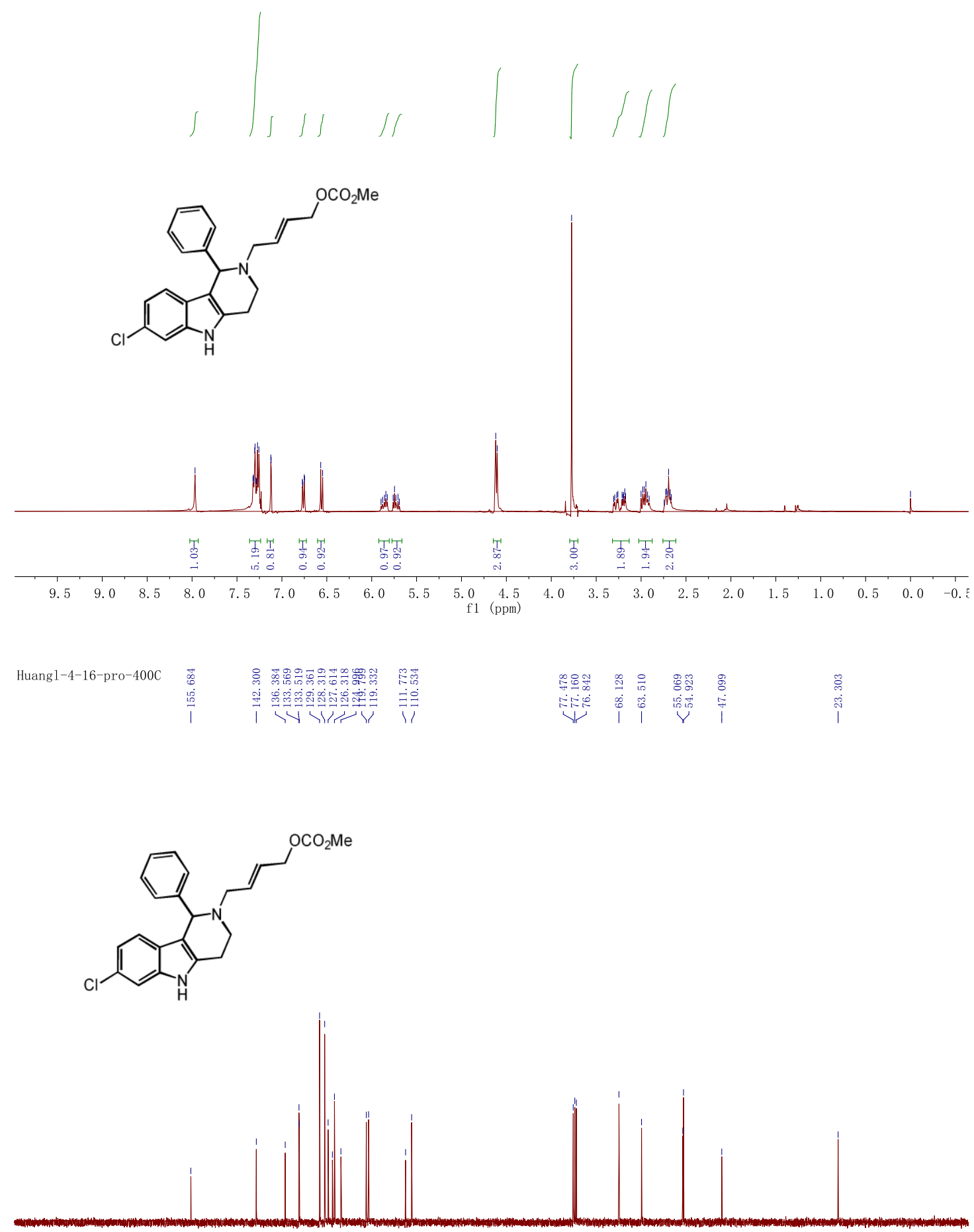

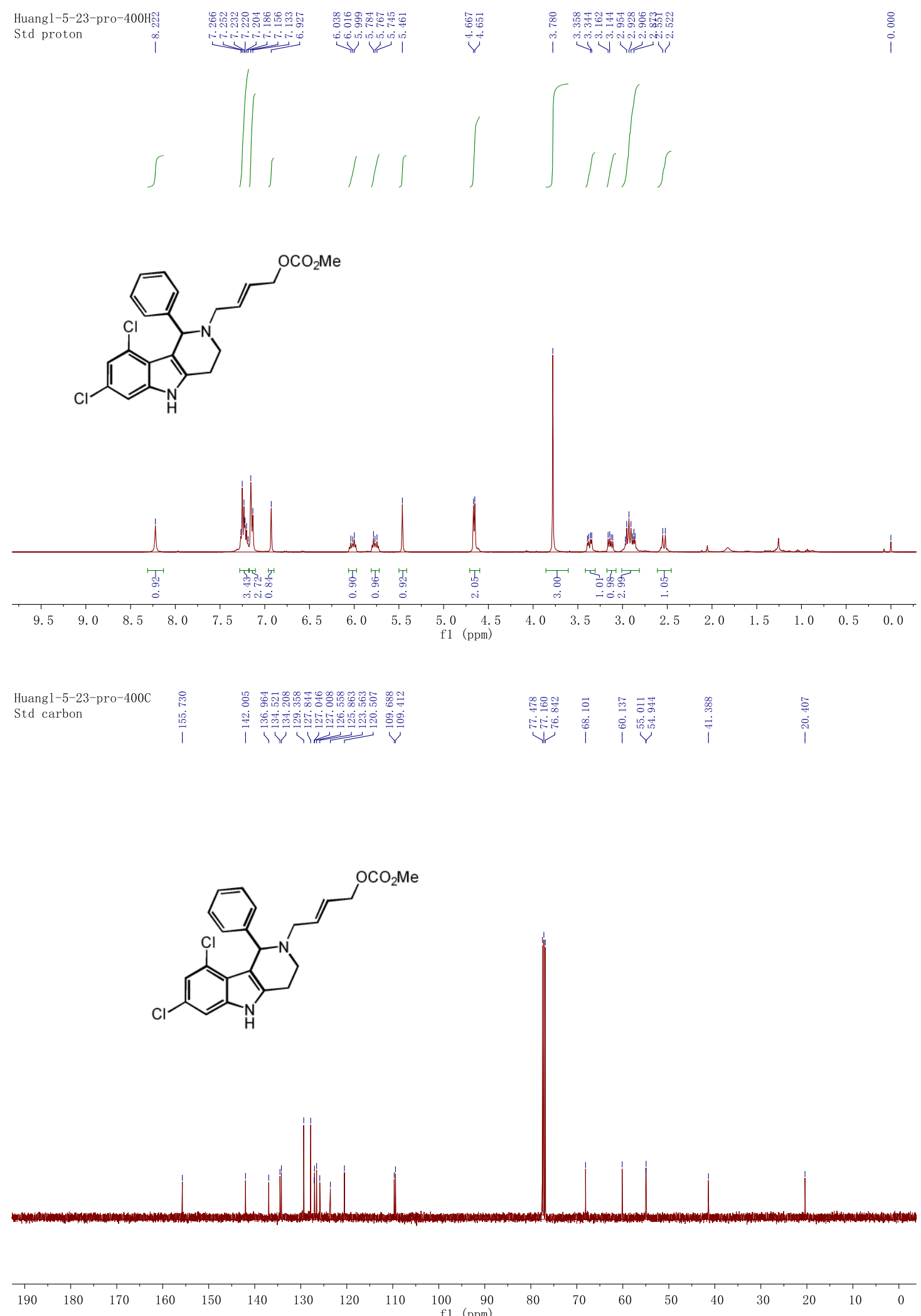

NMR Spectra of $\mathbf{1 j}$ 

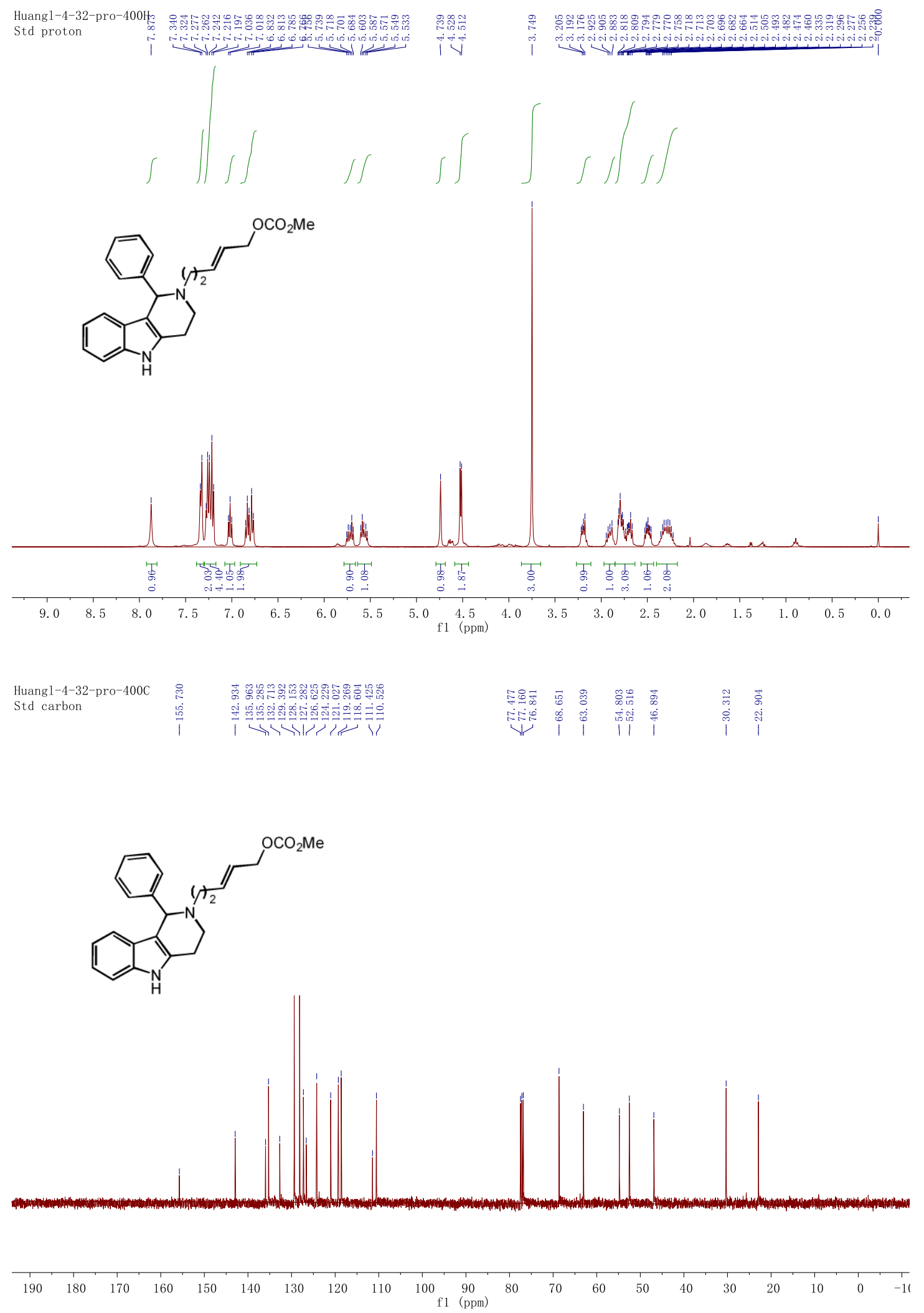

NMR Spectra of $\mathbf{1 k}$ 

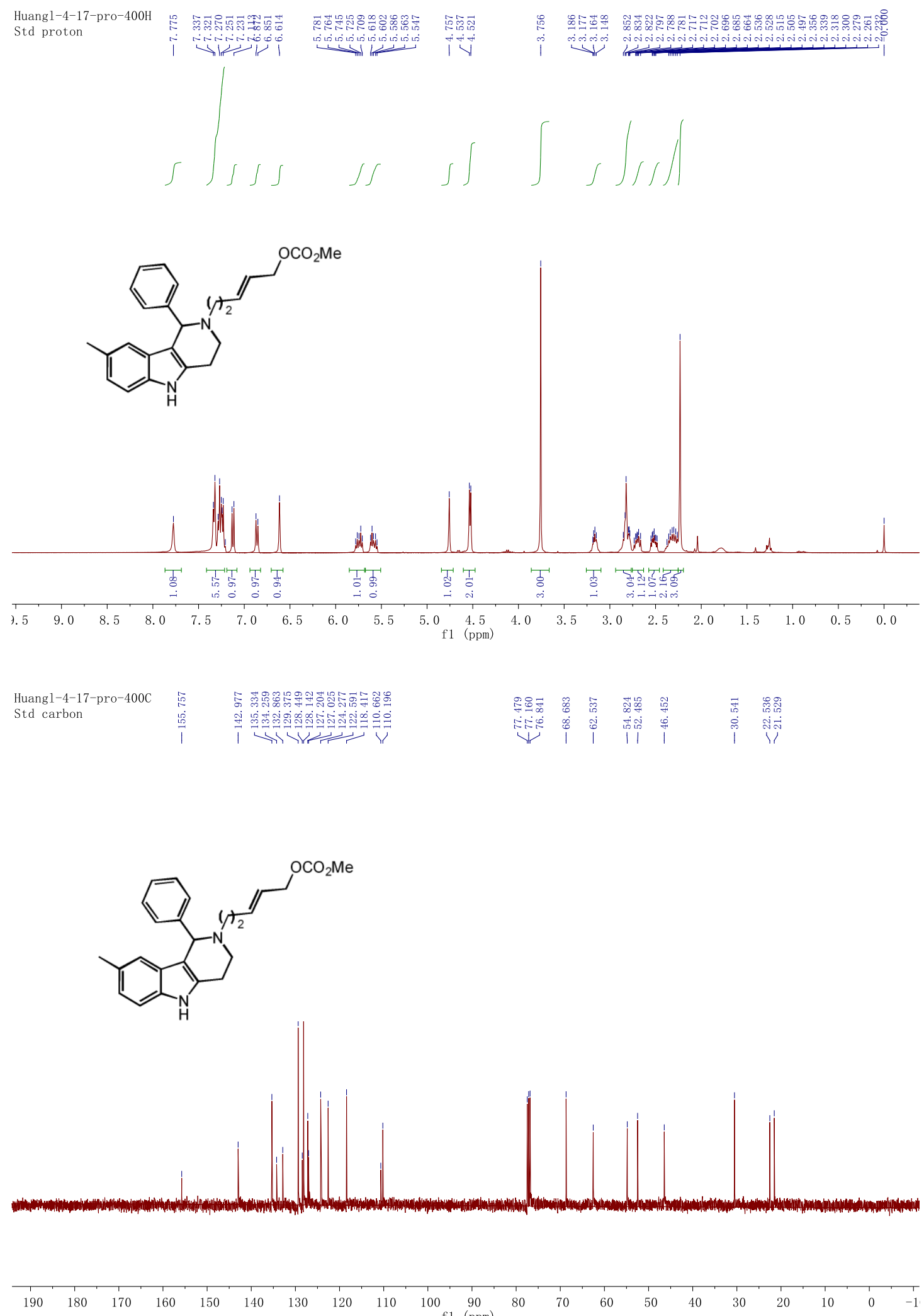

NMR Spectra of $\mathbf{1 l}$ 


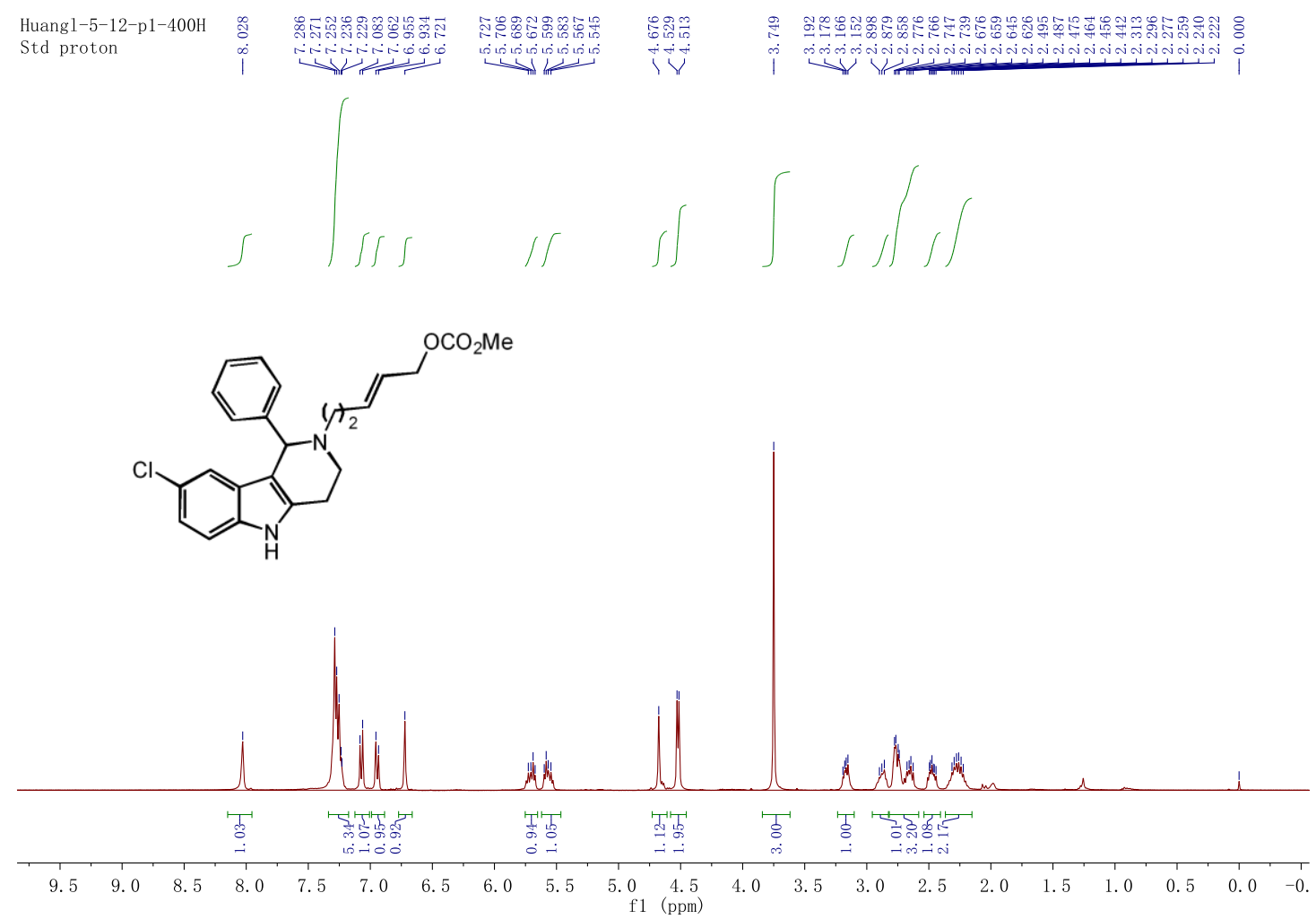

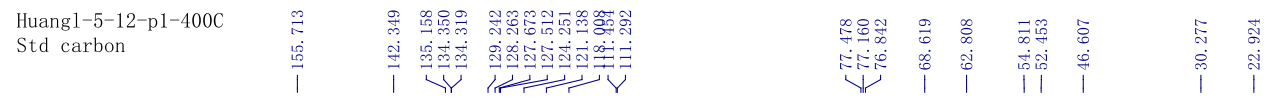<smiles>COCC=CCN1CCc2[nH]c3ccc(Cl)cc3c2C1c1ccccc1</smiles>

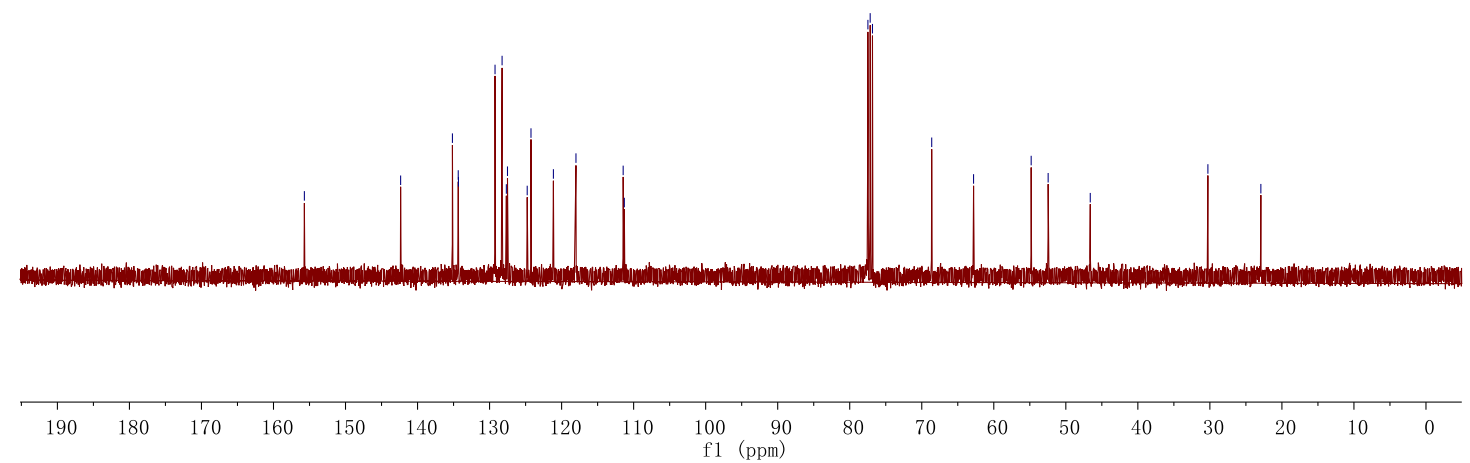

NMR Spectra of $\mathbf{1 m}$ 

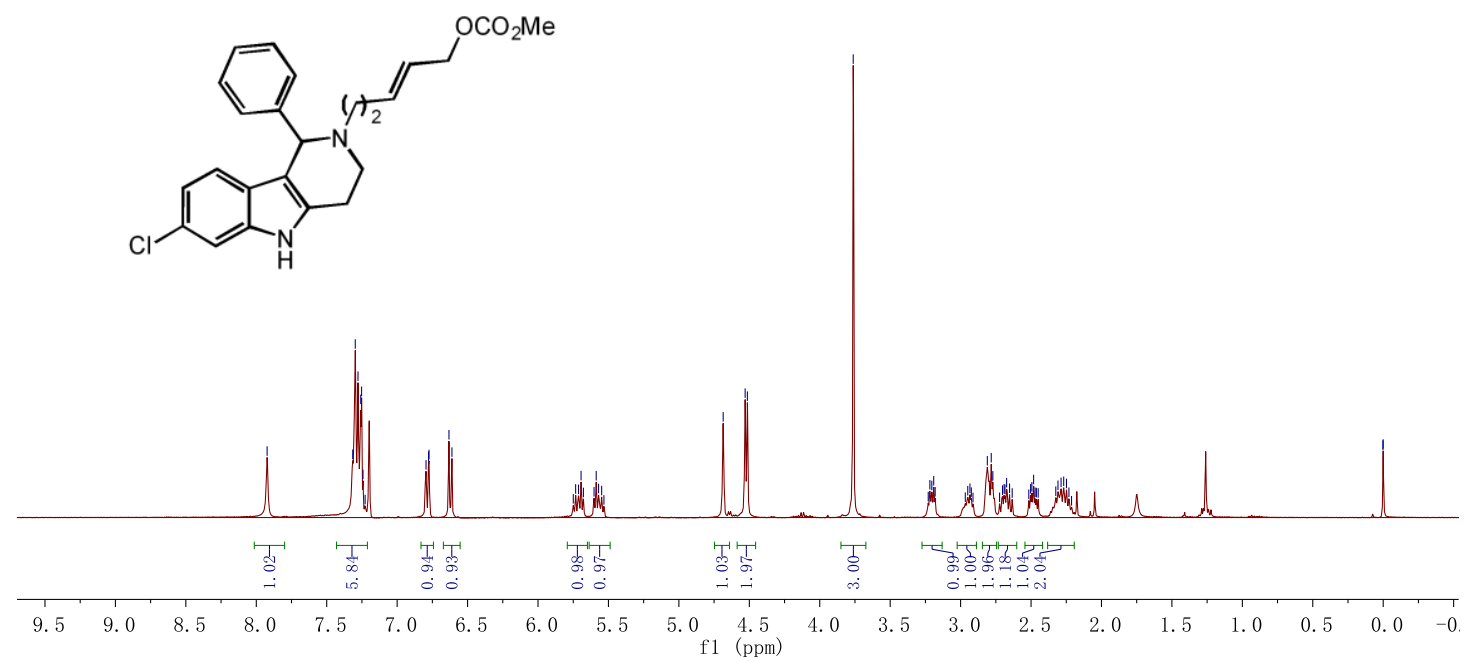<smiles>COCC=CCN1CCc2[nH]c3cc(Cl)ccc3c2C1c1ccccc1</smiles>

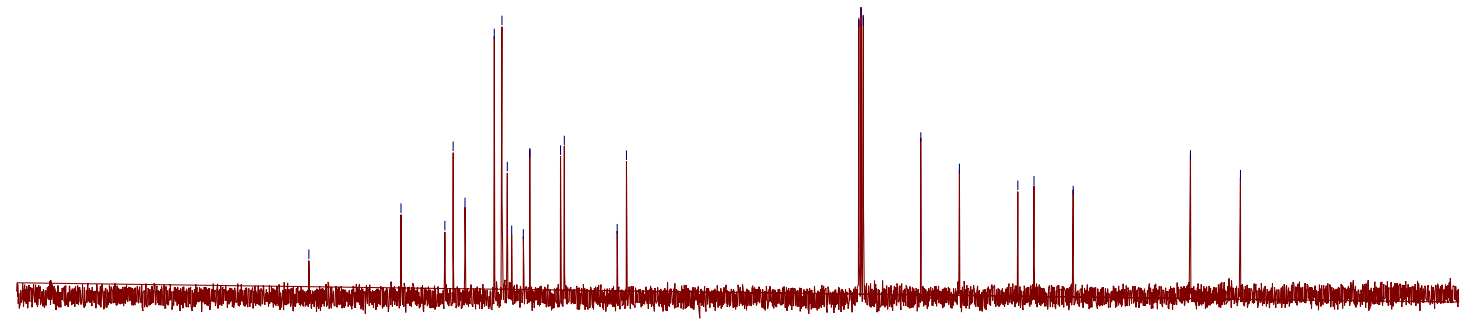

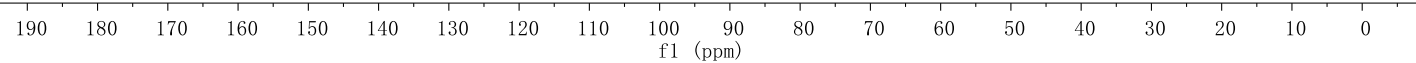

NMR Spectra of $\mathbf{1 n}$ 

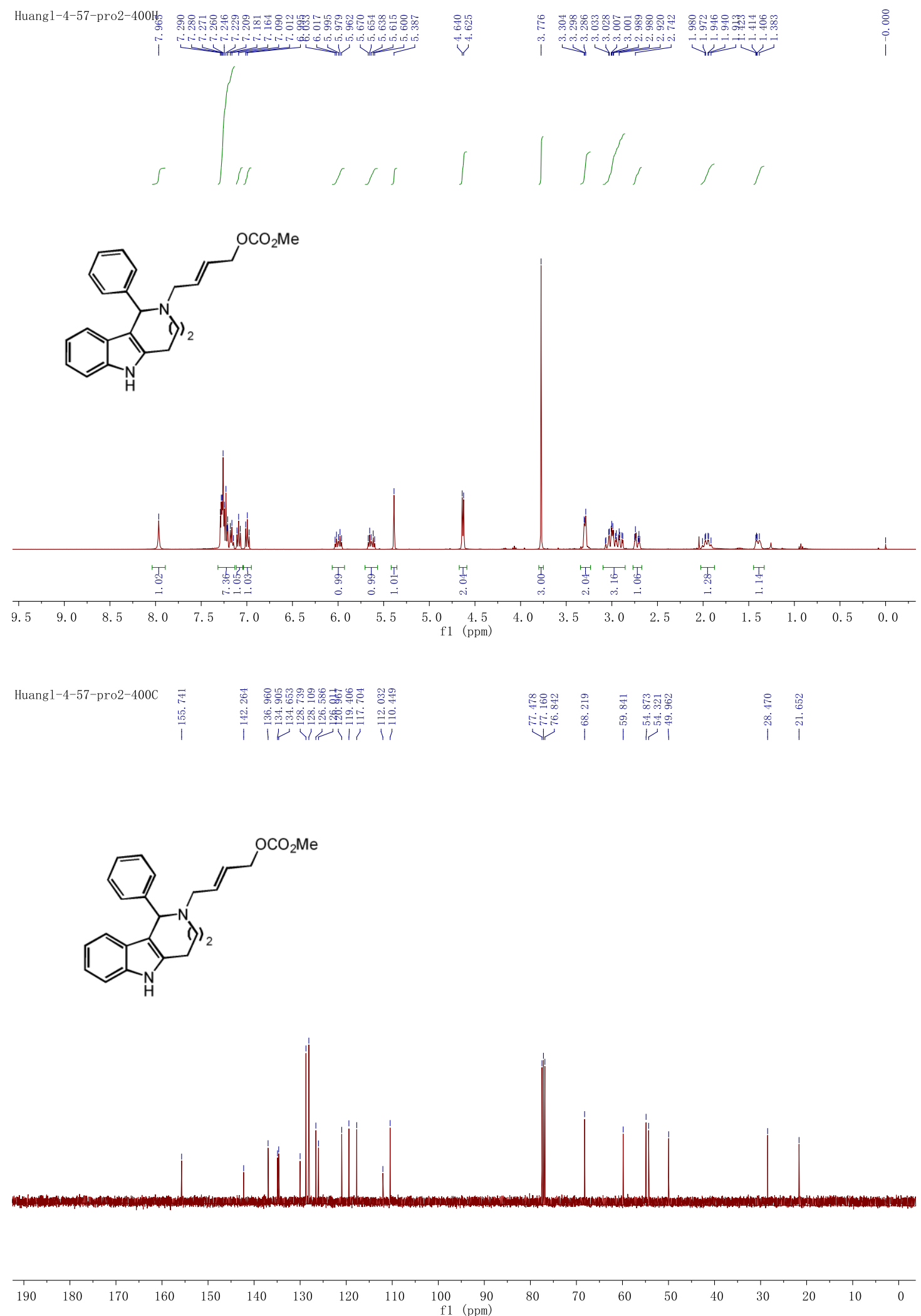

NMR Spectra of $\mathbf{1 o}$ 


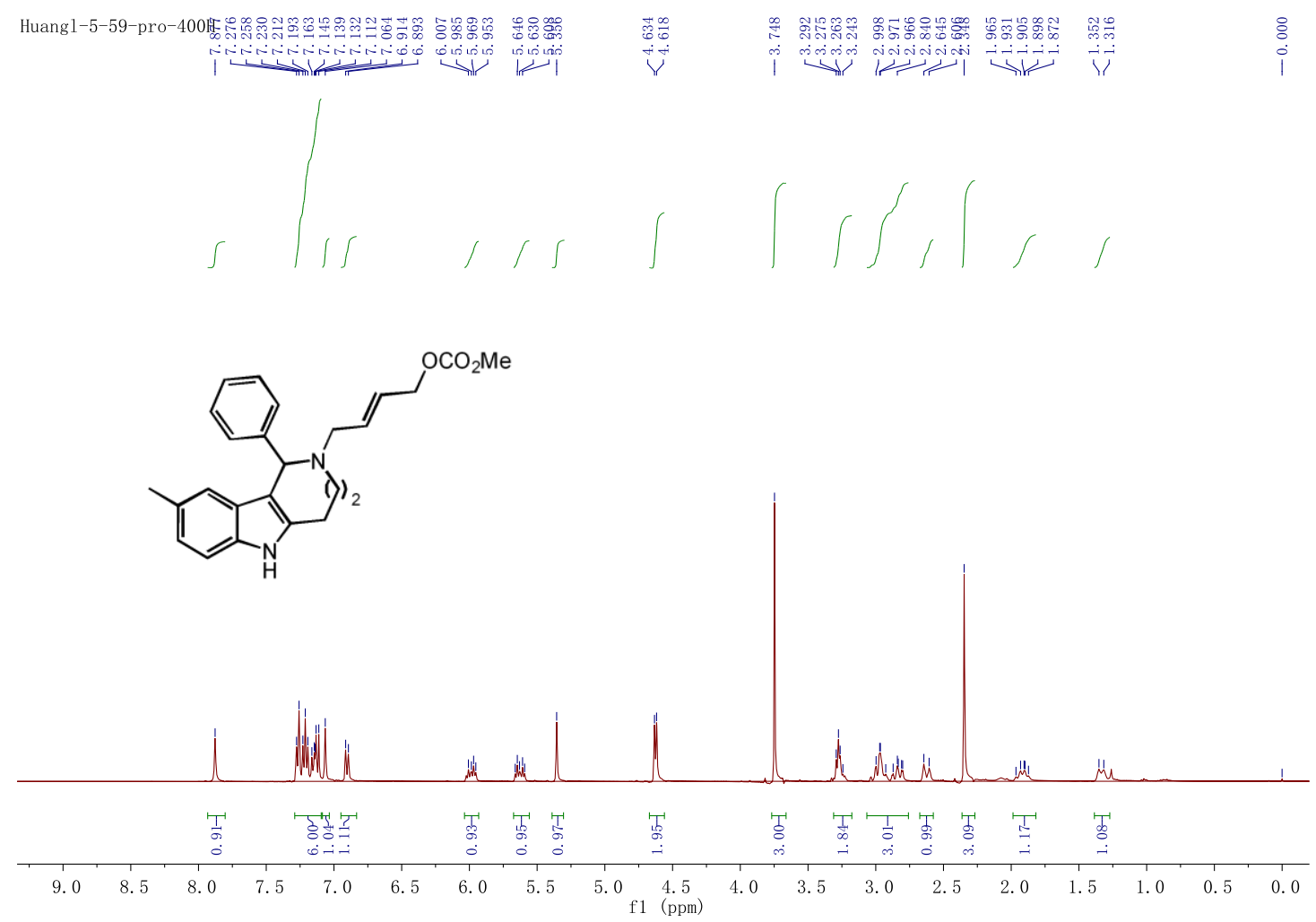

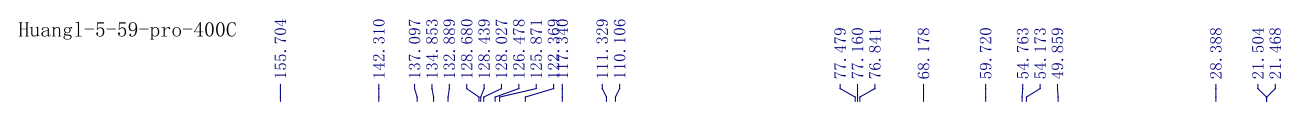
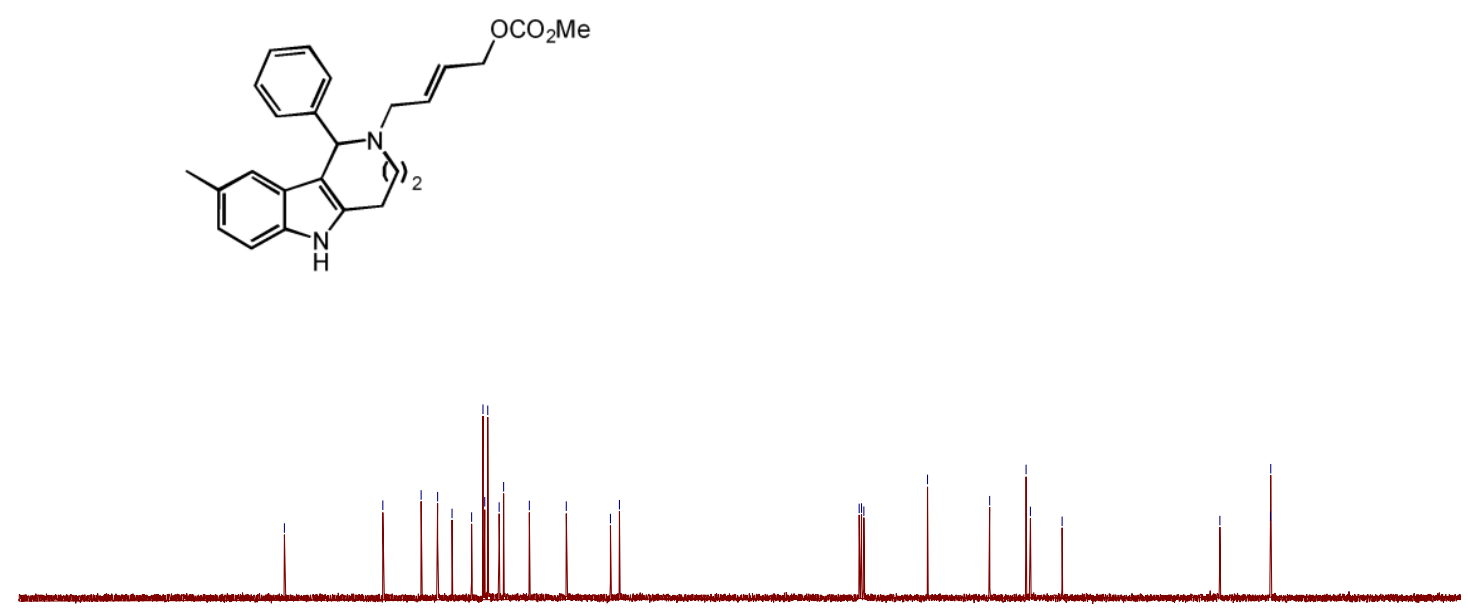

NMR Spectra of 1p 

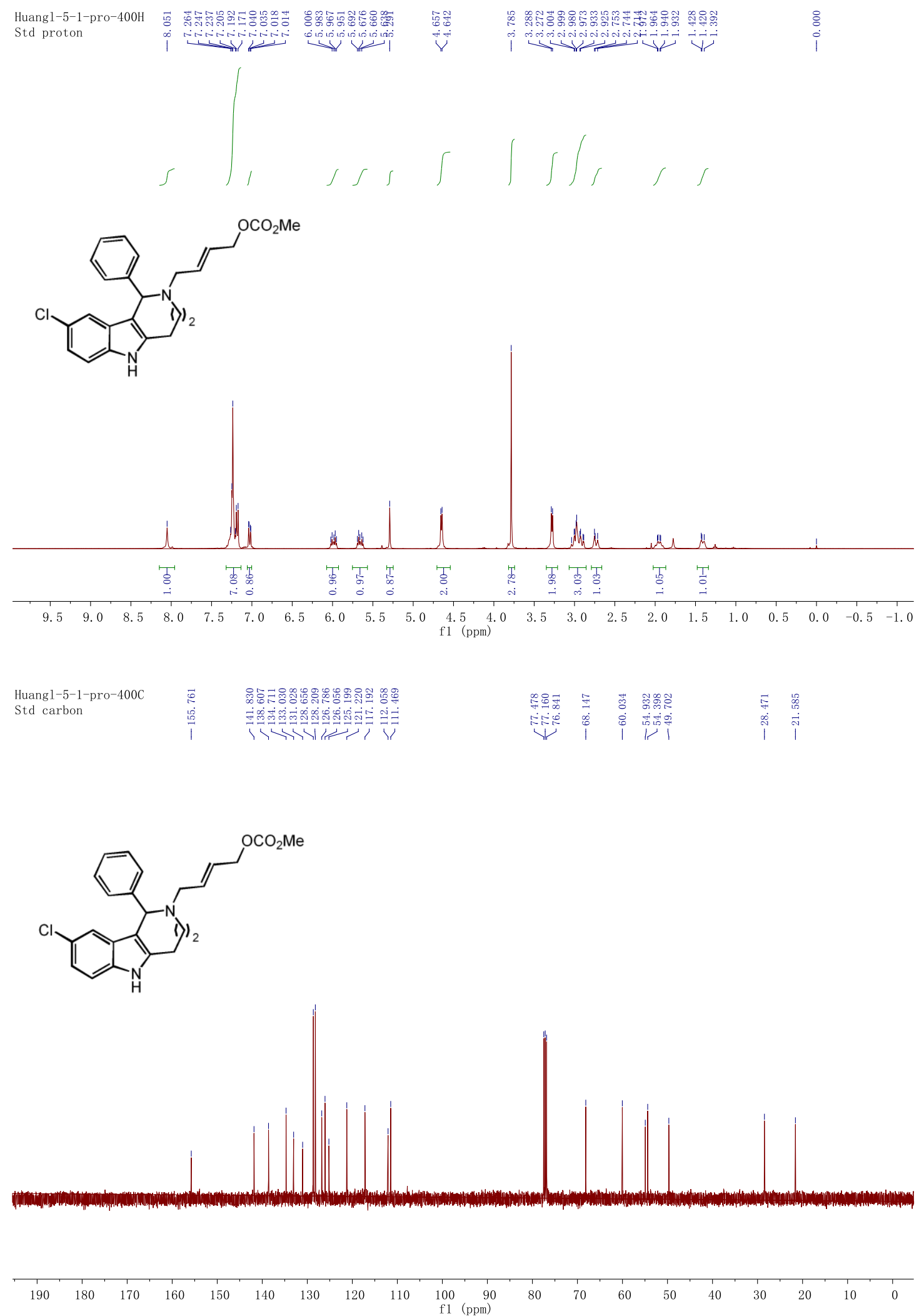

NMR Spectra of 1q 

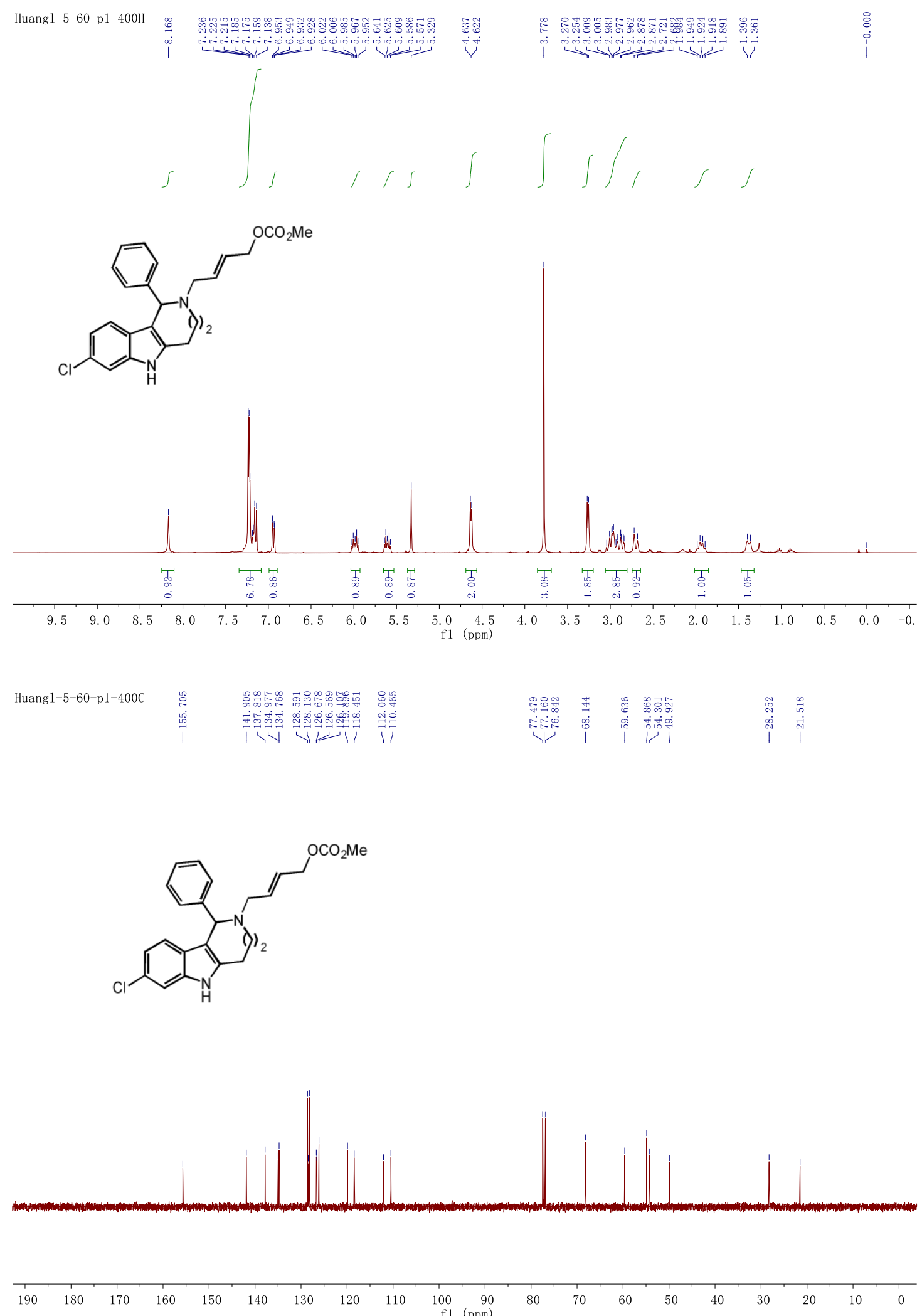

NMR Spectra of $\mathbf{1 r}$ 


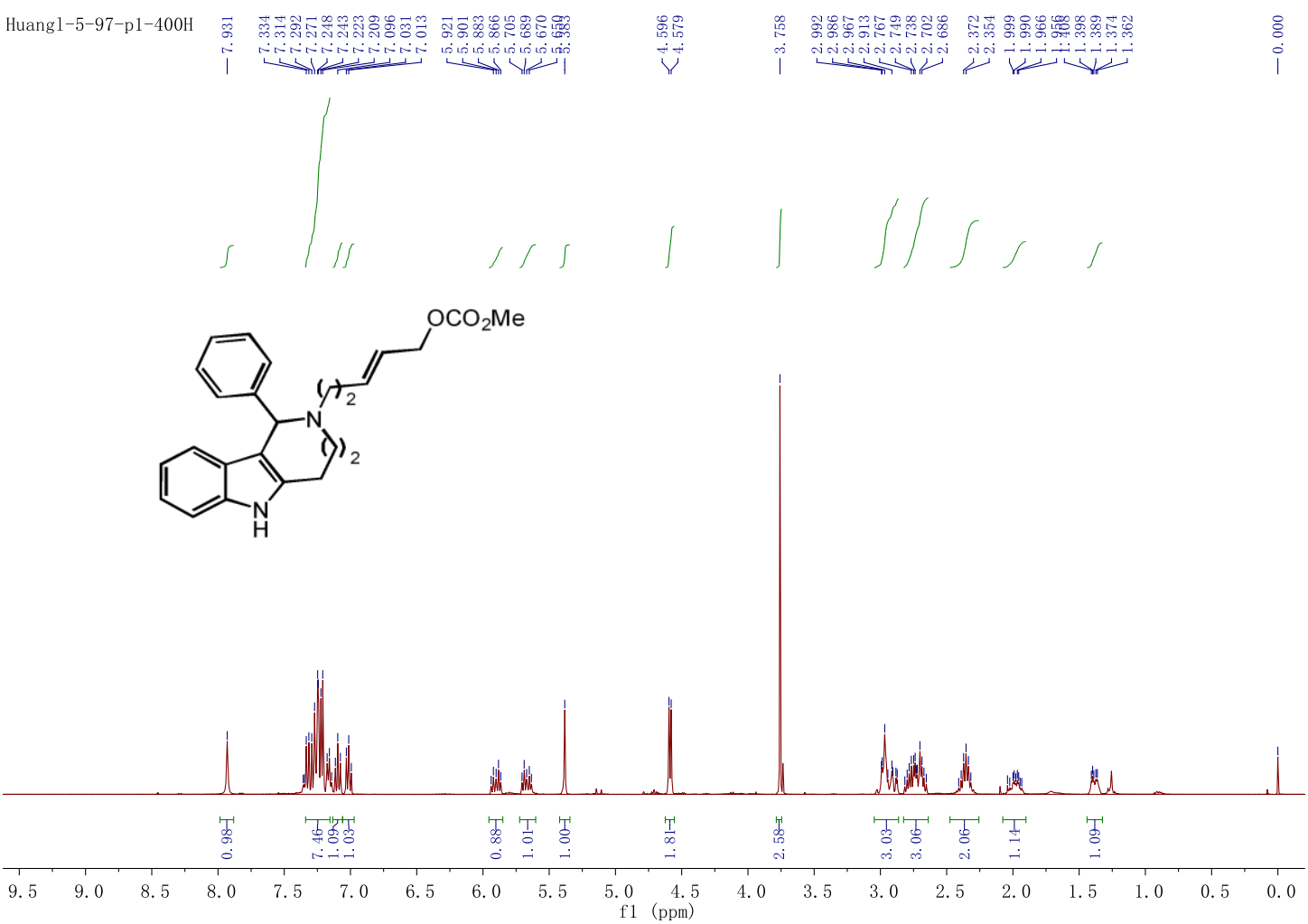

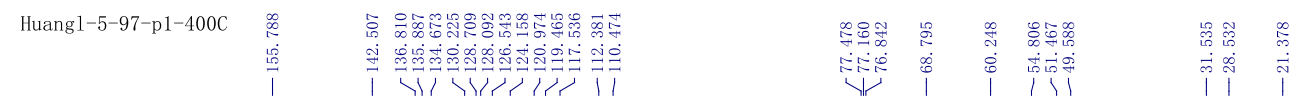

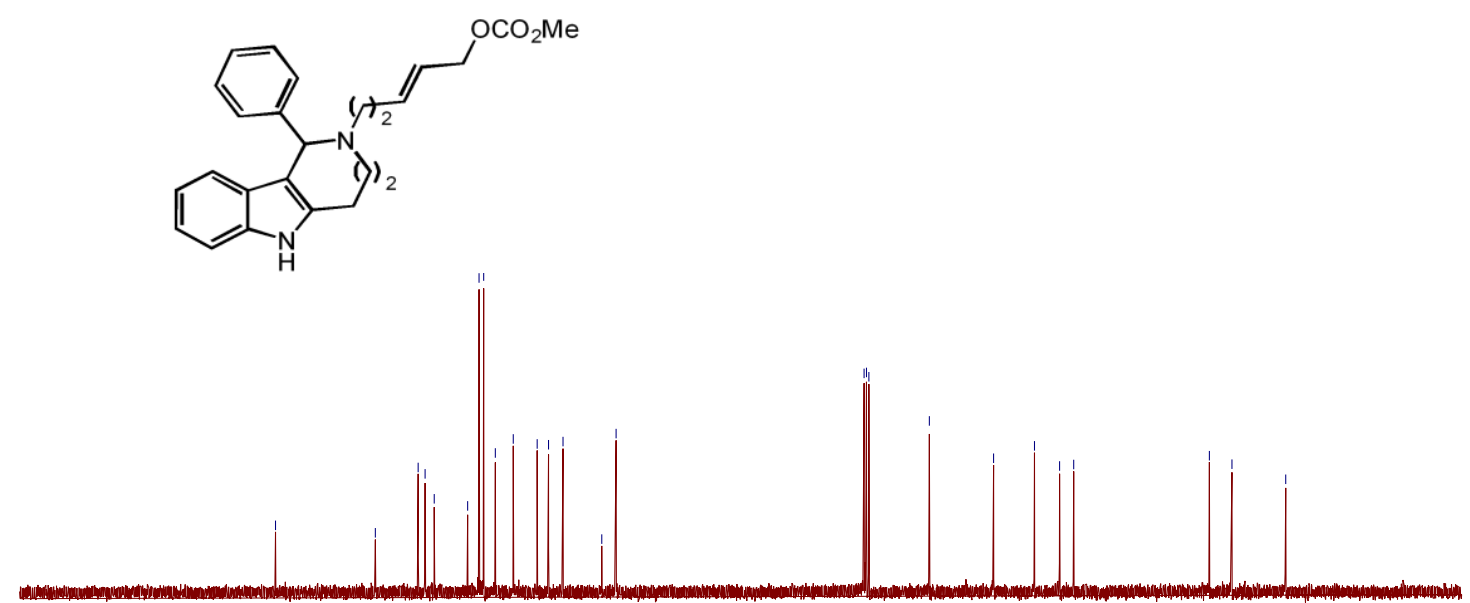

NMR Spectra of $1 \mathbf{s}$ 

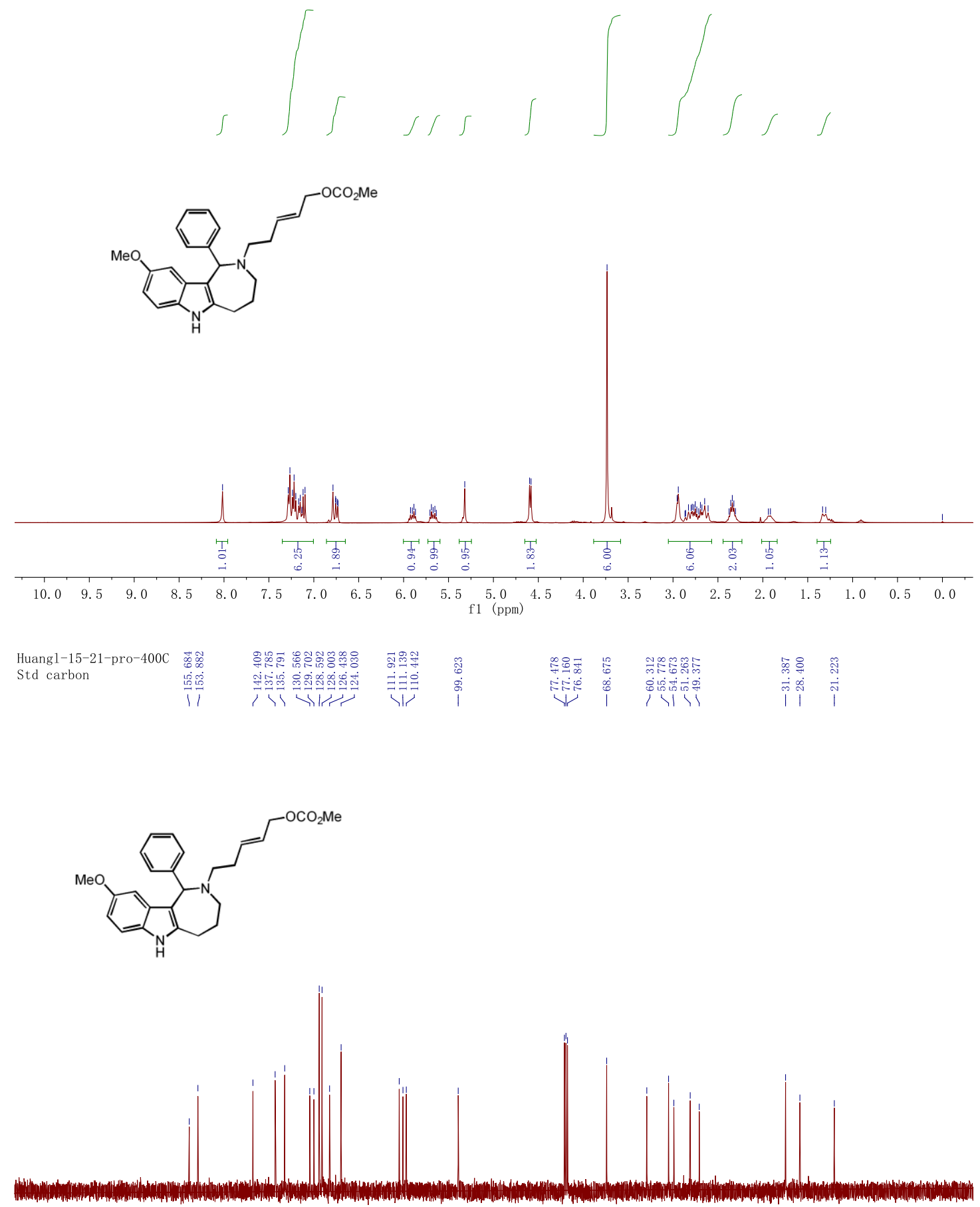

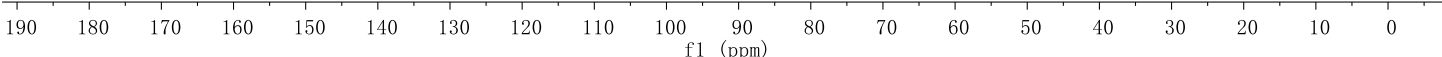

NMR Spectra of $\mathbf{1 t}$ 


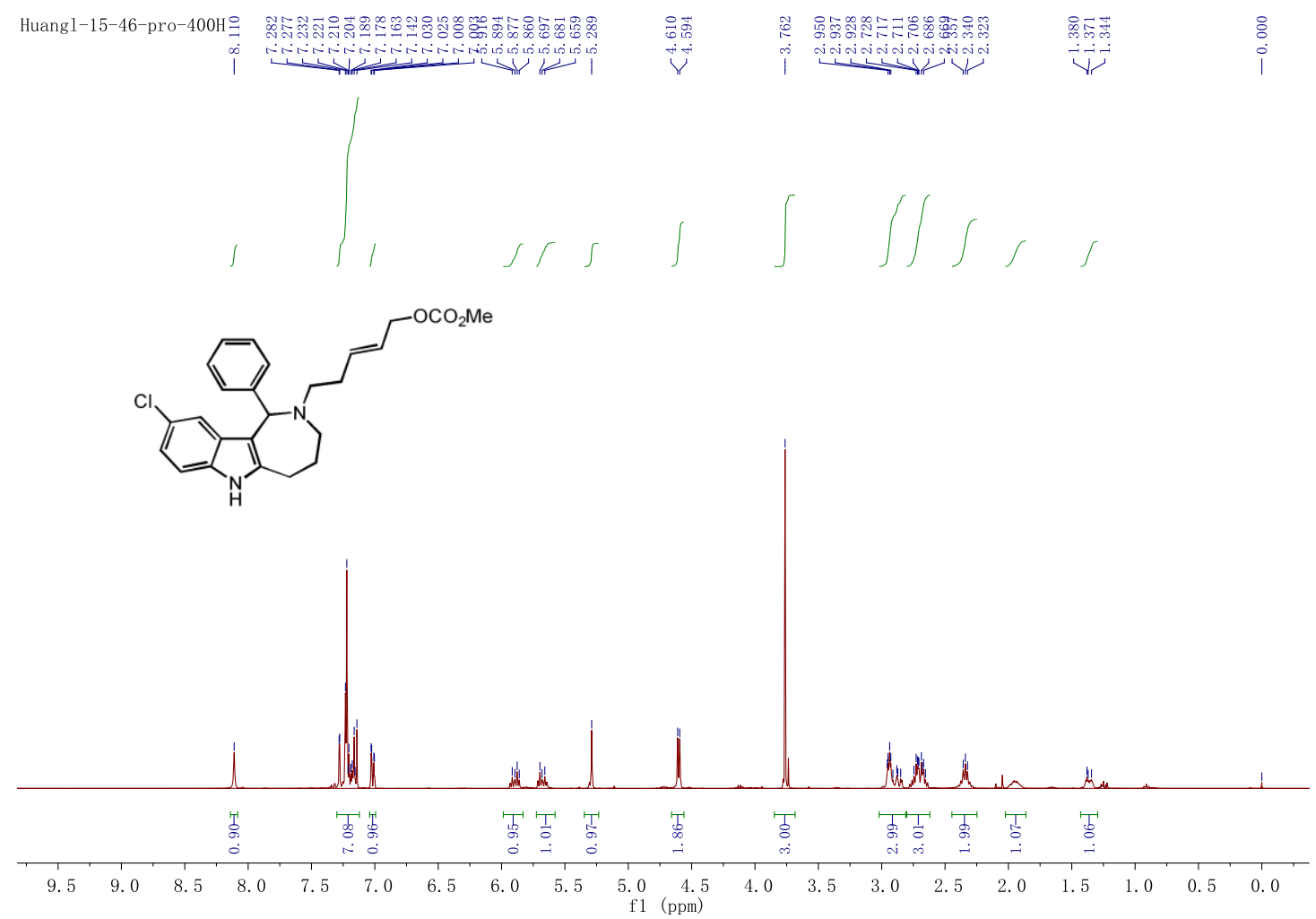

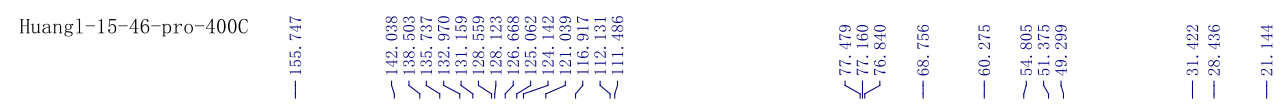
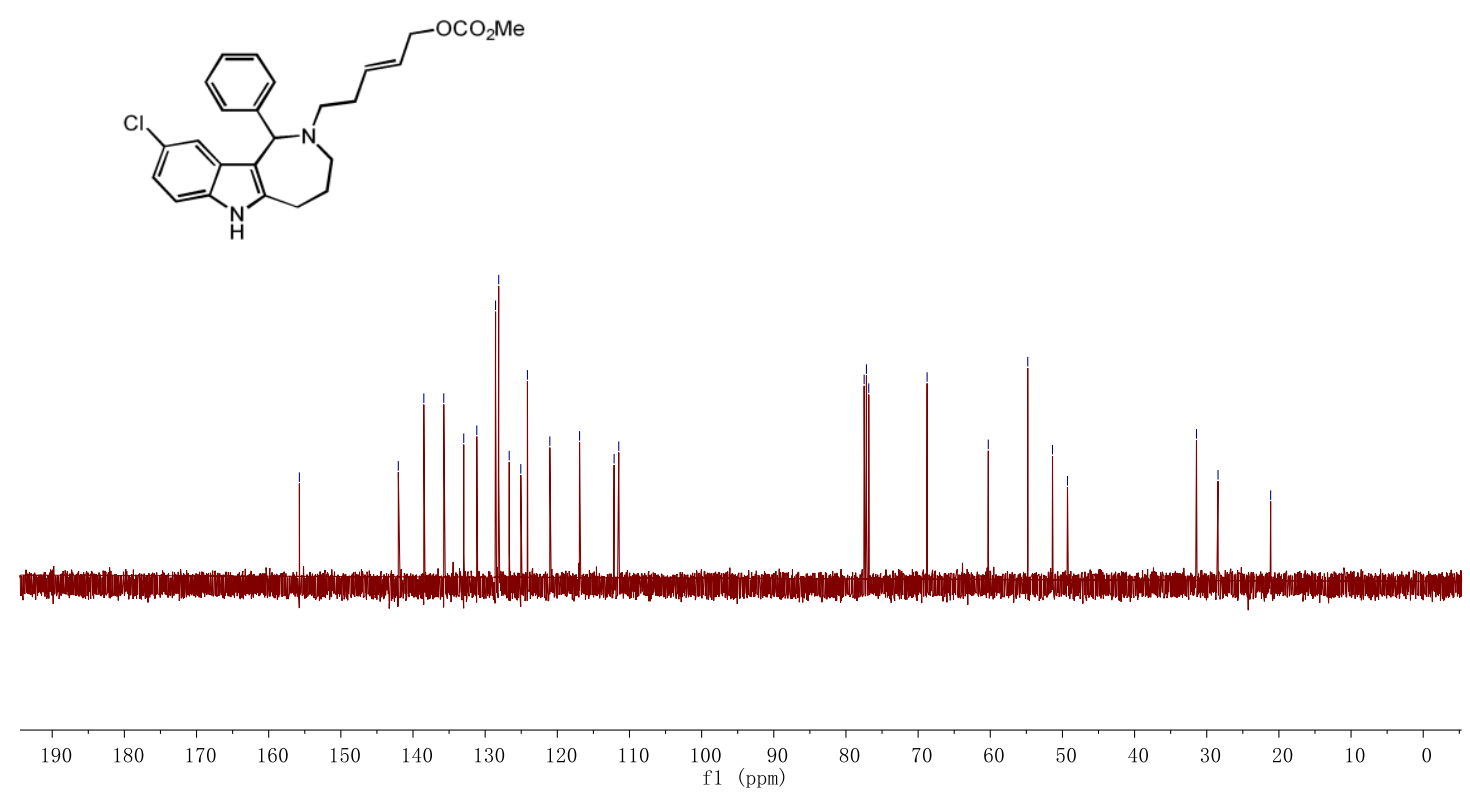

NMR Spectra of $\mathbf{1 u}$ 


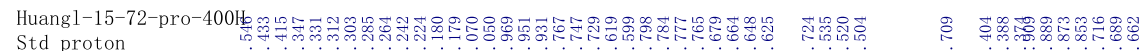
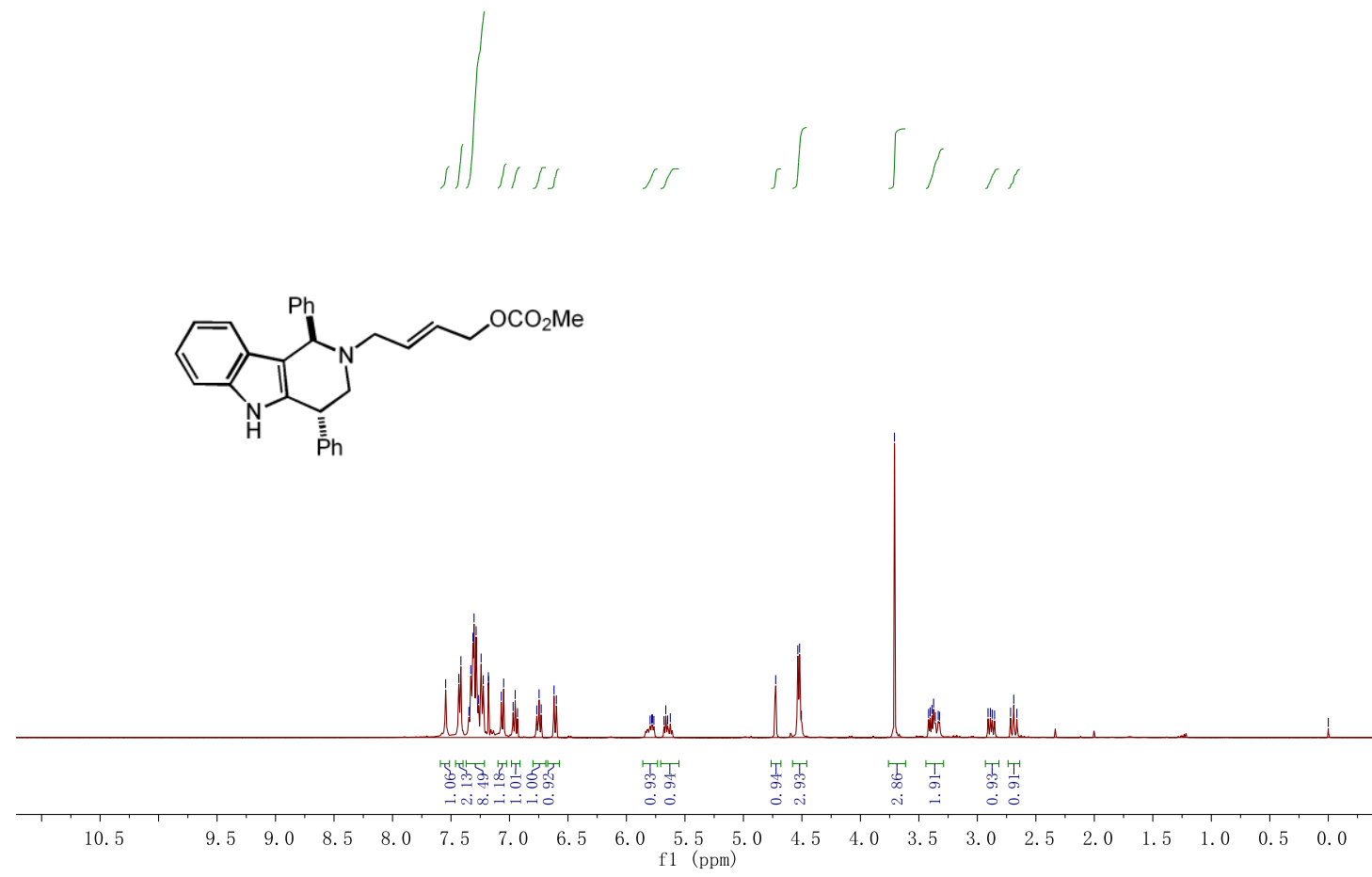

Huang1-15-72-pro-400C2 Std carbon

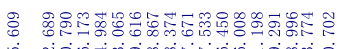

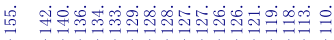
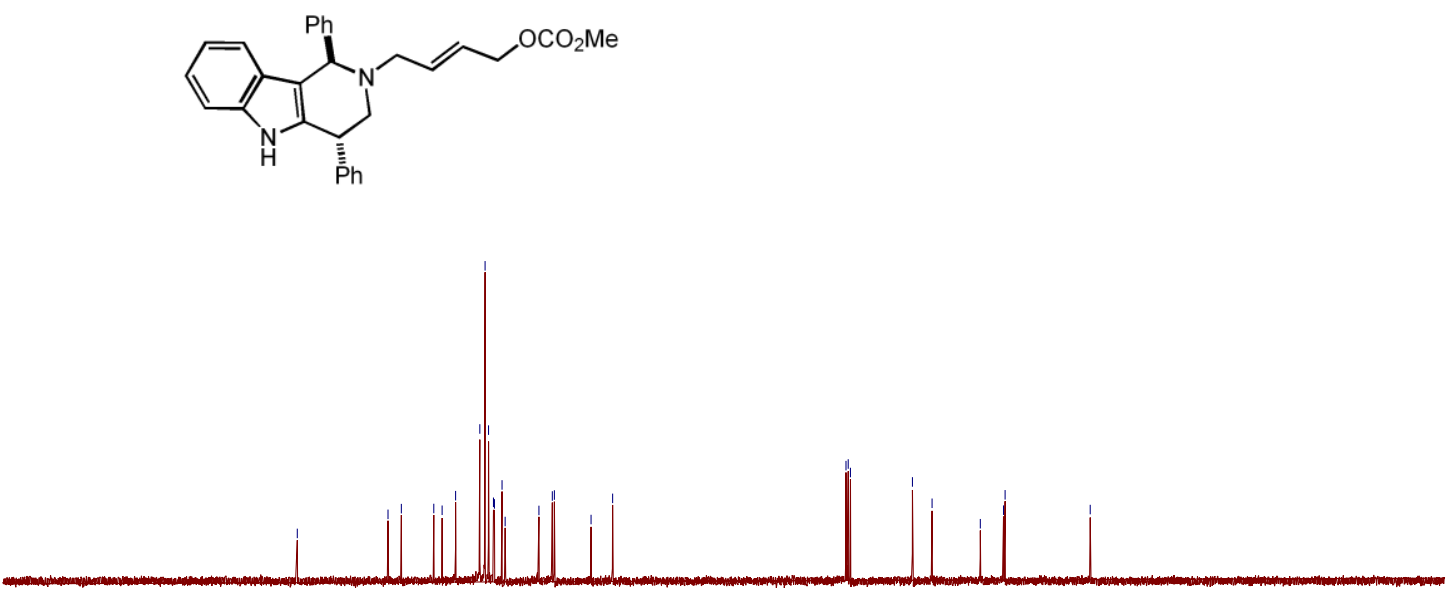

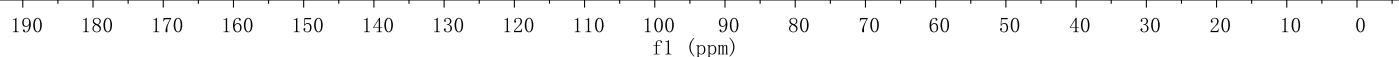

\section{Copies of 2D-NMR Spectra}



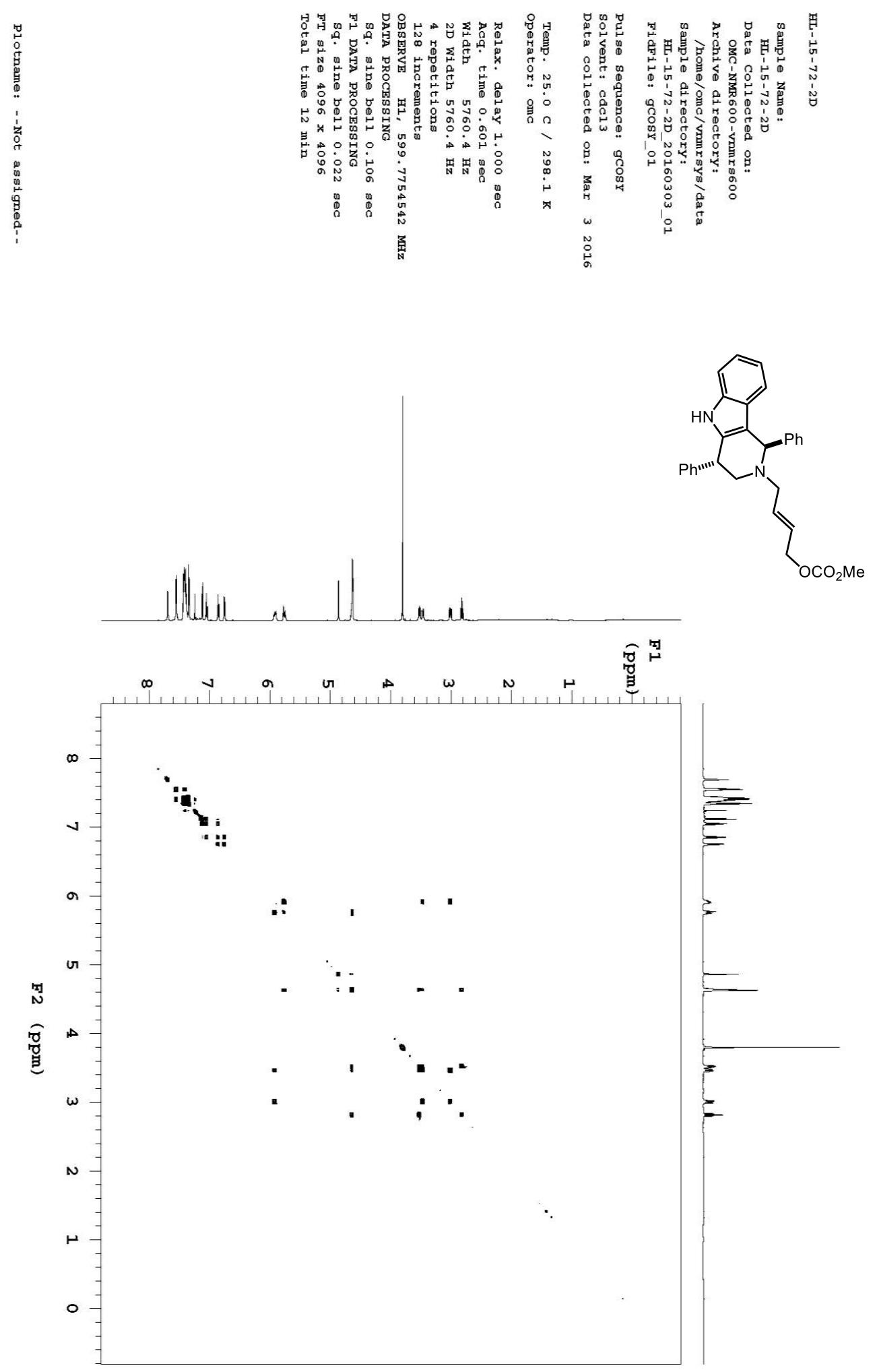

S67 


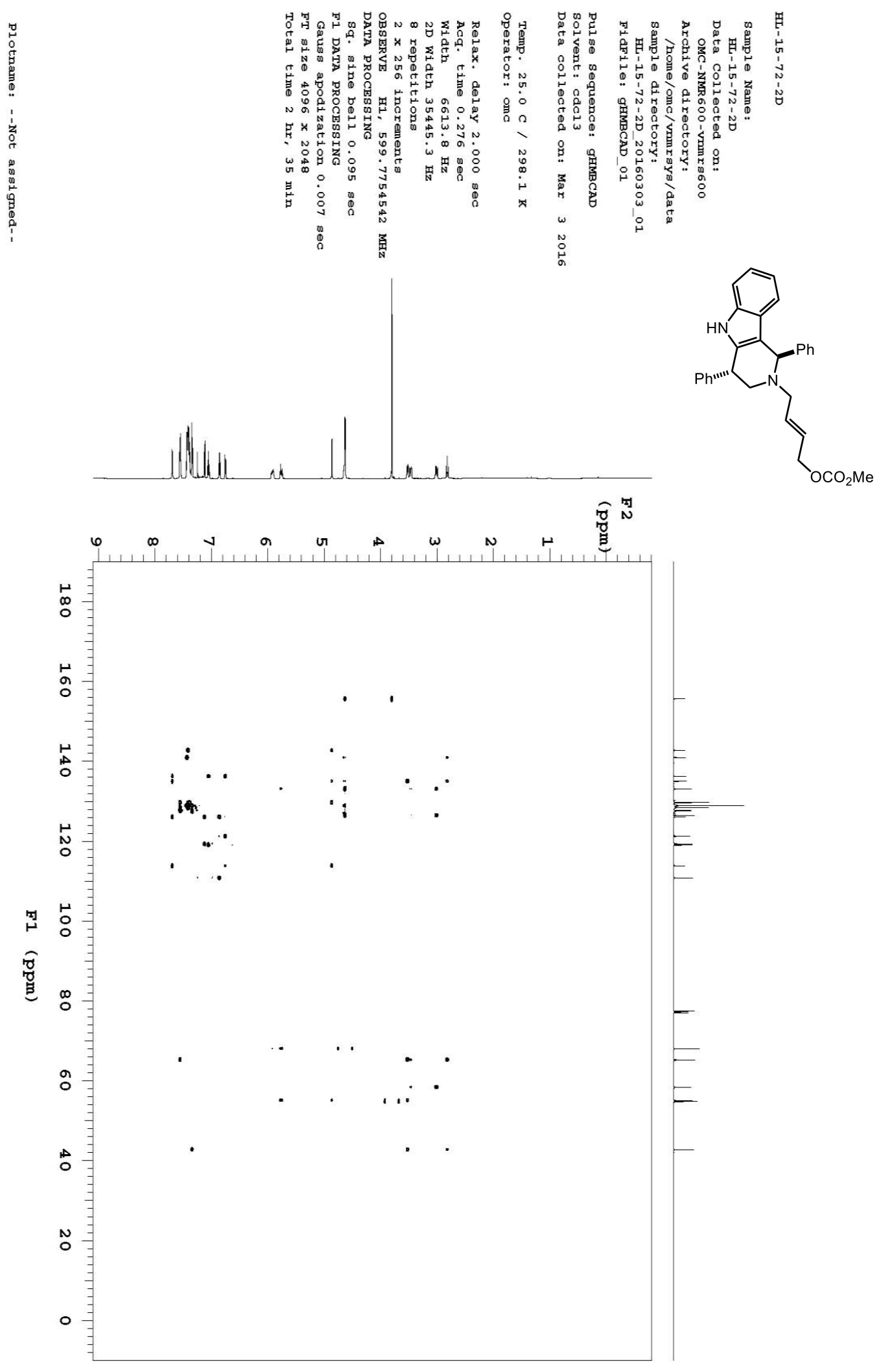



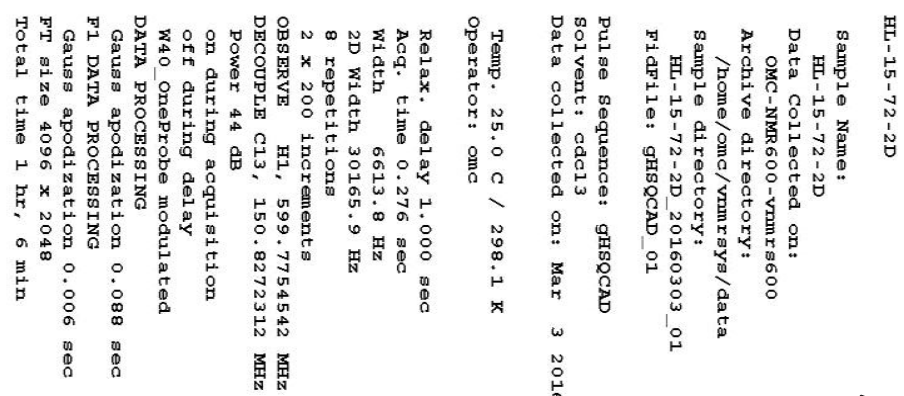

용

₹

莒

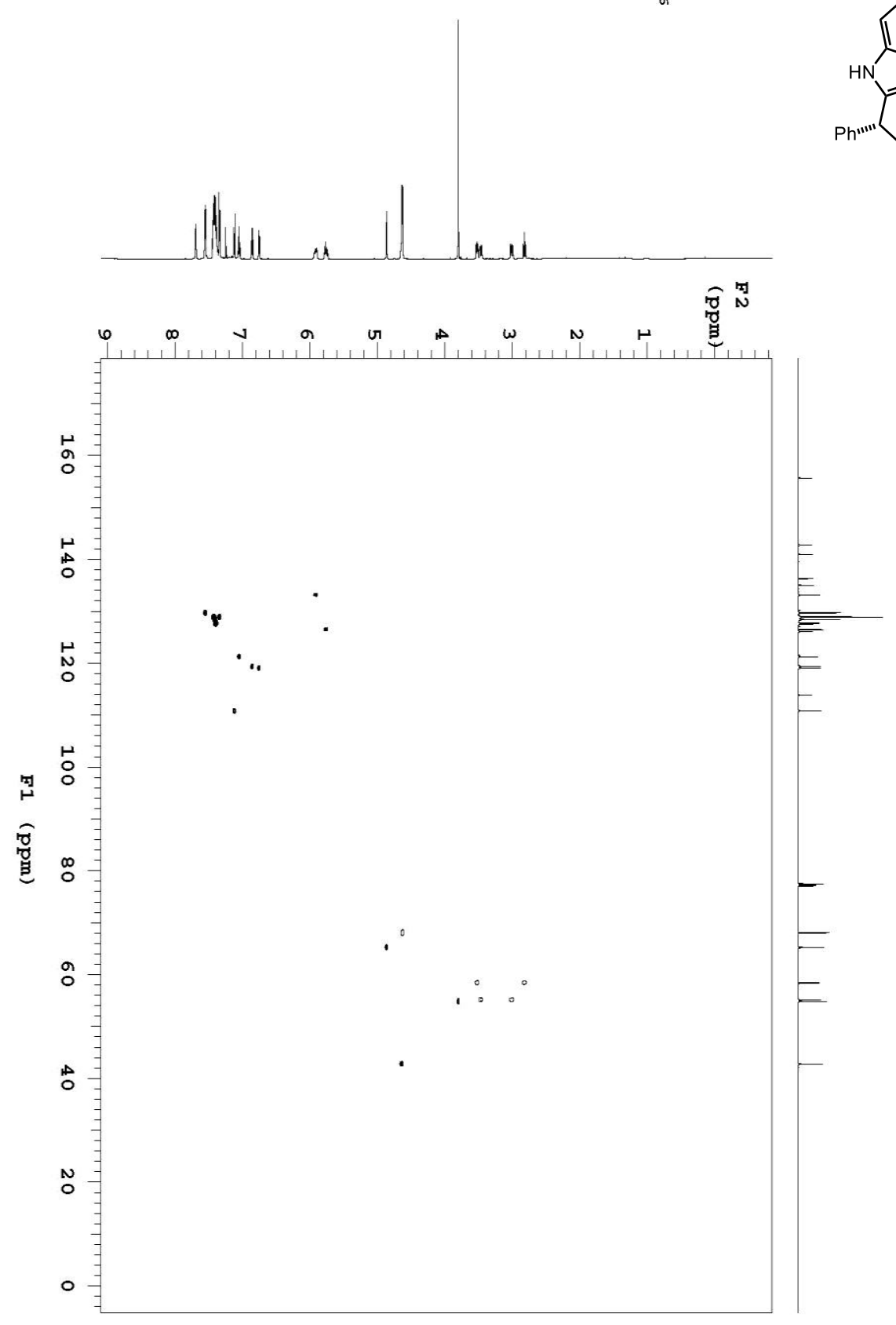



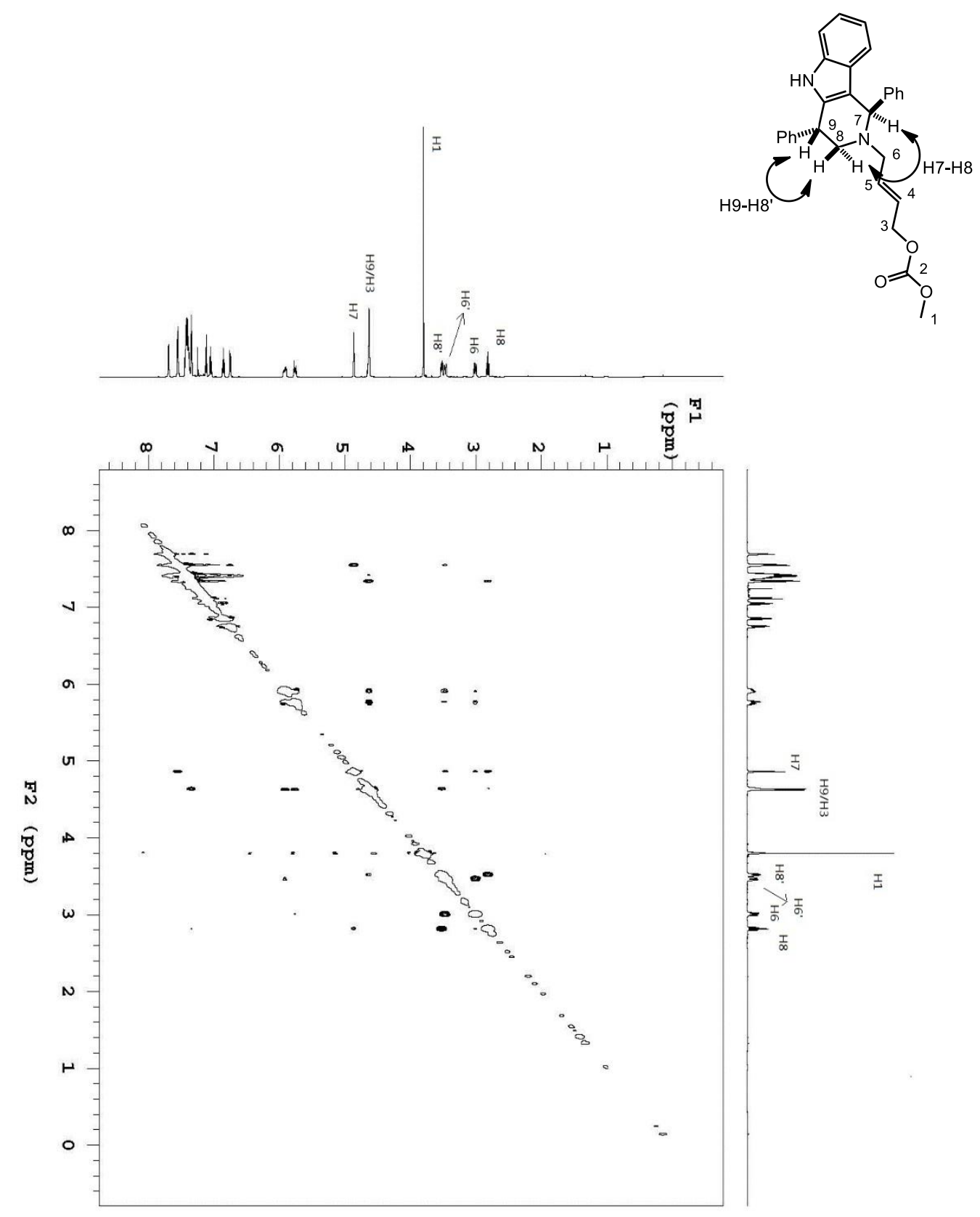


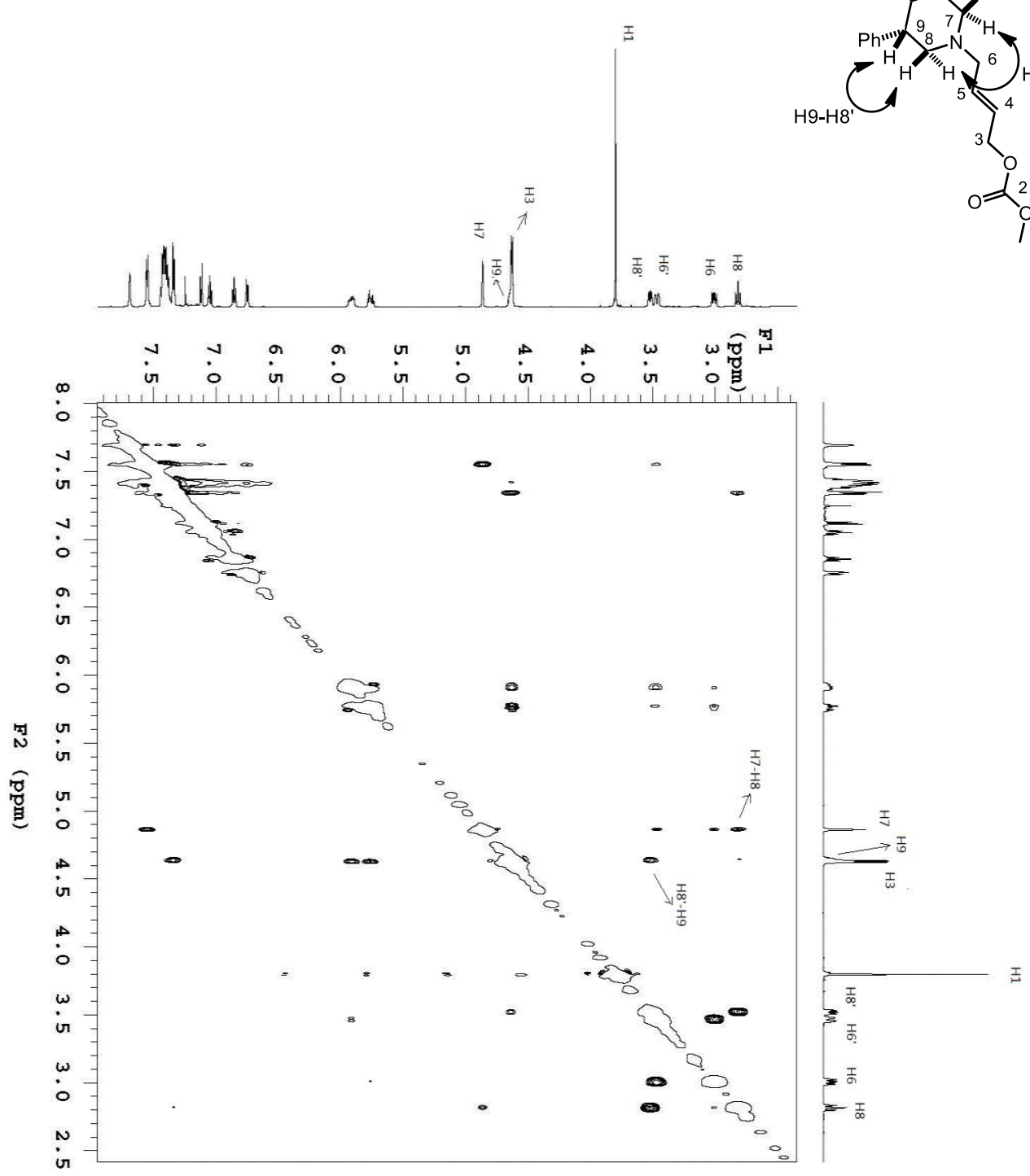




\section{NMR Spectra of $\mathbf{1 v}$}
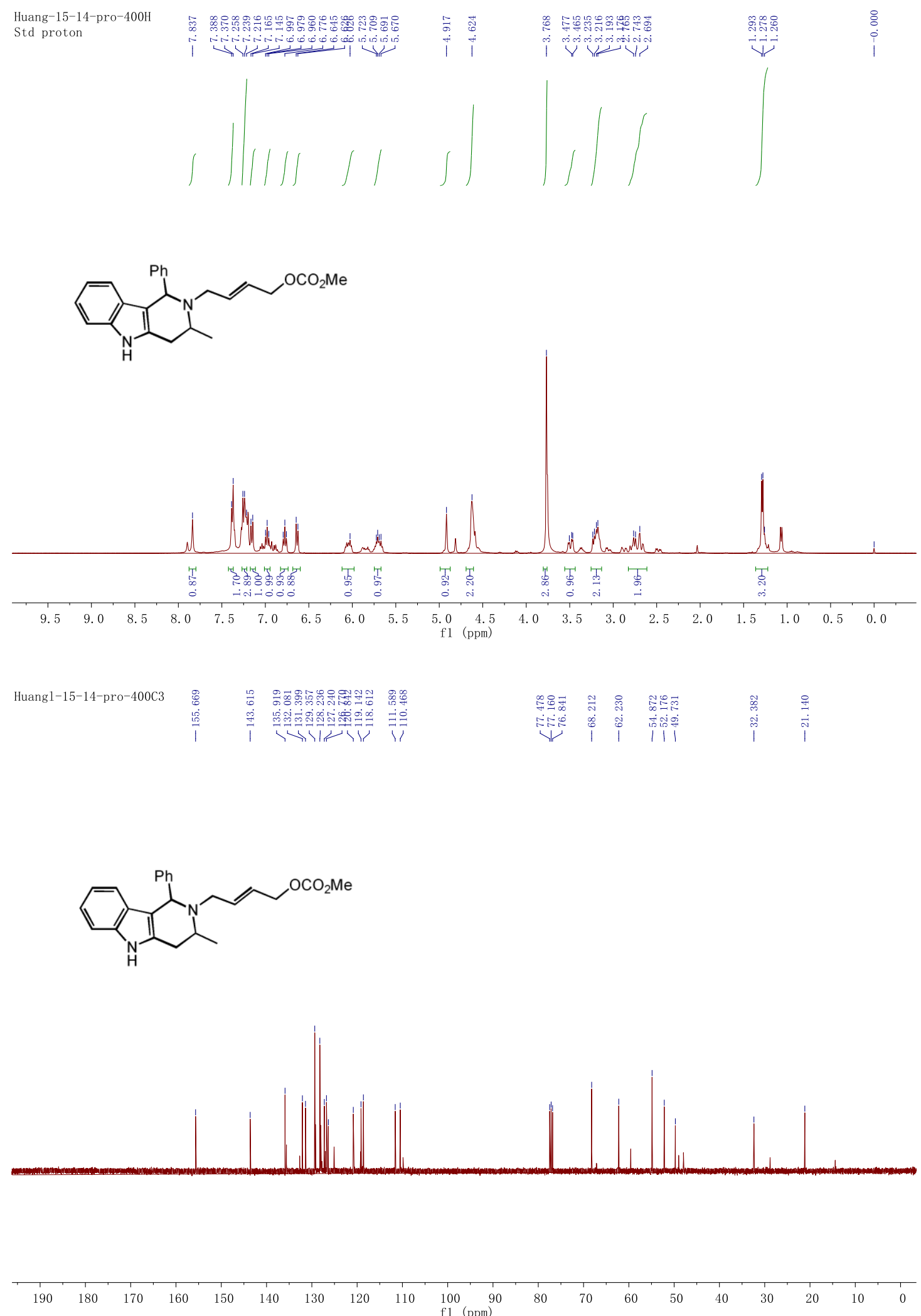
NMR Spectra of 6
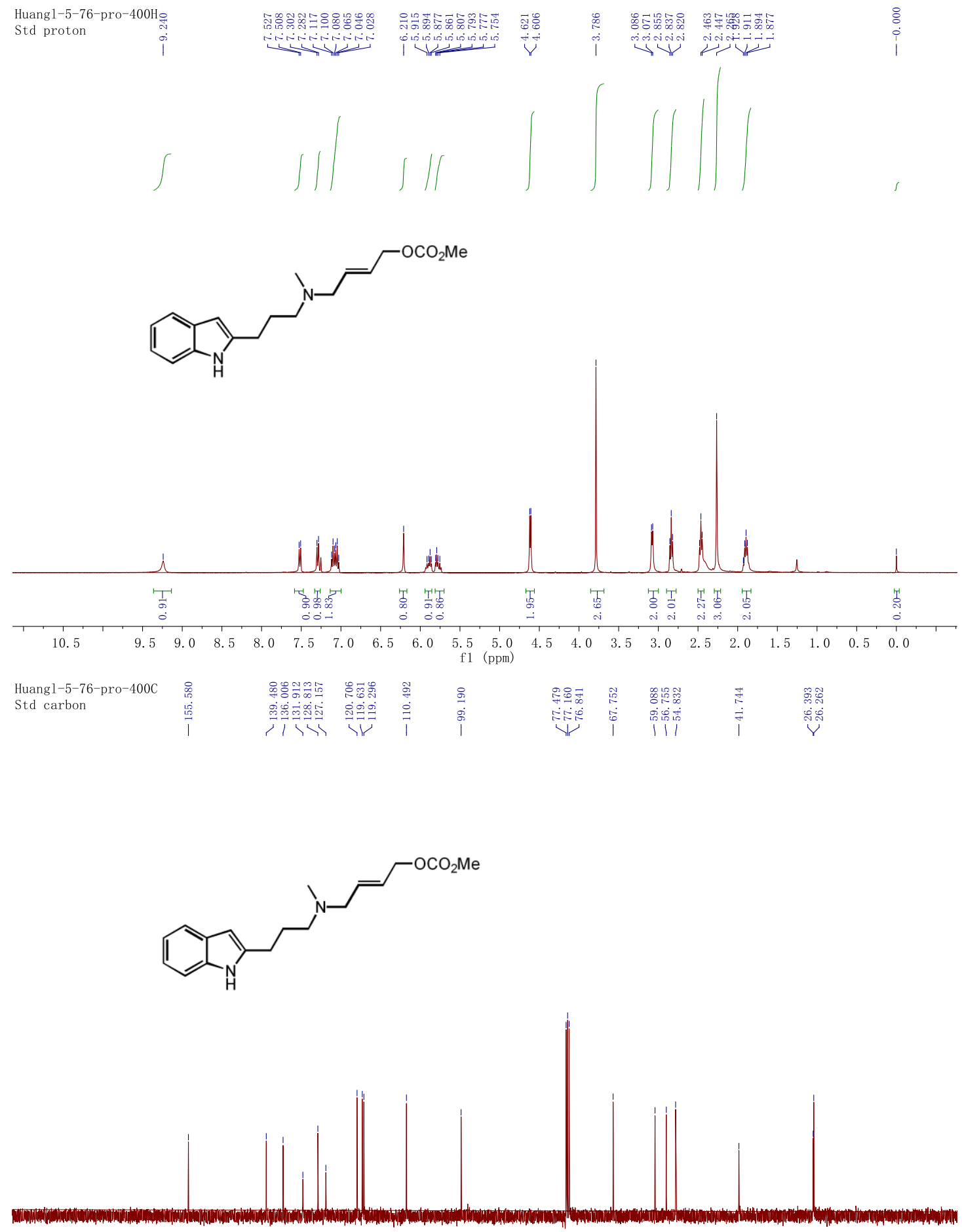

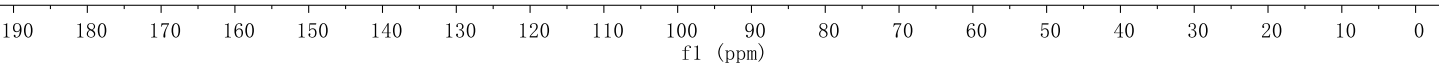


NMR Spectra and HPLC Chromatographs of 2a

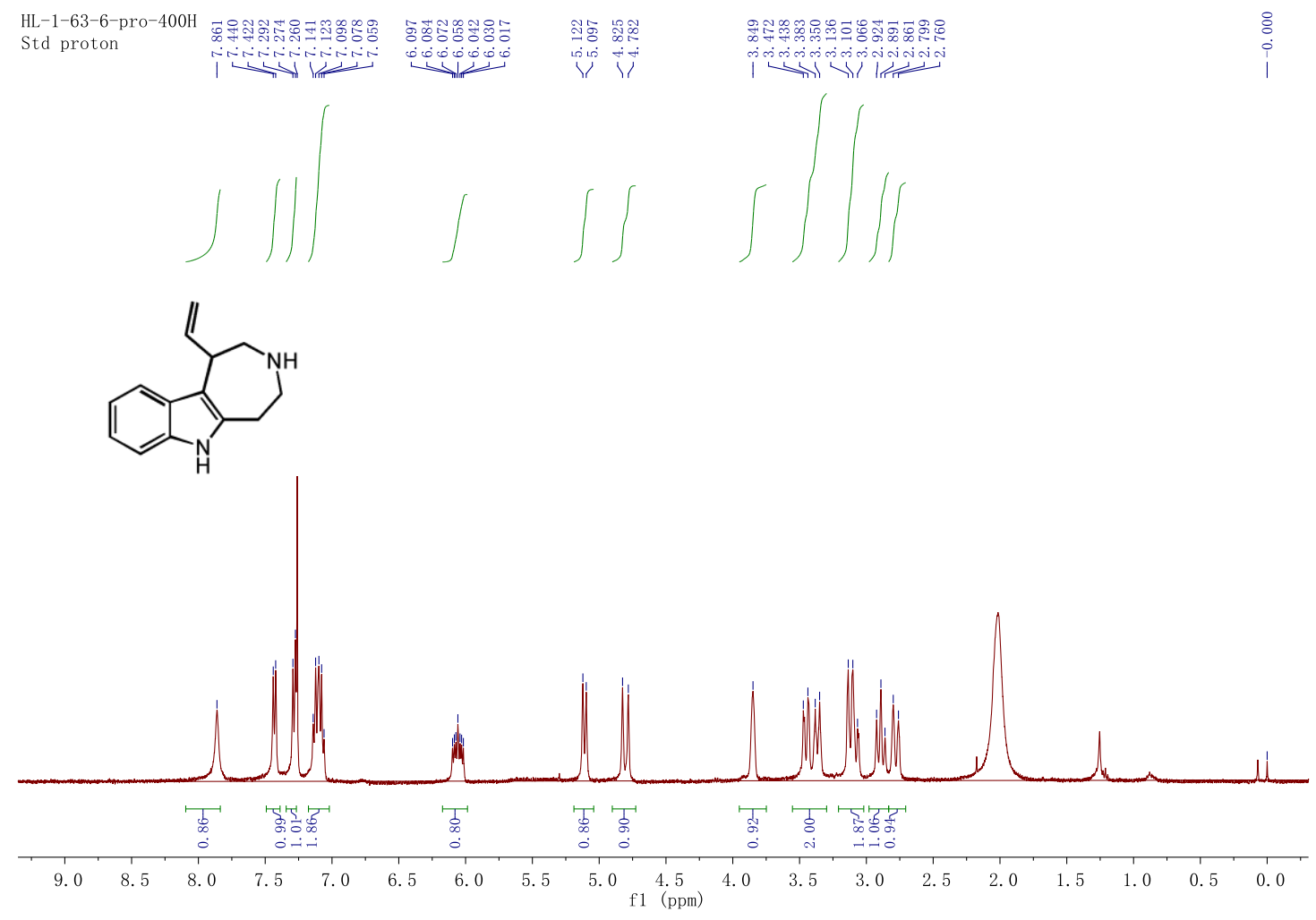

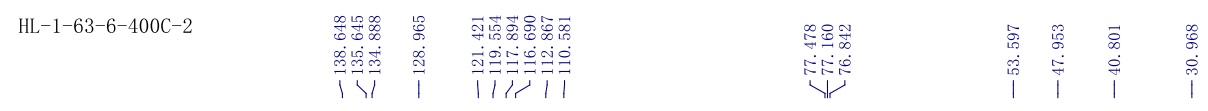<smiles>C=CC1CNCCc2[nH]c3ccccc3c21</smiles>

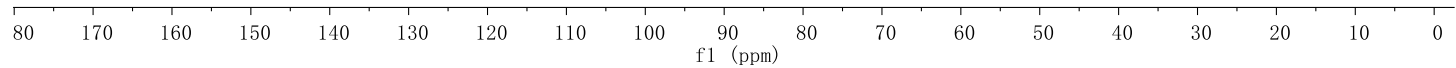




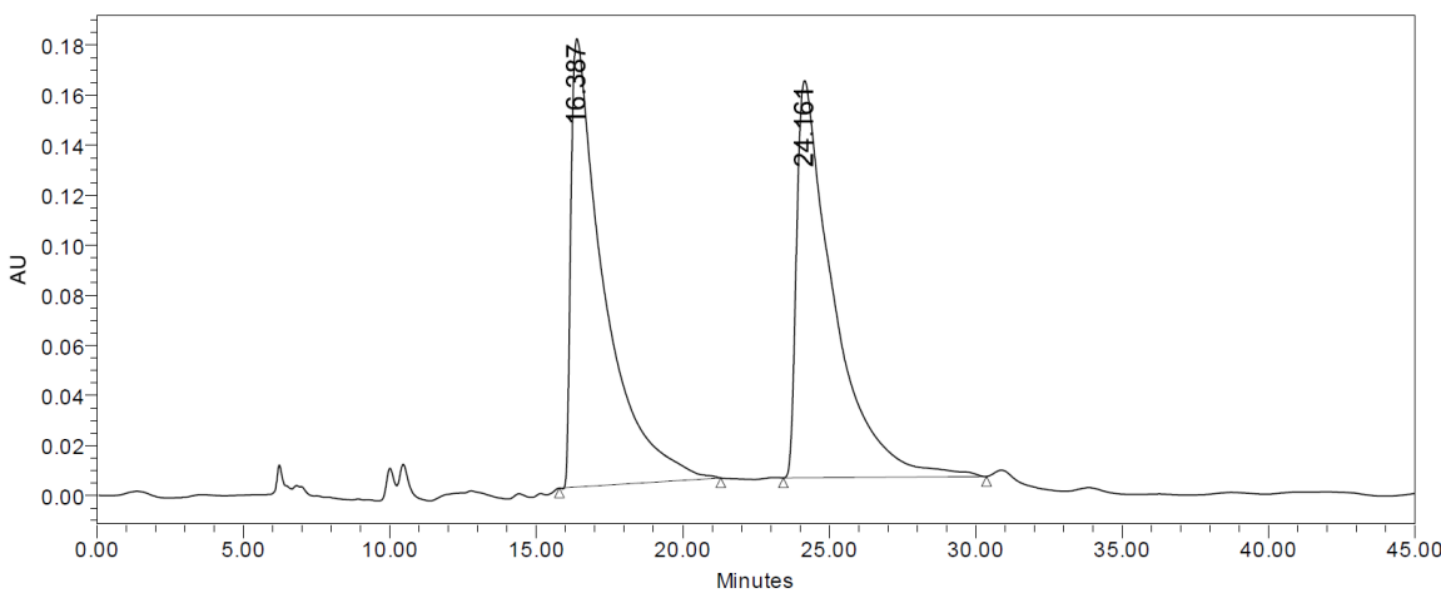

Channel: W2489 ChA; Processed Channel: W2489 ChA 230nm; Injection: 1; Date Acquired: 8/20/2014 9:19:31 PM CST; Result Id: 1354; Processing Method: a

Peak Name:

\begin{tabular}{|c|r|c|c|r|c|}
\hline & Injection & RT & Area & $\%$ Area & Height \\
\hline 1 & 1 & 24.161 & 13948691 & 49.80 & 158702 \\
\hline 2 & 1 & 16.387 & 14060027 & 50.20 & 179135 \\
\hline Mean & & 20.274 & & & \\
\hline Std. Dev. & & 5.497 & & & \\
\hline \% RSD & & 27.11 & & & \\
\hline
\end{tabular}

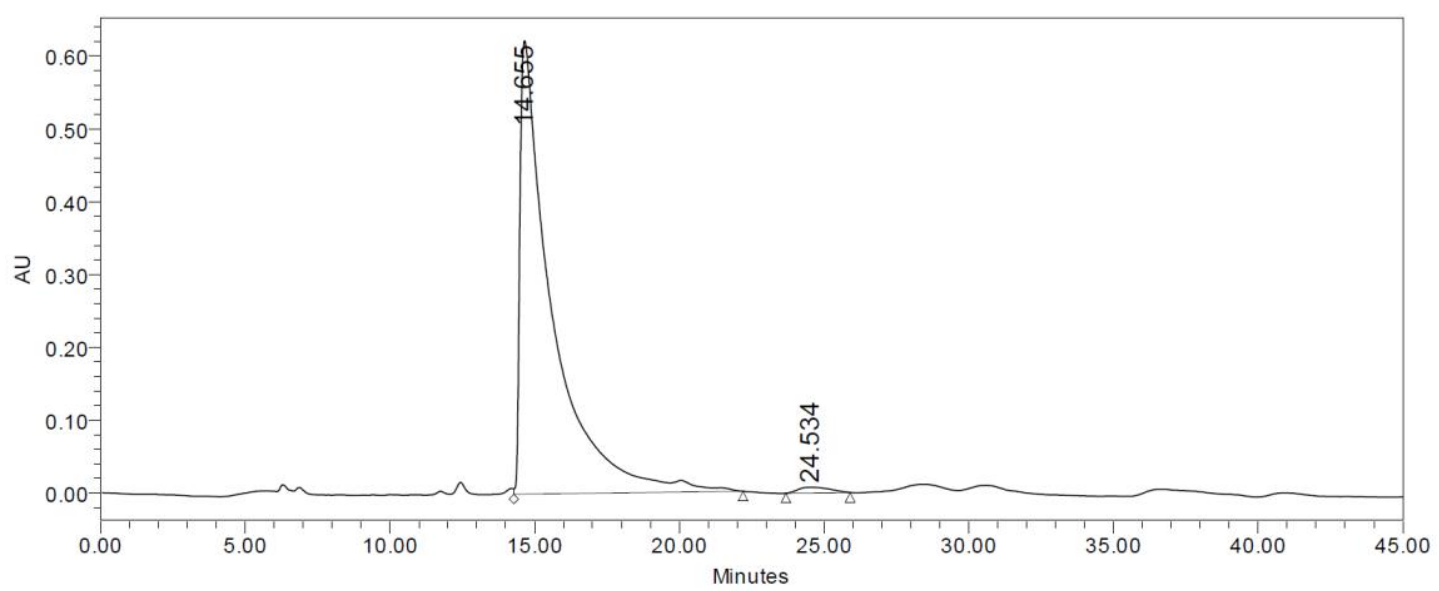

Channel: W2489 ChA; Processed Channel: W2489 ChA 230nm; Injection: 1; Date Acquired: 8/20/2014 10:04:54 PM CST; Result Id: 1511; Processing Method: qwq

Peak Name:

\begin{tabular}{|c|r|c|c|r|r|}
\hline & Injection & RT & Area & $\%$ Area & Height \\
\hline 1 & 1 & 24.534 & 595857 & 1.23 & 8058 \\
\hline 2 & 1 & 14.655 & 47850925 & 98.77 & 623102 \\
\hline Mean & & 19.595 & & & \\
\hline Std. Dev. & & 6.985 & & & \\
\hline \% RSD & & 35.65 & & & \\
\hline
\end{tabular}


NMR Spectra and HPLC Chromatographs of $\mathbf{2 b}$
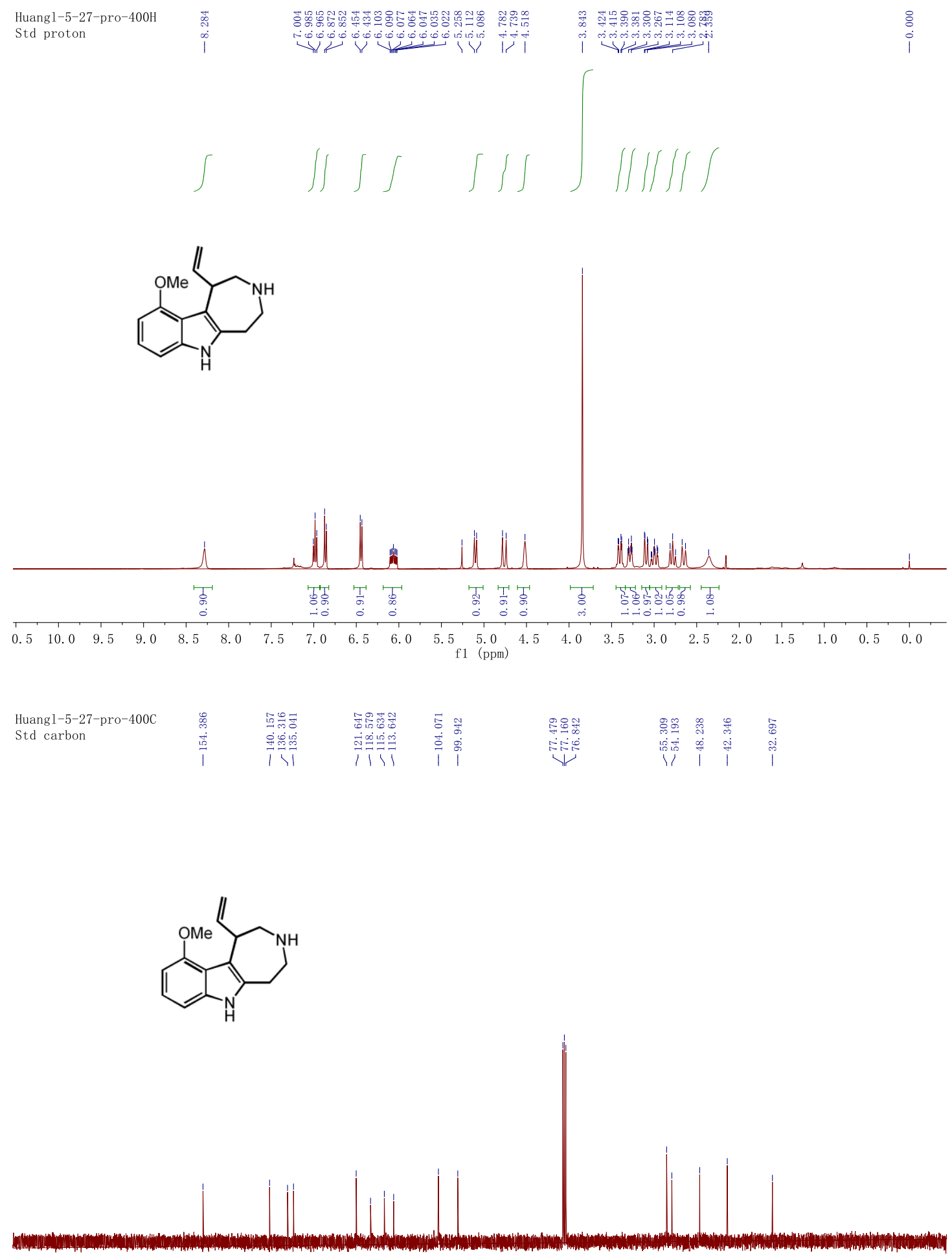

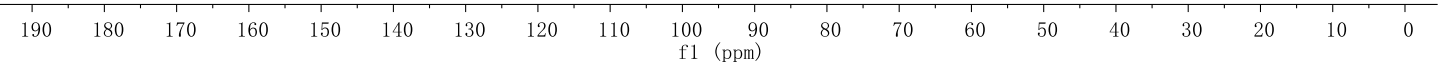




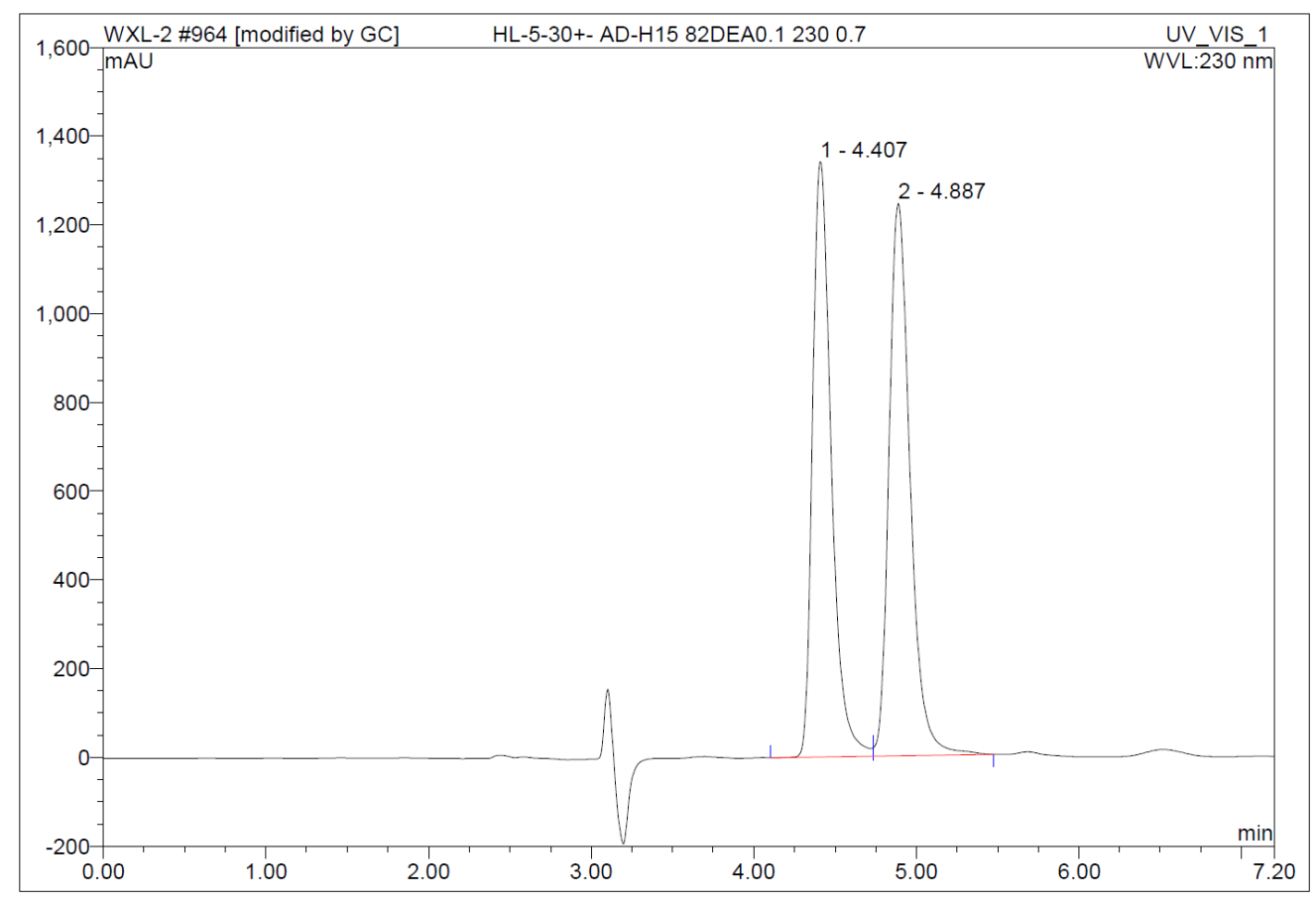

\begin{tabular}{|r|ccccccc|}
\hline No. & $\begin{array}{c}\text { Ret.Time } \\
\text { min }\end{array}$ & Peak Name & $\begin{array}{c}\text { Height } \\
\text { mAU }\end{array}$ & $\begin{array}{c}\text { Area } \\
\text { mAU*min }\end{array}$ & $\begin{array}{c}\text { Rel.Area } \\
\%\end{array}$ & Amount & Type \\
\hline 1 & 4.41 & n.a. & 1341.734 & 180.065 & 49.03 & n.a. & BM $^{*}$ \\
2 & 4.89 & n.a. & 1246.036 & 187.152 & 50.97 & n.a. & MB $^{*}$ \\
\hline Total: & & & 2587.769 & 367.217 & 100.00 & 0.000 & \\
\hline
\end{tabular}

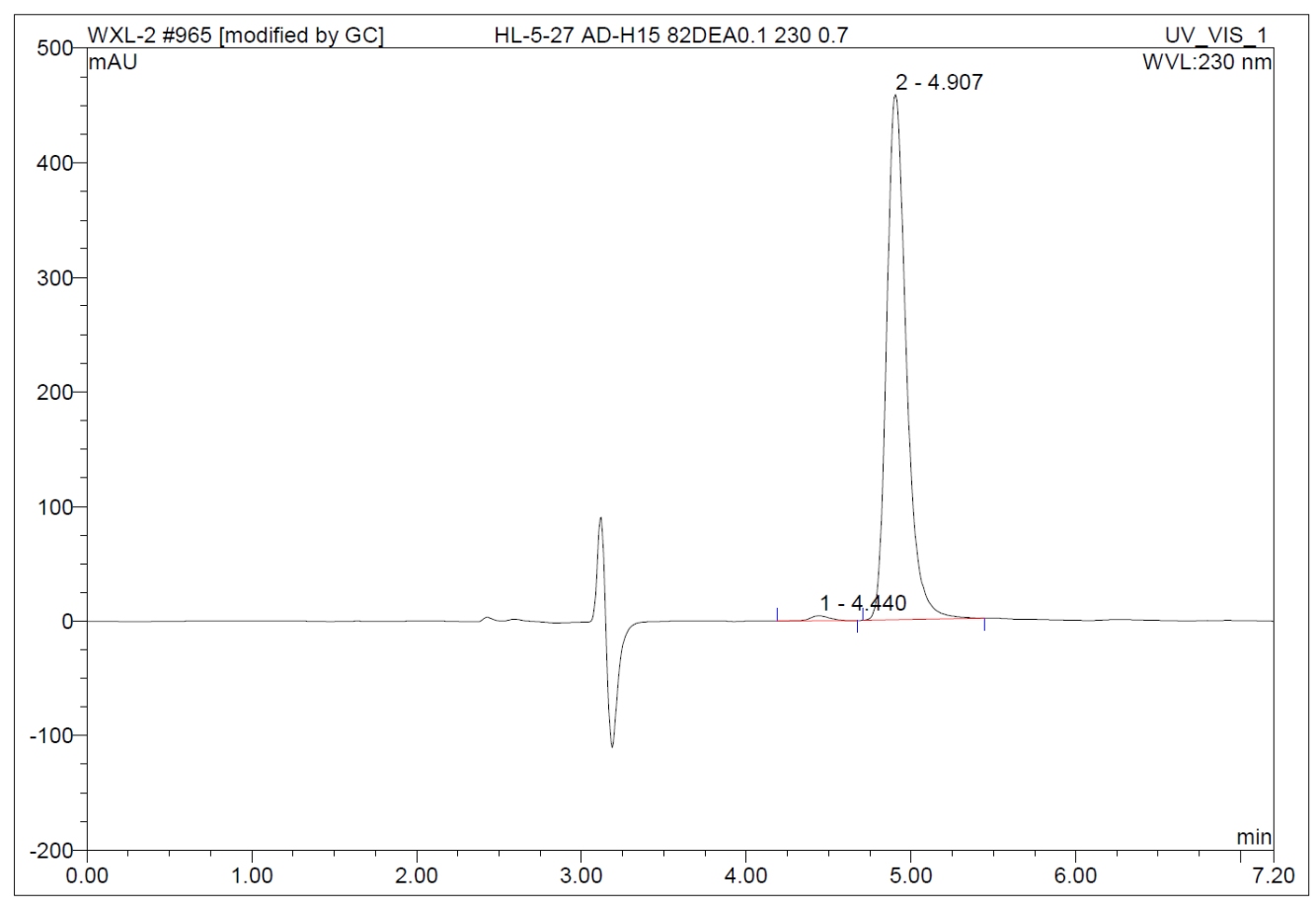

\begin{tabular}{|r|ccrrrrr|}
\hline No. & $\begin{array}{c}\text { Ret.Time } \\
\text { min }\end{array}$ & Peak Name & $\begin{array}{c}\text { Height } \\
\text { mAU }\end{array}$ & $\begin{array}{c}\text { Area } \\
\text { mAU*min }\end{array}$ & $\begin{array}{r}\text { Rel.Area } \\
\%\end{array}$ & Amount & Type \\
\hline 1 & 4.44 & n.a. & 4.328 & 0.581 & 0.91 & n.a. & BMB $^{*}$ \\
2 & 4.91 & n.a. & 458.431 & 63.354 & 99.09 & n.a. & BMB $^{*}$ \\
\hline Total: & & & 462.759 & 63.935 & 100.00 & 0.000 & \\
\hline
\end{tabular}


NMR Spectra and HPLC Chromatographs of 2c

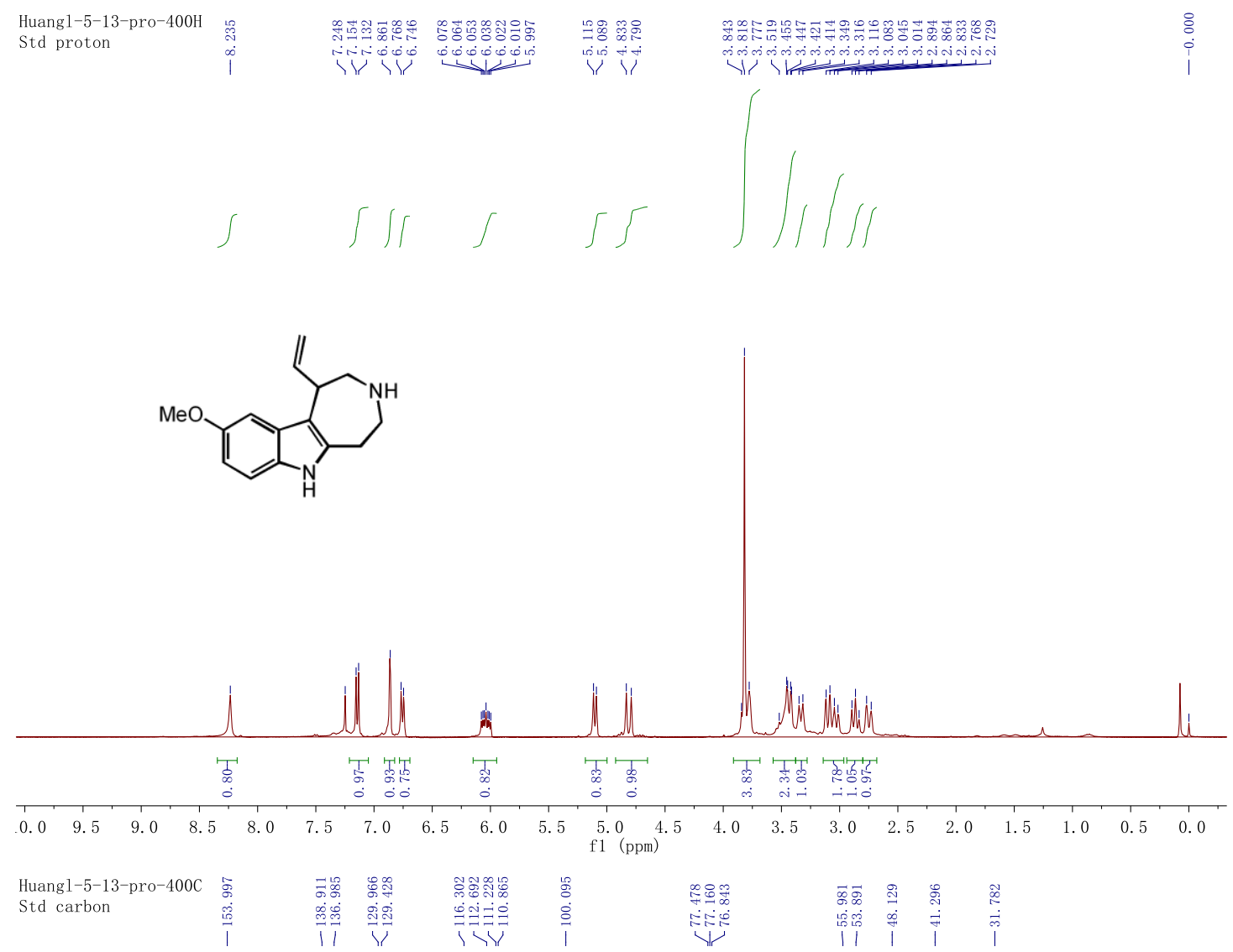<smiles>C=CC1CNCCc2[nH]c3ccc(OC)cc3c21</smiles>

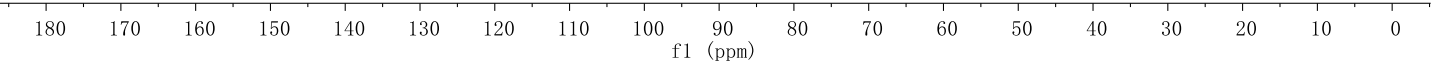




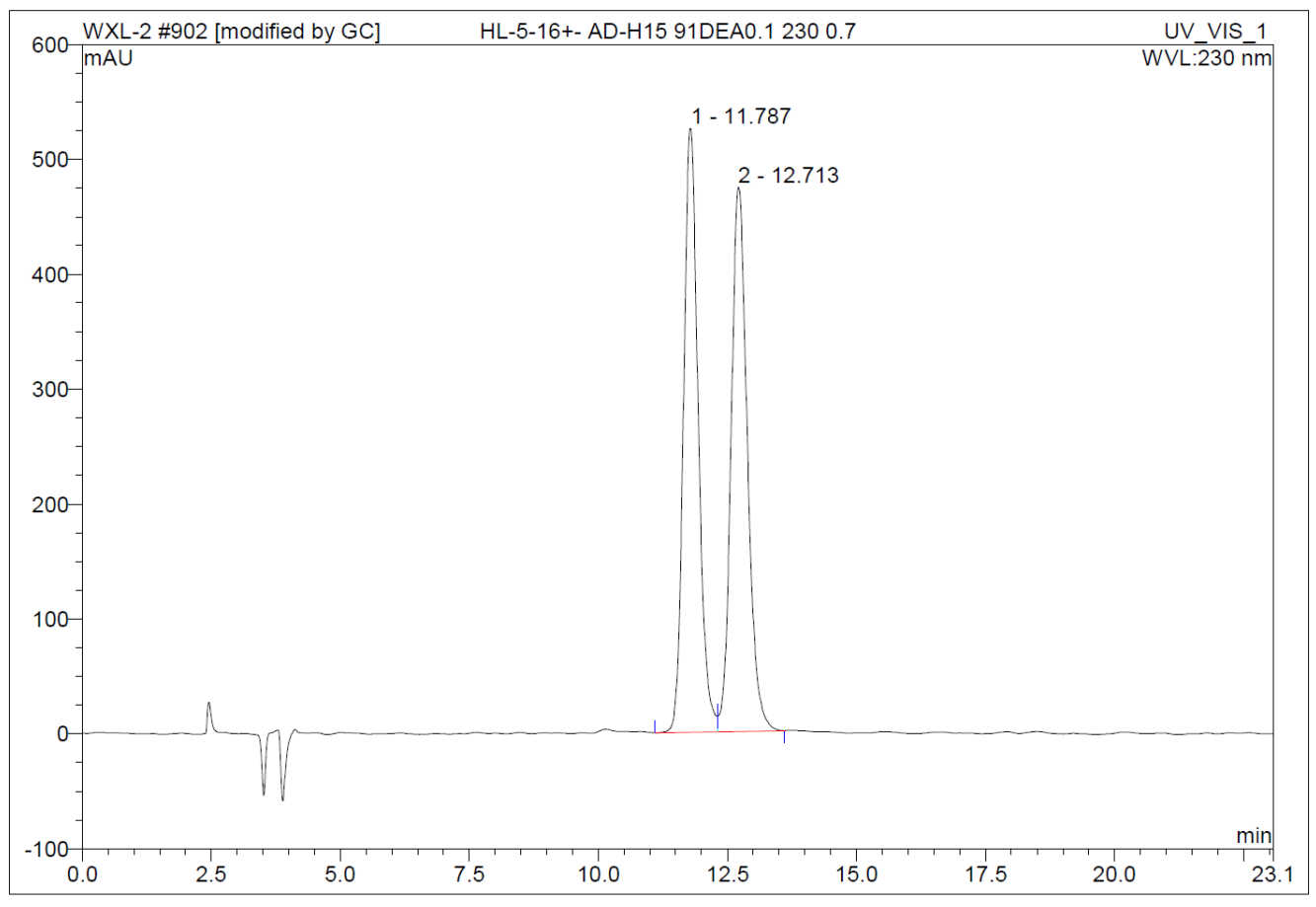

\begin{tabular}{|r|ccccccc|}
\hline No. & $\begin{array}{c}\text { Ret.Time } \\
\text { min }\end{array}$ & Peak Name & $\begin{array}{c}\text { Height } \\
\text { mAU }\end{array}$ & $\begin{array}{c}\text { Area } \\
\text { mAU*min }\end{array}$ & $\begin{array}{r}\text { Rel.Area } \\
\%\end{array}$ & Amount & Type \\
\hline 1 & 11.79 & n.a. & 525.426 & 172.775 & 49.93 & n.a. & BM $^{*}$ \\
2 & 12.71 & n.a. & 473.840 & 173.291 & 50.07 & n.a. & MB $^{*}$ \\
\hline Total: & & & 999.267 & 346.065 & 100.00 & 0.000 & \\
\hline
\end{tabular}

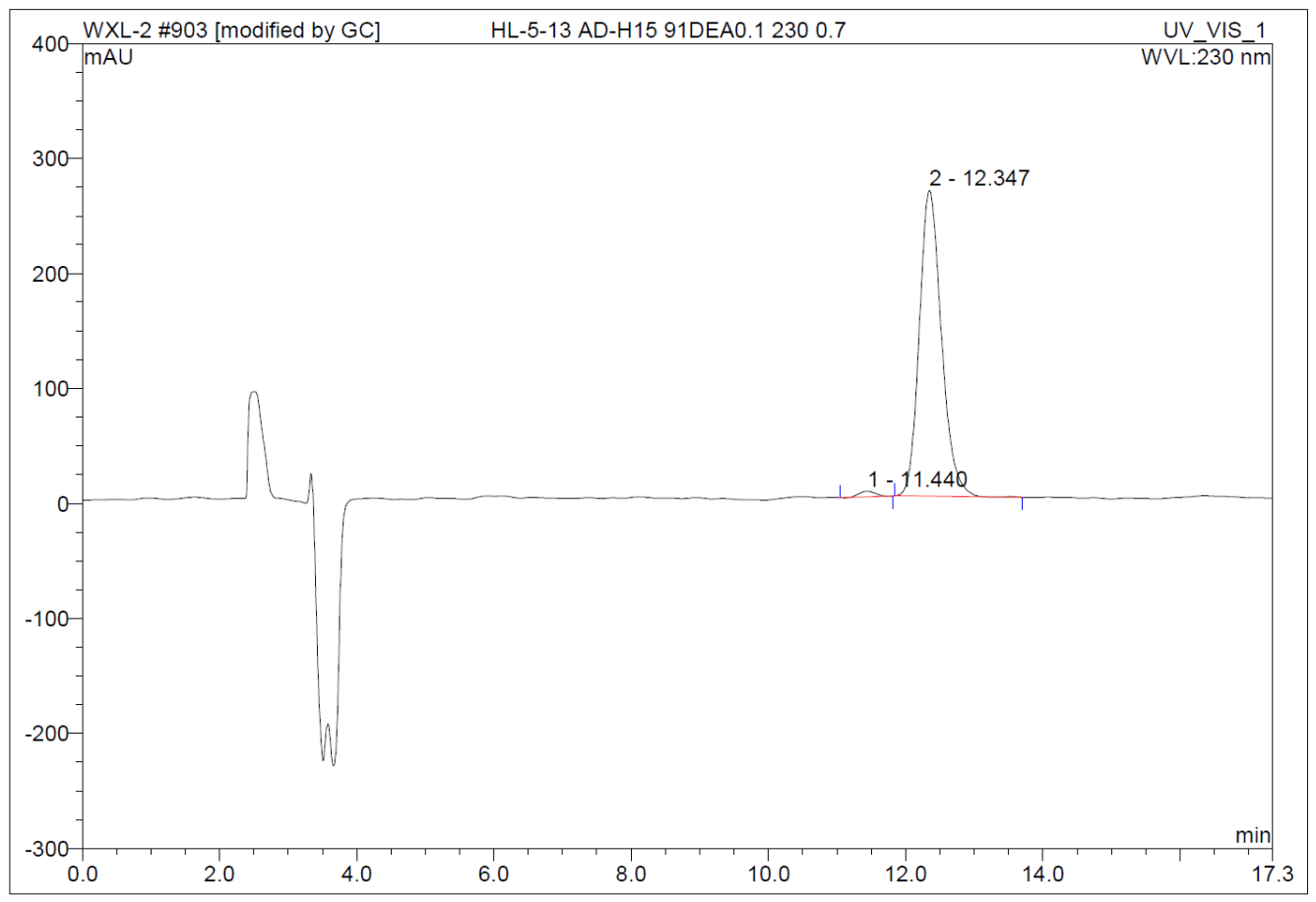

\begin{tabular}{|r|ccrrrrr|}
\hline No. & $\begin{array}{c}\text { Ret.Time } \\
\text { min }\end{array}$ & Peak Name & $\begin{array}{c}\text { Height } \\
\text { mAU }\end{array}$ & $\begin{array}{c}\text { Area } \\
\text { mAU*min }\end{array}$ & $\begin{array}{r}\text { Rel.Area } \\
\%\end{array}$ & Amount & Type \\
\hline 1 & 11.44 & n.a. & 4.805 & 1.292 & 1.28 & n.a. & BMB $^{*}$ \\
2 & 12.35 & n.a. & 265.926 & 99.337 & 98.72 & n.a. & BMB $^{*}$ \\
\hline Total: & & & 270.731 & 100.629 & 100.00 & 0.000 & \\
\hline
\end{tabular}


NMR Spectra and HPLC Chromatographs of 2d

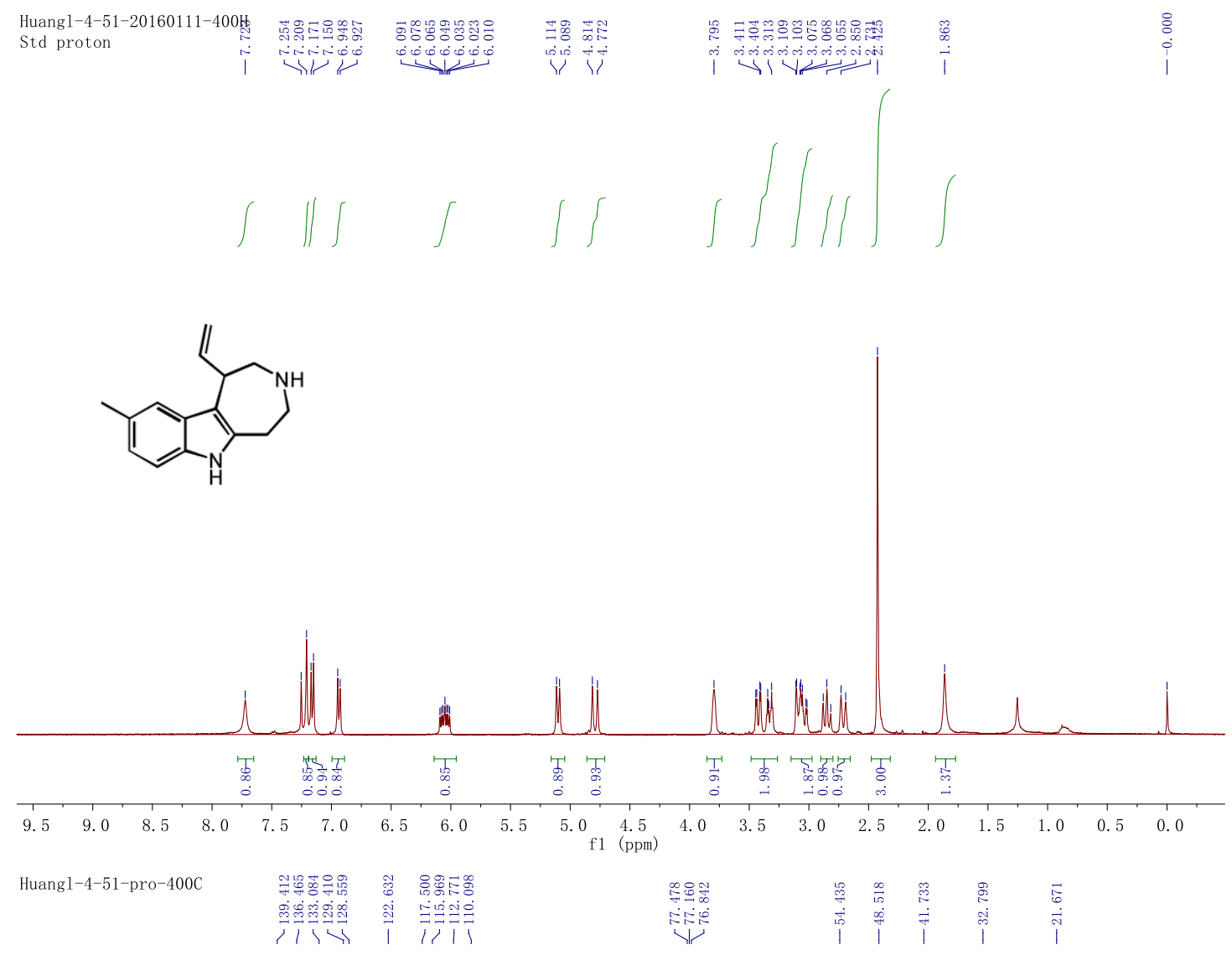<smiles>C=CC1CNCCc2[nH]c3ccc(C)cc3c21</smiles>

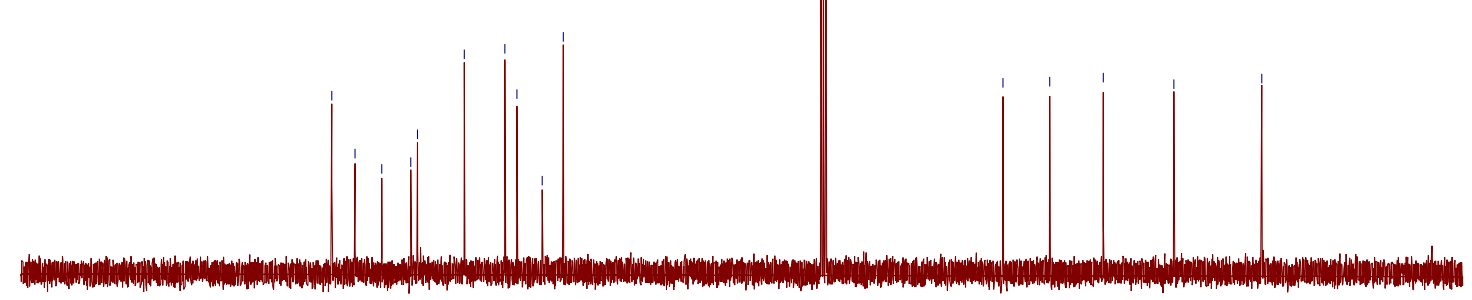

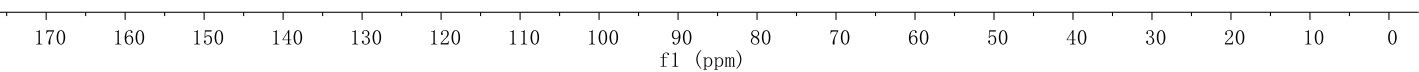




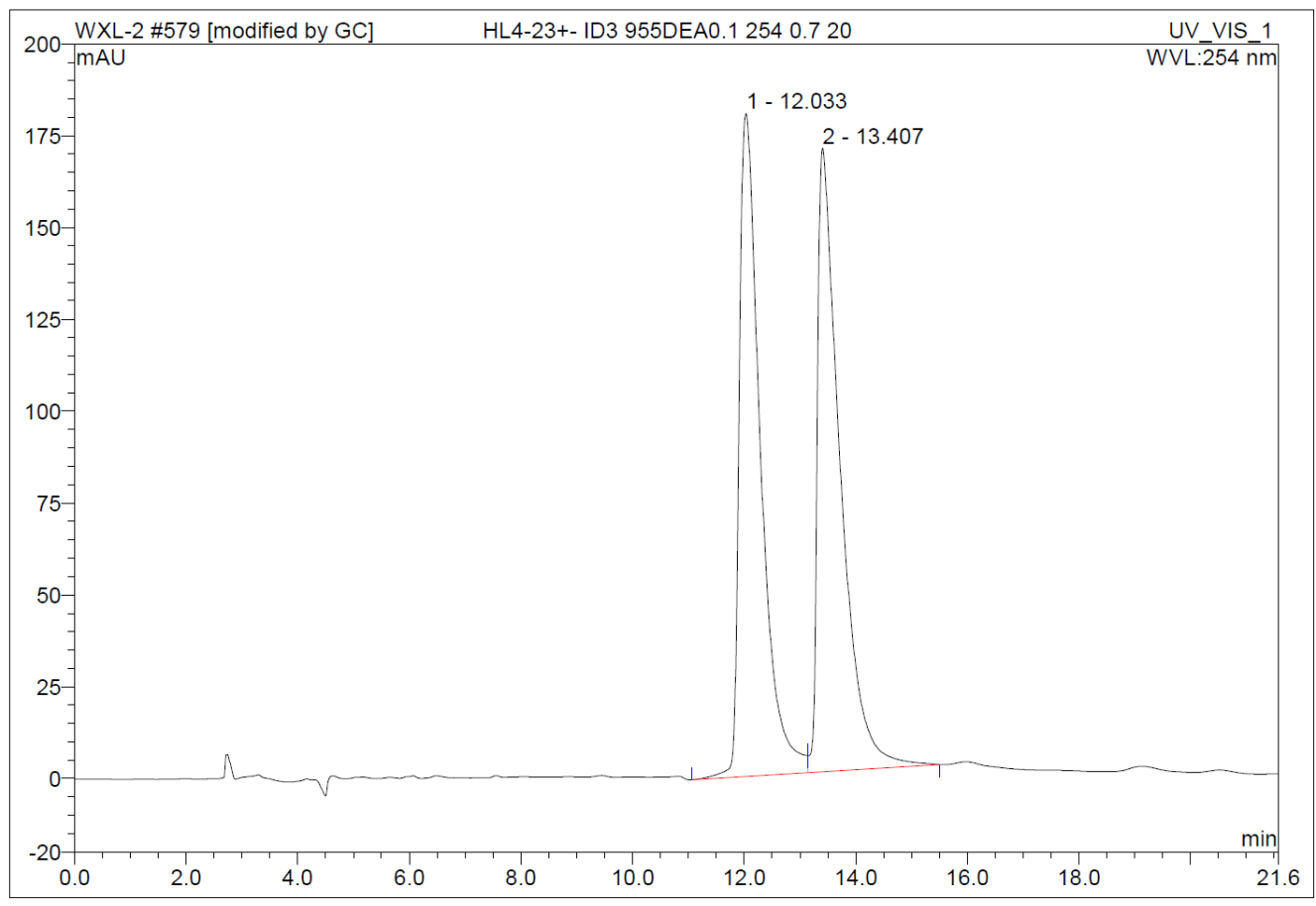

\begin{tabular}{|r|ccrrrrr|}
\hline No. & $\begin{array}{c}\text { Ret.Time } \\
\text { min }\end{array}$ & Peak Name & $\begin{array}{c}\text { Height } \\
\text { mAU }\end{array}$ & $\begin{array}{r}\text { Area } \\
\text { mAU*min }\end{array}$ & $\begin{array}{r}\text { Rel.Area } \\
\%\end{array}$ & Amount & Type \\
\hline 1 & 12.03 & n.a. & 180.506 & 80.175 & 49.86 & n.a. & BM \\
2 & 13.41 & n.a. & 169.789 & 80.632 & 50.14 & n.a. & MB $^{*}$ \\
\hline Total: & & & 350.296 & 160.807 & 100.00 & 0.000 & \\
\hline
\end{tabular}

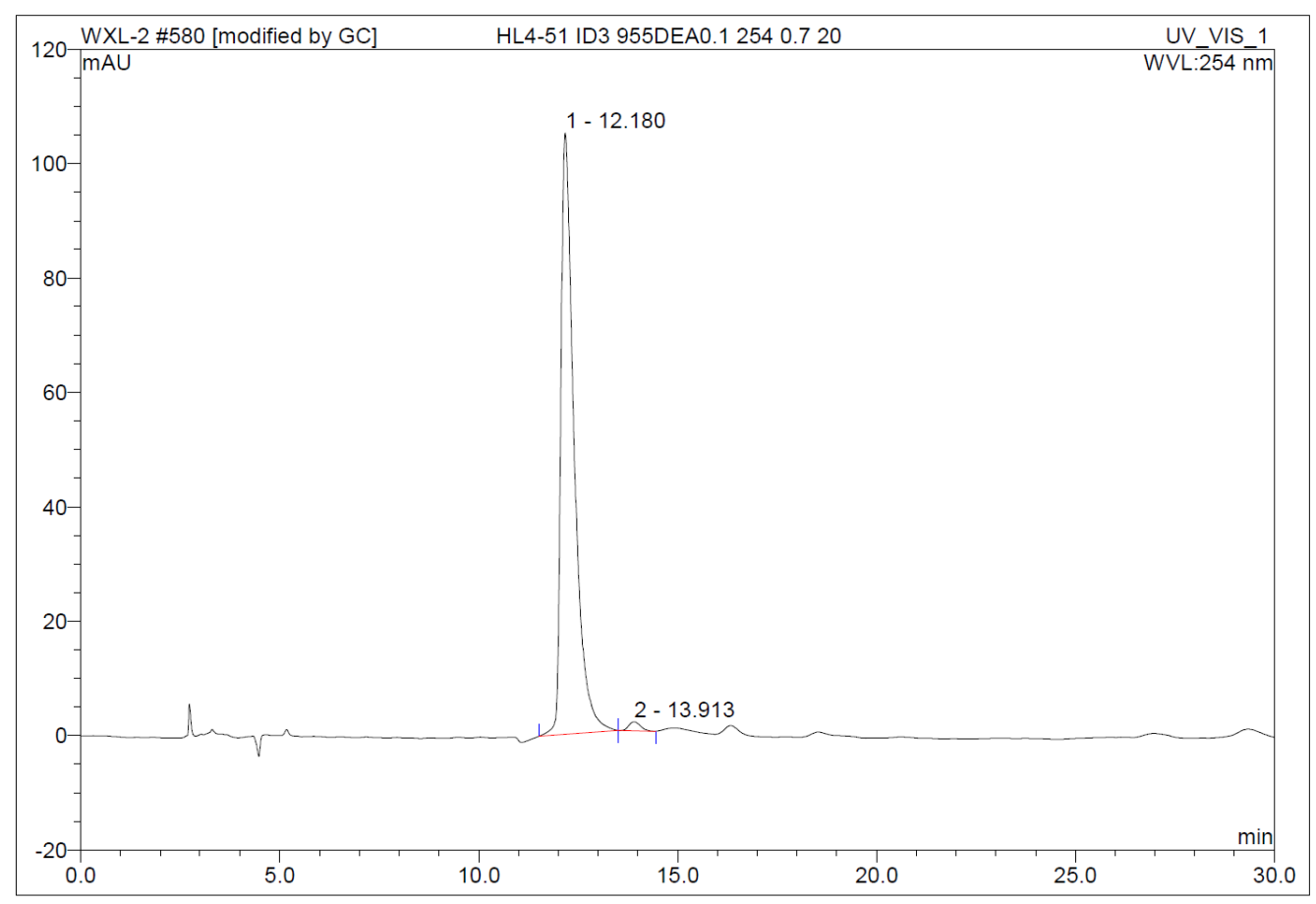

\begin{tabular}{|r|ccrrrrr|}
\hline No. & $\begin{array}{c}\text { Ret.Time } \\
\text { min }\end{array}$ & Peak Name & $\begin{array}{c}\text { Height } \\
\text { mAU }\end{array}$ & $\begin{array}{r}\text { Area } \\
\text { mAU*min }\end{array}$ & $\begin{array}{r}\text { Rel.Area } \\
\%\end{array}$ & Amount & Type \\
\hline 1 & 12.18 & n.a. & 105.188 & 40.686 & 98.71 & n.a. & BMb $^{*}$ \\
2 & 13.91 & n.a. & 1.594 & 0.533 & 1.29 & n.a. & bMB $^{*}$ \\
\hline Total: & & & 106.781 & 41.219 & 100.00 & 0.000 & \\
\hline
\end{tabular}


NMR Spectra and HPLC Chromatographs of $\mathbf{2 e}$

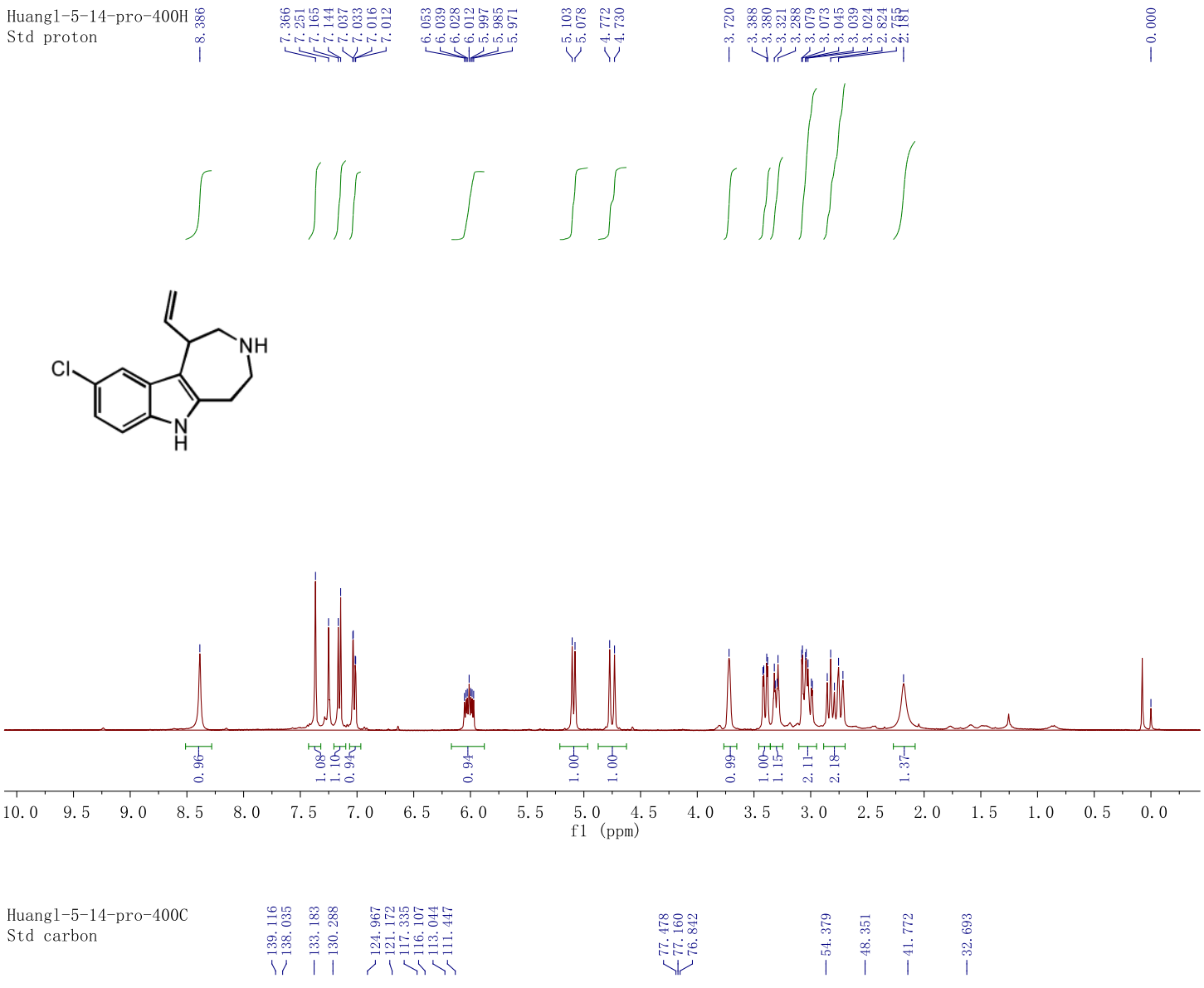

Std carbon $\quad$ li

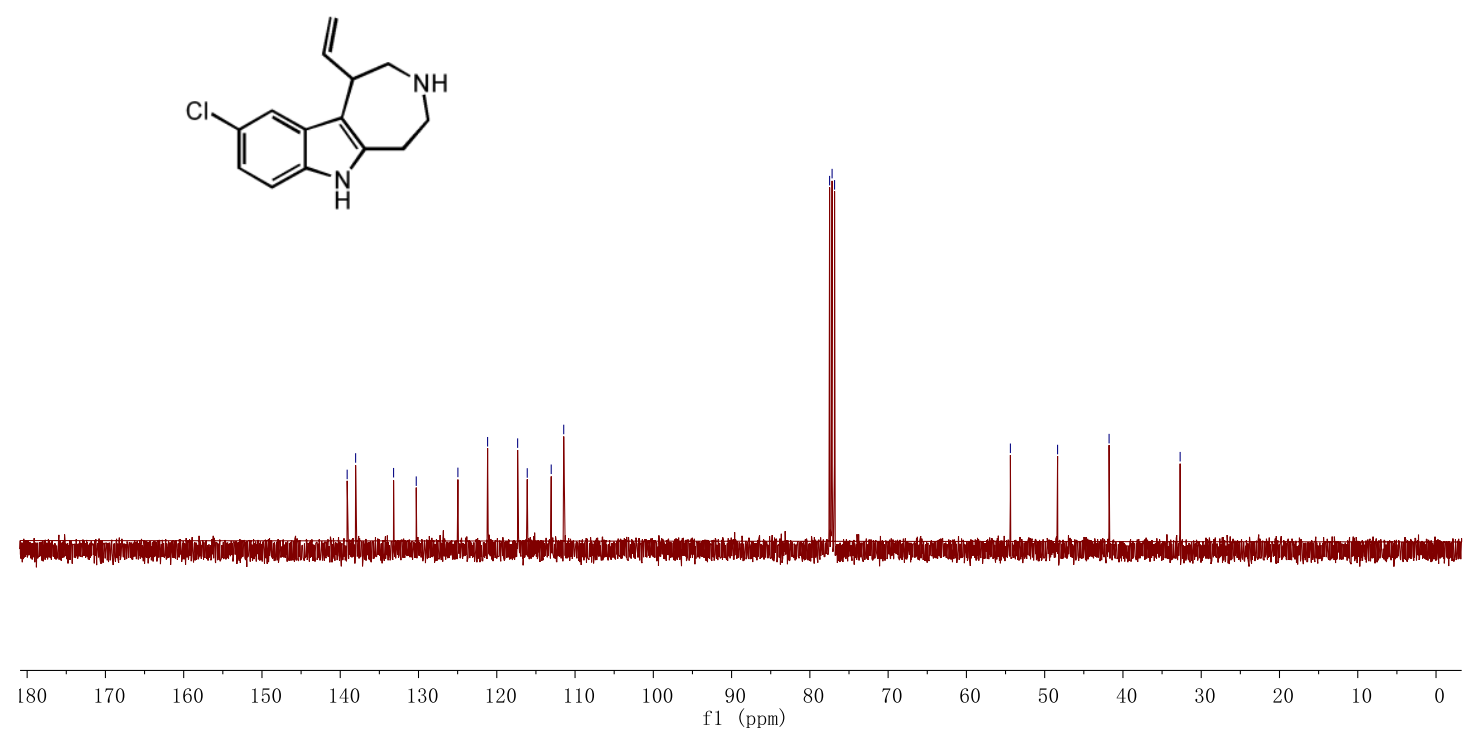




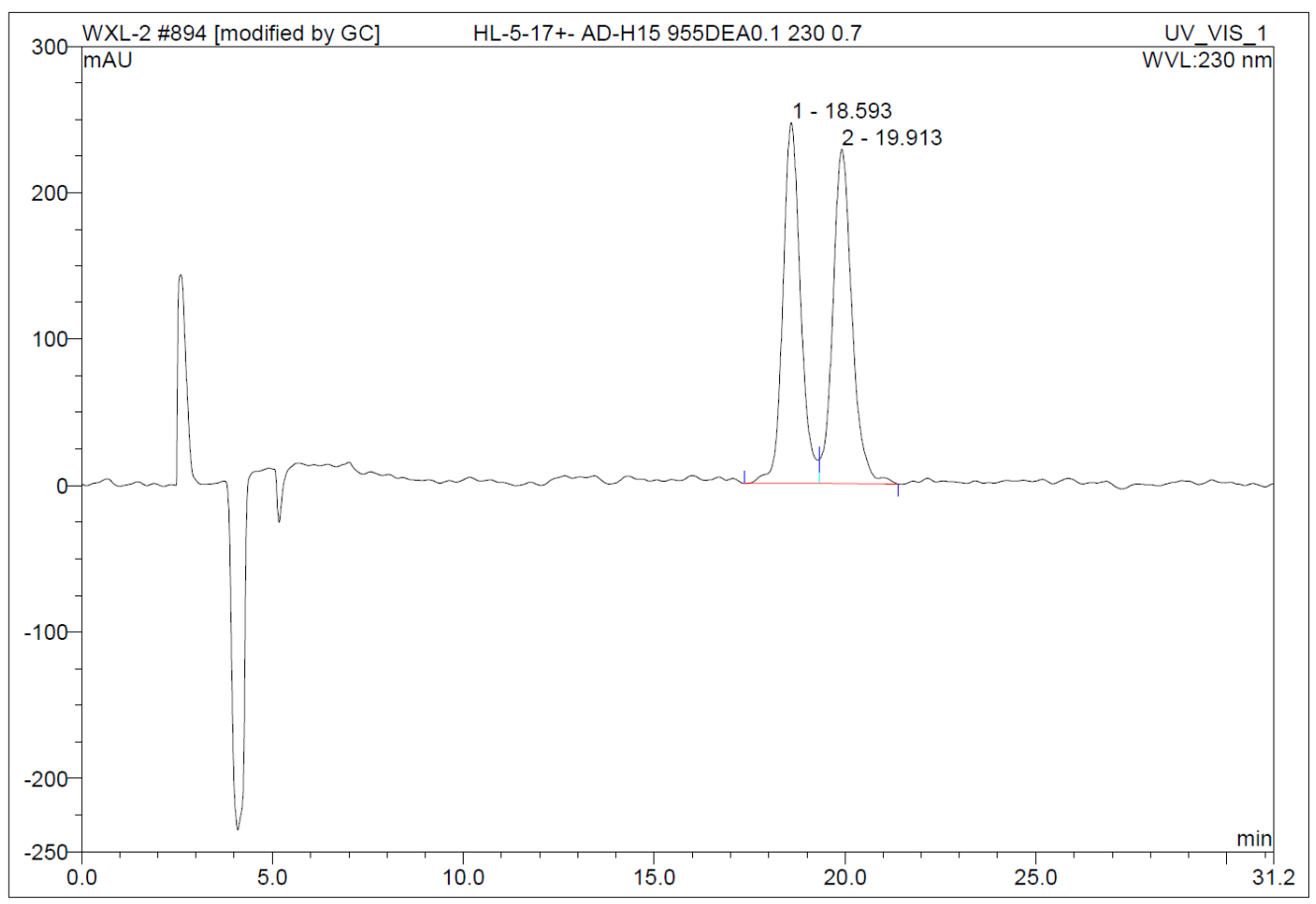

\begin{tabular}{|r|cccrrrr|}
\hline No. & $\begin{array}{c}\text { Ret.Time } \\
\text { min }\end{array}$ & Peak Name & $\begin{array}{c}\text { Height } \\
\text { mAU }\end{array}$ & $\begin{array}{c}\text { Area } \\
\text { mAU*min }\end{array}$ & $\begin{array}{c}\text { Rel.Area } \\
\%\end{array}$ & Amount & Type \\
\hline 1 & 18.59 & n.a. & 246.231 & 131.989 & 49.75 & n.a. & BM $^{*}$ \\
2 & 19.91 & n.a. & 228.239 & 133.307 & 50.25 & n.a. & MB $^{*}$ \\
\hline Total: & & & 474.471 & 265.296 & 100.00 & 0.000 & \\
\hline
\end{tabular}

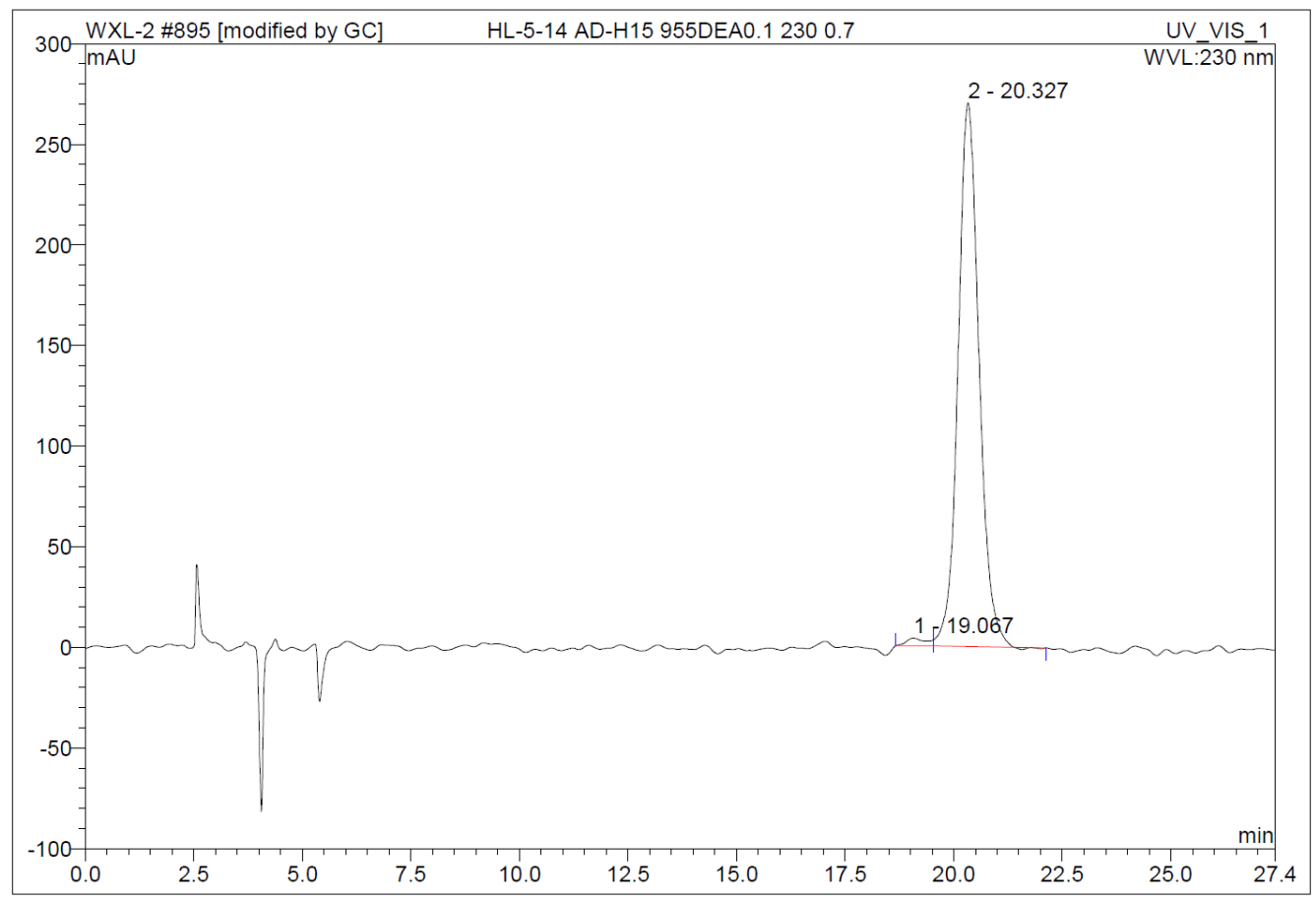

\begin{tabular}{|r|ccrrrrr|}
\hline No. & $\begin{array}{c}\text { Ret.Time } \\
\text { min }\end{array}$ & Peak Name & $\begin{array}{c}\text { Height } \\
\text { mAU }\end{array}$ & $\begin{array}{c}\text { Area } \\
\text { mAU*min }\end{array}$ & $\begin{array}{r}\text { Rel.Area } \\
\%\end{array}$ & Amount & Type \\
\hline 1 & 19.07 & n.a. & 3.764 & 2.001 & 1.30 & n.a. & BM $^{*}$ \\
2 & 20.33 & n.a. & 270.026 & 151.371 & 98.70 & n.a. & MB $^{*}$ \\
\hline Total: & & & 273.790 & 153.371 & 100.00 & 0.000 & \\
\hline
\end{tabular}


NMR Spectra and HPLC Chromatographs of $\mathbf{2 f}$

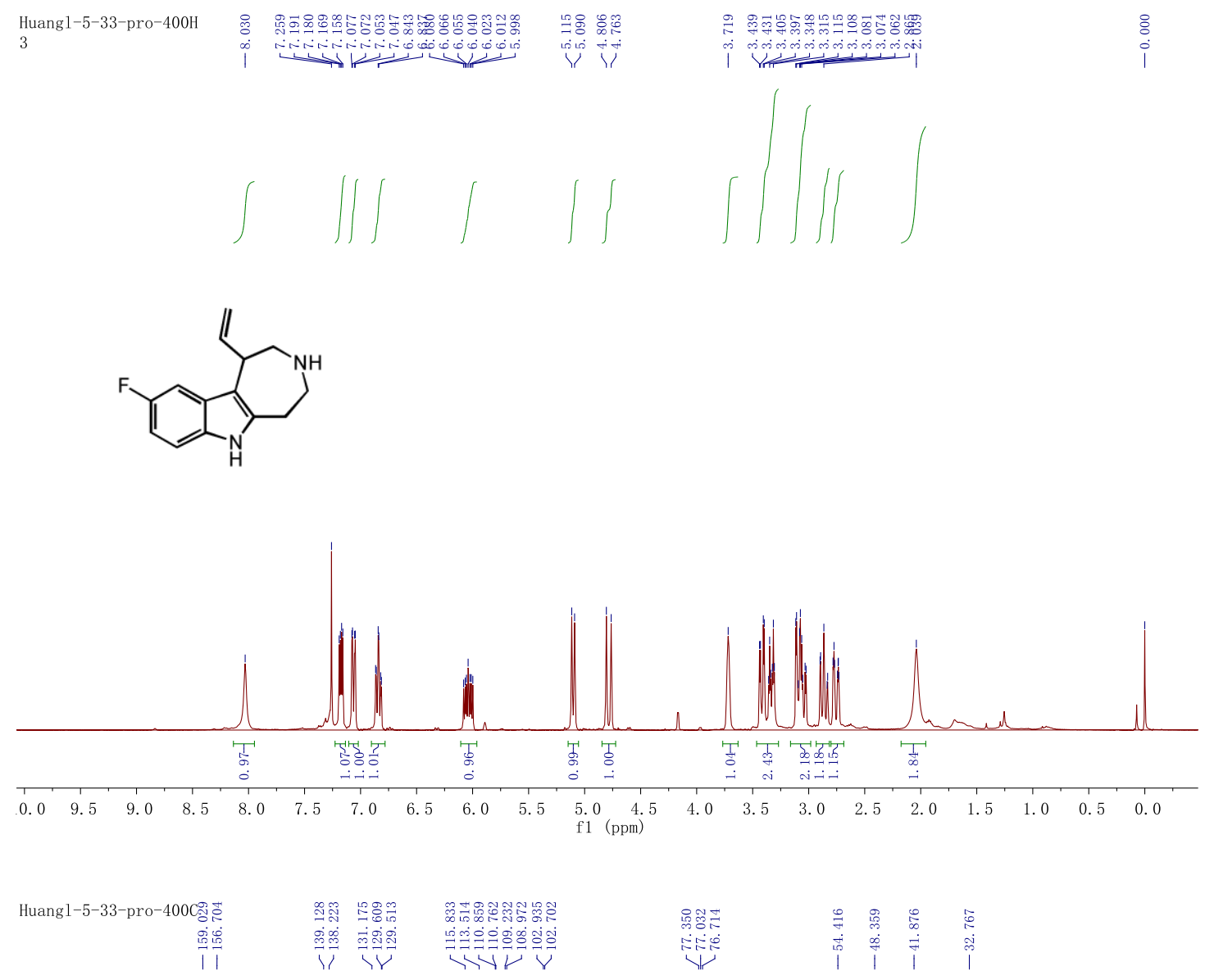<smiles>C=CC1CNCCc2[nH]c3ccc(F)cc3c21</smiles>

$\begin{array}{lllllllllllllllllll}180 & 170 & 160 & 150 & 140 & 130 & 120 & 110 & 100 & 90 & 80 & 70 & 60 & 50 & 40 & 30 & 20 & 10 & 0 \\ \mathrm{f} 1 & (\mathrm{pmm}) & & & \end{array}$ 
<smiles>C=CC1CNCCc2[nH]c3ccc(F)cc3c21</smiles>

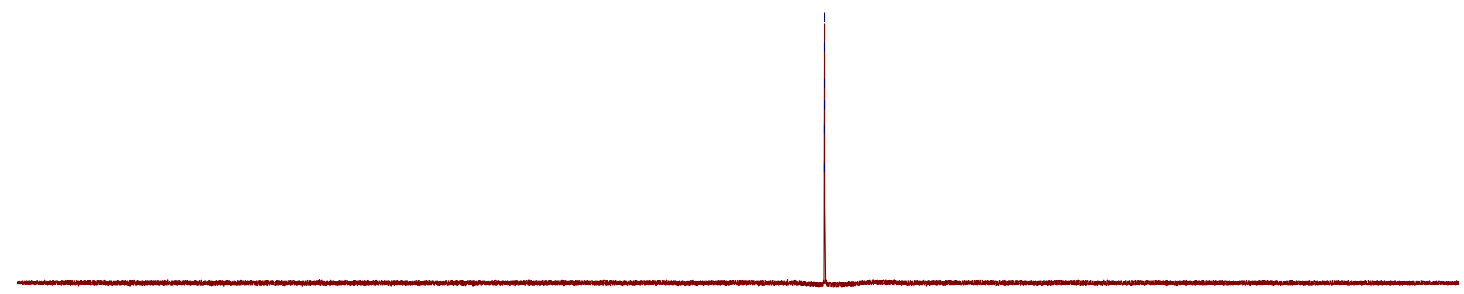

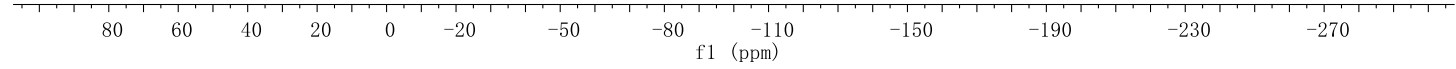

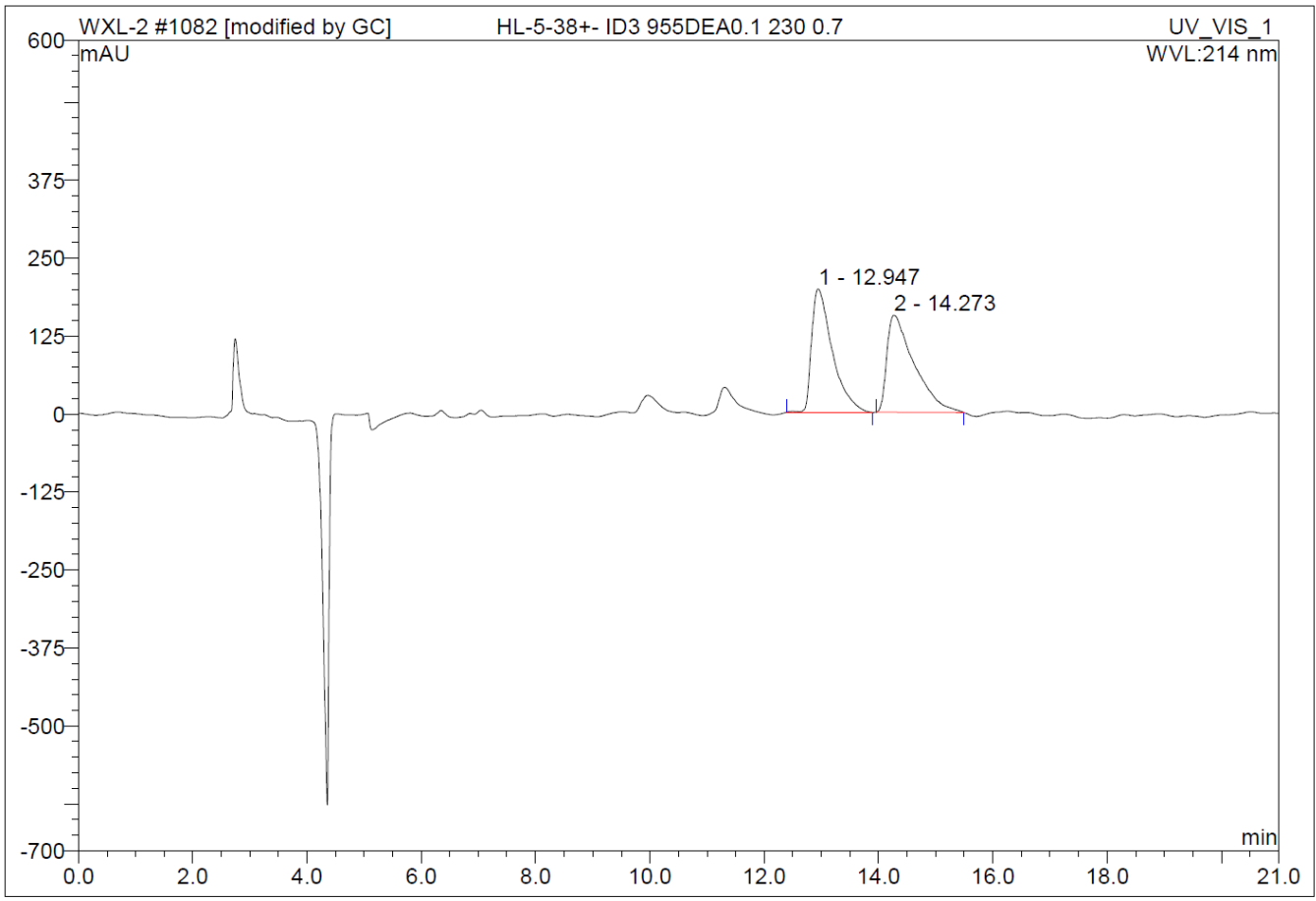

\begin{tabular}{|r|cccrrrr|}
\hline No. & $\begin{array}{c}\text { Ret.Time } \\
\text { min }\end{array}$ & Peak Name & $\begin{array}{c}\text { Height } \\
\text { mAU }\end{array}$ & $\begin{array}{c}\text { Area } \\
\text { mAU*min }\end{array}$ & $\begin{array}{r}\text { Rel.Area } \\
\%\end{array}$ & Amount & Type \\
\hline 1 & 12.95 & n.a. & 197.710 & 83.543 & 49.30 & n.a. & MB $^{*}$ \\
2 & 14.27 & n.a. & 155.433 & 85.909 & 50.70 & n.a. & BMB $^{*}$ \\
\hline Total: & & & 353.144 & 169.452 & 100.00 & 0.000 & \\
\hline
\end{tabular}




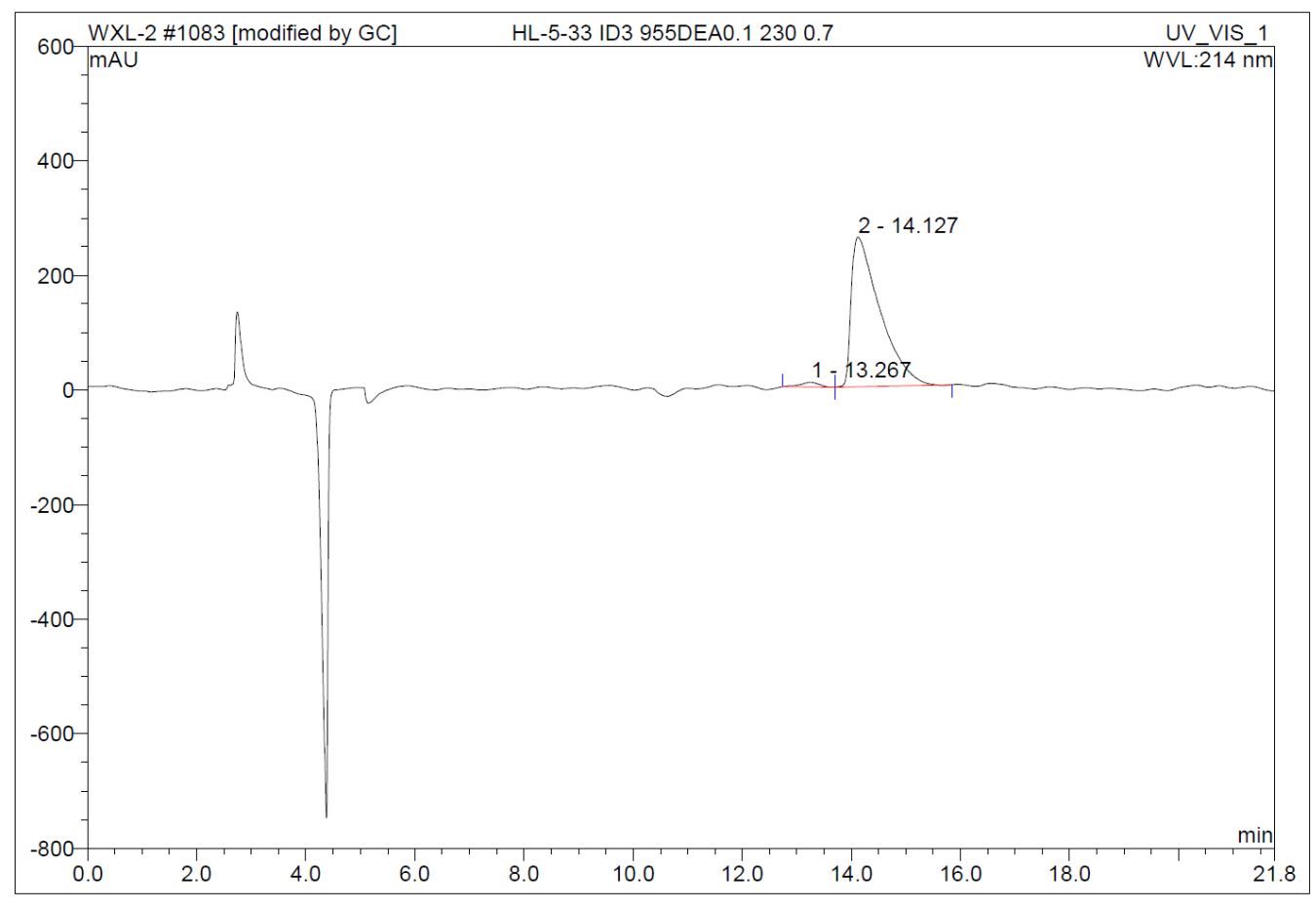

\begin{tabular}{|r|rrrrrrr|}
\hline No. & $\begin{array}{c}\text { Ret.Time } \\
\text { min }\end{array}$ & Peak Name & $\begin{array}{c}\text { Height } \\
\text { mAU }\end{array}$ & $\begin{array}{c}\text { Area } \\
\text { mAU*min }\end{array}$ & $\begin{array}{r}\text { Rel.Area } \\
\%\end{array}$ & Amount & Type \\
\hline 1 & 13.27 & n.a. & 7.954 & 3.097 & 1.90 & n.a. & BMb* $^{*}$ \\
2 & 14.13 & n.a. & 261.165 & 159.624 & 98.10 & n.a. & bMB $^{*}$ \\
\hline Total: & & & 269.119 & 162.722 & 100.00 & 0.000 & \\
\hline
\end{tabular}

NMR Spectra and HPLC Chromatographs of $\mathbf{2 g}$

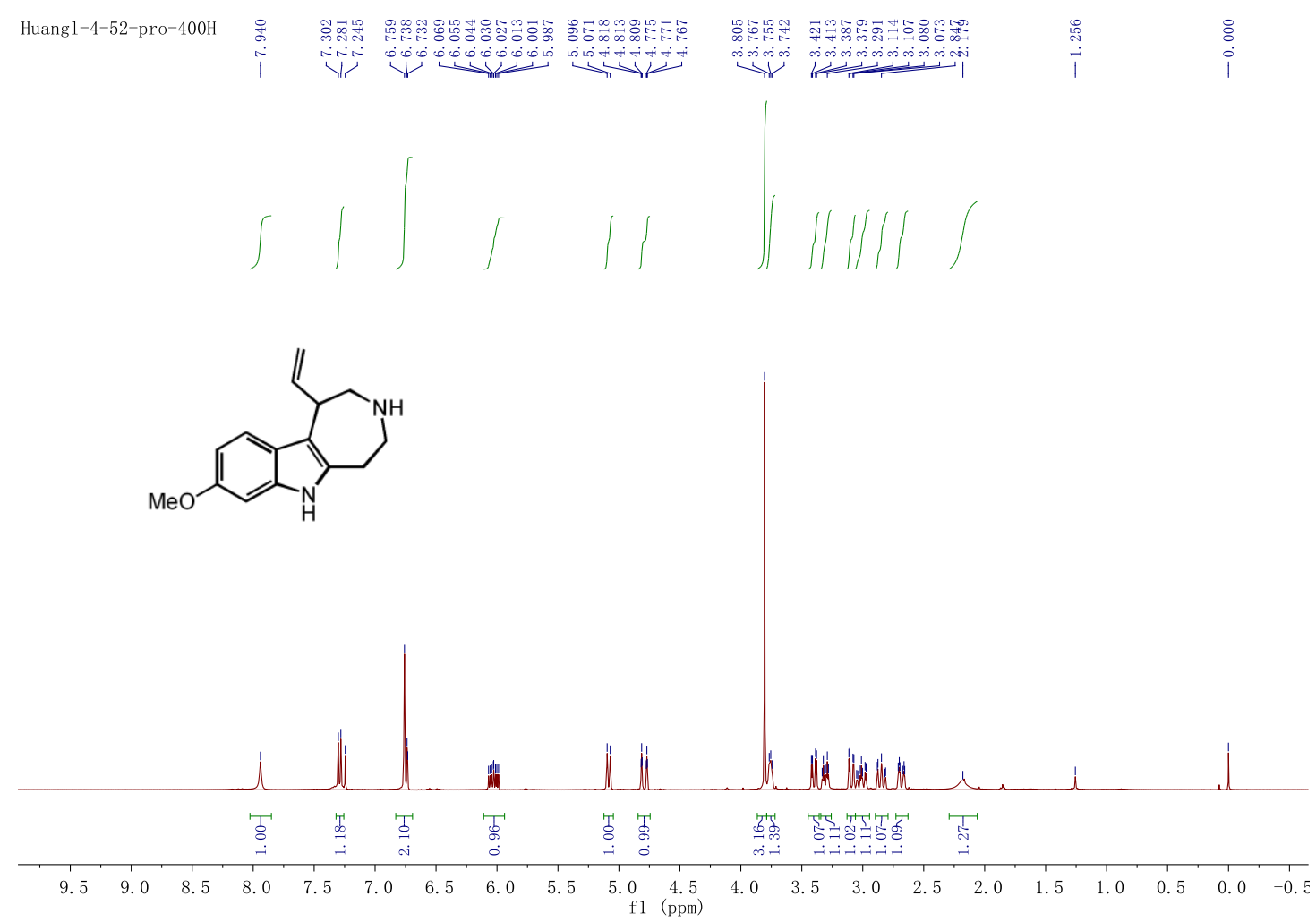



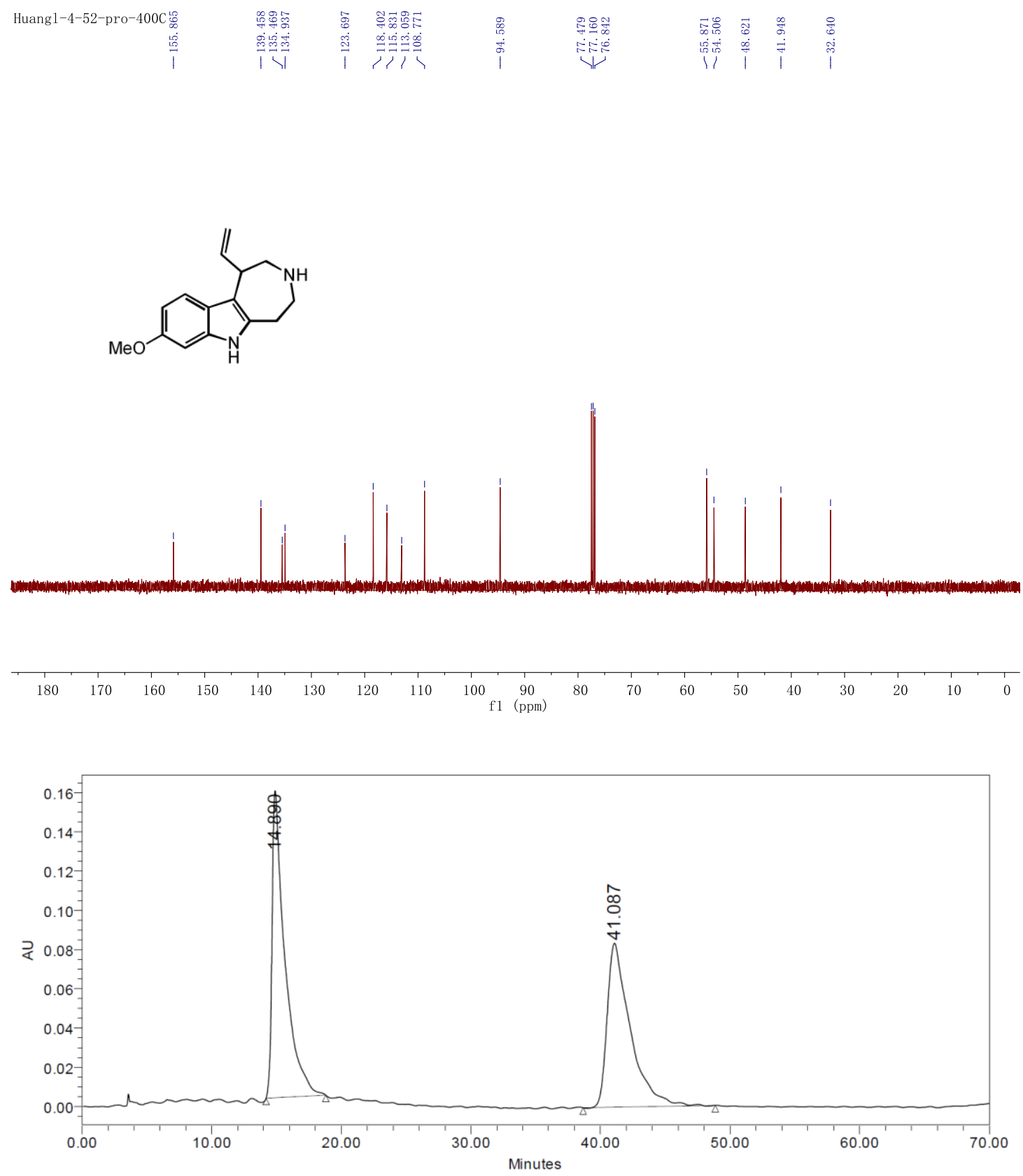

Channel: W2489 ChA; Processed Channel: W2489 ChA 230nm; Injection: 1; Date Acquired: 8/7/2014 2:12:59 PM CST; Result ld: 1334; Processing Method: 42

Peak Name:

\begin{tabular}{|c|r|c|c|r|r|}
\hline & Injection & RT & Area & \% Area & Height \\
\hline 1 & 1 & 41.087 & 10356539 & 49.03 & 83542 \\
\hline 2 & 1 & 14.890 & 10764481 & 50.97 & 156429 \\
\hline Mean & & 27.989 & & & \\
\hline Std. Dev. & & 18.524 & & & \\
\hline \% RSD & & 66.18 & & & \\
\hline
\end{tabular}




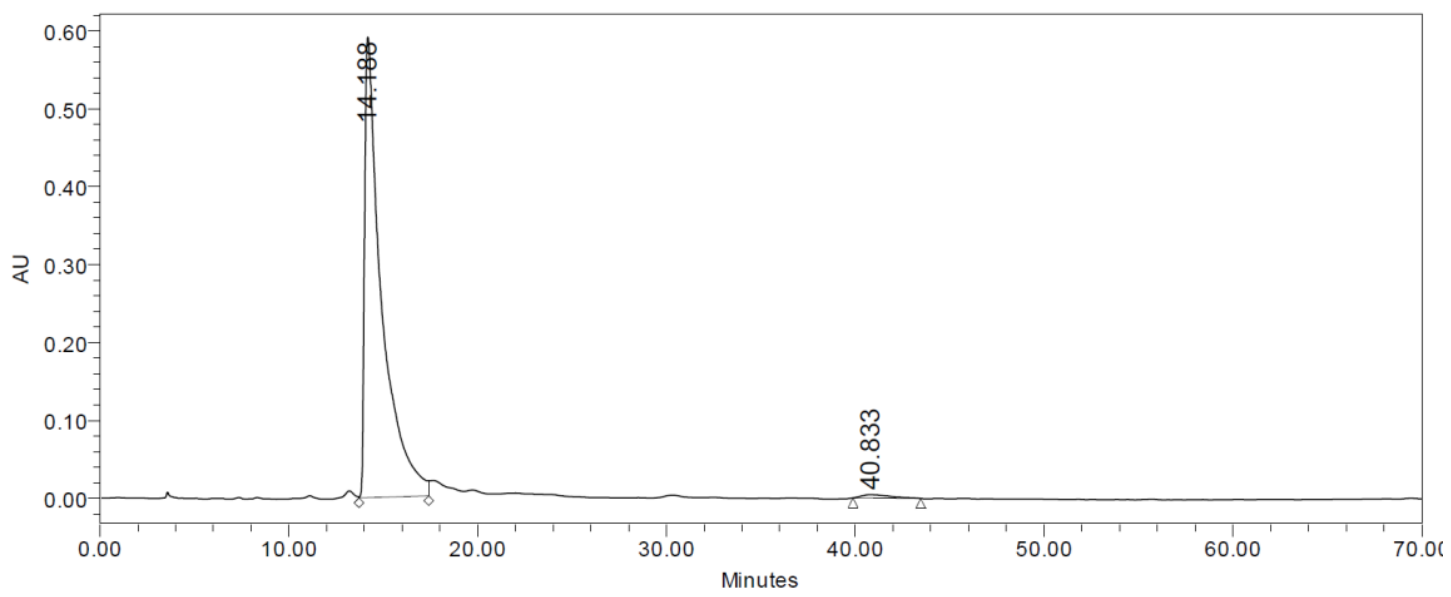

Channel: W2489 ChA; Processed Channel: W2489 ChA 230nm; Injection: 1; Date Acquired: 8/7/2014 3:23:24 PM CST; Result Id: 1517; Processing Method: qwqe

Peak Name:

\begin{tabular}{|c|r|c|c|r|r|}
\hline & Injection & RT & Area & $\%$ Area & Height \\
\hline 1 & 1 & 40.833 & 468793 & 1.25 & 4152 \\
\hline 2 & 1 & 14.188 & 37134723 & 98.75 & 591862 \\
\hline Mean & & 27.511 & & & \\
\hline Std. Dev. & & 18.841 & & & \\
\hline \% RSD & & 68.49 & & & \\
\hline
\end{tabular}

NMR Spectra and HPLC Chromatographs of $\mathbf{2 h}$

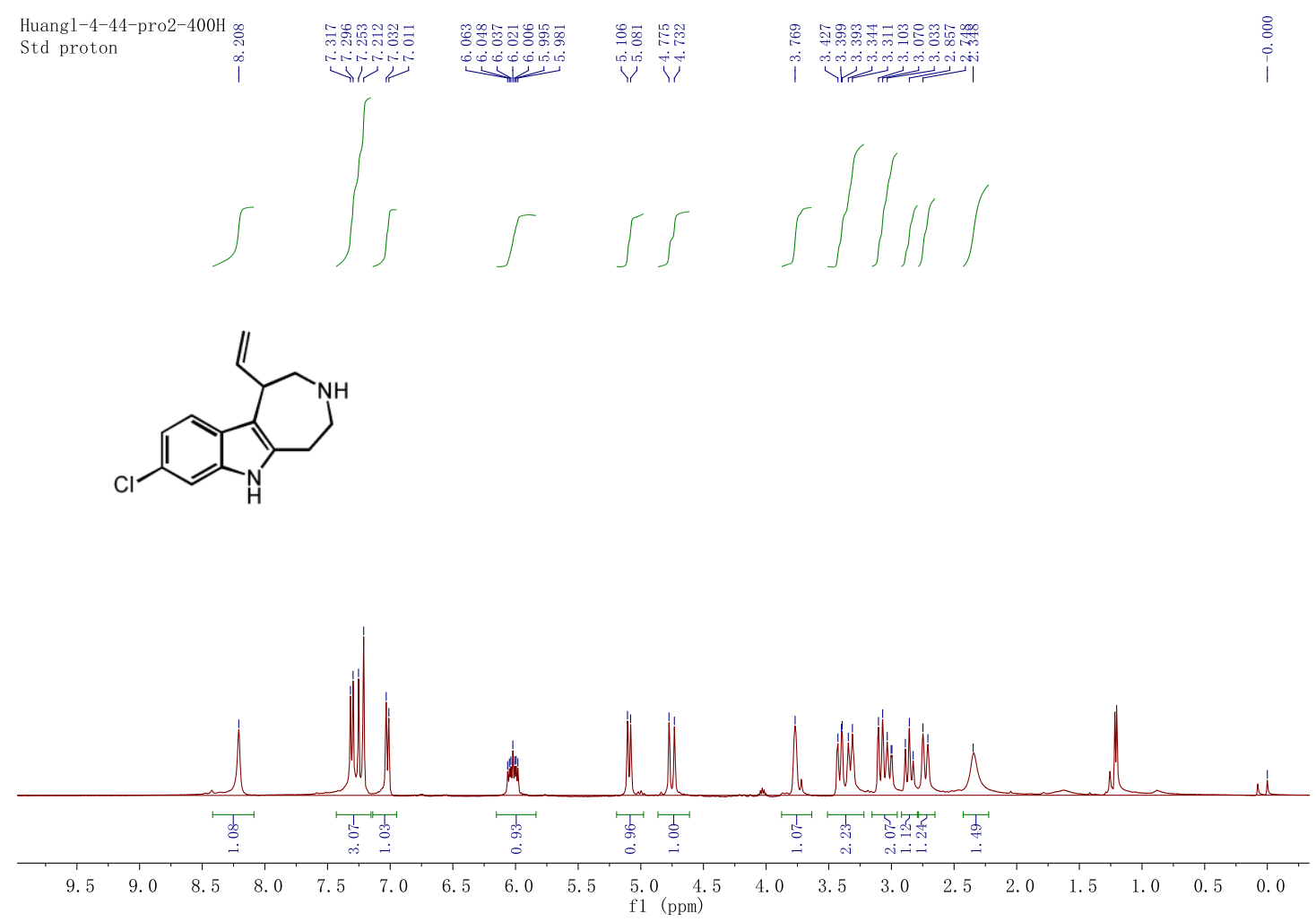




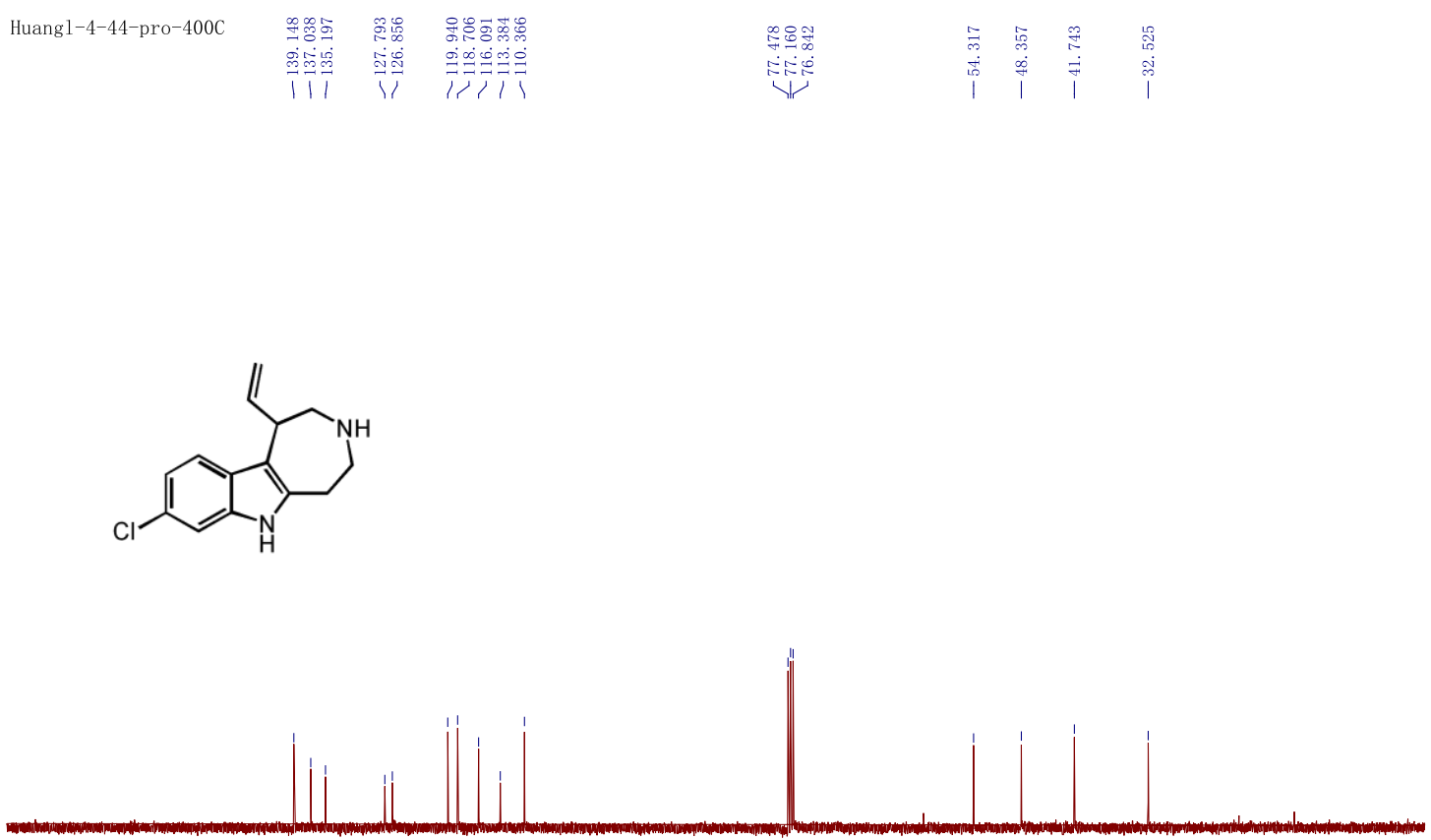

\begin{tabular}{|c|c|c|c|c|c|c|c|c|c|c|c|c|c|c|c|}
\hline 170 & 160 & 150 & 140 & 130 & 120 & 110 & 100 & $\begin{array}{l}90 \\
\mathrm{f} 1 \text { (ppm) }\end{array}$ & 70 & 60 & 50 & 40 & 30 & 20 & 10 \\
\hline
\end{tabular}

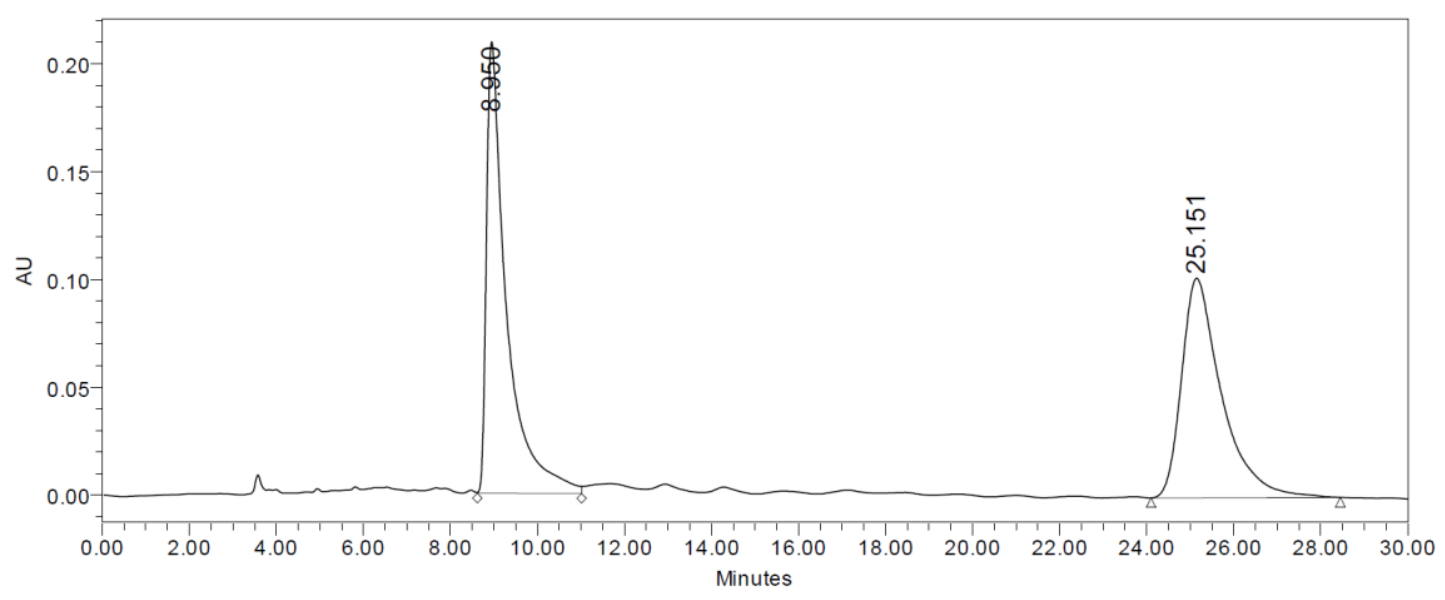

Channel: W2489 ChA; Processed Channel: W2489 ChA 230nm; Injection: 1; Date Acquired: 8/7/2014 2:56:51 AM CST; Result Id: 1513; Processing Method: qw

Peak Name:

\begin{tabular}{|c|r|r|c|r|c|}
\hline & Injection & RT & Area & $\%$ Area & Height \\
\hline 1 & 1 & 25.151 & 6479383 & 49.10 & 101736 \\
\hline 2 & 1 & 8.950 & 6716106 & 50.90 & 209382 \\
\hline Mean & & 17.050 & & & \\
\hline Std. Dev. & & 11.456 & & & \\
\hline \% RSD & & 67.19 & & & \\
\hline
\end{tabular}




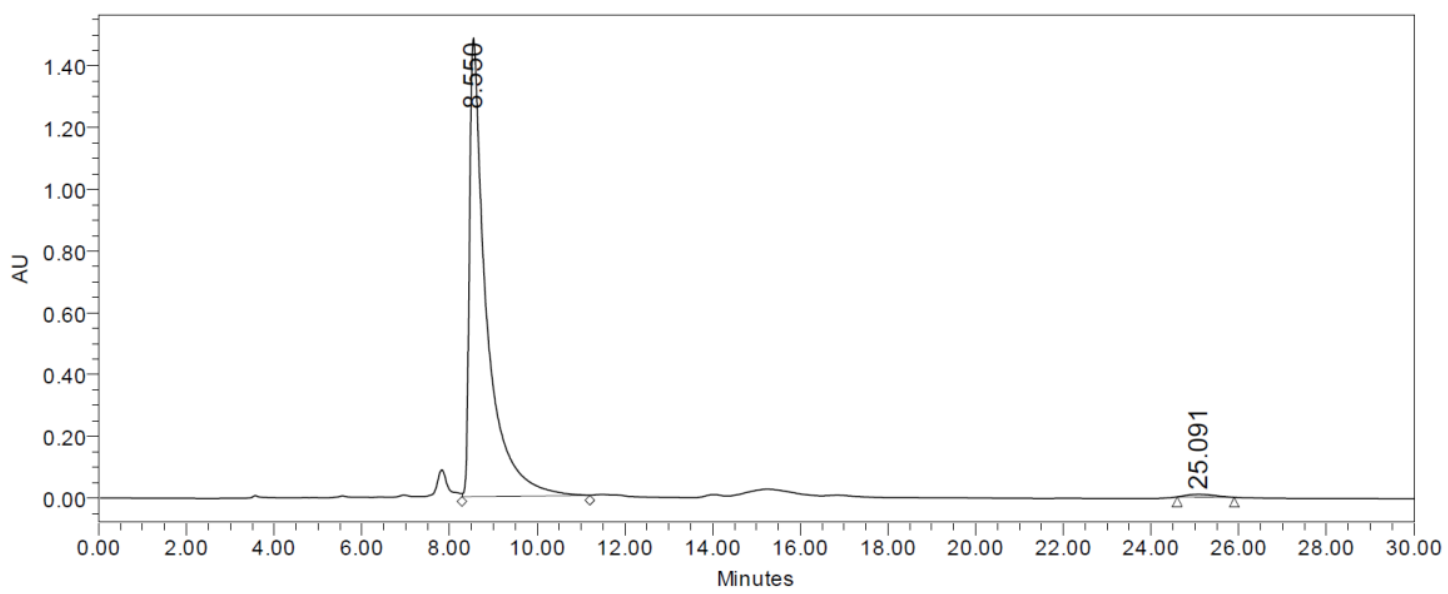

Channel: W2489 ChA; Processed Channel: W2489 ChA 230nm; Injection: 1; Date Acquired: 8/7/2014 3:27:14 AM CST; Result ld: 1515; Processing Method: qe

Peak Name:

\begin{tabular}{|c|r|r|r|r|r|}
\hline & Injection & RT & Area & $\%$ Area & Height \\
\hline 1 & 1 & 25.091 & 377157 & 0.94 & 8705 \\
\hline 2 & 1 & 8.550 & 39605331 & 99.06 & 1484702 \\
\hline Mean & & 16.820 & & & \\
\hline Std. Dev. & & 11.696 & & & \\
\hline \% RSD & & 69.54 & & & \\
\hline
\end{tabular}

NMR Spectra and HPLC Chromatographs of $\mathbf{2 i}$

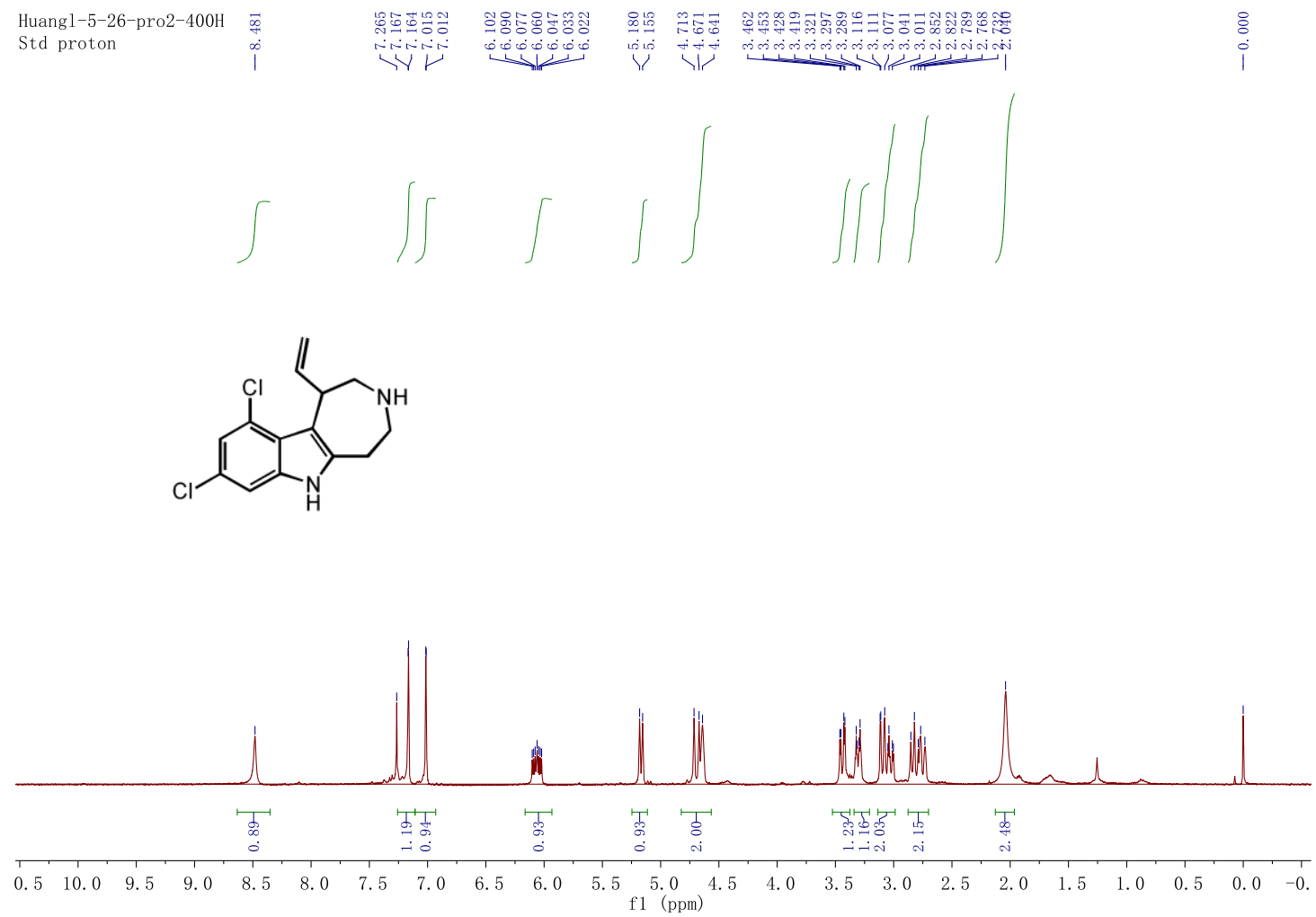


<smiles>C=CC1CNCCc2[nH]c3cc(Cl)cc(Cl)c3c21</smiles>

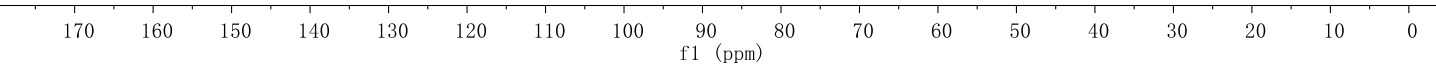

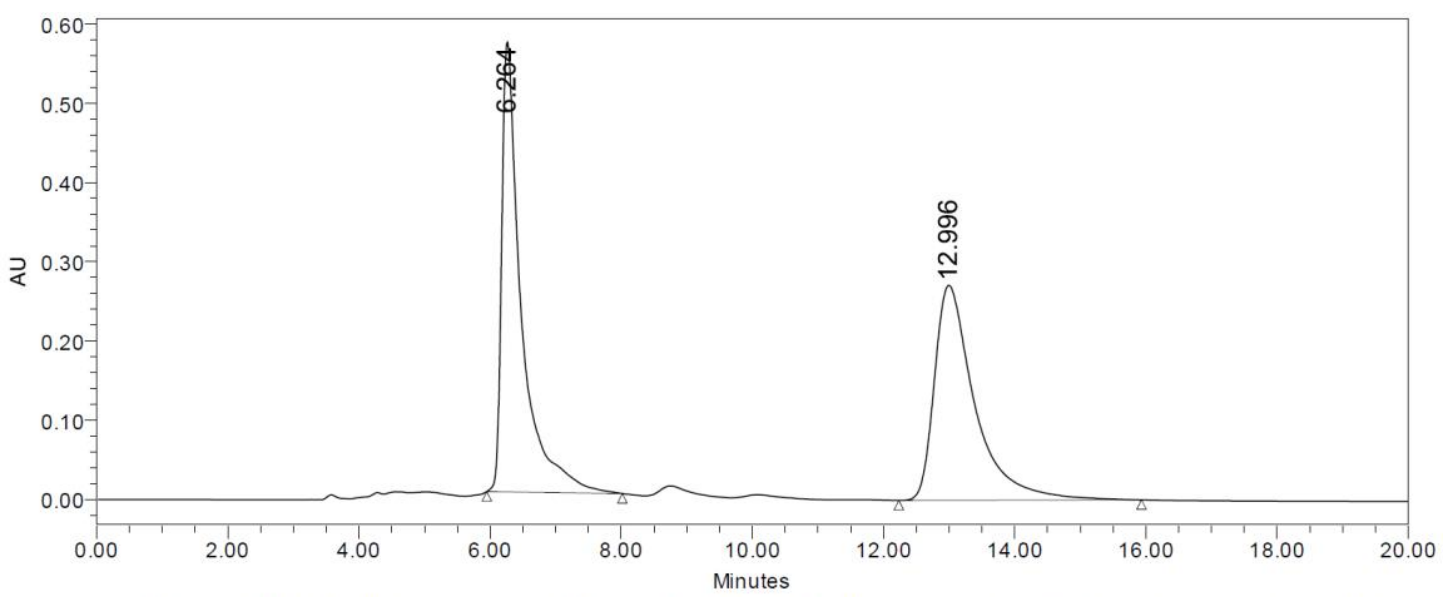

Channel: W2489 ChA; Processed Channel: W2489 ChA 230nm; Injection: 1; Date Acquired: 6/25/2014 11:49:15 PM CST; Result Id: 1256; Processing Method: huangl_5_29_rac

Peak Name:

\begin{tabular}{|c|r|c|c|r|c|}
\hline & Injection & RT & Area & $\%$ Area & Height \\
\hline 1 & 1 & 12.996 & 11607799 & 49.49 & 271287 \\
\hline 2 & 1 & 6.264 & 11849089 & 50.51 & 567955 \\
\hline Mean & & 9.630 & & & \\
\hline Std. Dev. & & 4.760 & & & \\
\hline \% RSD & & 49.43 & & & \\
\hline
\end{tabular}




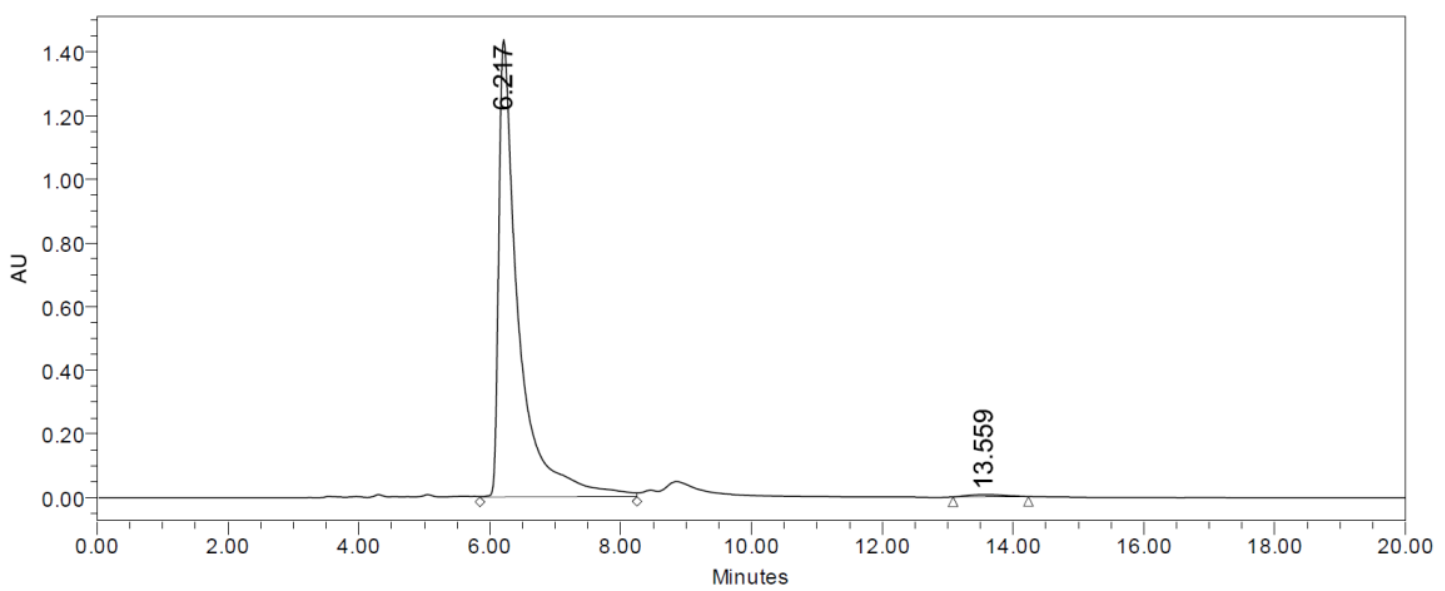

Channel: W2489 ChA; Processed Channel: W2489 ChA 230nm; Injection: 1; Date Acquired: 6/26/2014 12:09:38 AM CST; Result Id: 1258; Processing Method: 22

\begin{tabular}{|c|r|r|r|r|r|}
\multicolumn{7}{|c|}{ Peak Name: } \\
\hline & Injection & RT & Area & $\%$ Area & Height \\
\hline 1 & 1 & 13.559 & 276010 & 0.91 & 7562 \\
\hline 2 & 1 & 6.217 & 30216668 & 99.09 & 1437142 \\
\hline Mean & & 9.888 & & & \\
\hline Std. Dev. & & 5.192 & & & \\
\hline \% RSD & & 52.51 & & & \\
\hline
\end{tabular}

NMR Spectra and HPLC Chromatographs of $\mathbf{2} \mathbf{j}$

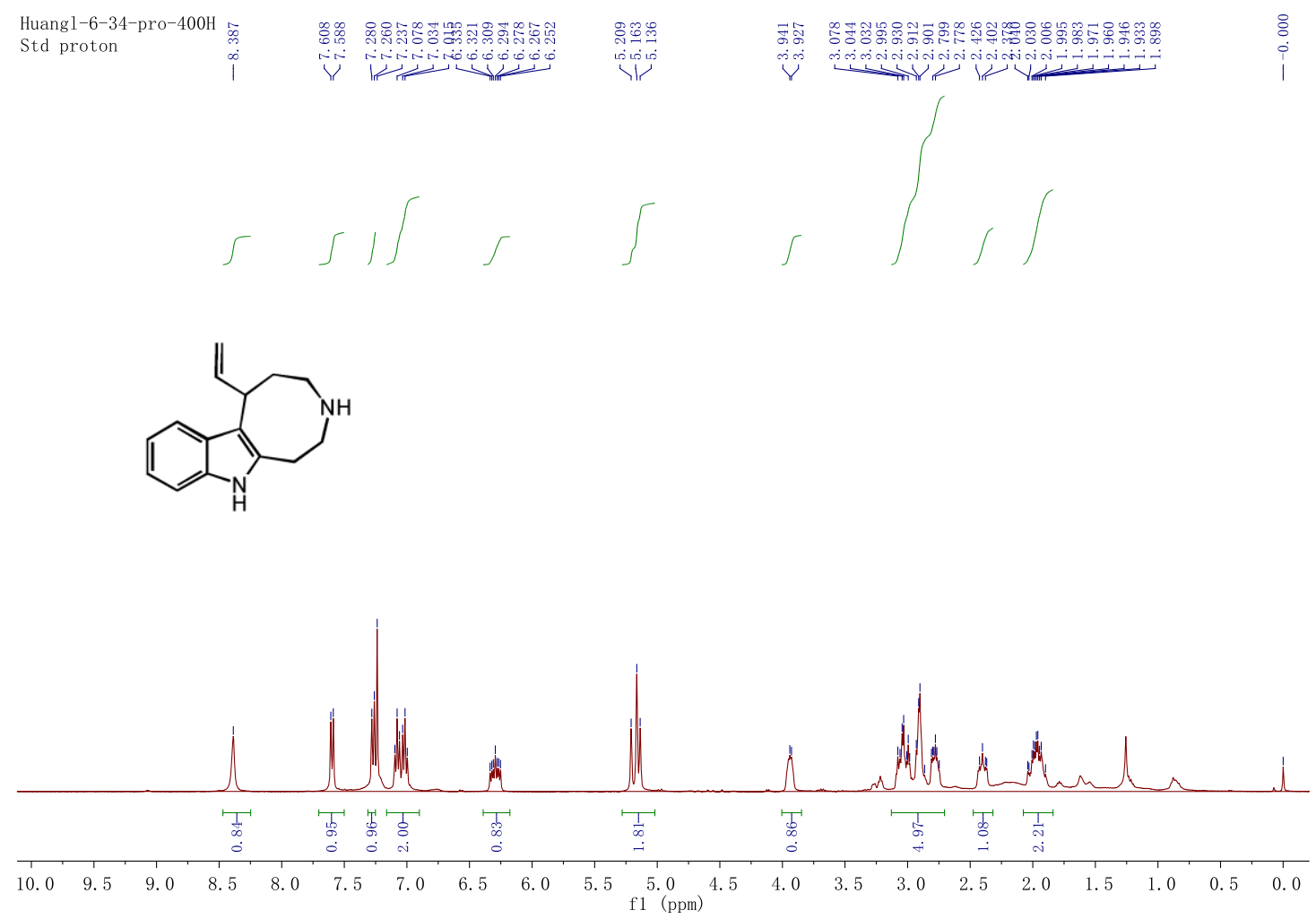


<smiles>C=CC1CCNCCc2[nH]c3ccccc3c21</smiles>

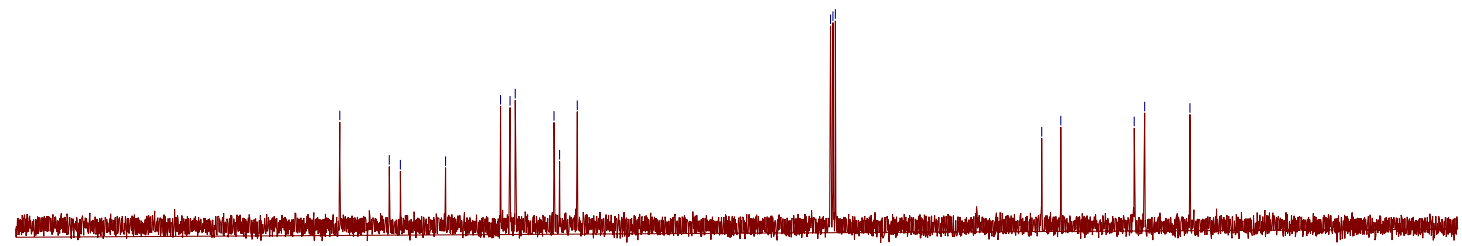

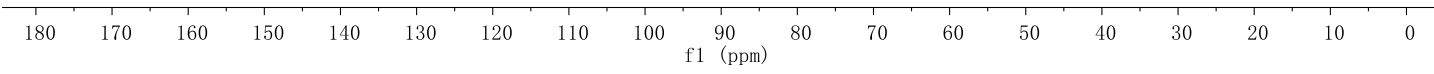

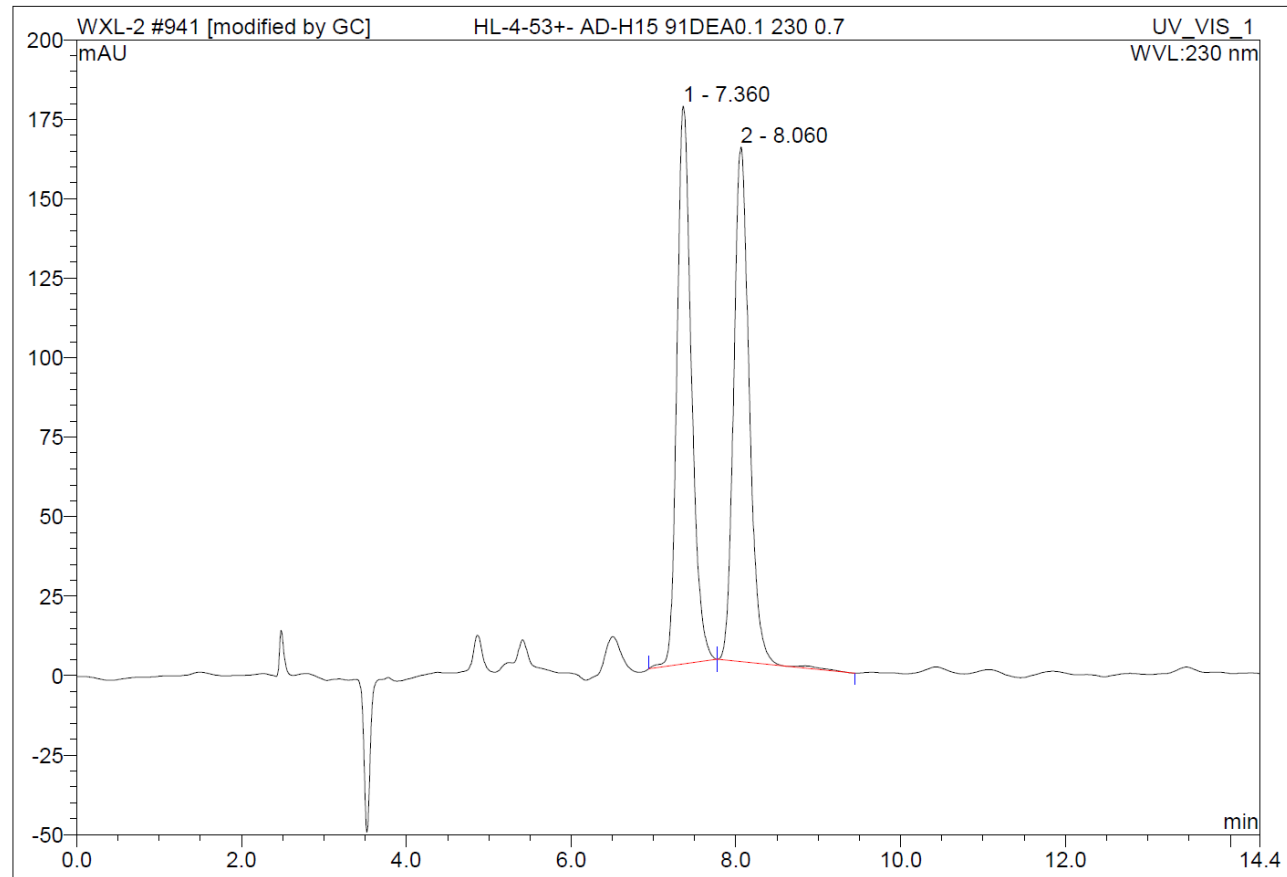

\begin{tabular}{|r|cccrrrr|}
\hline No. & $\begin{array}{c}\text { Ret.Time } \\
\text { min }\end{array}$ & Peak Name & $\begin{array}{c}\text { Height } \\
\text { mAU }\end{array}$ & $\begin{array}{c}\text { Area } \\
\text { mAU*min }\end{array}$ & $\begin{array}{c}\text { Rel.Area } \\
\%\end{array}$ & Amount & Type \\
\hline 1 & 7.36 & n.a. & 175.417 & 35.876 & 50.19 & n.a. & BMb $^{*}$ \\
2 & 8.06 & n.a. & 161.794 & 35.609 & 49.81 & n.a. & bMB $^{*}$ \\
\hline Total: & & & 337.211 & 71.484 & 100.00 & 0.000 & \\
\hline
\end{tabular}




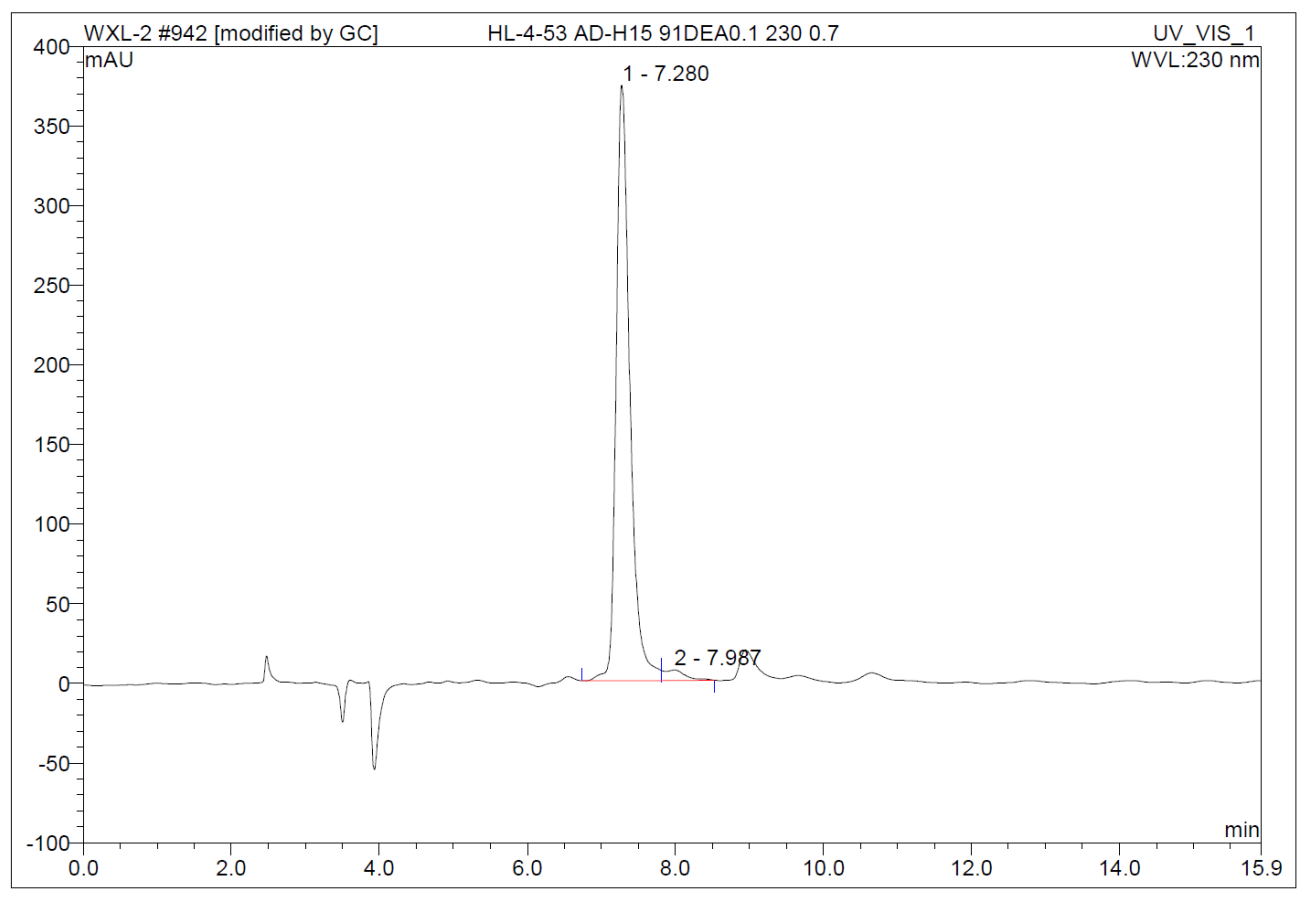

\begin{tabular}{|c|c|c|c|c|c|c|c|}
\hline No. & $\begin{array}{l}\text { Ret.Time } \\
\text { min }\end{array}$ & Peak Name & $\begin{array}{c}\text { Height } \\
\text { mAU }\end{array}$ & $\begin{array}{c}\text { Area } \\
\text { mAU*min }\end{array}$ & $\begin{array}{c}\text { Rel.Area } \\
\%\end{array}$ & Amount & Type \\
\hline 1 & 7.28 & n.a. & 373.737 & 80.676 & 97.21 & n.a. & $\mathrm{BM}^{*}$ \\
\hline 2 & 7.99 & n.a. & 6.643 & 2.311 & 2.79 & n.a & $\mathrm{MB}^{*}$ \\
\hline Total: & & & 380.380 & 82.987 & 100.00 & 0.000 & \\
\hline
\end{tabular}

NMR Spectra and HPLC Chromatographs of $\mathbf{2 k}$

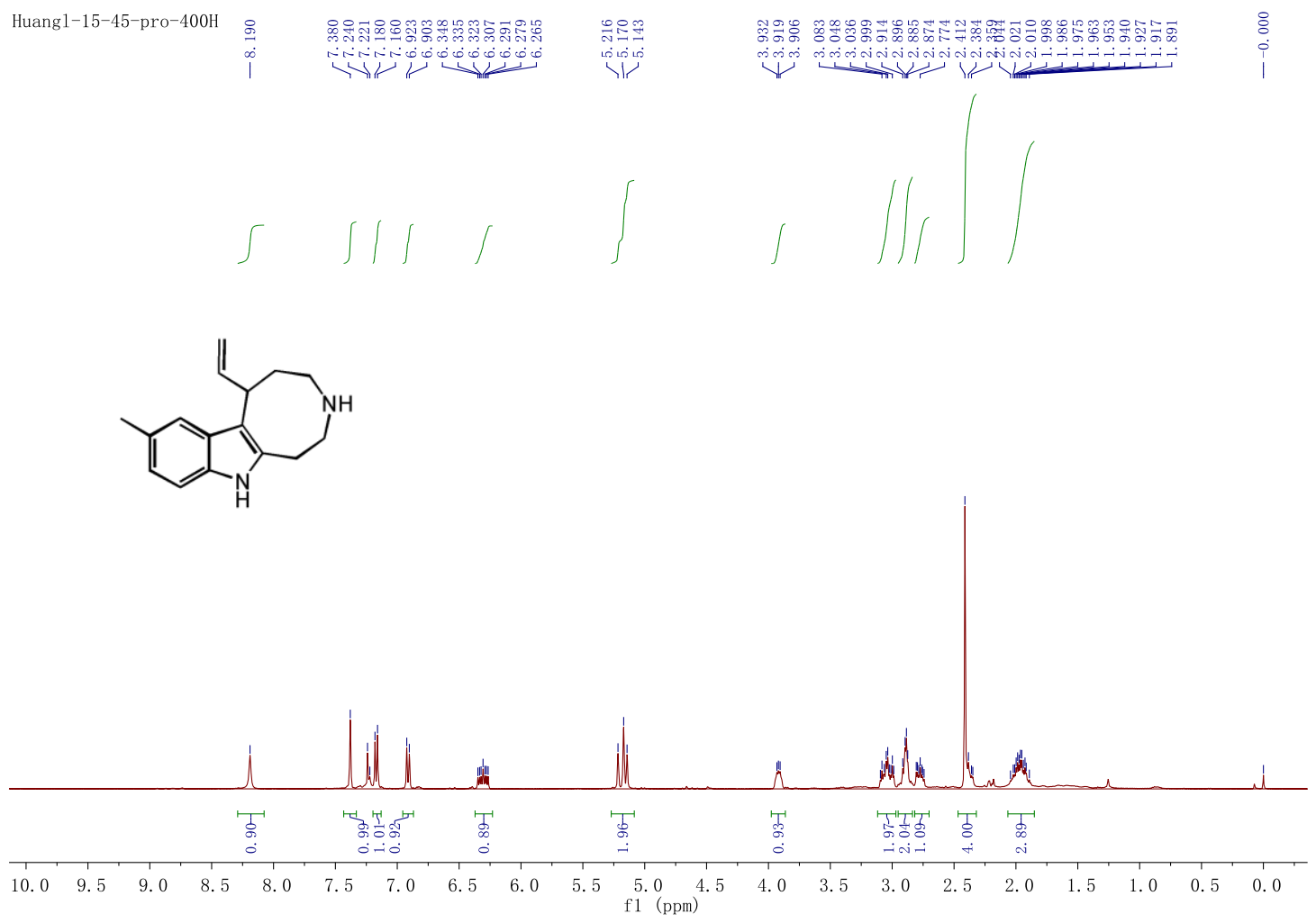


<smiles>C=CC1CCNCCc2[nH]c3ccc(C)cc3c21</smiles>

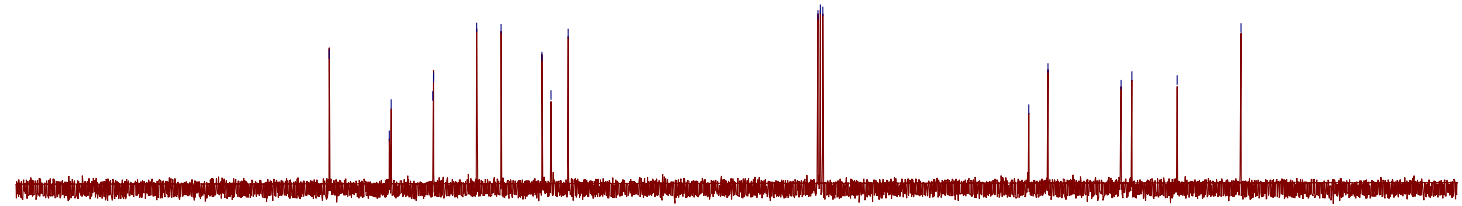

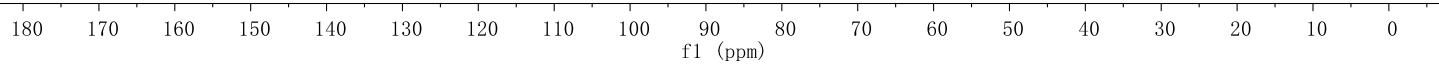

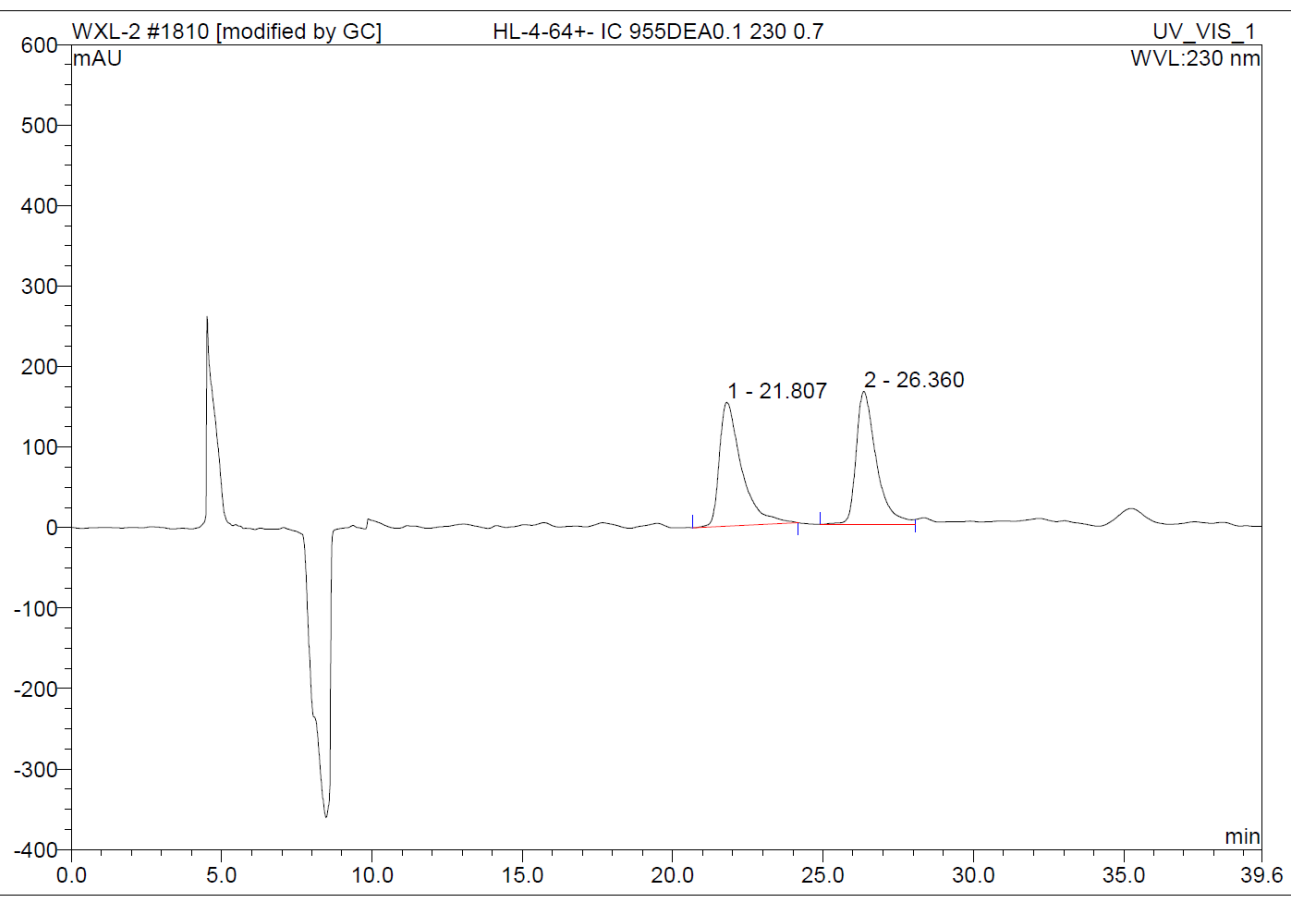

\begin{tabular}{|r|ccccccc|}
\hline No. & $\begin{array}{c}\text { Ret.Time } \\
\text { min }\end{array}$ & Peak Name & $\begin{array}{c}\text { Height } \\
\text { mAU }\end{array}$ & $\begin{array}{c}\text { Area } \\
\text { mAU*min }\end{array}$ & $\begin{array}{c}\text { Rel.Area } \\
\%\end{array}$ & Amount & Type \\
\hline 1 & 21.81 & n.a. & 153.617 & 131.930 & 50.51 & n.a. & BMB $^{*}$ \\
2 & 26.36 & n.a. & 165.085 & 129.247 & 49.49 & n.a. & BM $^{*}$ \\
\hline Total: & & & 318.702 & 261.177 & 100.00 & 0.000 & \\
\hline
\end{tabular}




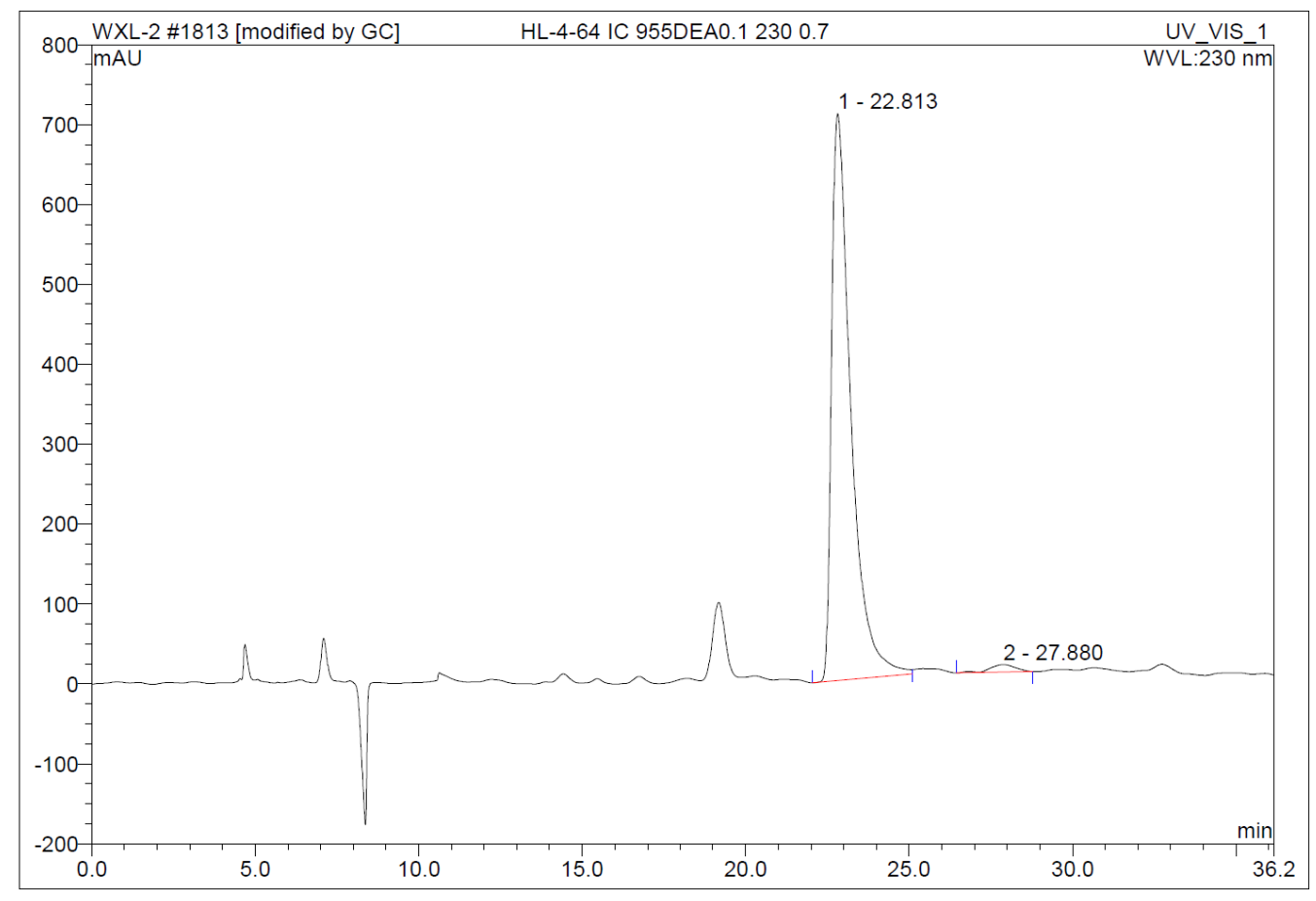

\begin{tabular}{|r|ccrrrrr|}
\hline No. & $\begin{array}{c}\text { Ret.Time } \\
\text { min }\end{array}$ & Peak Name & $\begin{array}{c}\text { Height } \\
\text { mAU }\end{array}$ & $\begin{array}{c}\text { Area } \\
\text { mAU*min }\end{array}$ & $\begin{array}{r}\text { Rel.Area } \\
\%\end{array}$ & Amount & Type \\
\hline 1 & 22.81 & n.a. & 709.129 & 485.245 & 98.25 & n.a. & BM $^{*}$ \\
2 & 27.88 & n.a. & 9.426 & 8.660 & 1.75 & n.a. & BMB $^{*}$ \\
\hline Total: & & & 718.555 & 493.905 & 100.00 & 0.000 & \\
\hline
\end{tabular}

NMR Spectra and HPLC Chromatographs of $2 \mathbf{l}$

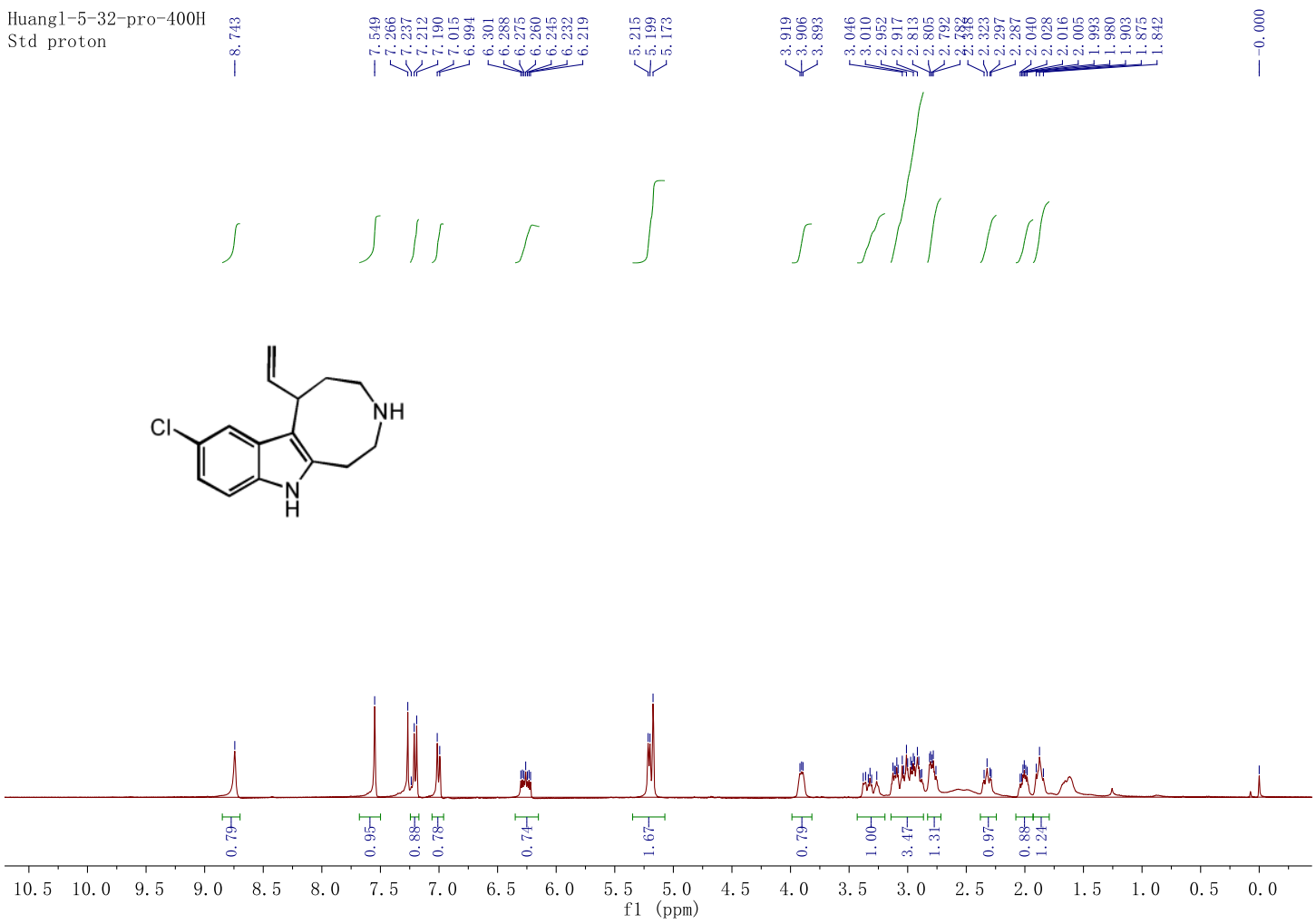


<smiles>C=CC1CCNCCc2[nH]c3ccc(Cl)cc3c21</smiles>
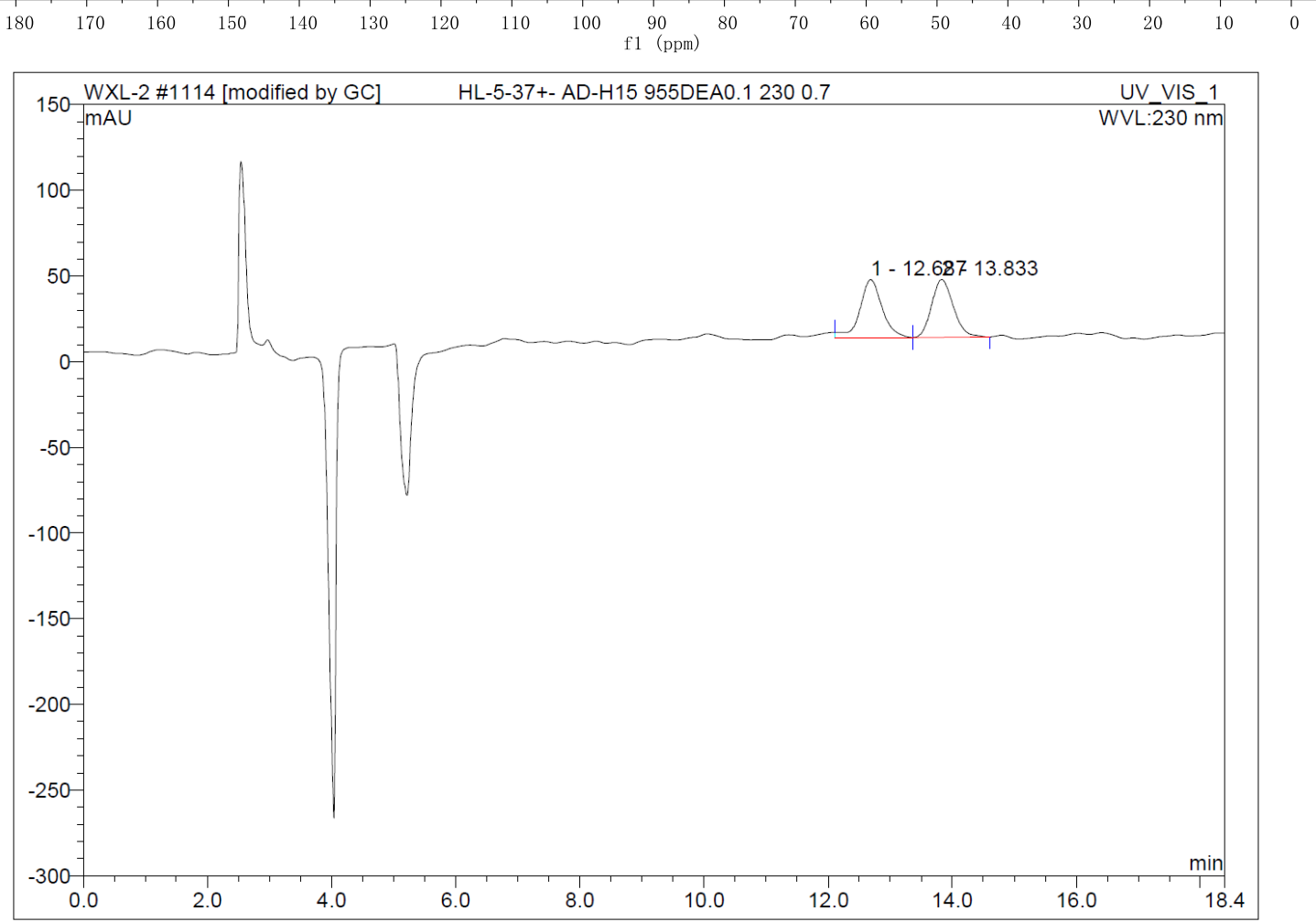

\begin{tabular}{|r|cccrrrr|}
\hline No. & $\begin{array}{c}\text { Ret.Time } \\
\text { min }\end{array}$ & Peak Name & $\begin{array}{c}\text { Height } \\
\text { mAU }\end{array}$ & $\begin{array}{r}\text { Area } \\
\text { mAU*min }\end{array}$ & $\begin{array}{c}\text { Rel.Area } \\
\%\end{array}$ & Amount & Type \\
\hline 1 & 12.69 & n.a. & 33.987 & 14.108 & 50.73 & n.a. & $\mathrm{MB}^{*}$ \\
2 & 13.83 & n.a. & 33.880 & 13.701 & 49.27 & n.a. & BMB $^{*}$ \\
\hline Total: & & & 67.867 & 27.809 & 100.00 & 0.000 & \\
\hline
\end{tabular}




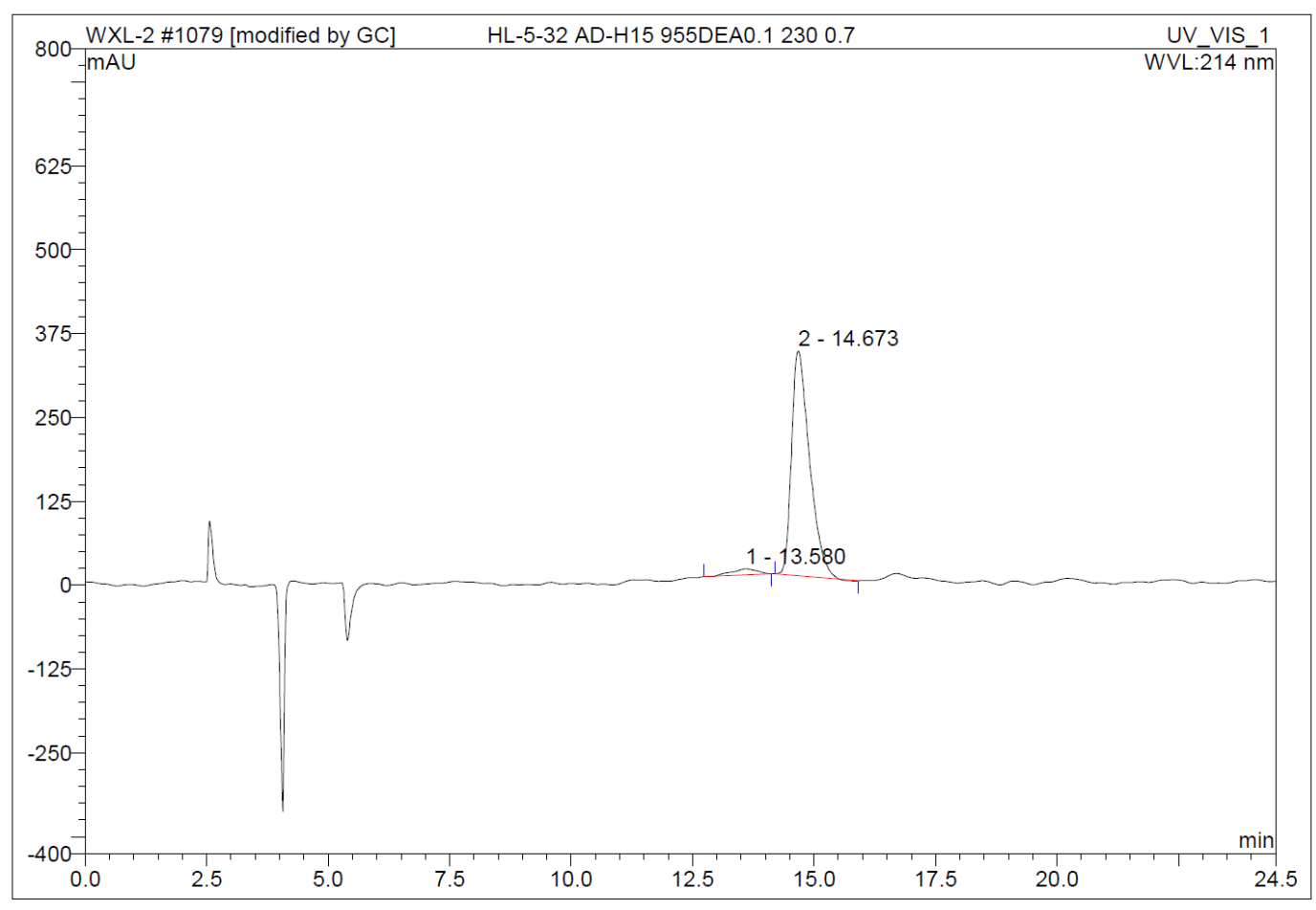

\begin{tabular}{|r|ccrrrrr|}
\hline No. & $\begin{array}{c}\text { Ret.Time } \\
\text { min }\end{array}$ & Peak Name & $\begin{array}{c}\text { Height } \\
\text { mAU }\end{array}$ & $\begin{array}{c}\text { Area } \\
\text { mAU*min }\end{array}$ & $\begin{array}{r}\text { Rel.Area } \\
\%\end{array}$ & Amount & Type \\
\hline 1 & 13.58 & n.a. & 8.902 & 5.364 & 3.60 & n.a. & BMB $^{*}$ \\
2 & 14.67 & n.a. & 334.604 & 143.616 & 96.40 & n.a. & BMB $^{*}$ \\
\hline Total: & & & 343.506 & 148.980 & 100.00 & 0.000 & \\
\hline
\end{tabular}

NMR Spectra and HPLC Chromatographs of $\mathbf{2 m}$
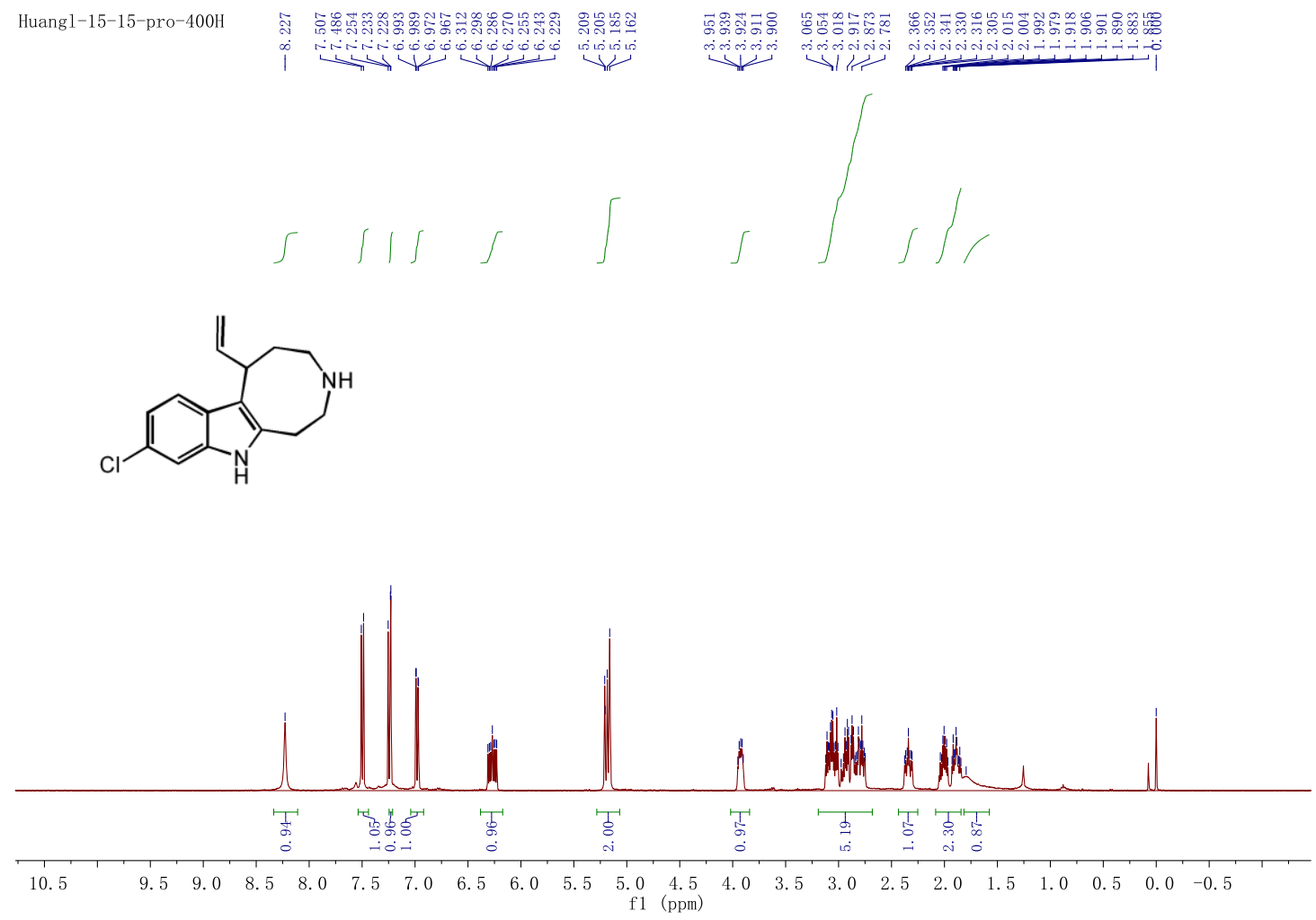
<smiles>C=CC1CCNCCc2[nH]c3cc(Cl)ccc3c21</smiles>
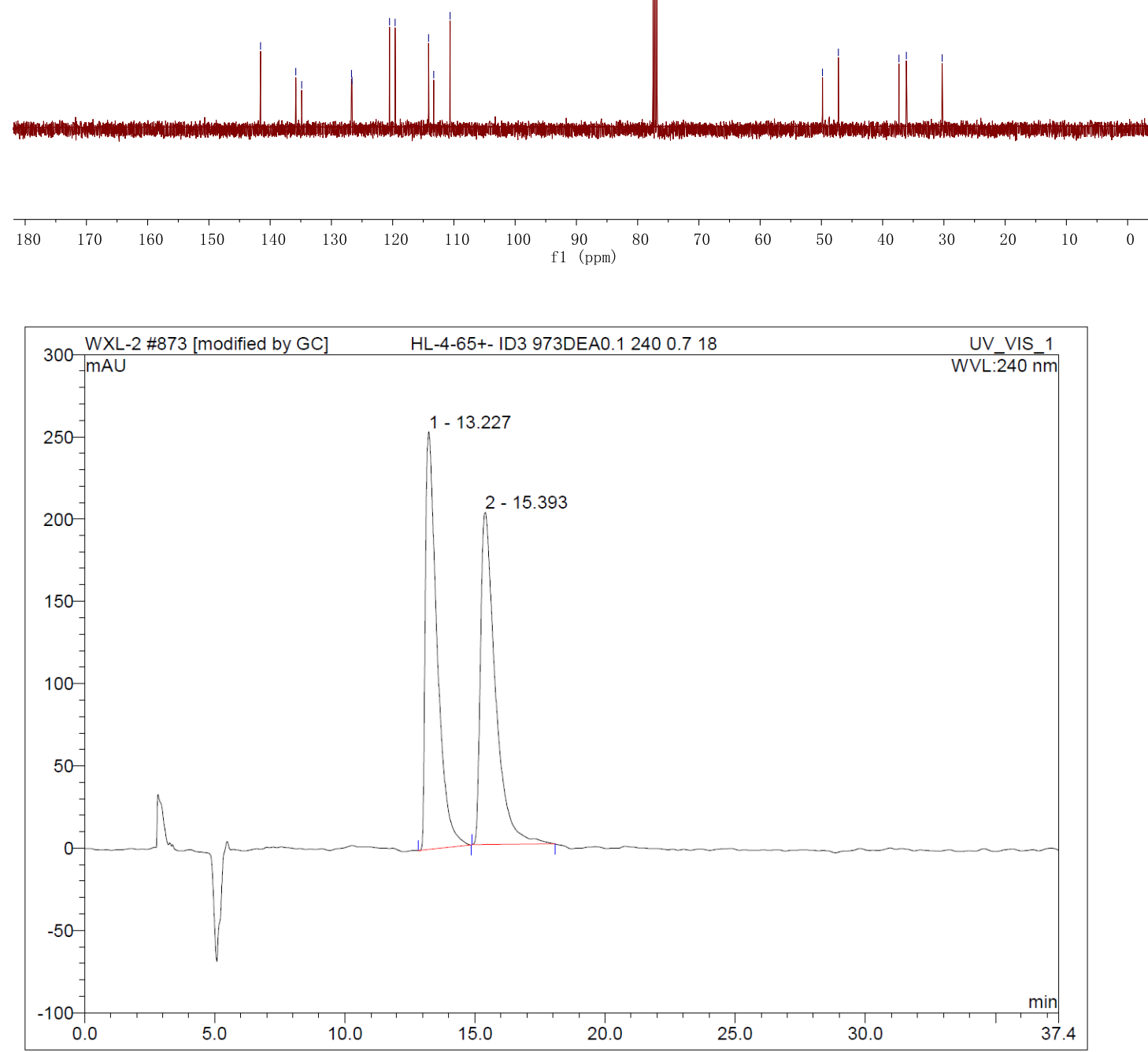

\begin{tabular}{|r|ccccccc|}
\hline No. & $\begin{array}{c}\text { Ret.Time } \\
\text { min }\end{array}$ & Peak Name & $\begin{array}{c}\text { Height } \\
\text { mAU }\end{array}$ & $\begin{array}{c}\text { Area } \\
\text { mAU*min }\end{array}$ & $\begin{array}{c}\text { Rel.Area } \\
\%\end{array}$ & Amount & Type \\
\hline 1 & 13.23 & n.a. & 253.719 & 132.239 & 49.72 & n.a. & BMB $^{*}$ \\
2 & 15.39 & n.a. & 201.888 & 133.702 & 50.28 & n.a. & BMB $^{*}$ \\
\hline Total: & & & 455.607 & 265.940 & 100.00 & 0.000 & \\
\hline
\end{tabular}




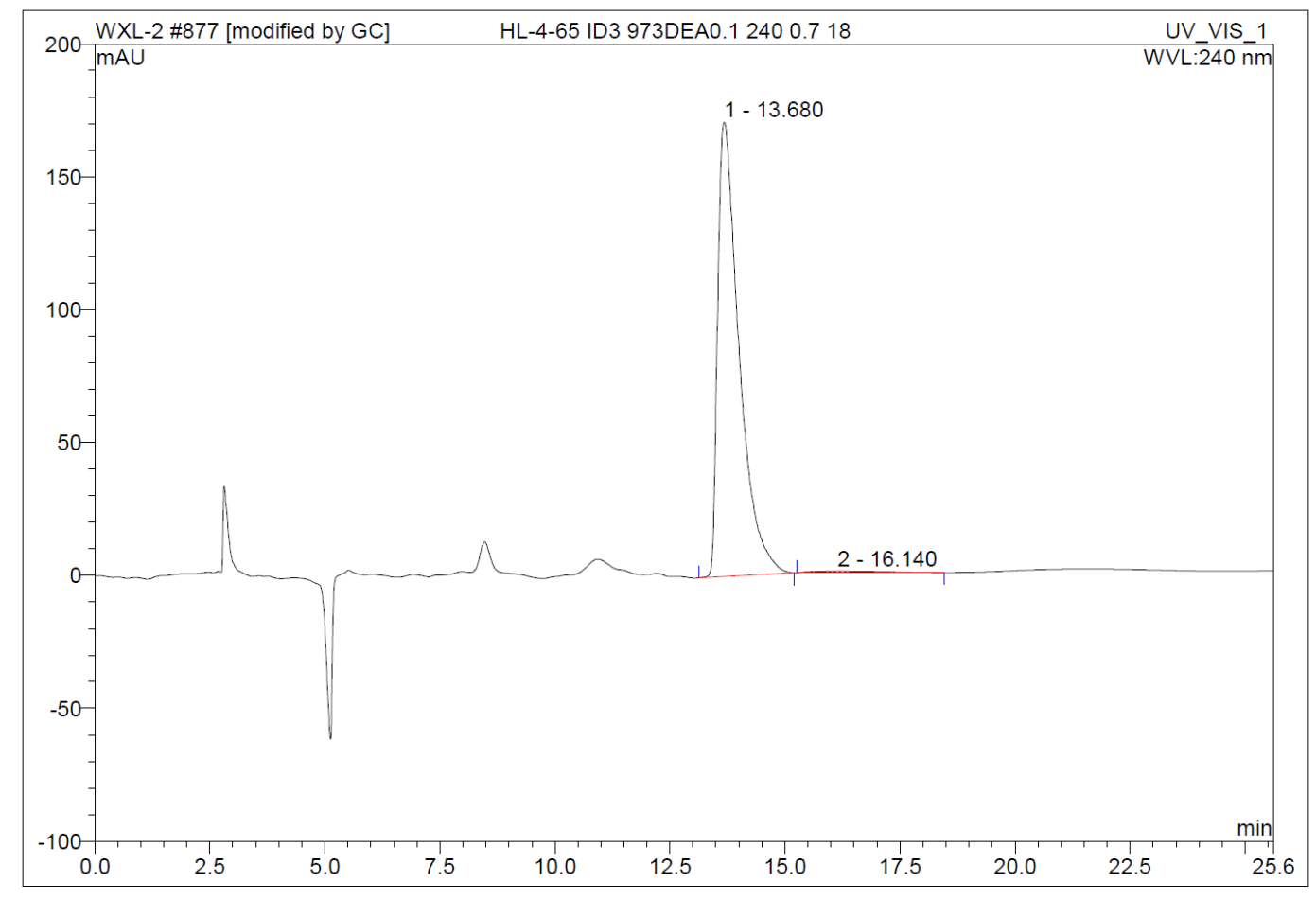

\begin{tabular}{|r|rrrrrrr|}
\hline No. & $\begin{array}{r}\text { Ret.Time } \\
\text { min }\end{array}$ & Peak Name & $\begin{array}{c}\text { Height } \\
\text { mAU }\end{array}$ & $\begin{array}{r}\text { Area } \\
\text { mAU*min }\end{array}$ & $\begin{array}{r}\text { Rel.Area } \\
\%\end{array}$ & Amount & Type \\
\hline 1 & 13.68 & n.a. & 170.929 & 92.179 & 98.81 & n.a. & BMB $^{*}$ \\
2 & 16.14 & n.a. & 0.606 & 1.111 & 1.19 & n.a. & BMB $^{*}$ \\
\hline Total: & & & 171.535 & 93.290 & 100.00 & 0.000 & \\
\hline
\end{tabular}

NMR Spectra and HPLC Chromatographs of 2n

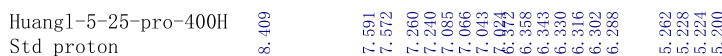

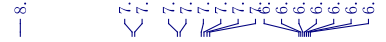

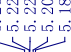

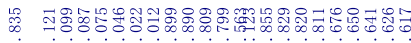

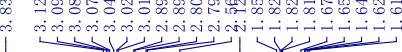
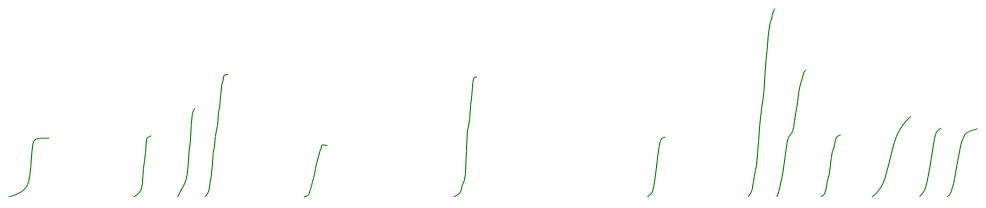<smiles>C=CC1CNCCCc2[nH]c3ccccc3c21</smiles>

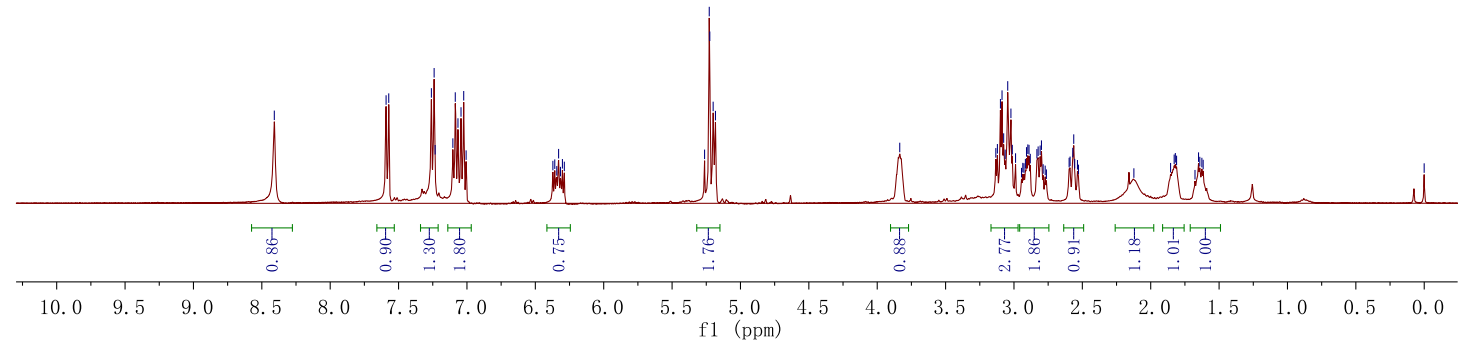


<smiles>C=CC1CNCCCc2[nH]c3ccccc3c21</smiles>

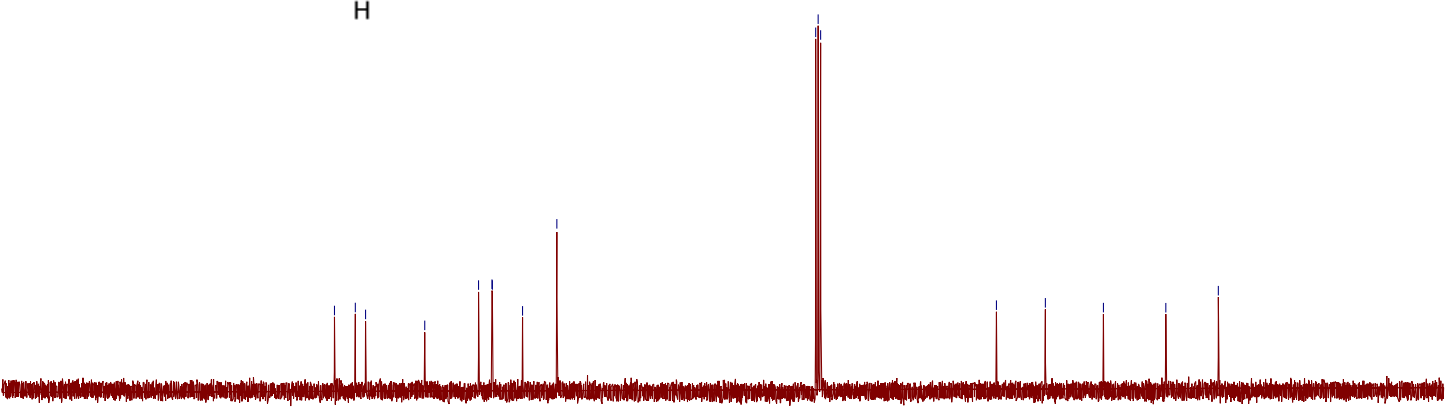

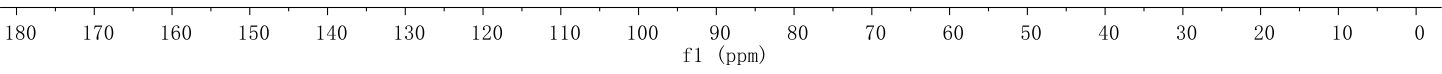

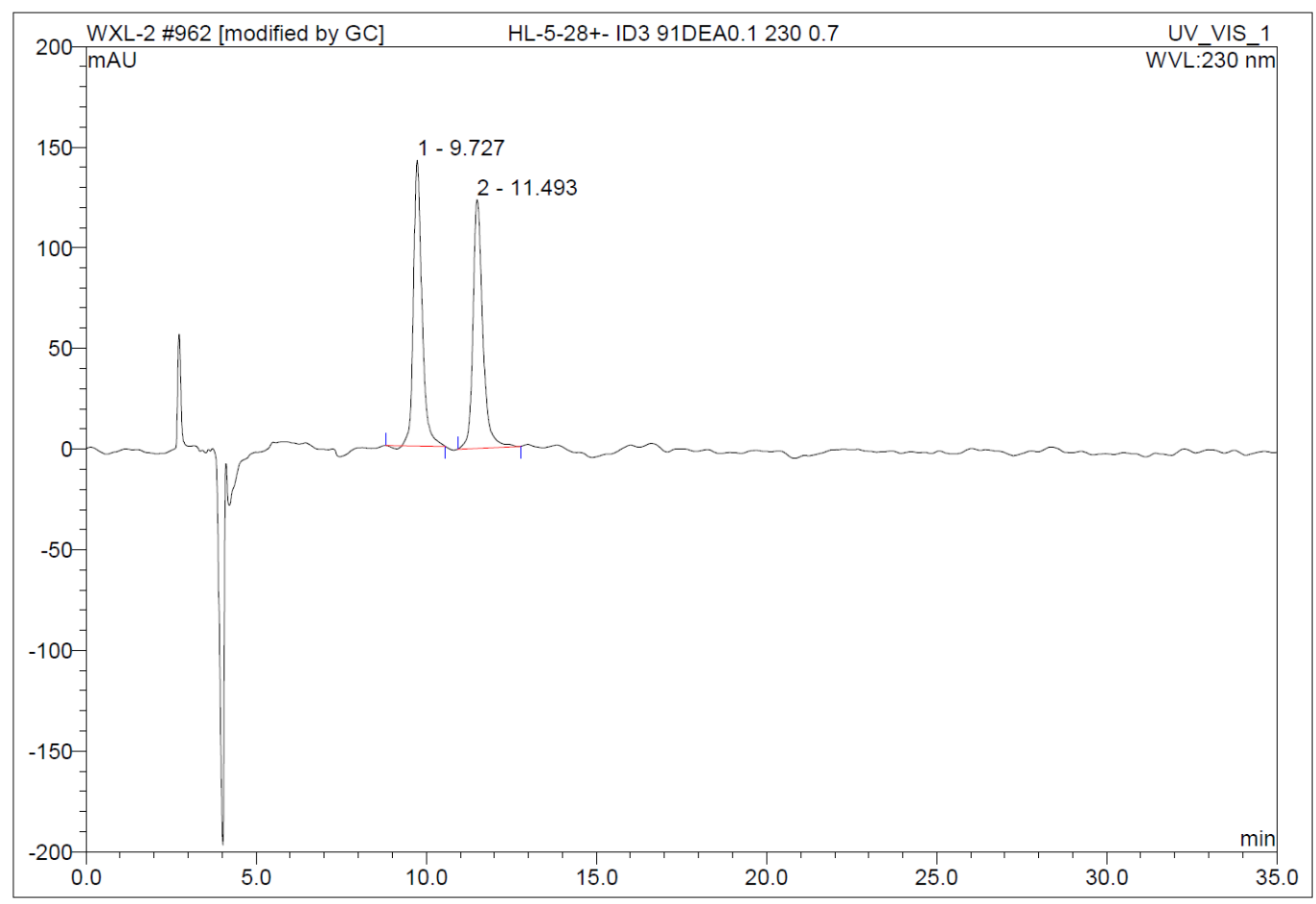

\begin{tabular}{|r|rrrrrrr|}
\hline No. & $\begin{array}{c}\text { Ret.Time } \\
\text { min }\end{array}$ & Peak Name & $\begin{array}{c}\text { Height } \\
\text { mAU }\end{array}$ & $\begin{array}{c}\text { Area } \\
\text { mAU*min }\end{array}$ & $\begin{array}{c}\text { Rel.Area } \\
\%\end{array}$ & Amount & Type \\
\hline 1 & 9.73 & n.a. & 141.920 & 41.045 & 49.85 & n.a. & BMB $^{*}$ \\
2 & 11.49 & n.a. & 123.511 & 41.288 & 50.15 & n.a. & BMB $^{*}$ \\
\hline Total: & & & 265.431 & 82.333 & 100.00 & 0.000 & \\
\hline
\end{tabular}




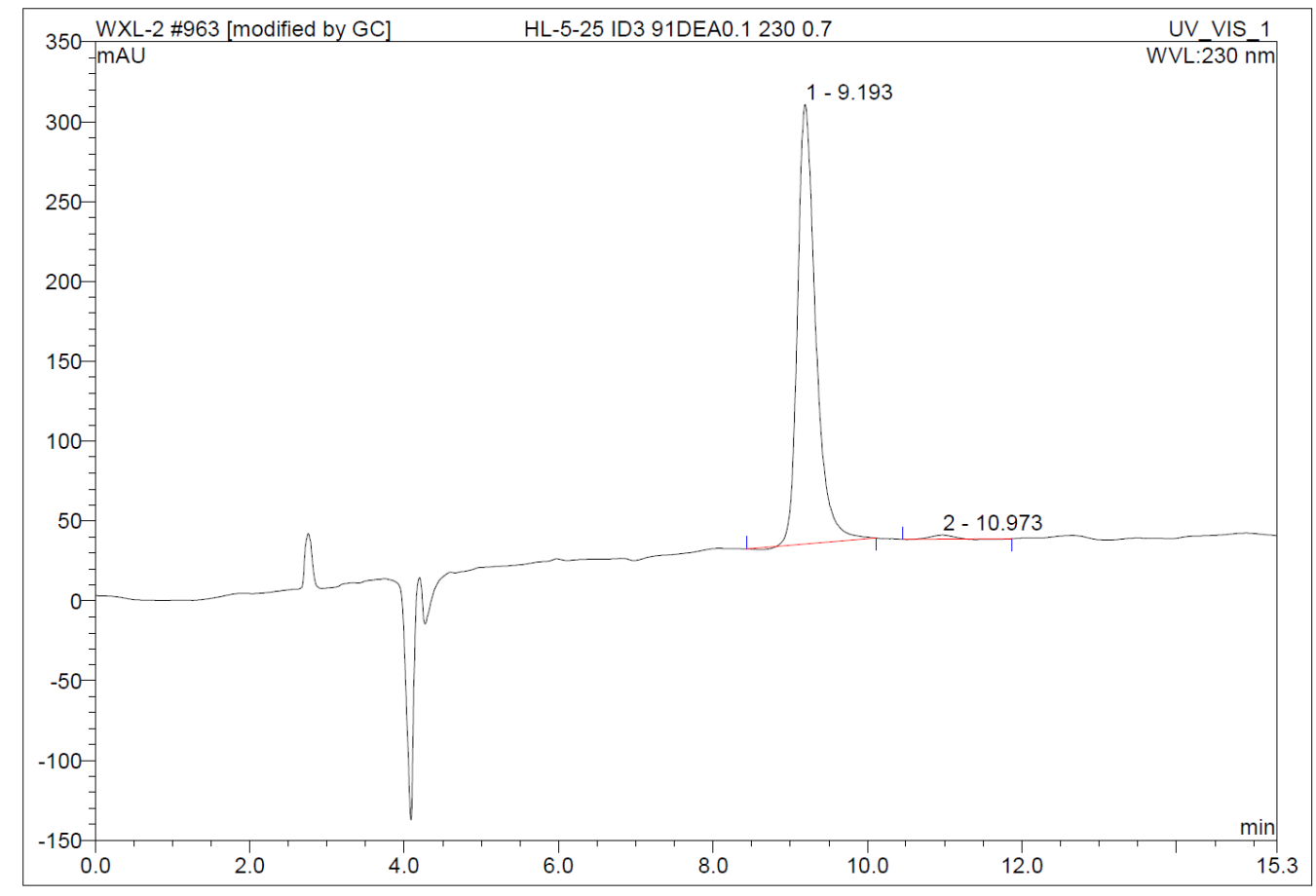

\begin{tabular}{|c|c|c|c|c|c|c|c|}
\hline No. & $\begin{array}{l}\text { Ret.Time } \\
\min \end{array}$ & Peak Name & $\begin{array}{c}\text { Height } \\
\text { mAU }\end{array}$ & $\begin{array}{c}\text { Area } \\
\text { mAU*min }\end{array}$ & $\begin{array}{c}\text { Rel.Area } \\
\%\end{array}$ & Amount & Type \\
\hline 1 & 9.19 & n.a. & 275.318 & 74.963 & 99.02 & n.a. & BMB $^{*}$ \\
\hline 2 & 10.97 & n.a. & 2.571 & 0.739 & 0.98 & n.a. & $\mathrm{BMB}^{*}$ \\
\hline Total: & & & 277.889 & 75.703 & 100.00 & 0.000 & \\
\hline
\end{tabular}

NMR Spectra and HPLC Chromatographs of 20

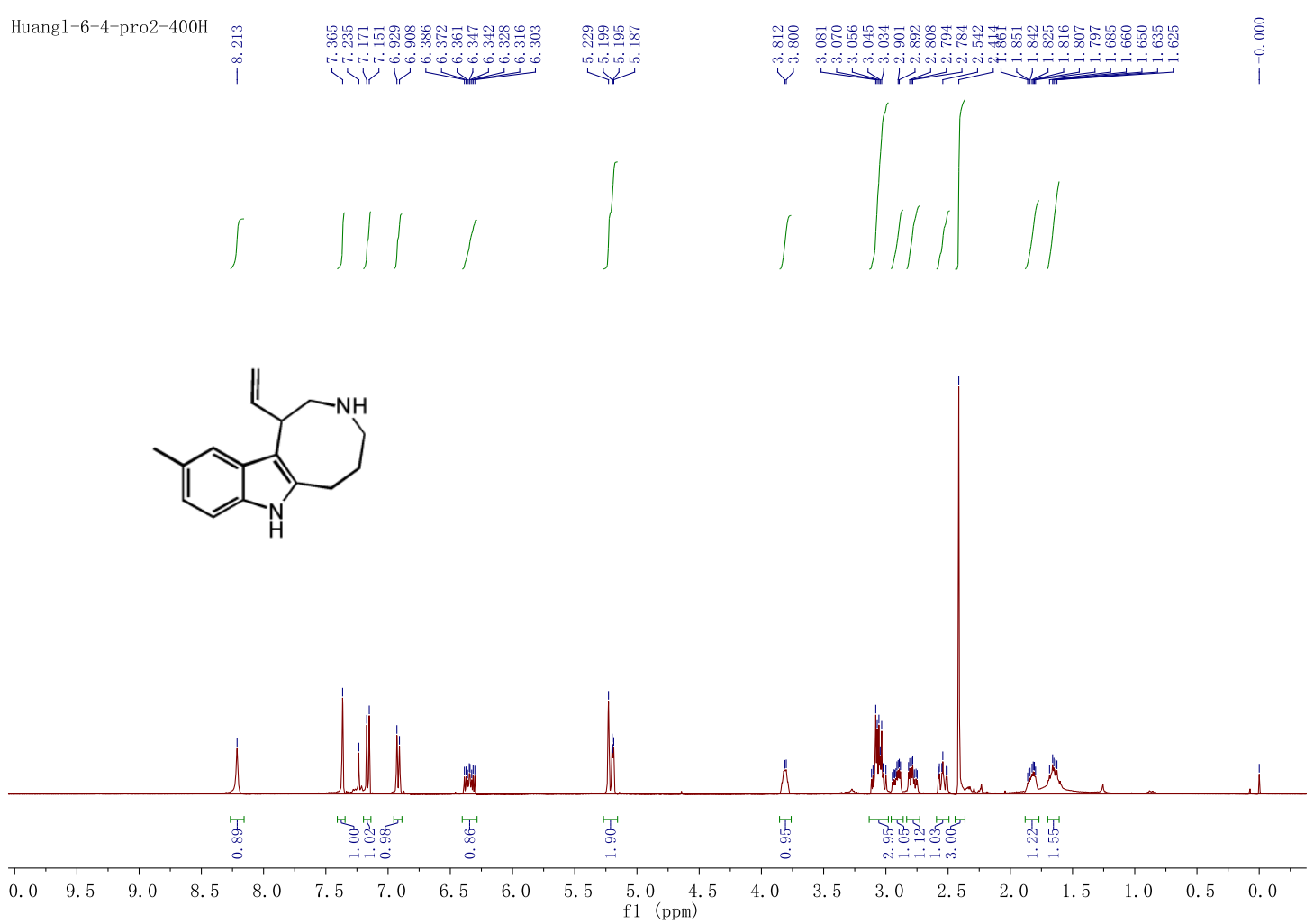


<smiles>C=CC1CNCCCc2[nH]c3ccc(C)cc3c21</smiles>
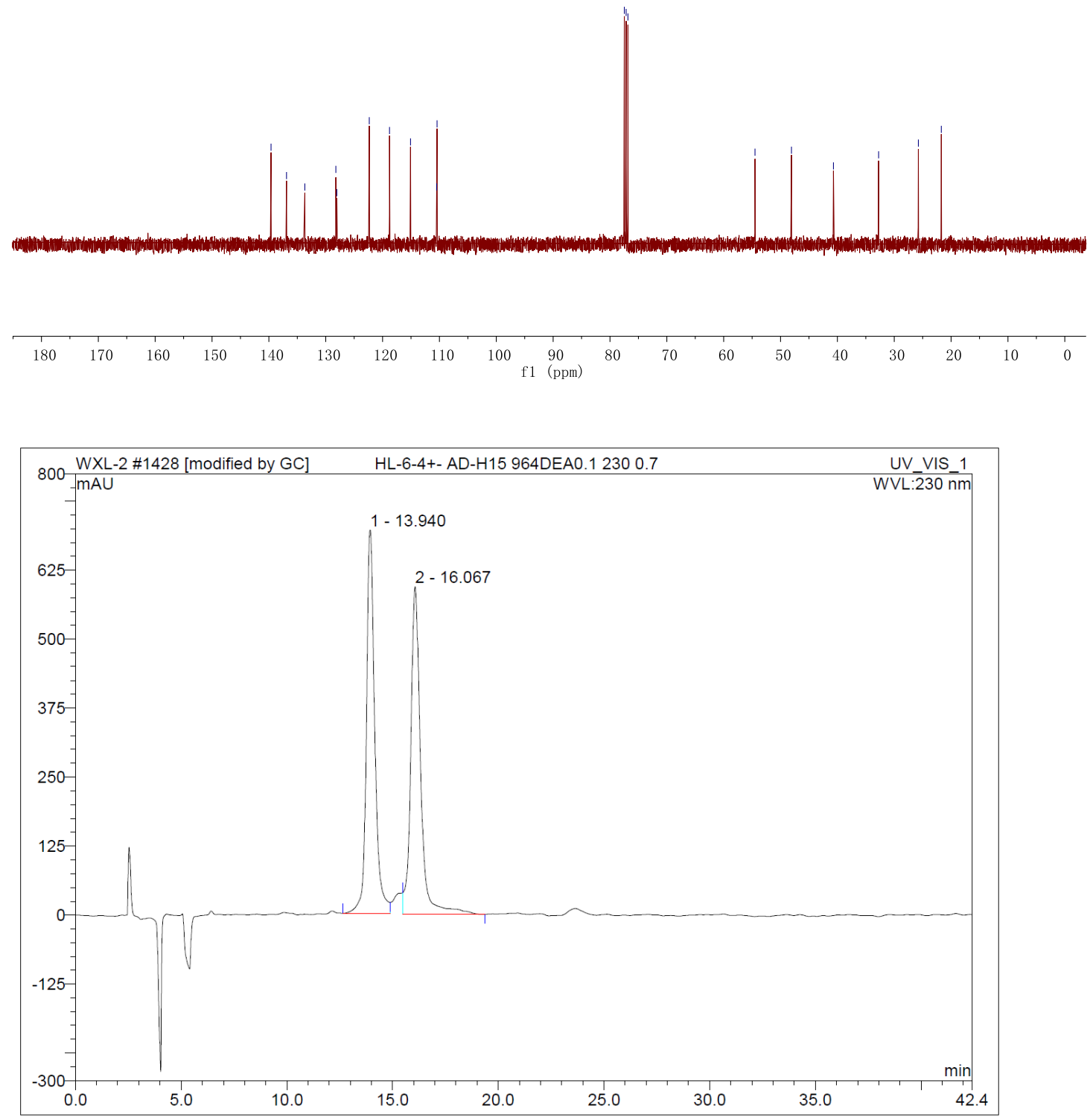

\begin{tabular}{|r|ccrrrrr|}
\hline No. & $\begin{array}{c}\text { Ret.Time } \\
\text { min }\end{array}$ & Peak Name & $\begin{array}{c}\text { Height } \\
\text { mAU }\end{array}$ & $\begin{array}{c}\text { Area } \\
\text { mAU*min }\end{array}$ & $\begin{array}{r}\text { Rel.Area } \\
\%\end{array}$ & Amount & Type \\
\hline 1 & 13.94 & n.a. & 694.382 & 318.538 & 49.80 & n.a. & BM $^{*}$ \\
2 & 16.07 & n.a. & 593.637 & 321.128 & 50.20 & n.a. & MB $^{*}$ \\
\hline Total: & & & 1288.019 & 639.666 & 100.00 & 0.000 & \\
\hline
\end{tabular}




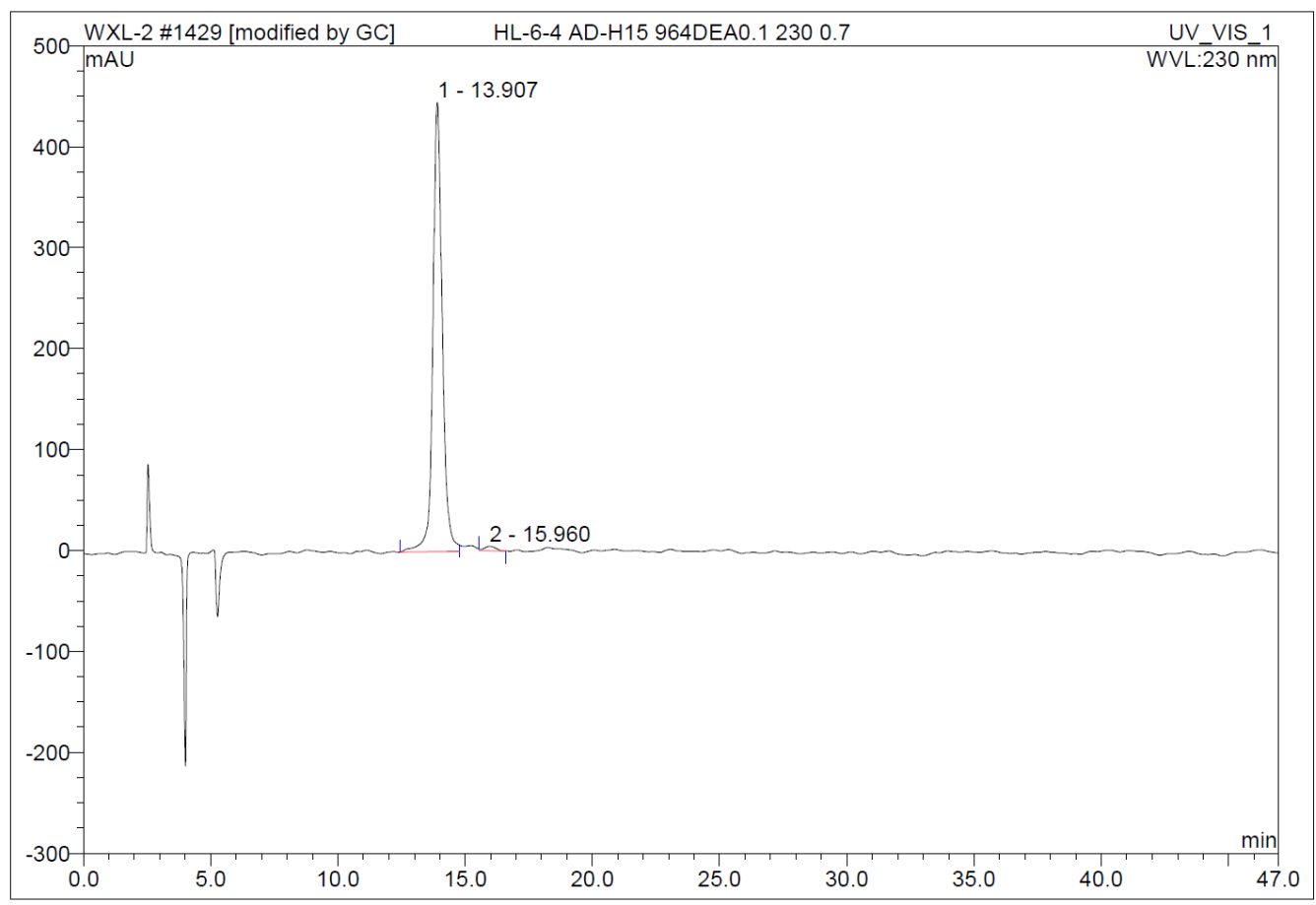

\begin{tabular}{|r|ccrrrrr|}
\hline No. & $\begin{array}{c}\text { Ret.Time } \\
\text { min }\end{array}$ & Peak Name & $\begin{array}{c}\text { Height } \\
\text { mAU }\end{array}$ & $\begin{array}{c}\text { Area } \\
\text { mAU*min }\end{array}$ & $\begin{array}{r}\text { Rel.Area } \\
\%\end{array}$ & Amount & Type \\
\hline 1 & 13.91 & n.a. & 444.742 & 190.832 & 98.79 & n.a. & BM $^{*}$ \\
2 & 15.96 & n.a. & 4.491 & 2.332 & 1.21 & n.a. & MB $^{*}$ \\
\hline Total: & & & 449.233 & 193.164 & 100.00 & 0.000 & \\
\hline
\end{tabular}

NMR Spectra and HPLC Chromatographs of $\mathbf{2 p}$

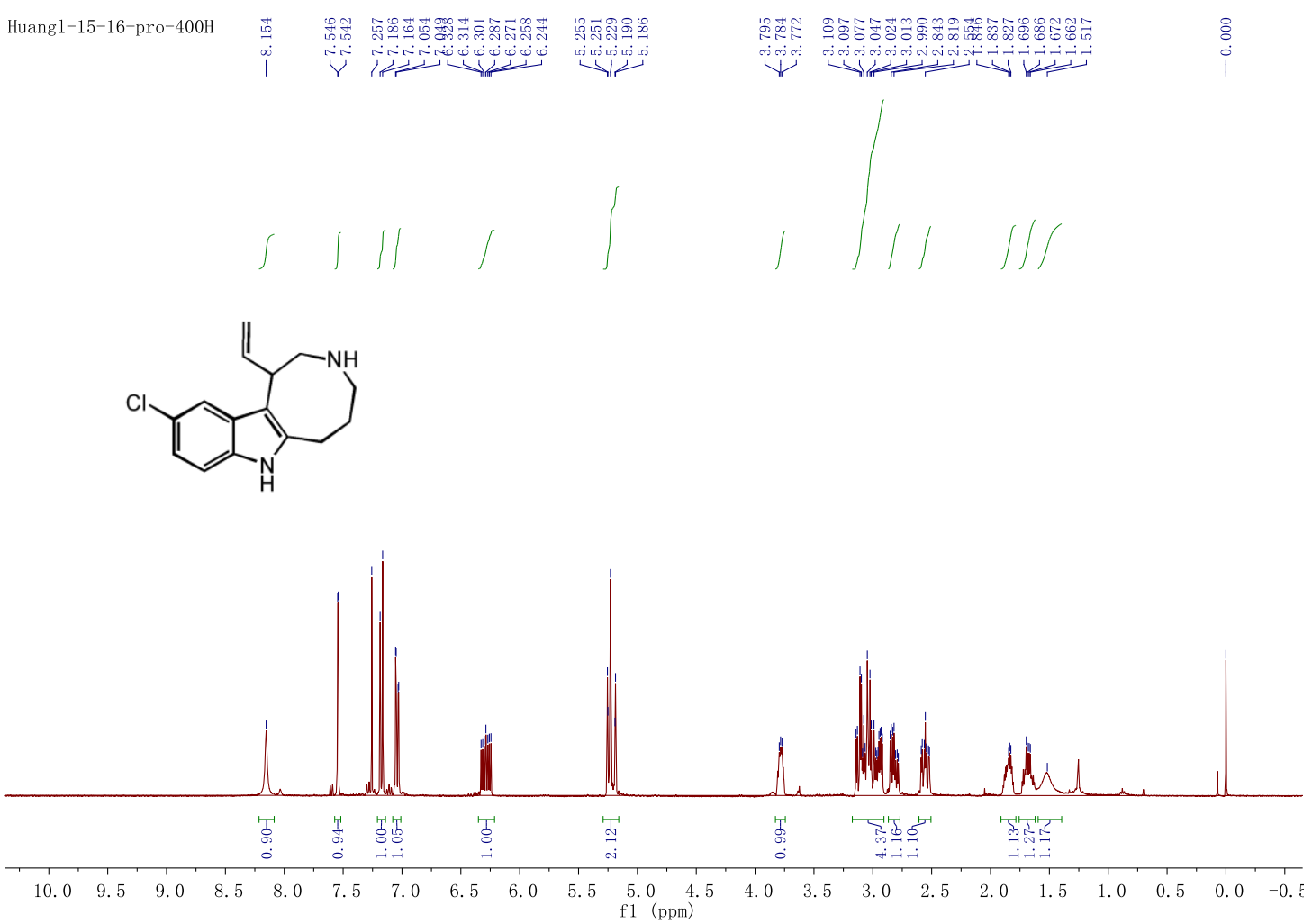


<smiles>C=CC1CNCCCc2[nH]c3ccc(Cl)cc3c21</smiles>

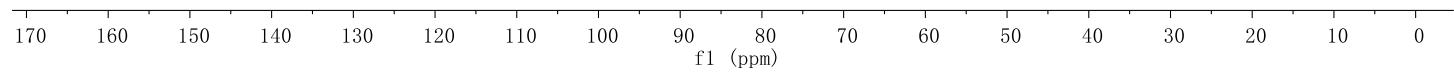

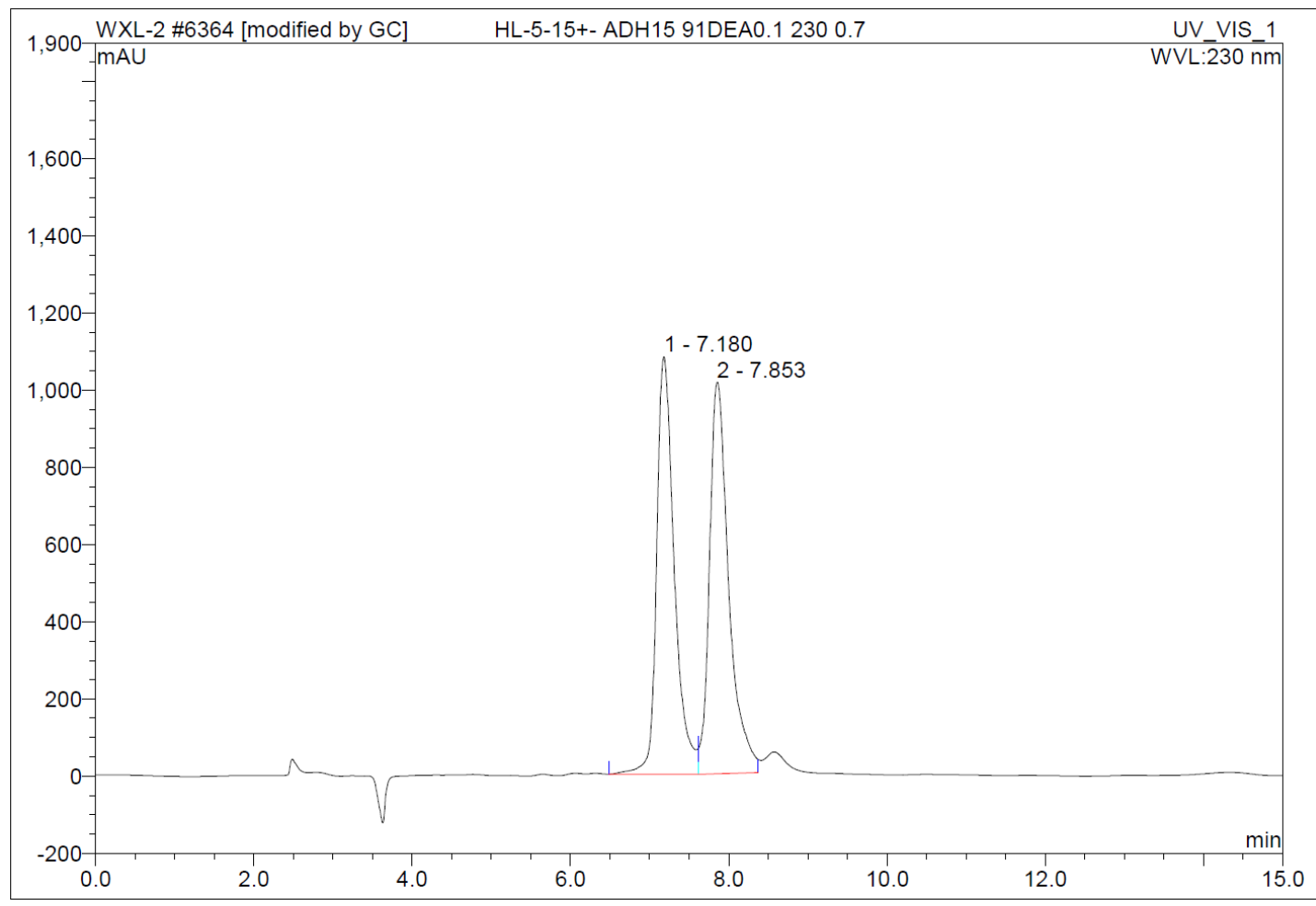

\begin{tabular}{|r|cccrrrr|}
\hline No. & $\begin{array}{c}\text { Ret.Time } \\
\text { min }\end{array}$ & Peak Name & $\begin{array}{c}\text { Height } \\
\text { mAU }\end{array}$ & $\begin{array}{c}\text { Area } \\
\text { mAU*min }\end{array}$ & $\begin{array}{c}\text { Rel.Area } \\
\%\end{array}$ & Amount & Type \\
\hline 1 & 7.18 & n.a. & 1082.155 & 280.771 & 49.49 & n.a. & BM $^{*}$ \\
2 & 7.85 & n.a. & 1014.272 & 286.575 & 50.51 & n.a. & M $^{*}$ \\
\hline Total: & & & 2096.428 & 567.346 & 100.00 & 0.000 & \\
\hline
\end{tabular}




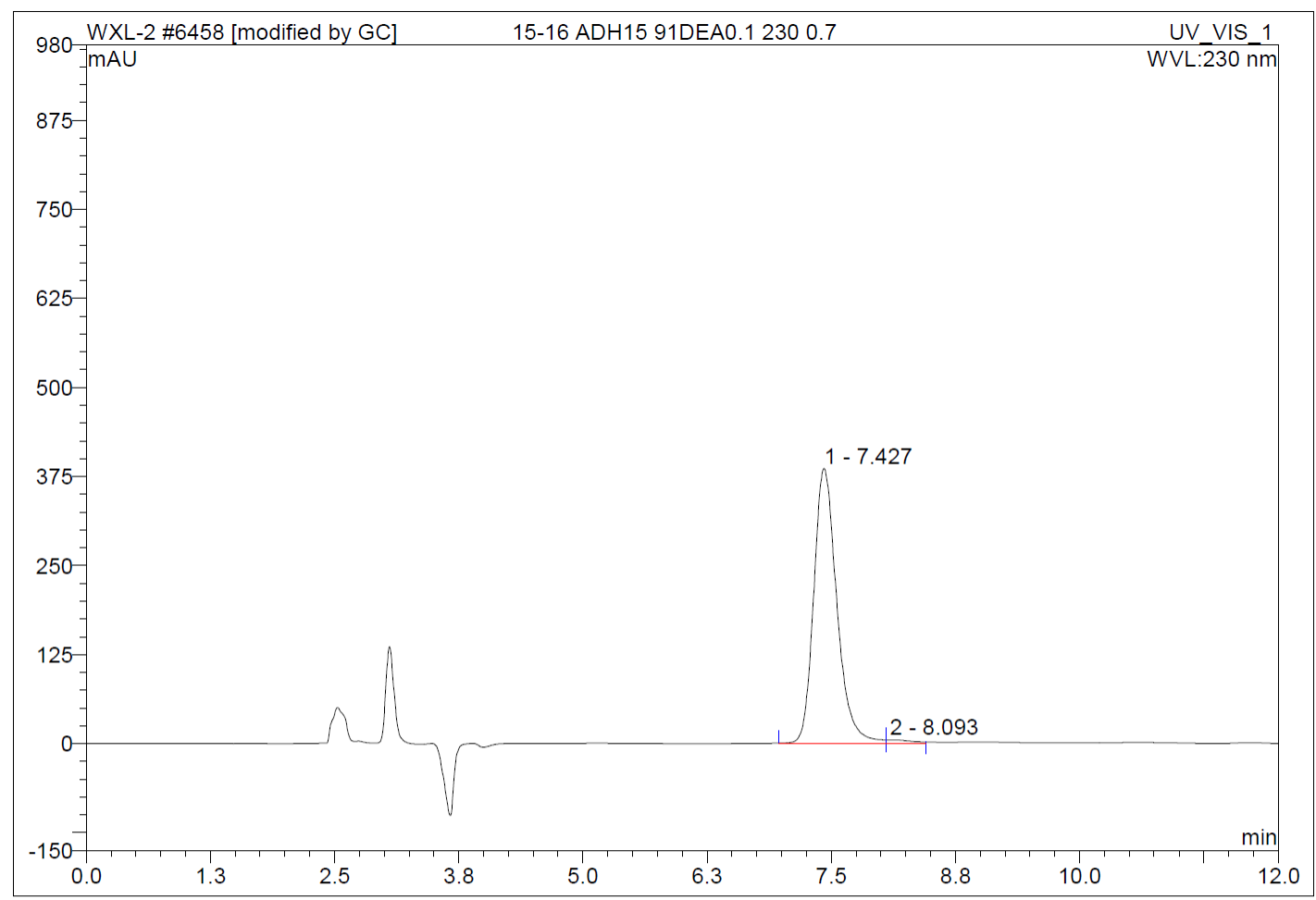

\begin{tabular}{|r|rrrrrrr|}
\hline No. & $\begin{array}{c}\text { Ret.Time } \\
\text { min }\end{array}$ & Peak Name & $\begin{array}{c}\text { Height } \\
\text { mAU }\end{array}$ & $\begin{array}{c}\text { Area } \\
\text { mAU*min }\end{array}$ & $\begin{array}{r}\text { Rel.Area } \\
\%\end{array}$ & Amount & Type \\
\hline 1 & 7.43 & n.a. & 385.729 & 103.981 & 98.84 & n.a. & BM $^{*}$ \\
2 & 8.09 & n.a. & 4.468 & 1.222 & 1.16 & n.a. & $M^{*}$ \\
\hline Total: & & & 390.197 & 105.202 & 100.00 & 0.000 & \\
\hline
\end{tabular}

NMR Spectra and HPLC Chromatographs of $\mathbf{2 q}$

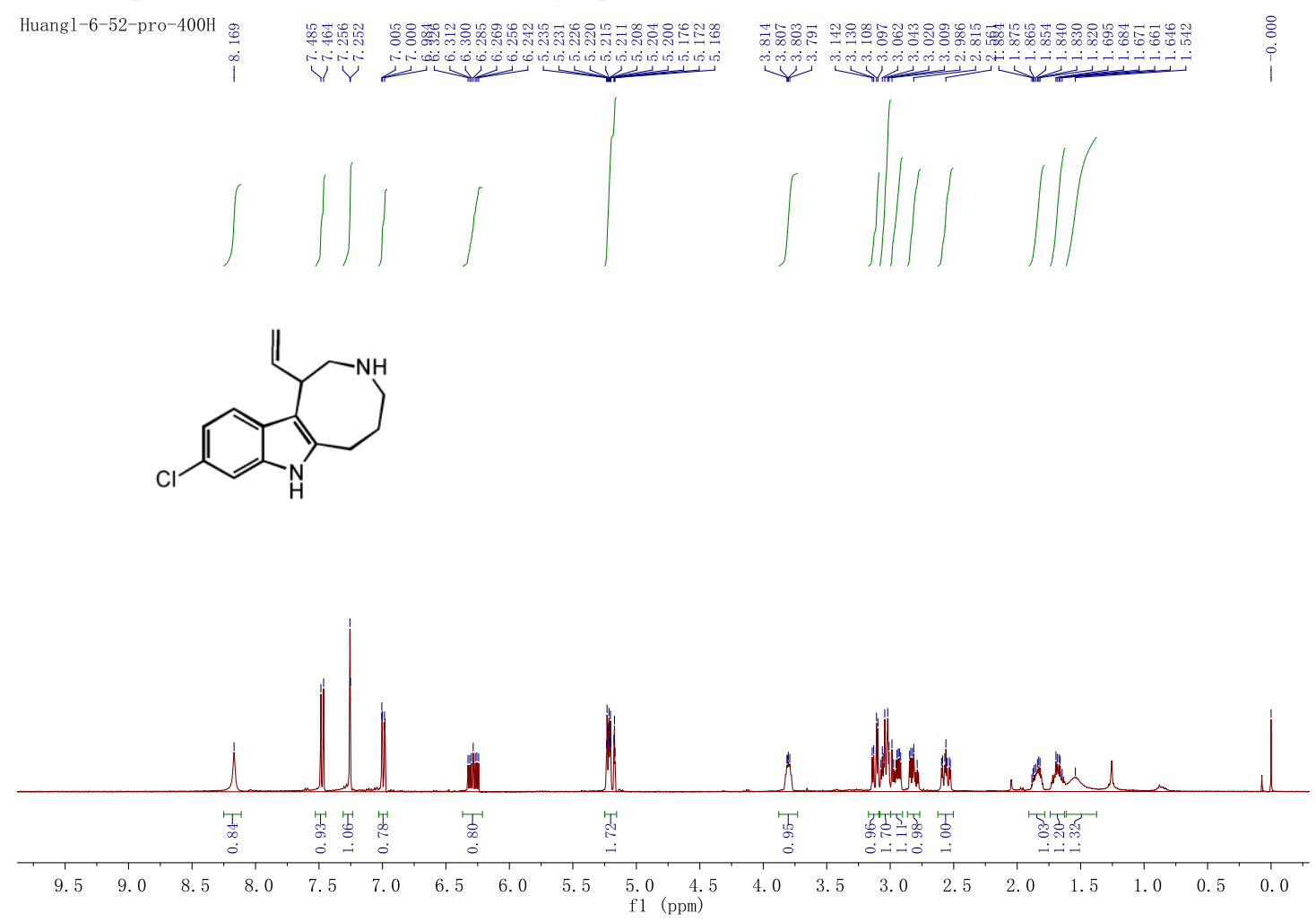


<smiles>C=CC1CNCCCc2[nH]c3cc(Cl)ccc3c21</smiles>
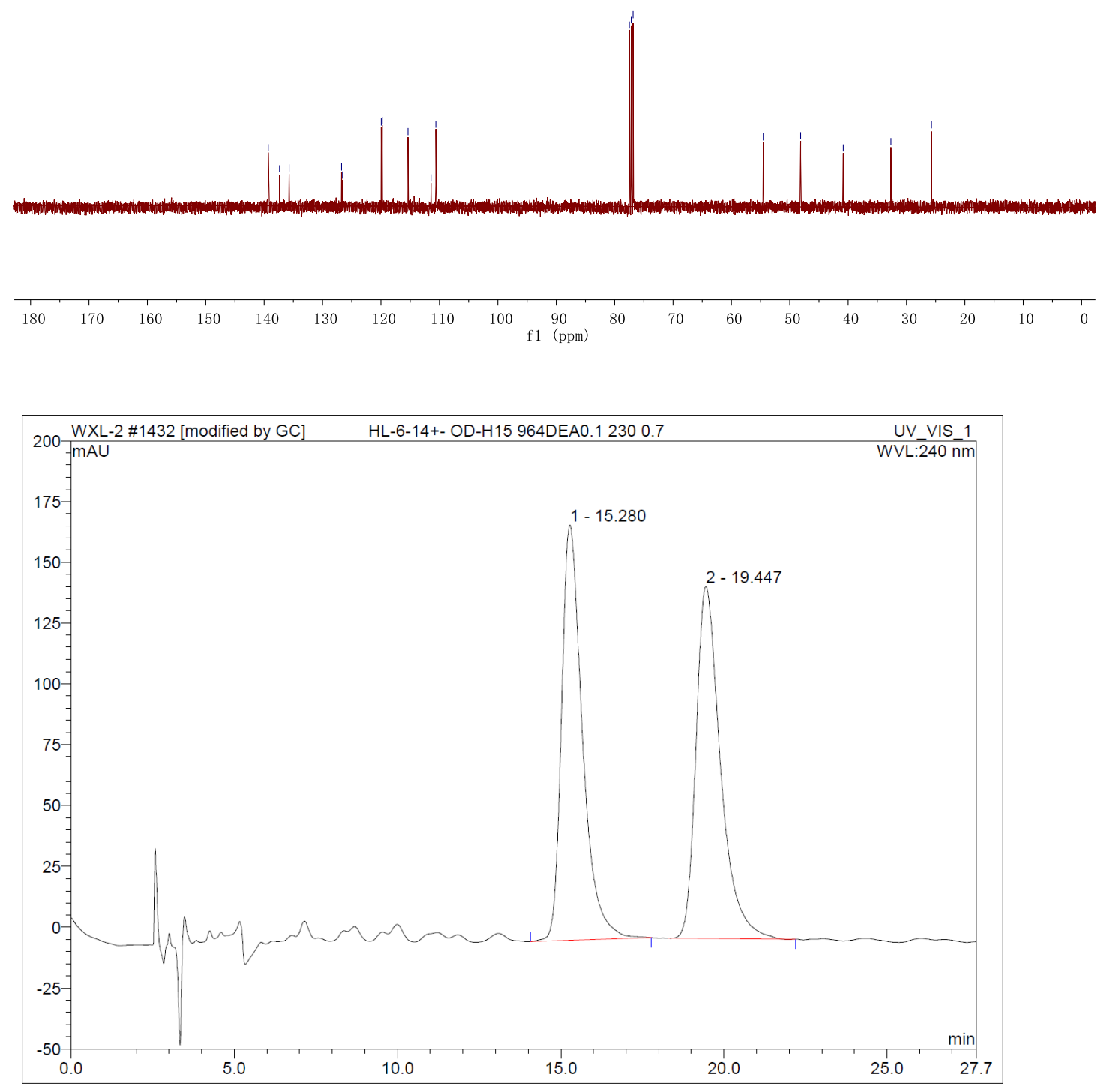

\begin{tabular}{|r|ccccccc|}
\hline No. & $\begin{array}{c}\text { Ret.Time } \\
\text { min }\end{array}$ & Peak Name & $\begin{array}{c}\text { Height } \\
\text { mAU }\end{array}$ & $\begin{array}{c}\text { Area } \\
\text { mAU*min }\end{array}$ & $\begin{array}{c}\text { Rel.Area } \\
\%\end{array}$ & Amount & Type \\
\hline 1 & 15.28 & n.a. & 170.729 & 126.842 & 50.17 & n.a. & BMB $^{*}$ \\
2 & 19.45 & n.a. & 144.613 & 125.974 & 49.83 & n.a. & BMB $^{*}$ \\
\hline Total: & & & 315.342 & 252.816 & 100.00 & 0.000 & \\
\hline
\end{tabular}




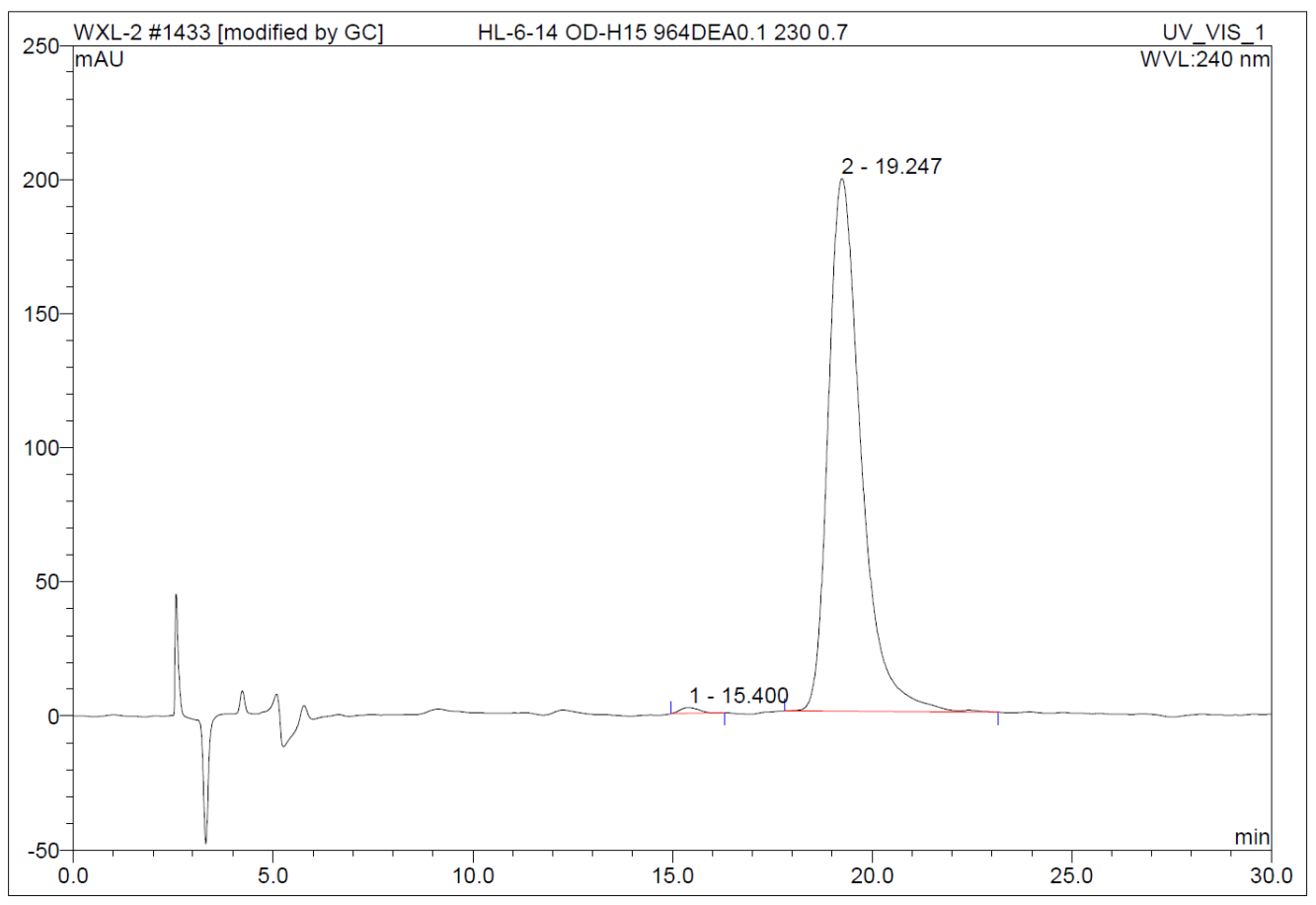

\begin{tabular}{|r|ccrrrrr|}
\hline No. & $\begin{array}{c}\text { Ret.Time } \\
\text { min }\end{array}$ & Peak Name & $\begin{array}{c}\text { Height } \\
\text { mAU }\end{array}$ & $\begin{array}{r}\text { Area } \\
\text { mAU*min }\end{array}$ & $\begin{array}{r}\text { Rel.Area } \\
\%\end{array}$ & Amount & Type \\
\hline 1 & 15.40 & n.a. & 2.174 & 1.163 & 0.61 & n.a. & BMB $^{*}$ \\
2 & 19.25 & n.a. & 198.724 & 189.178 & 99.39 & n.a. & BMB $^{*}$ \\
\hline Total: & & & 200.898 & 190.341 & 100.00 & 0.000 & \\
\hline
\end{tabular}

NMR Spectra and HPLC Chromatographs of 2r

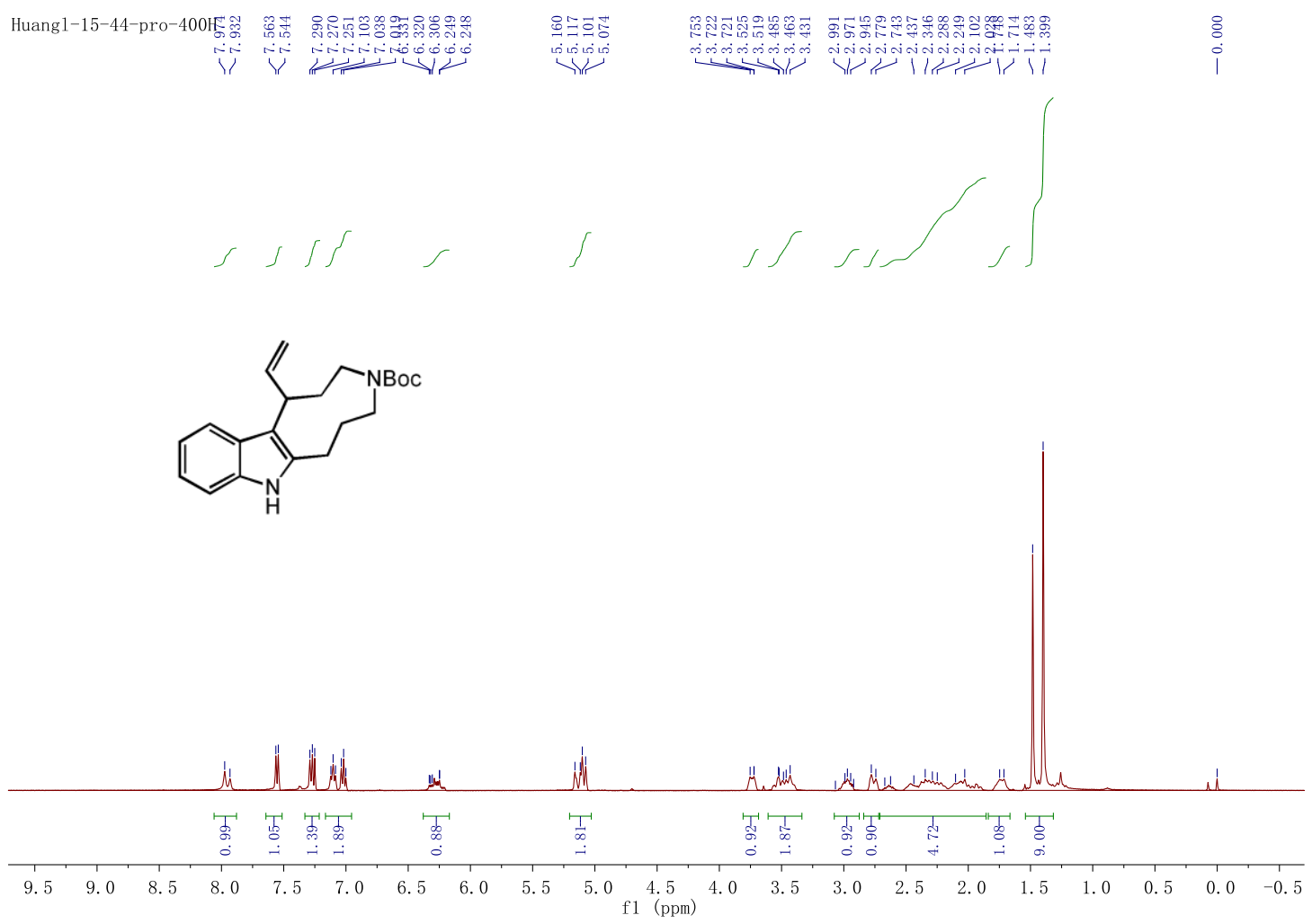


<smiles>C=CC(CCCNC(=O)OCc1ccccc1)c1c(CCCNC(C)(C)C)[nH]c2ccccc12</smiles>

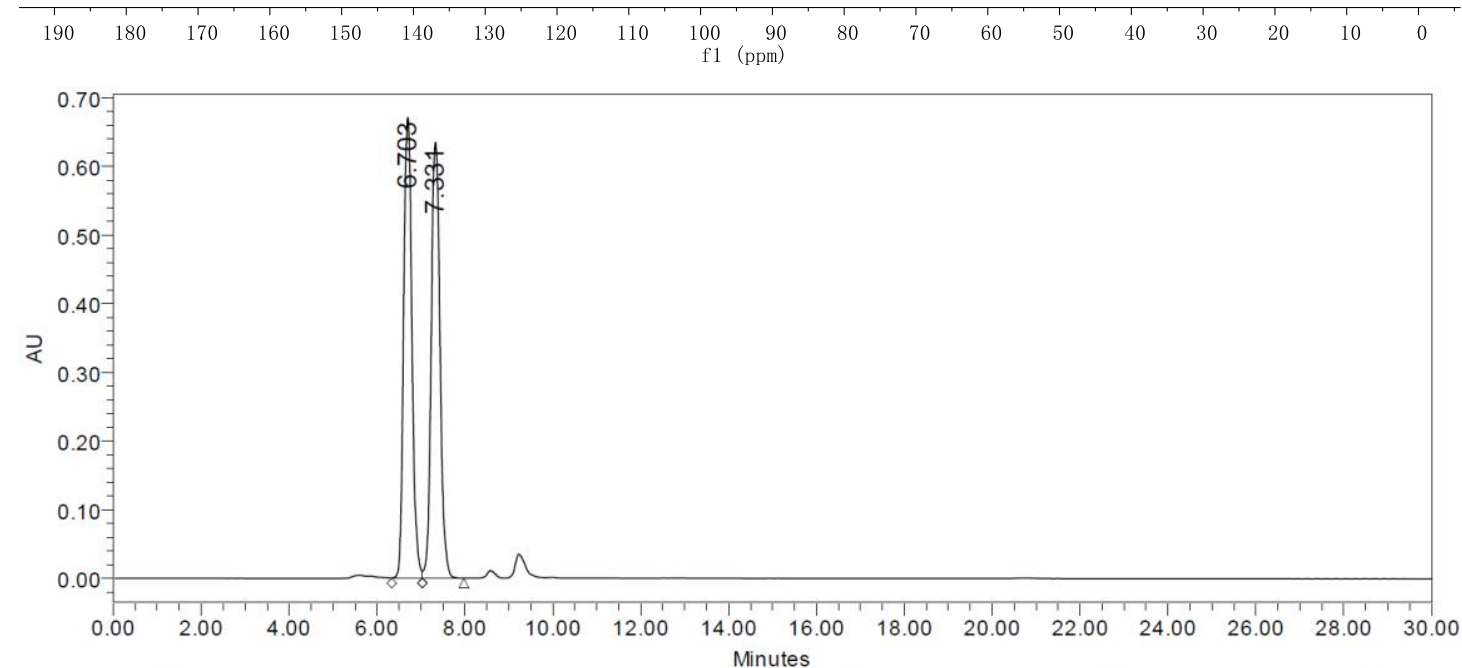

Channel: W2489 ChA; Processed Channel: W2489 ChA 230nm; Injection: 1; Date Acquired: 1/23/2016 11:10:38 AM CST; Result Id: 3065; Processing Method: 456445

\section{Peak Name:}

\begin{tabular}{|c|r|c|c|r|c|}
\hline & Injection & RT & Area & \% Area & Height \\
\hline 1 & 1 & 7.331 & 8796437 & 49.97 & 634985 \\
\hline 2 & 1 & 6.703 & 8805803 & 50.03 & 671691 \\
\hline Mean & & 7.017 & & & \\
\hline Std. Dev. & & 0.444 & & & \\
\hline \% RSD & & 6.32 & & & \\
\hline
\end{tabular}




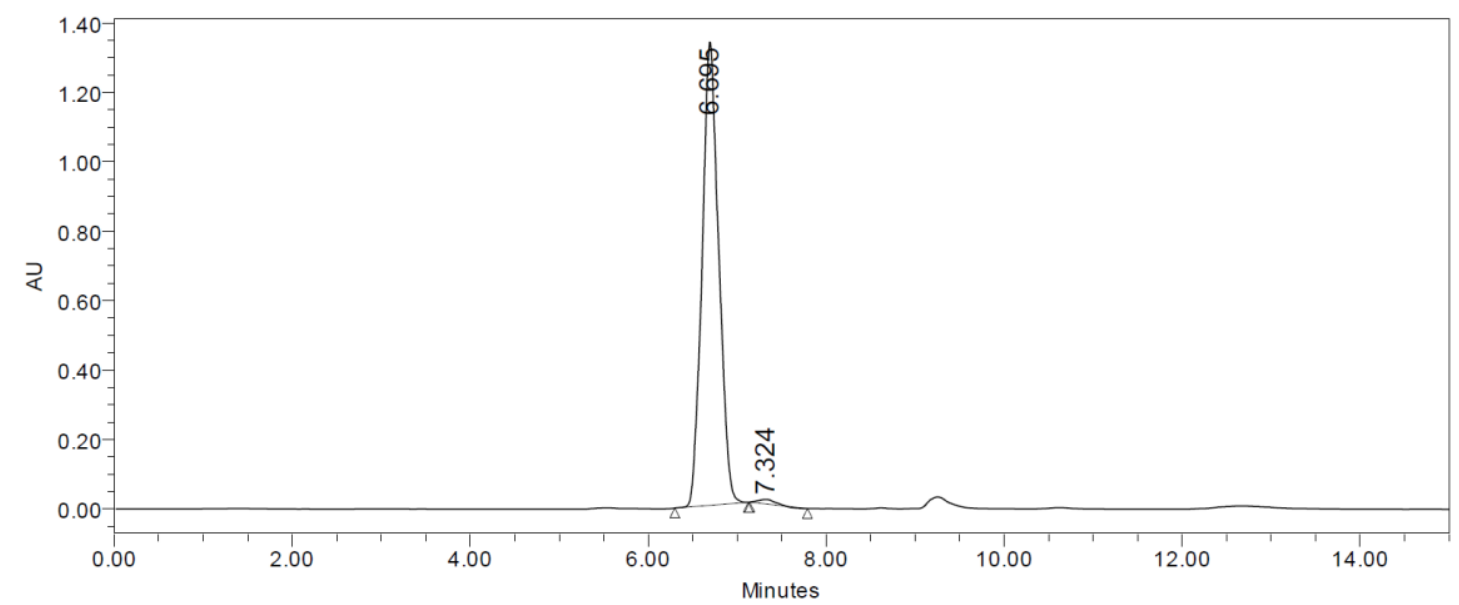

Channel: W2489 ChA; Processed Channel: W2489 ChA 230nm; Injection: 1; Date Acquired: 1/23/2016 1:07:12 PM CST; Result Id: 3067; Processing Method: bhjfg

Peak Name:

\begin{tabular}{|c|r|c|c|r|c|}
\hline & Injection & RT & Area & \% Area & Height \\
\hline 1 & 1 & 7.324 & 188344 & 1.08 & 13055 \\
\hline 2 & 1 & 6.695 & 17330020 & 98.92 & 1334968 \\
\hline Mean & & 7.009 & & & \\
\hline Std. Dev. & & 0.445 & & & \\
\hline$\%$ RSD & & 6.35 & & & \\
\hline
\end{tabular}

NMR Spectra and HPLC Chromatographs of $2 \mathrm{~s}$

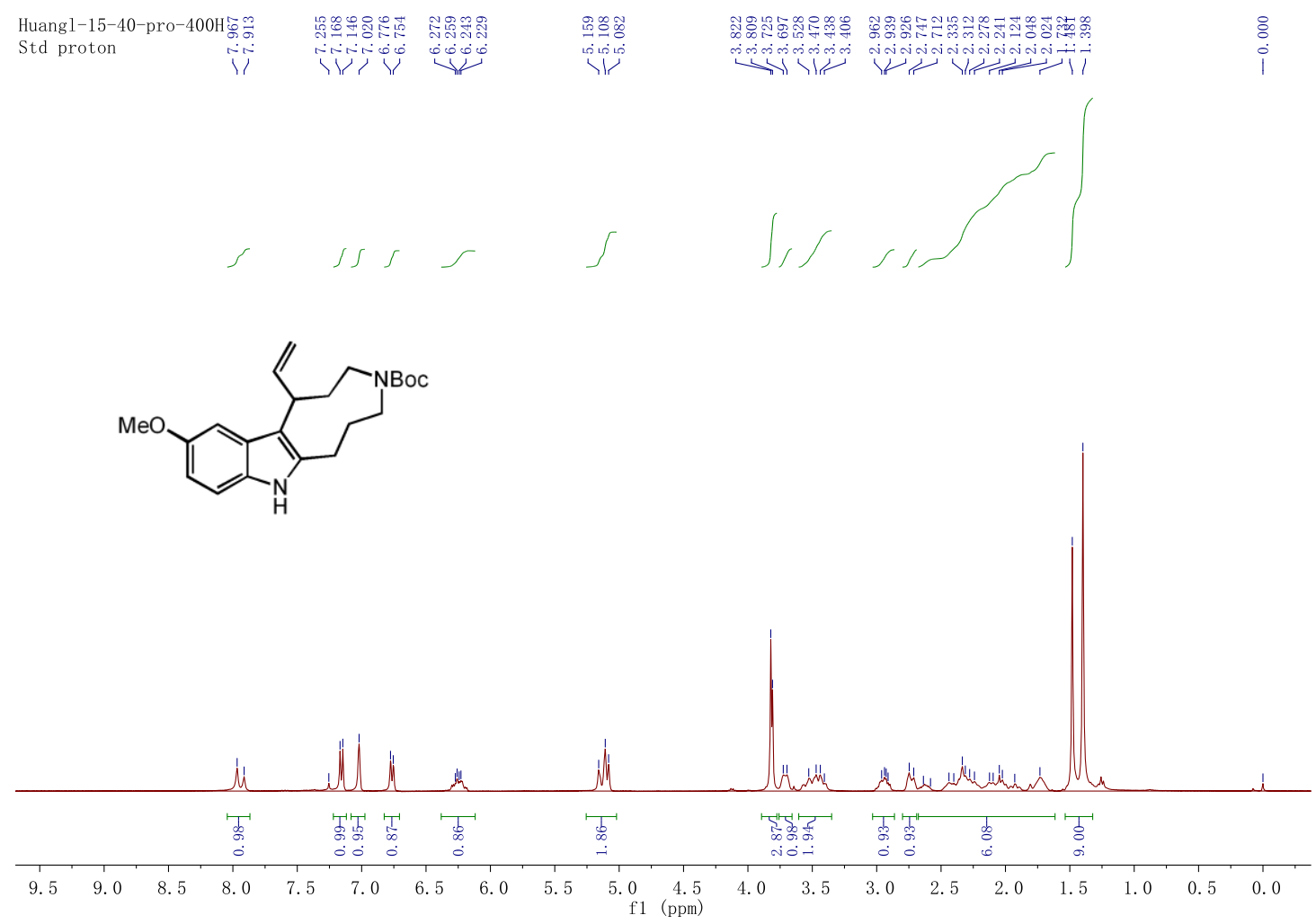


<smiles></smiles>
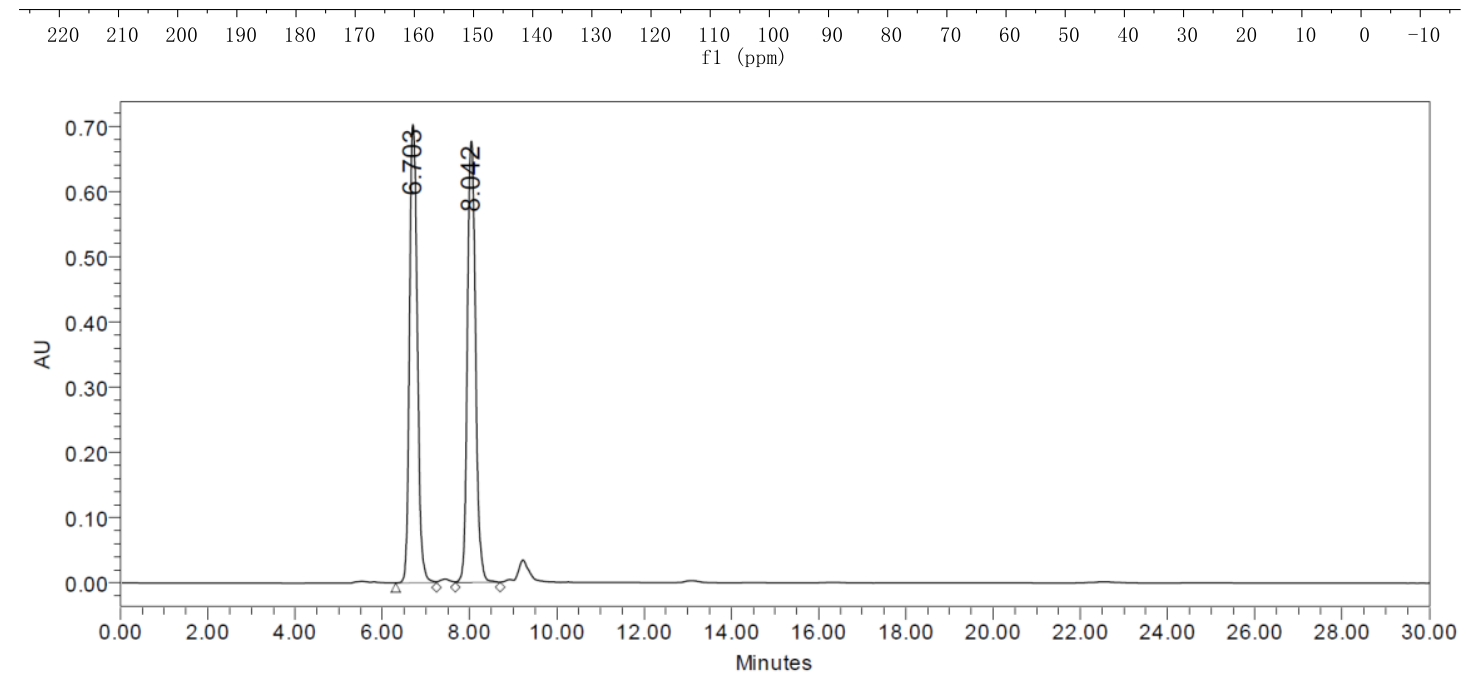

Channel: W2489 ChA; Processed Channel: W2489 ChA 230nm; Injection: 1; Date Acquired: 1/23/2016 10:40:13 AM CST; Result Id: 3061; Processing Method: 5424

Peak Name:

\begin{tabular}{|c|r|c|c|r|c|}
\hline & Injection & RT & Area & $\%$ Area & Height \\
\hline 1 & 1 & 8.042 & 8818906 & 50.14 & 676401 \\
\hline 2 & 1 & 6.703 & 8770875 & 49.86 & 702717 \\
\hline Mean & & 7.372 & & & \\
\hline Std. Dev. & & 0.946 & & & \\
\hline \% RSD & & 12.84 & & & \\
\hline
\end{tabular}




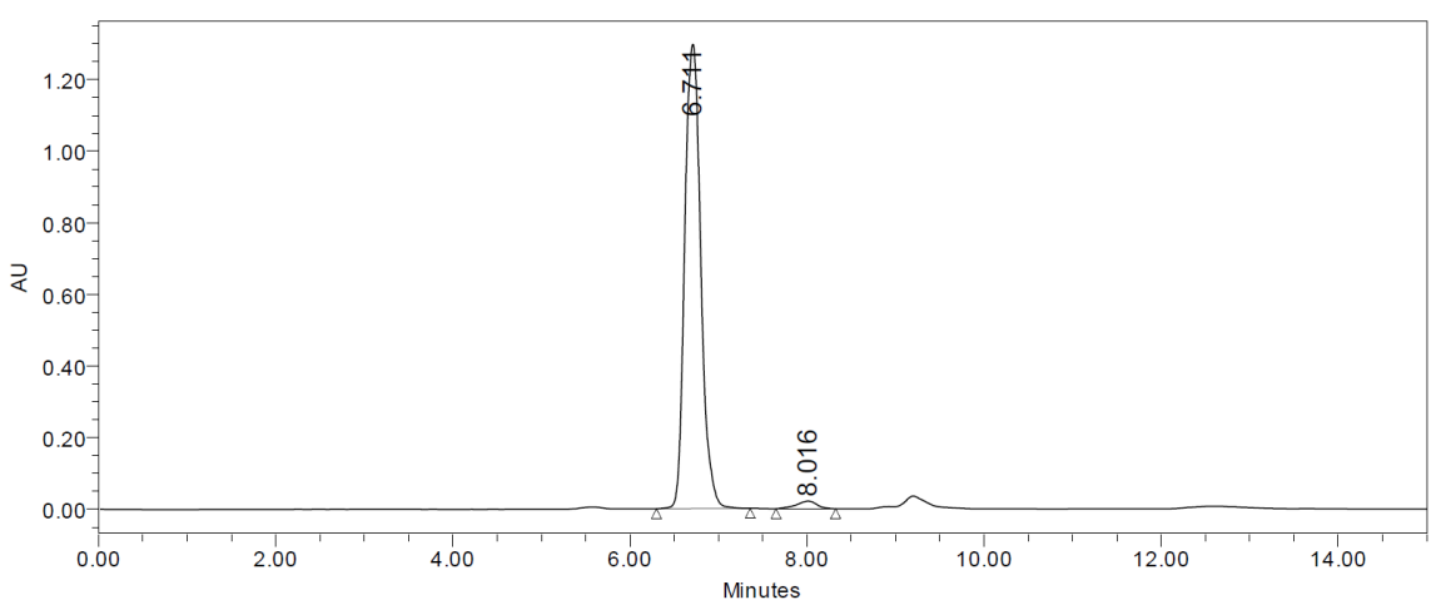

Channel: W2489 ChA; Processed Channel: W2489 ChA 230nm; Injection: 1; Date Acquired: 1/23/2016 1:55:24 PM CST; Result Id: 3063; Processing Method: 4564

Peak Name:

\begin{tabular}{|c|r|c|c|r|r|}
\hline & Injection & RT & Area & \% Area & Height \\
\hline 1 & 1 & 8.016 & 327357 & 1.96 & 20493 \\
\hline 2 & 1 & 6.711 & 16334406 & 98.04 & 1296656 \\
\hline Mean & & 7.364 & & & \\
\hline Std. Dev. & & 0.922 & & & \\
\hline \% RSD & & 12.53 & & & \\
\hline
\end{tabular}

NMR Spectra and HPLC Chromatographs of $\mathbf{2 t}$

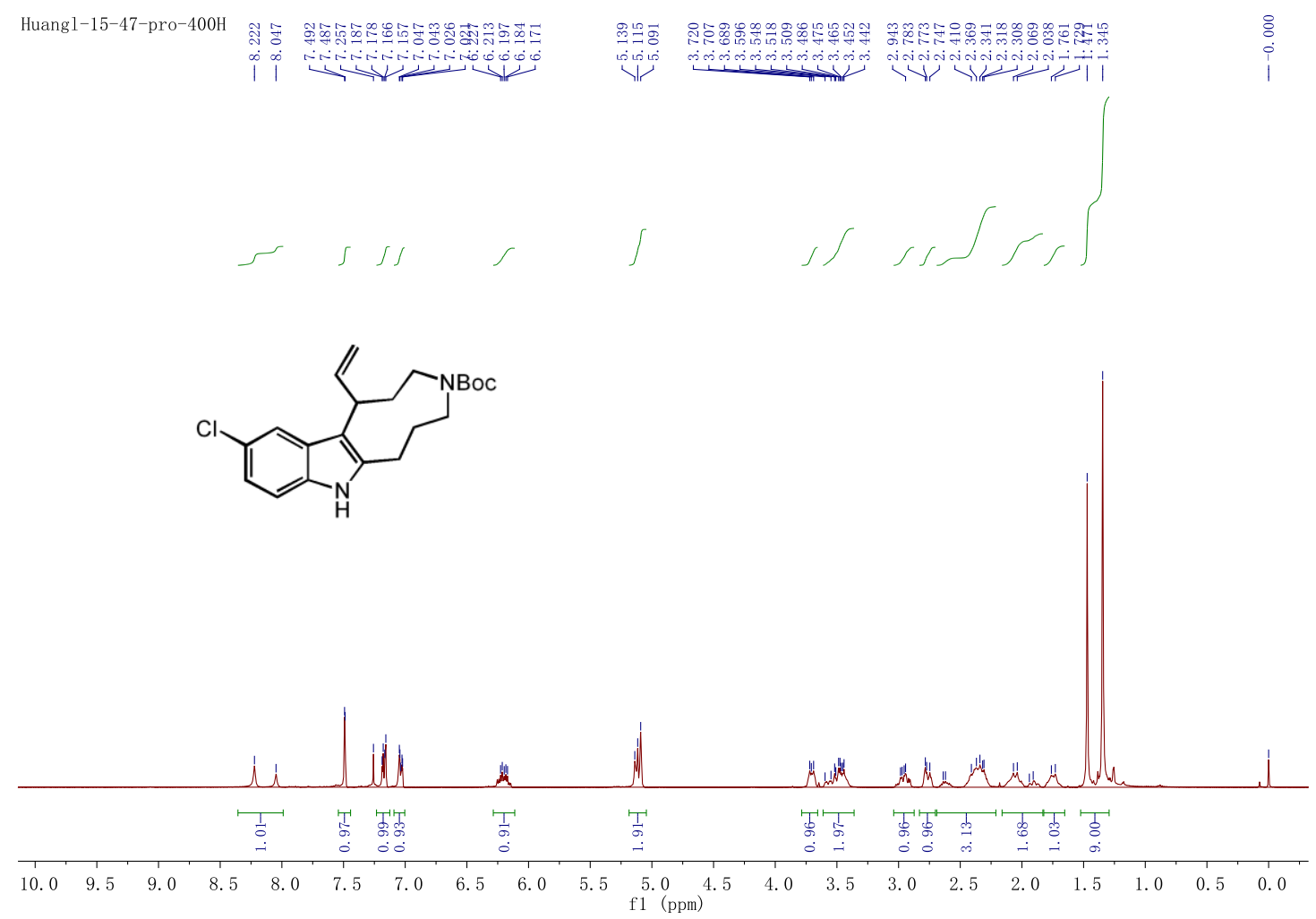




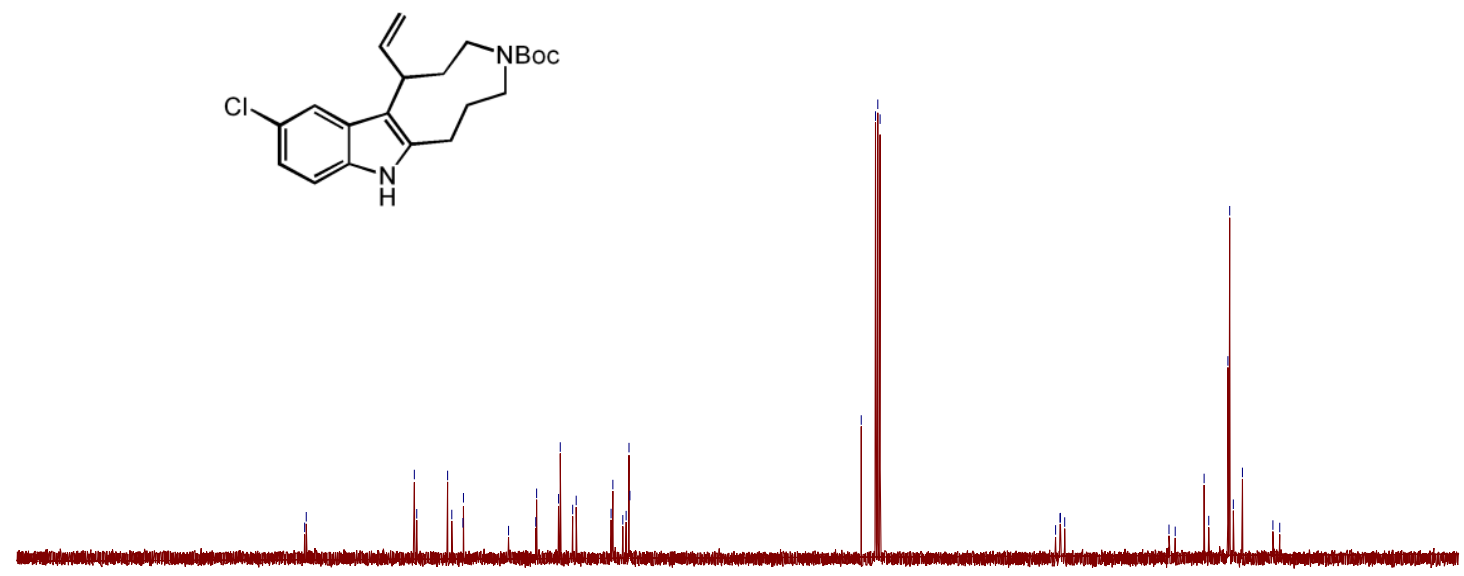

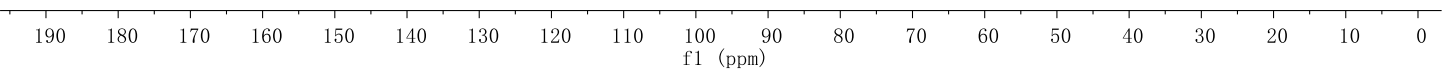

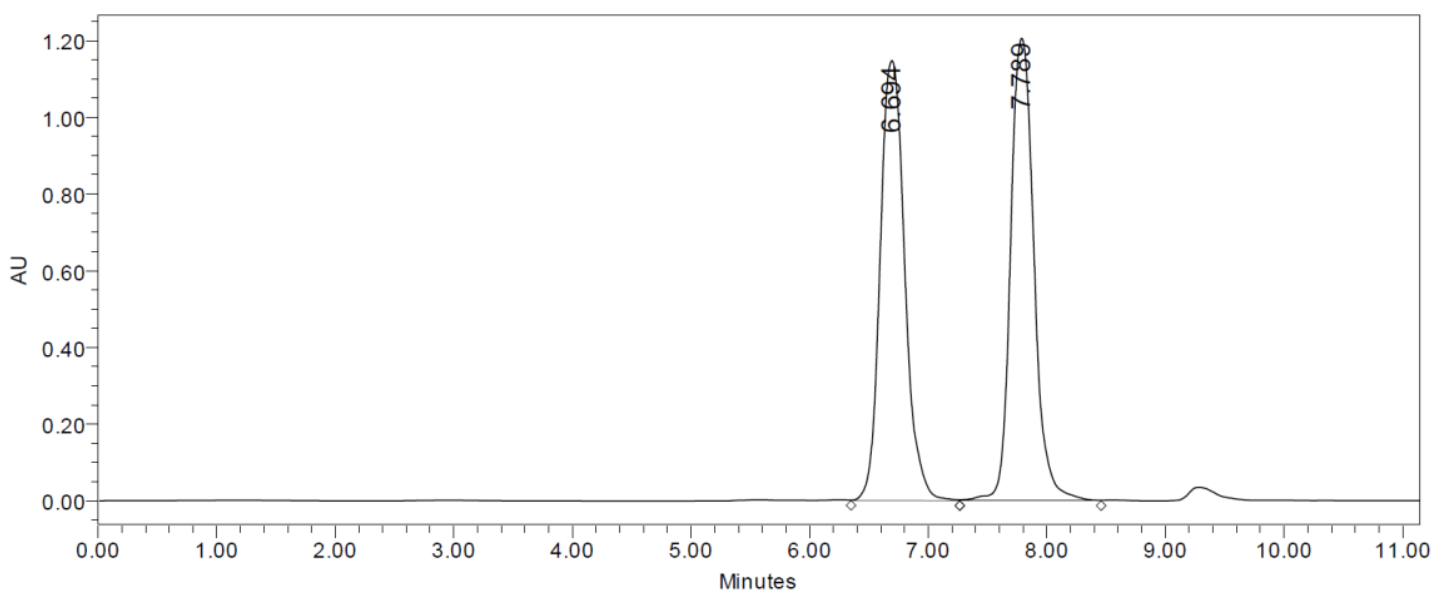

Channel: W2489 ChA; Processed Channel: W2489 ChA 230nm; Injection: 1; Date Acquired: 1/23/2016 1:22:36 PM CST; Result Id: 3069; Processing Method: fghjfh

Peak Name:

\begin{tabular}{|c|r|c|c|r|c|}
\hline & Injection & RT & Area & $\%$ Area & Height \\
\hline 1 & 1 & 7.789 & 16534146 & 50.07 & 1205834 \\
\hline 2 & 1 & 6.694 & 16488320 & 49.93 & 1146983 \\
\hline Mean & & 7.242 & & & \\
\hline Std. Dev. & & 0.774 & & & \\
\hline \% RSD & & 10.69 & & & \\
\hline
\end{tabular}




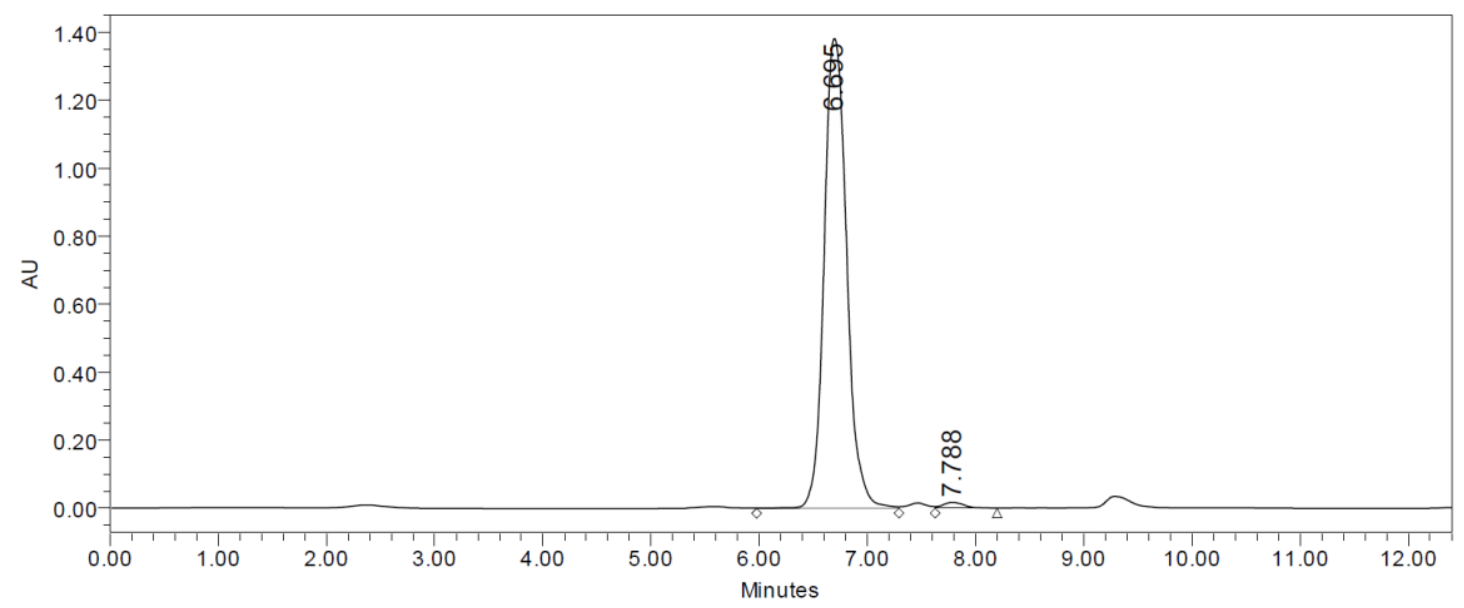

Channel: W2489 ChA; Processed Channel: W2489 ChA 230nm; Injection: 1; Date Acquired: 1/23/2016 1:34:09 PM CST; Result ld: 3071; Processing Method: fdhgs

Peak Name:

\begin{tabular}{|c|r|c|c|r|r|}
\hline & Injection & RT & Area & \% Area & Height \\
\hline 1 & 1 & 7.788 & 227012 & 1.12 & 16595 \\
\hline 2 & 1 & 6.695 & 20061053 & 98.88 & 1382075 \\
\hline Mean & & 7.241 & & & \\
\hline Std. Dev. & & 0.773 & & & \\
\hline \% RSD & & 10.67 & & & \\
\hline
\end{tabular}

NMR Spectra and HPLC Chromatographs of 3a

Huang1-6-8-pro-400H

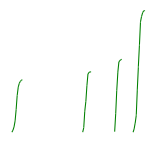<smiles>CCC1CNCCc2[nH]c3ccccc3c21</smiles>

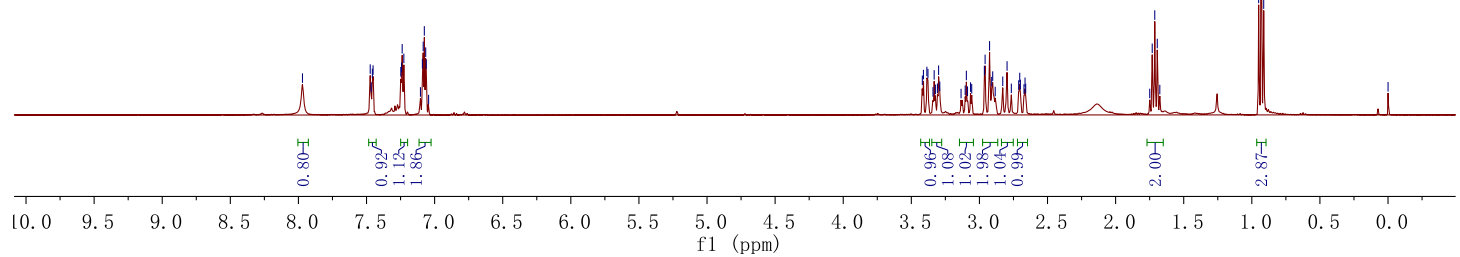


<smiles>CCC1CNCCc2[nH]c3ccccc3c21</smiles>
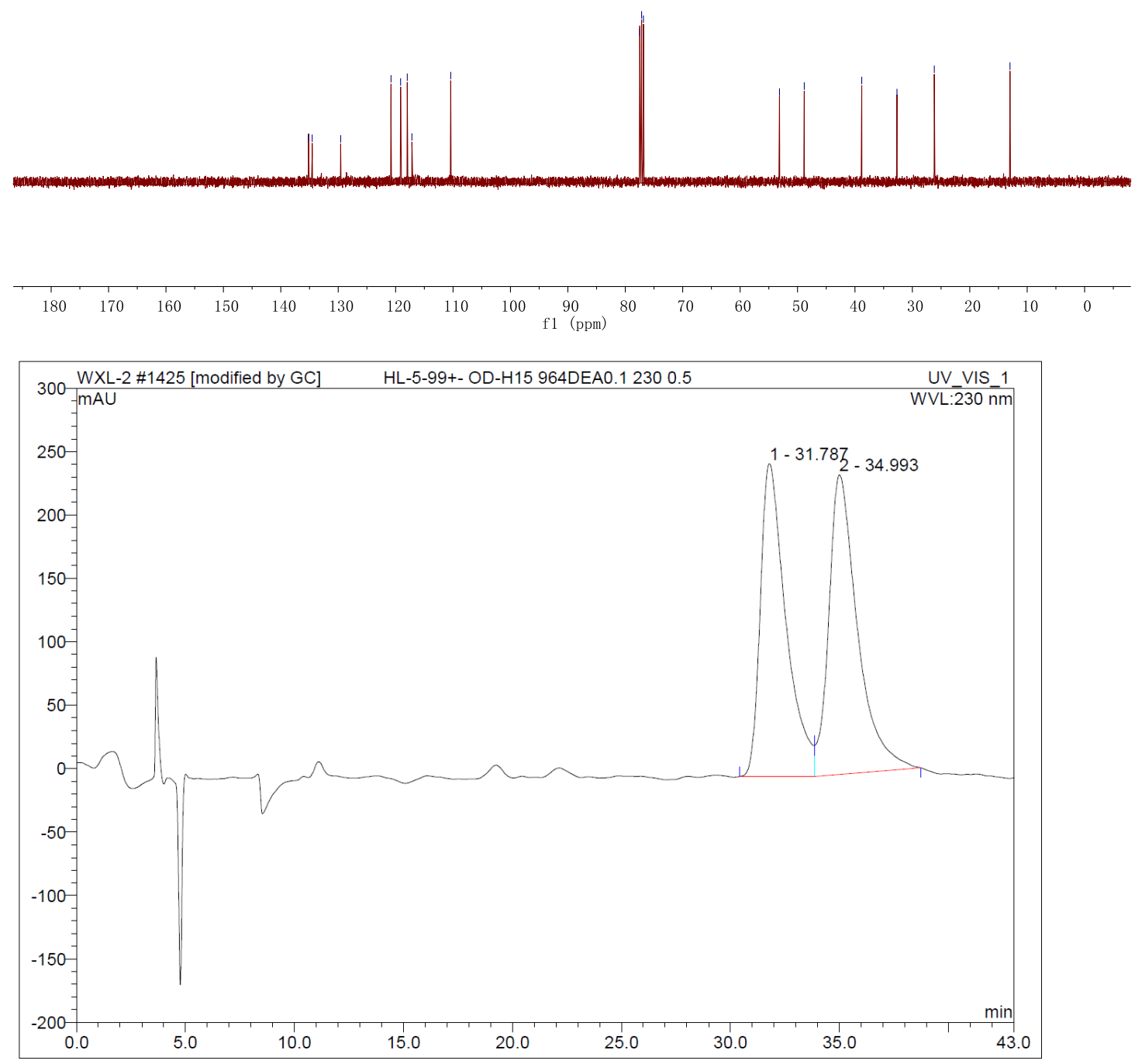

\begin{tabular}{|r|ccccccc|}
\hline No. & $\begin{array}{c}\text { Ret.Time } \\
\text { min }\end{array}$ & Peak Name & $\begin{array}{c}\text { Height } \\
\text { mAU }\end{array}$ & $\begin{array}{c}\text { Area } \\
\text { mAU*min }\end{array}$ & $\begin{array}{c}\text { Rel.Area } \\
\%\end{array}$ & Amount & Type \\
\hline 1 & 31.79 & n.a. & 246.509 & 335.055 & 48.16 & n.a. & BM $^{*}$ \\
2 & 34.99 & n.a. & 236.068 & 360.609 & 51.84 & n.a. & MB $^{*}$ \\
\hline Total: & & & 482.577 & 695.664 & 100.00 & 0.000 & \\
\hline
\end{tabular}




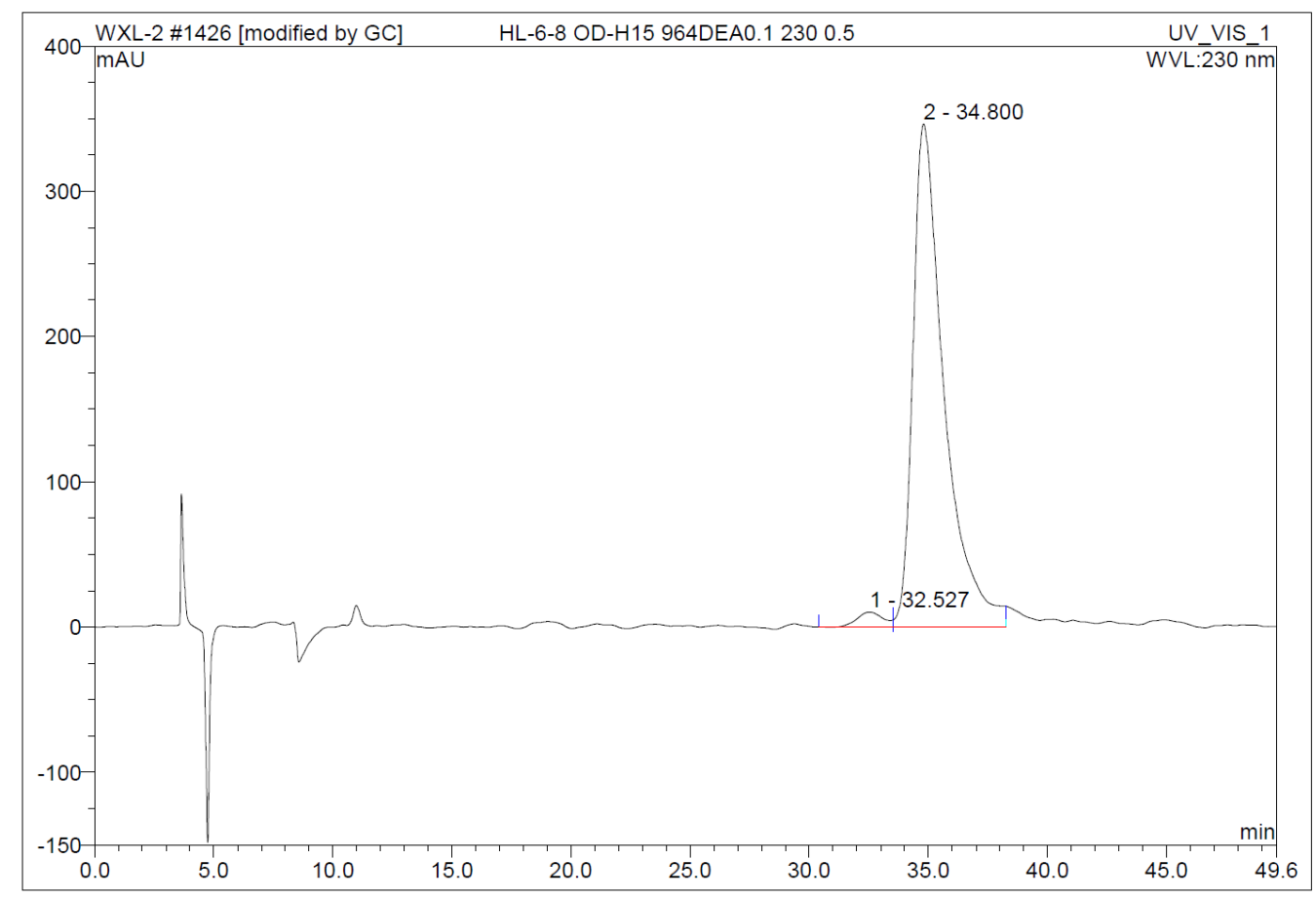

\begin{tabular}{|r|ccrrrrr|}
\hline No. & $\begin{array}{c}\text { Ret.Time } \\
\text { min }\end{array}$ & Peak Name & $\begin{array}{c}\text { Height } \\
\text { mAU }\end{array}$ & $\begin{array}{c}\text { Area } \\
\text { mAU*min }\end{array}$ & $\begin{array}{r}\text { Rel.Area } \\
\%\end{array}$ & Amount & Type \\
\hline 1 & 32.53 & n.a. & 10.237 & 13.039 & 2.42 & n.a. & BM $^{*}$ \\
2 & 34.80 & n.a. & 346.300 & 525.425 & 97.58 & n.a. & M * $^{*}$ \\
\hline Total: & & & 356.537 & 538.463 & 100.00 & 0.000 & \\
\hline
\end{tabular}

NMR Spectra and HPLC Chromatographs of $\mathbf{4 a}$

Huang1-6-23-pro-400H-DMSO Std proton

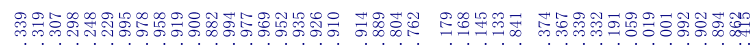

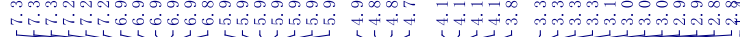
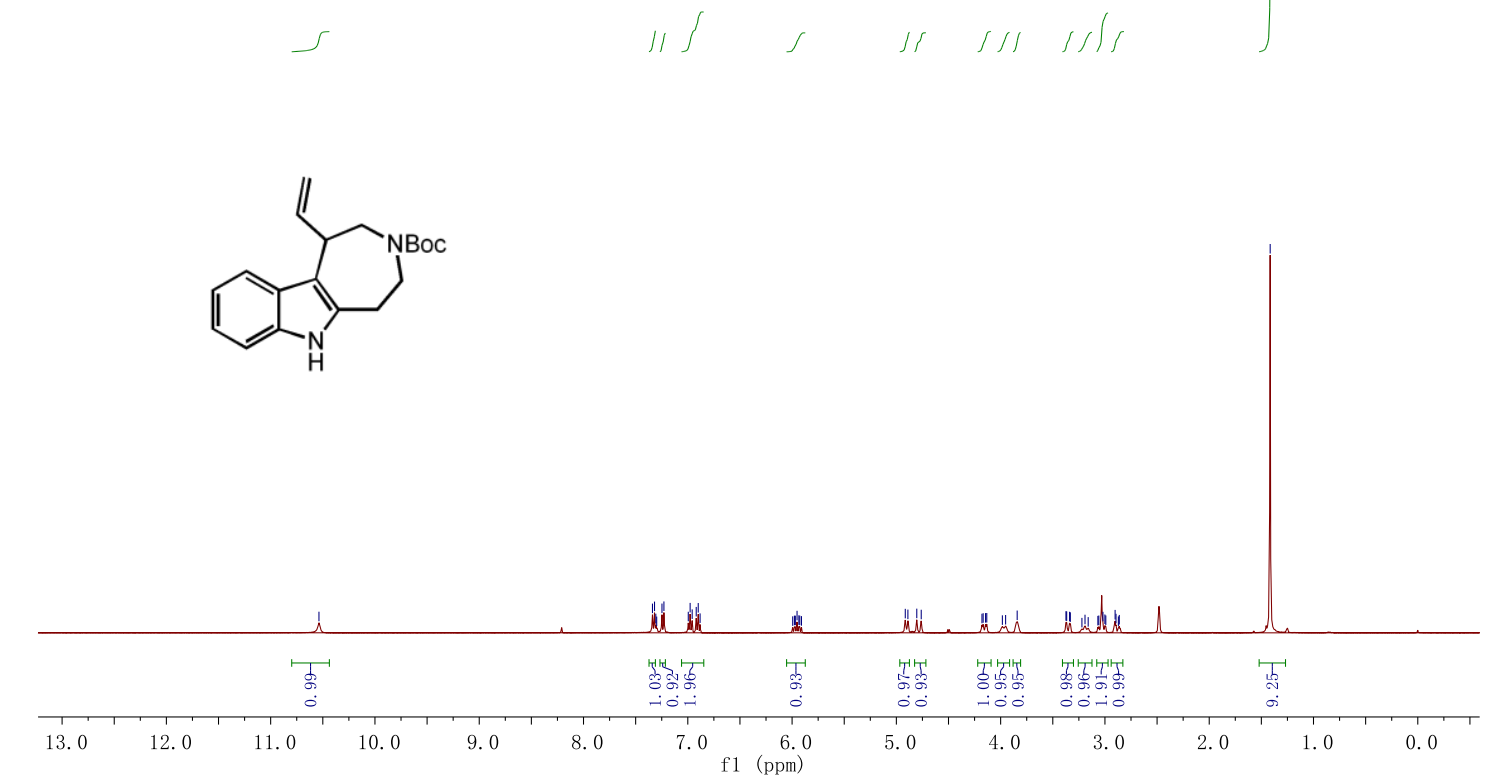
<smiles></smiles>

$\begin{array}{rrrrrrrrrrrrrrrrrrr}1 & 1 \\ 190 & 180 & 170 & 160 & 150 & 140 & 130 & 120 & 110 & \begin{array}{c}100 \\ \mathrm{f} 1\end{array}(\mathrm{ppm}) & 80 & 70 & 60 & 50 & 40 & 30 & 20 & 10 & 0\end{array}$

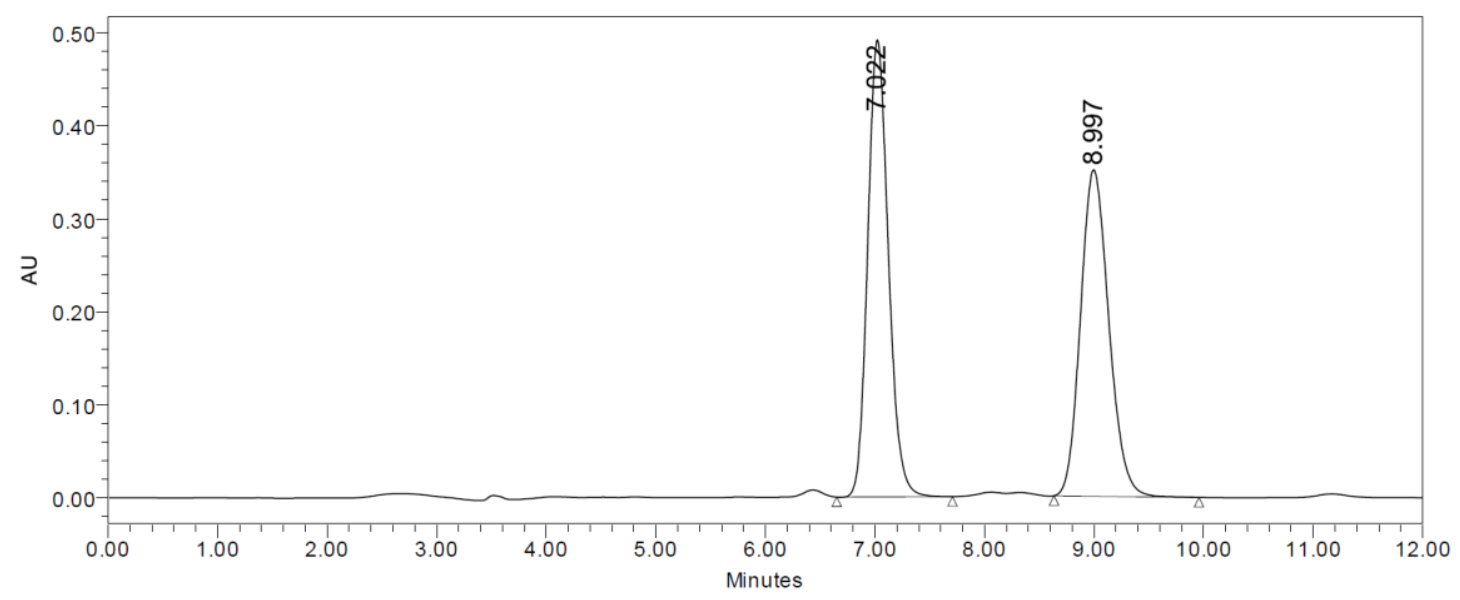

Channel: W2489 ChA; Processed Channel: W2489 ChA 230nm; Injection: 1; Date Acquired: 9/5/2014 8:56:23 AM CST; Result Id: 1434; Processing Method: 1as

\begin{tabular}{|c|r|c|c|r|c|}
\multicolumn{7}{|c}{ Peak Name: } \\
\hline & Injection & RT & Area & $\%$ Area & Height \\
\hline 1 & 1 & 8.997 & 6304275 & 49.46 & 350955 \\
\hline 2 & 1 & 7.022 & 6441872 & 50.54 & 491575 \\
\hline Mean & & 8.009 & & & \\
\hline Std. Dev. & & 1.396 & & & \\
\hline \% RSD & & 17.43 & & & \\
\hline
\end{tabular}




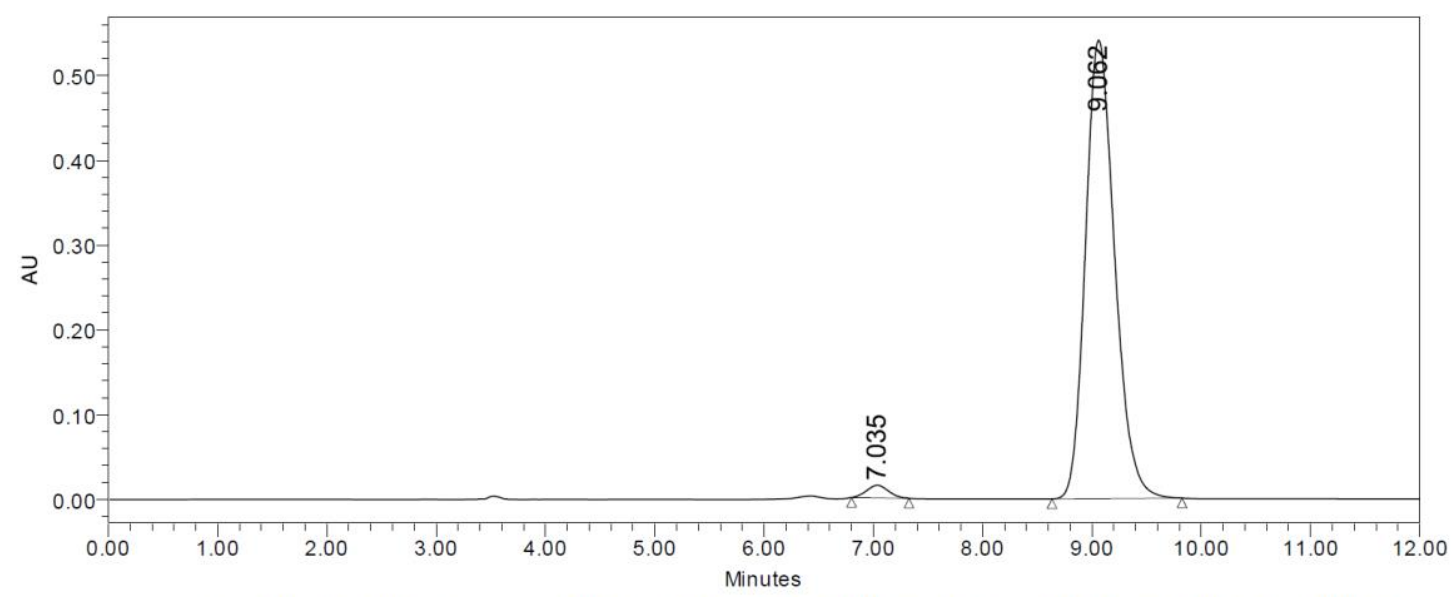

Channel: W2489 ChA; Processed Channel: W2489 ChA 230nm; Injection: 1; Date Acquired: 9/10/2014 10:30:22 PM CST; Result Id: 1448; Processing Method: qw

Peak Name:

\begin{tabular}{|c|r|c|r|r|r|}
\hline & Injection & RT & Area & \% Area & Height \\
\hline 1 & 1 & 9.062 & 10065592 & 98.00 & 540990 \\
\hline 2 & 1 & 7.035 & 205065 & 2.00 & 14811 \\
\hline Mean & & 8.048 & & & \\
\hline Std. Dev. & & 1.433 & & & \\
\hline \% RSD & & 17.80 & & & \\
\hline
\end{tabular}

NMR Spectra and HPLC Chromatographs of 5a

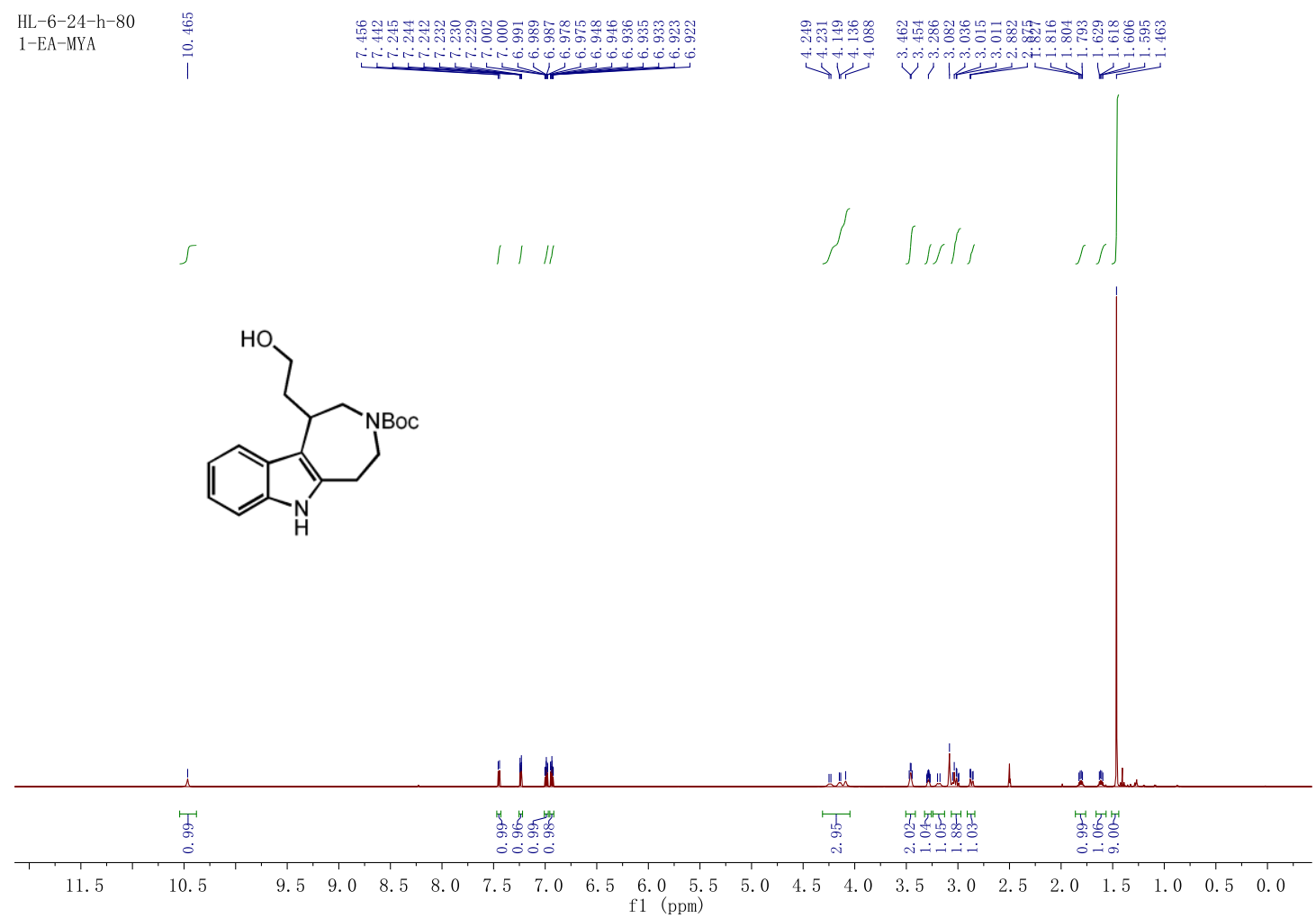



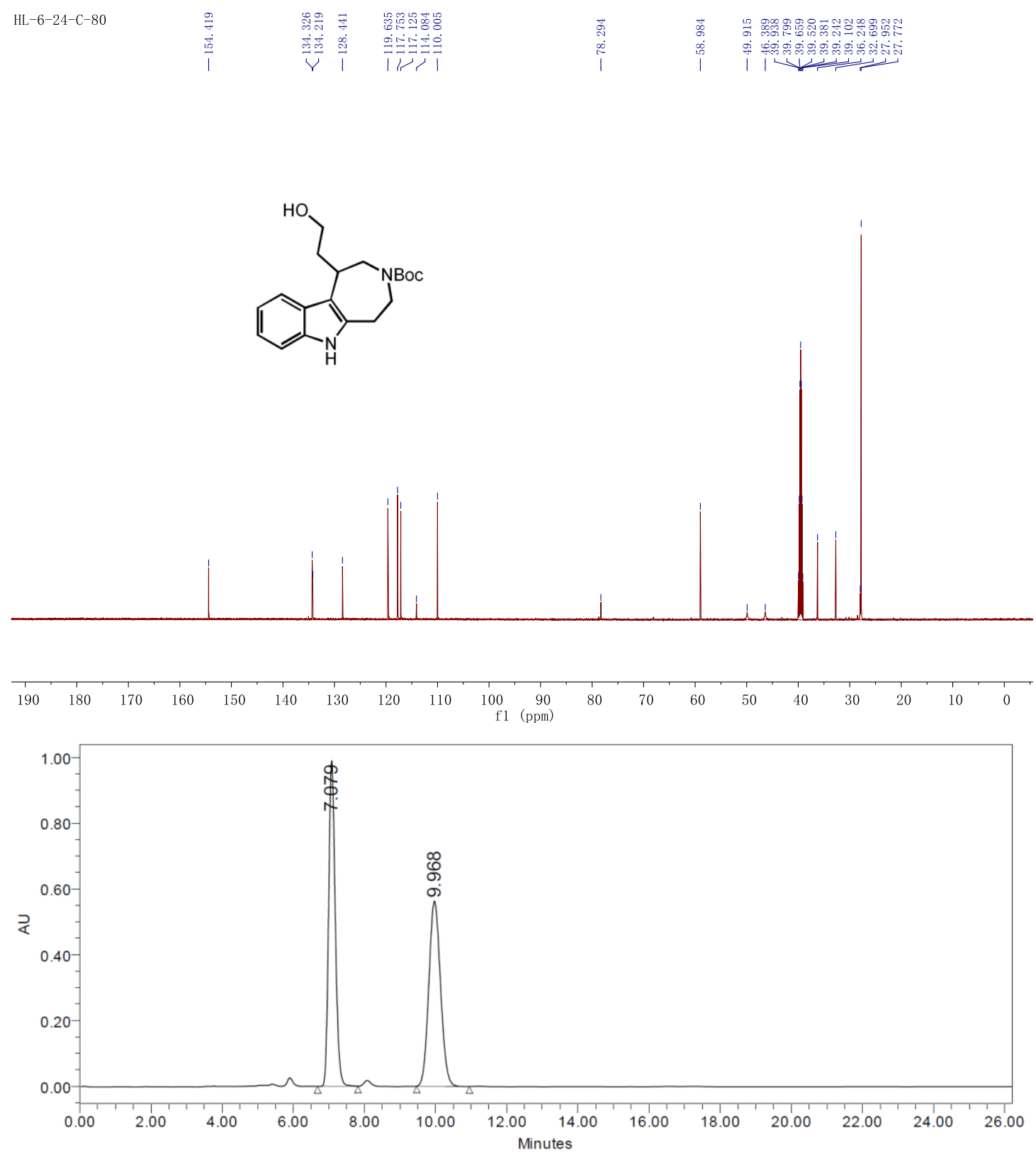

Channel: W2489 ChA; Processed Channel: W2489 ChA 230nm; Injection: 1; Date Acquired: 9/12/2014 10:41:26 PM CST; Result Id: 1506; Processing Method: qq

Peak Name:

\begin{tabular}{|c|r|c|c|r|c|}
\hline & Injection & RT & Area & \% Area & Height \\
\hline 1 & 1 & 9.968 & 12873895 & 50.36 & 562985 \\
\hline 2 & 1 & 7.079 & 12691292 & 49.64 & 990934 \\
\hline Mean & & 8.523 & & & \\
\hline Std. Dev. & & 2.043 & & & \\
\hline \% RSD & & 23.97 & & & \\
\hline
\end{tabular}




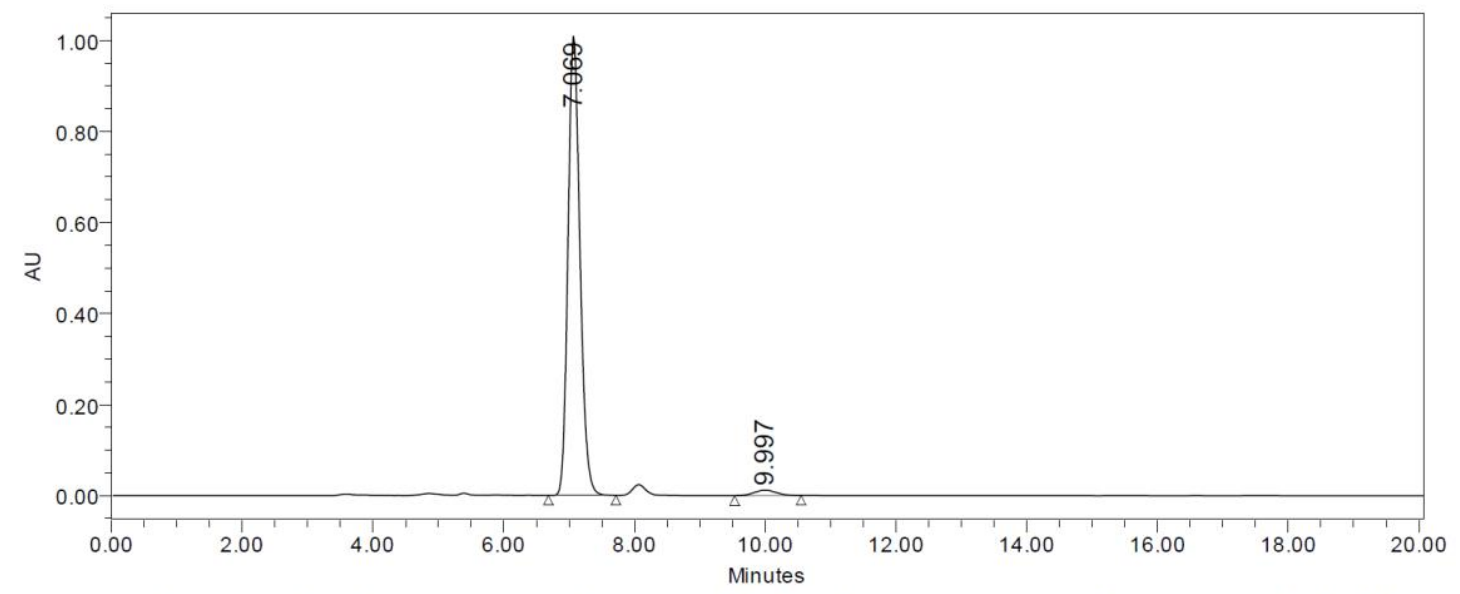

Channel: W2489 ChA; Processed Channel: W2489 ChA 230nm; Injection: 1; Date Acquired: 9/12/2014 11:08:03 PM CST; Result ld: 1509; Processing Method: ww

Peak Name:

\begin{tabular}{|c|r|c|c|r|r|}
\hline & Injection & RT & Area & $\%$ Area & Height \\
\hline 1 & 1 & 9.997 & 266902 & 2.03 & 11741 \\
\hline 2 & 1 & 7.069 & 12861199 & 97.97 & 1008877 \\
\hline Mean & & 8.533 & & & \\
\hline Std. Dev. & & 2.070 & & & \\
\hline \% RSD & & 24.26 & & & \\
\hline
\end{tabular}

NMR Spectra and HPLC Chromatographs of 7

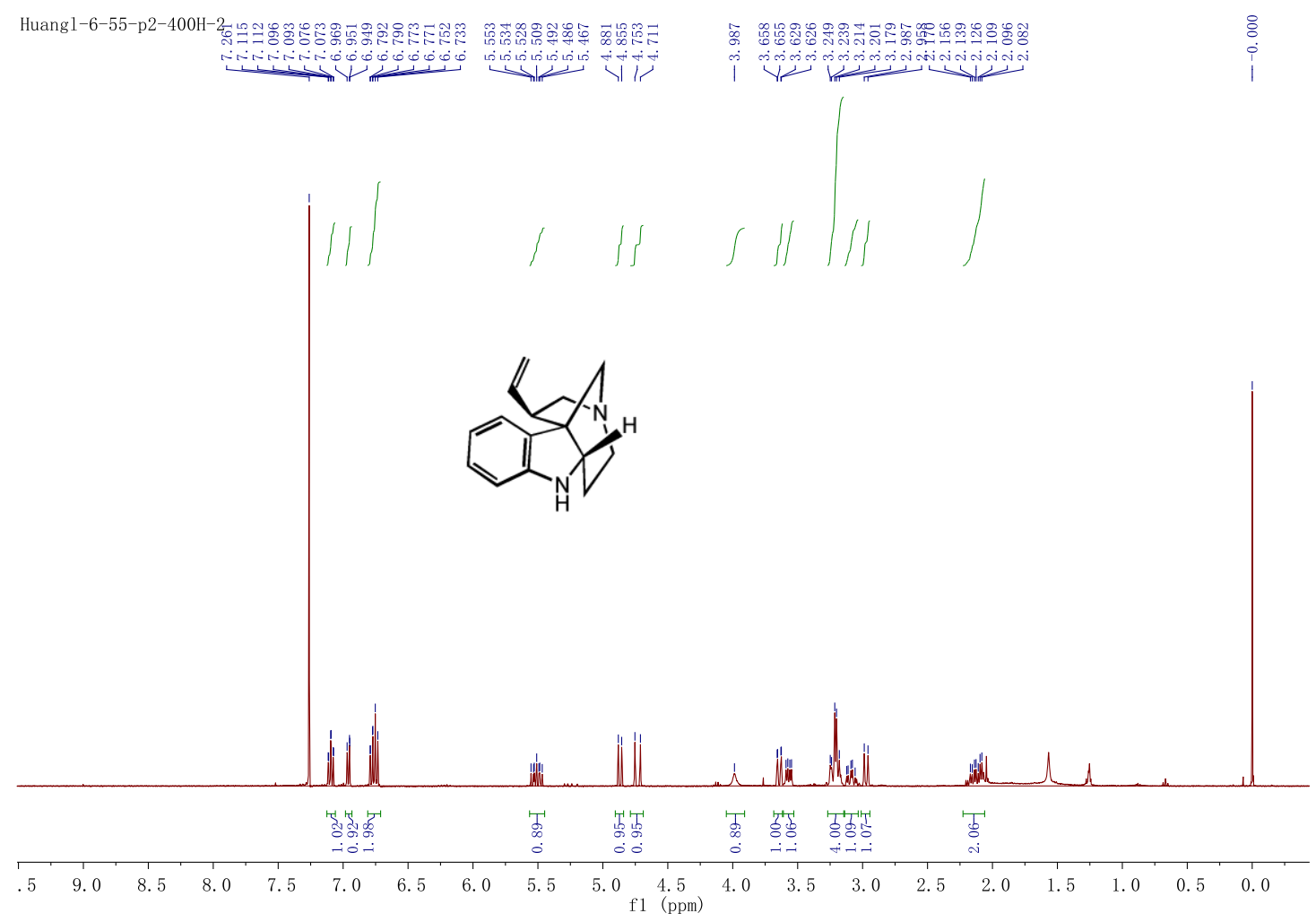



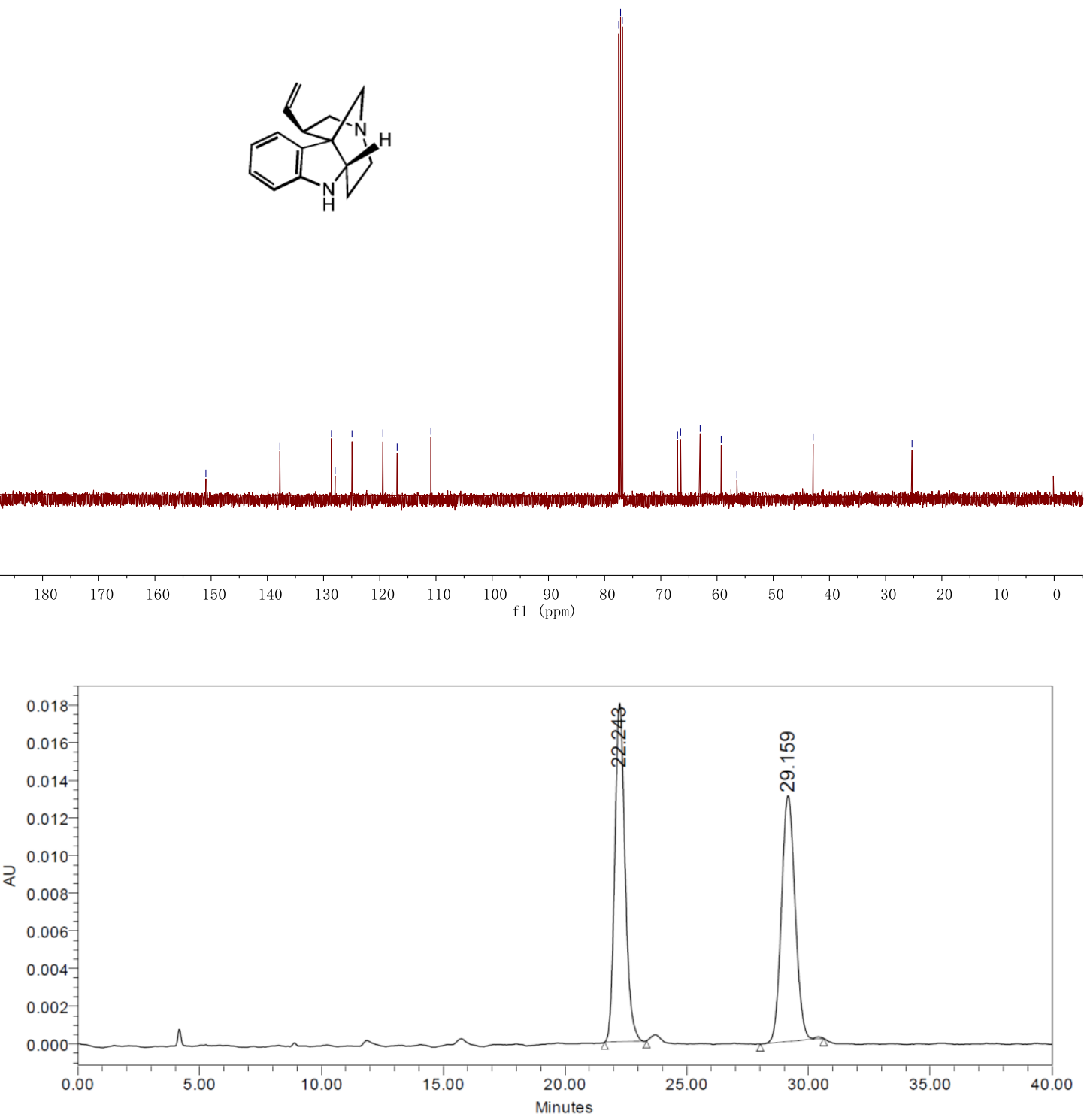

Channel: W2489 ChA; Processed Channel: W2489 ChA 254nm; Injection: 1; Date Acquired: 10/18/2014 9:58:59 AM CST; Result ld: 1572; Processing Method: 245

Peak Name:

\begin{tabular}{|c|r|r|r|r|r|}
\hline & Injection & RT & Area & $\%$ Area & Height \\
\hline 1 & 1 & 29.159 & 510222 & 49.16 & 13065 \\
\hline 2 & 1 & 22.243 & 527708 & 50.84 & 17982 \\
\hline Mean & & 25.701 & & & \\
\hline Std. Dev. & & 4.891 & & & \\
\hline \% RSD & & 19.03 & & & \\
\hline
\end{tabular}




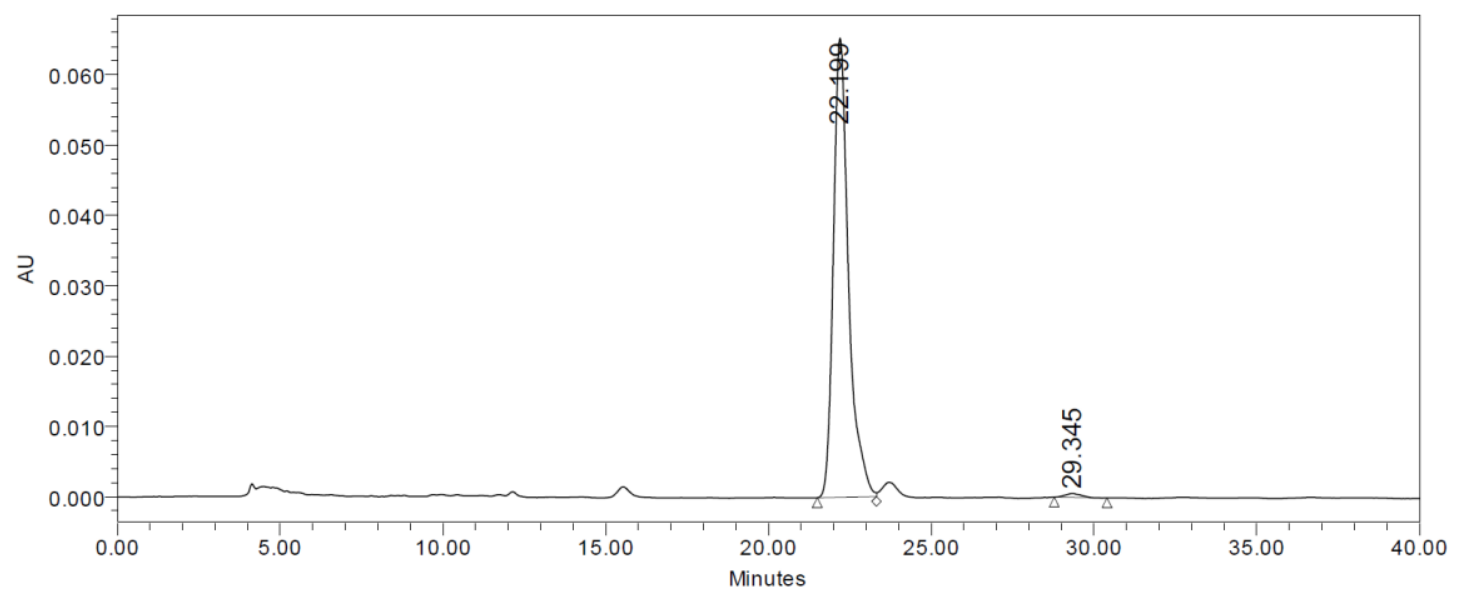

Channel: W2489 ChA; Processed Channel: W2489 ChA 254nm; Injection: 1; Date Acquired: 10/18/2014 10:39:22 AM CST; Result Id: 1575; Processing Method: 1wfesw

Peak Name:

\begin{tabular}{|c|r|r|r|r|r|}
\hline & Injection & RT & Area & \% Area & Height \\
\hline 1 & 1 & 22.199 & 2115100 & 99.11 & 65272 \\
\hline 2 & 1 & 29.345 & 18984 & 0.89 & 539 \\
\hline Mean & & 25.772 & & & \\
\hline Std. Dev. & & 5.053 & & & \\
\hline \% RSD & & 19.60 & & & \\
\hline
\end{tabular}

Copies of 2D-NMR Spectra (impure) 

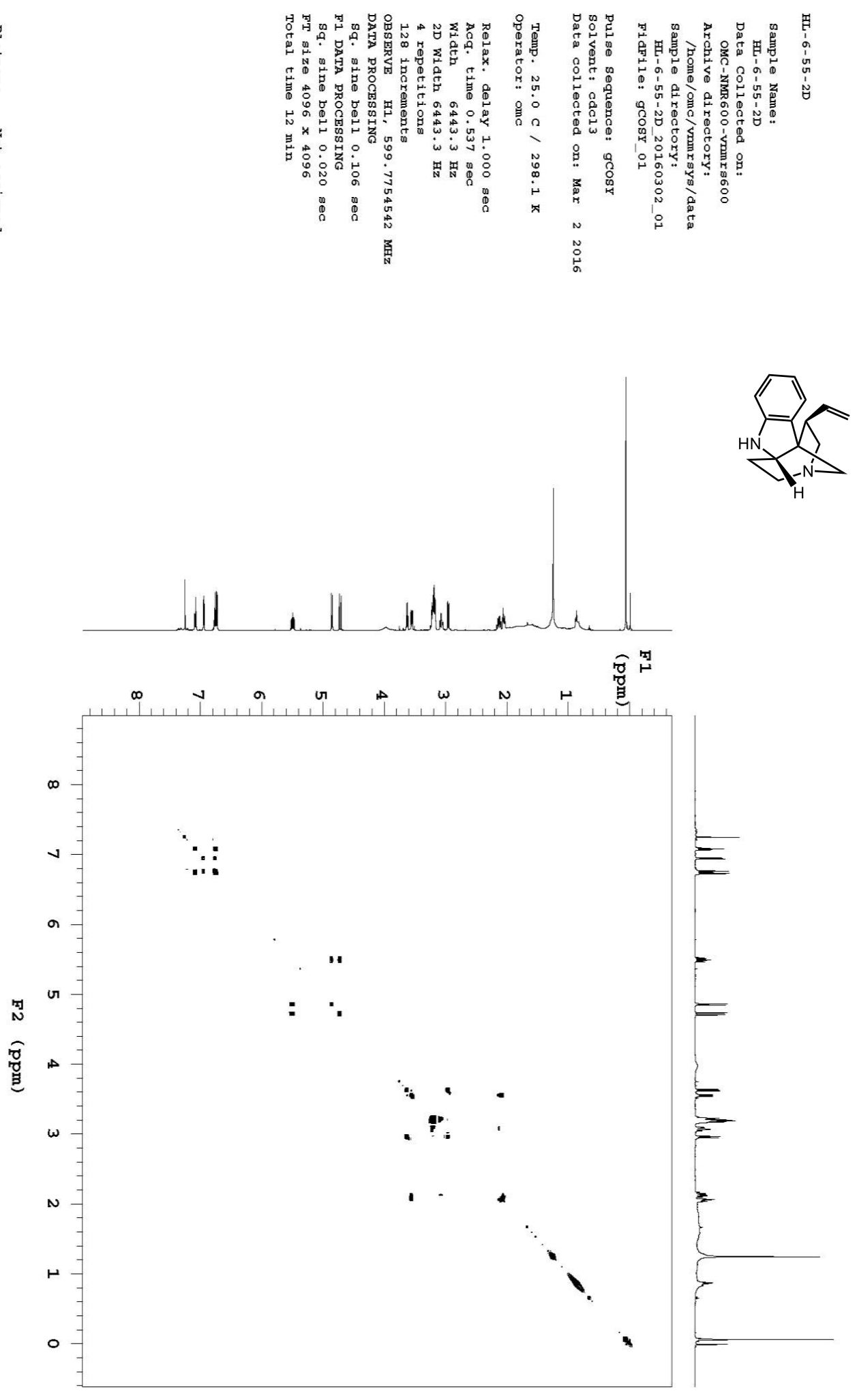

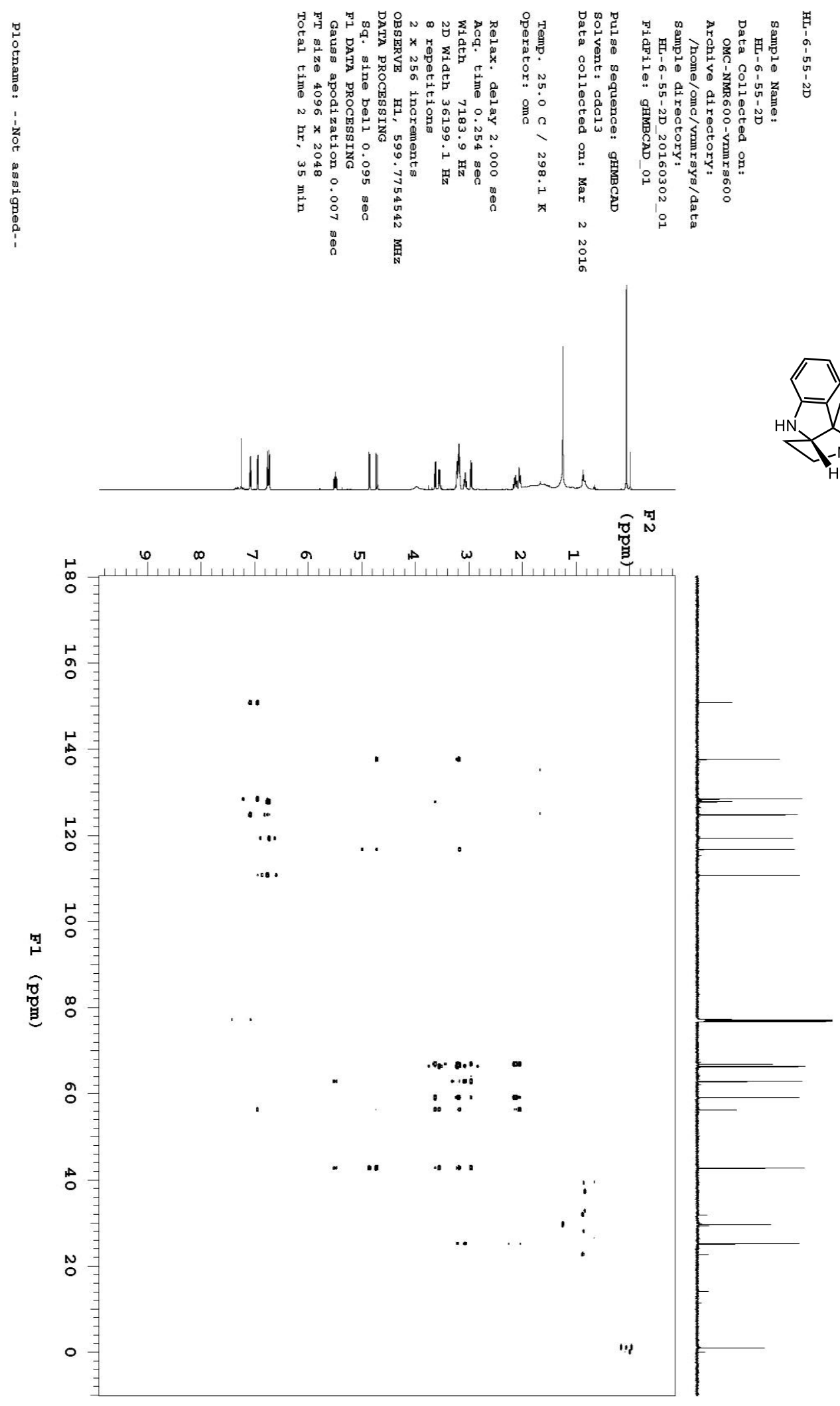


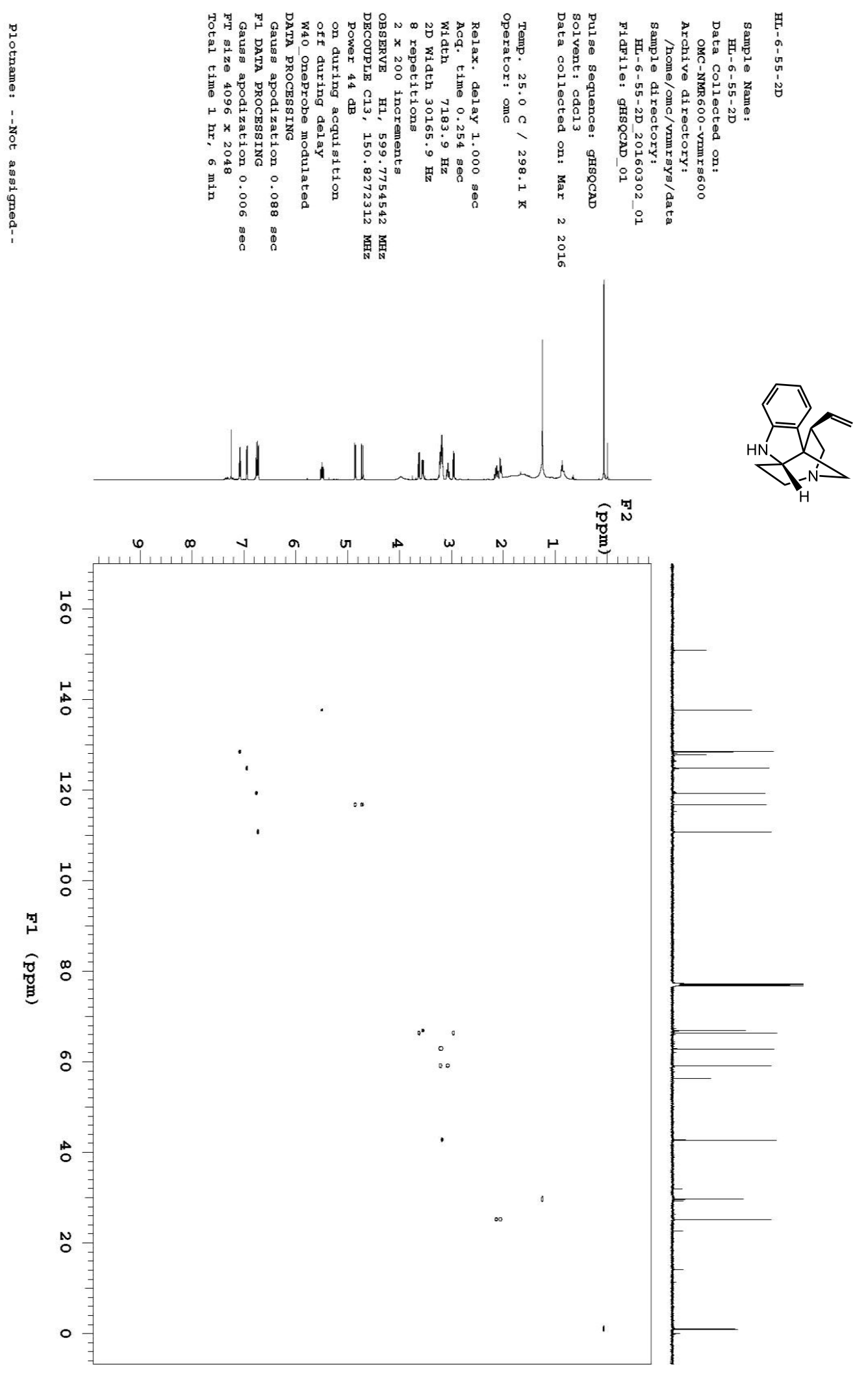



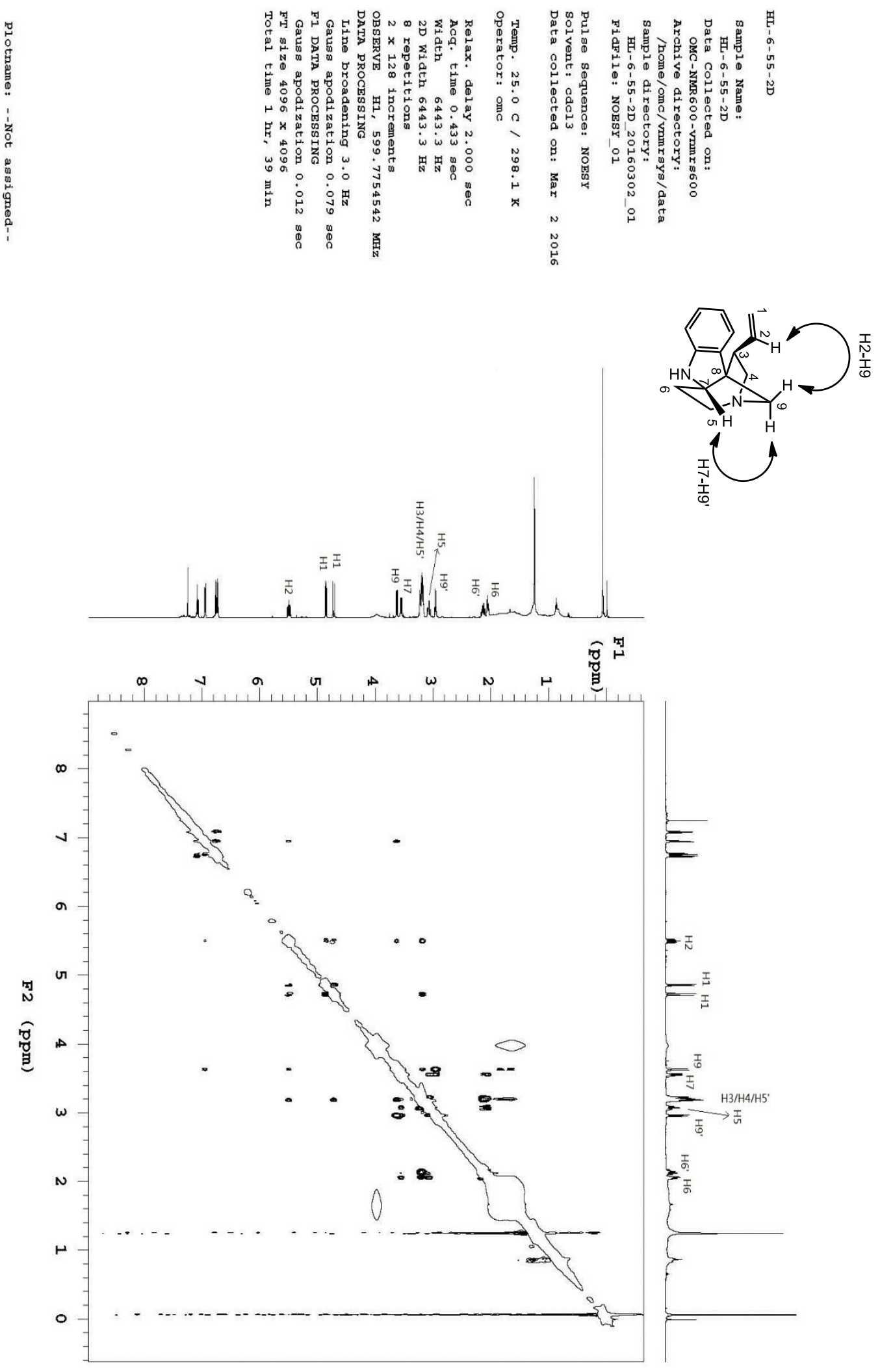

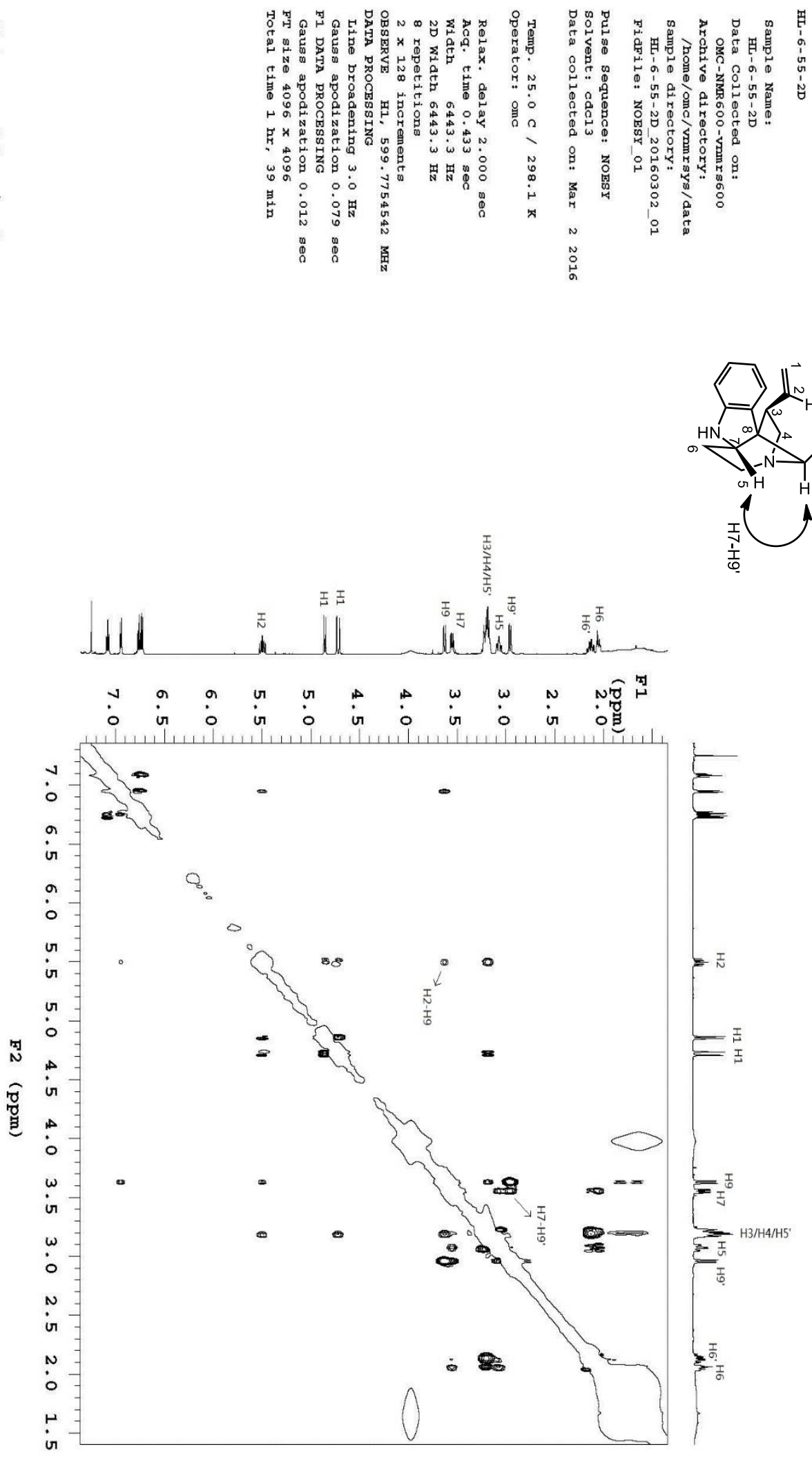